\title{
First-principles calculations of polaronic correlations and reactivity of oxides: manganites, water oxidation and $\mathrm{Pd} /$ rutile interface
}

\section{Dissertation}

zur Erlangung des mathematisch-naturwissenschaftlichen Doktorgrades

"Doctor rerum naturalium"

der Georg-August-Universität Göttingen

im Promotionsprogramm ProPhys

der Georg-August University School of Science (GAUSS)

vorgelegt von

\section{Mohsen Sotoudeh}

aus Shahreza, Iran 


\section{Betreuungsausschuss}

Prof. Dr. Peter E. Blöchl, Institut für Theoretische Physik, TU Clausthal

Prof. Dr. Jörg Behler, Institut für Physikalische Chemie

Prof. Dr. Christian Jooss, Institut für Materialphysik

\section{Mitglieder der Prüfungskommission}

Referent: Prof. Dr. Peter E. Blöchl, Institut für Theoretische Physik, TU Clausthal Korreferent: Prof. Dr. Christian Jooss, Institut für Materialphysik

\section{Weitere Mitglieder der Prüfungskommission}

Prof. Dr. Marcus Müller, Institut für Theoretische Physik

Prof. Dr. Jörg Behler, Institut für Physikalische Chemie

Prof. Dr. Vasile Moshnyaga, I. Physikalisches Institut

PD Dr. Salvatore R. Manmana, Institut für Theoretische Physik

Tag der mündlichen Prüfung: 12.12.2018 


\section{Contents}

1 Introduction $\quad 7$

2 Computational methods $\quad 11$

2.1 Born-Oppenheimer approximation . . . . . . . . . . . . . . . . 11

2.1.1 Classical approximation . . . . . . . . . . . . . . . . . 14

2.2 Variational method . . . . . . . . . . . . . . . . . . . . 15

2.3 Density-Functional Theory . . . . . . . . . . . . . . . . 16

2.3.1 Kohn-Sham equations . . . . . . . . . . . . . . . 18

2.3.2 Exchange-correlation functional . . . . . . . . . . . . . 20

2.3.3 Local density approximation (LDA) . . . . . . . . . . . . . . . . . 23

2.3.4 Generalized gradient approximation (GGA) . . . . . . . . . 23

2.3.5 Hybrid functionals . . . . . . . . . . . . . . . . . 24

2.3.6 Local hybrid functional (PBE0r) . . . . . . . . . . . . . 25

2.3.7 Benchmarks ....................... 26

2.4 Projector augmented wave method . . . . . . . . . . . . . . . 26

2.4.1 Frozen core approximation . . . . . . . . . . . . . . 28

2.4.2 Expectation values of local operators . . . . . . . . . . . . . 28

2.4 .3 Density . . . . . . . . . . . . . . . . . . . . . 28

2.5 Ab-initio Molecular dynamics . . . . . . . . . . . . . . . . . . . . 29

2.6 Calculation of spectra . . . . . . . . . . . . . . . . . . . . . . . 29

2.6.1 ELNES and XANES . . . . . . . . . . . . . . . . . . . . . . . 29

2.7 Computation codes . . . . . . . . . . . . . . . . . . . . . . . . . . . 31

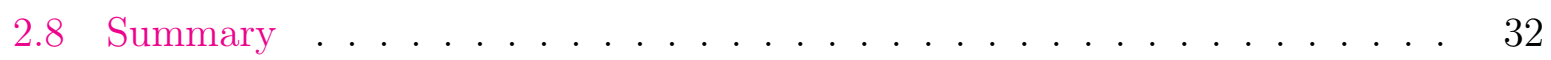

3 Manganites 33

3.1 Importance of manganites . . . . . . . . . . . . . . . . . 33 
3.2 Perovskite lattice . . . . . . . . . . . . . . . . . . . . . 34

3.2 .1 Ideal structure . . . . . . . . . . . . . . . . . . . . . . . 34

3.2 .2 Octahedral tilting . . . . . . . . . . . . . . . . . . . . . . . . . . . . . . . . . . . 35

3.3 Energy levels . . . . . . . . . . . . . . . . . . . . . . 37

3.3 .1 Crystal field splitting . . . . . . . . . . . . . . . . . 37

3.3 .2 Jahn-Teller distortion . . . . . . . . . . . . . . . . . . . . 38

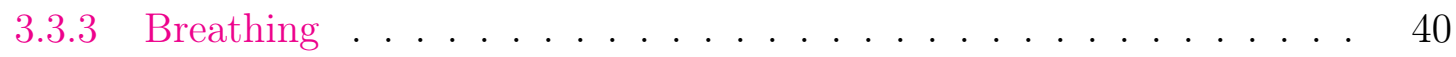

3.4 Magnetic order . . . . . . . . . . . . . . . . . . . . 40

3.5 The $\mathrm{Pr}_{1-x} \mathrm{Ca}_{x} \mathrm{MnO}_{3}$ Phase Diagram . . . . . . . . . . . . . . . . . . . . 41

3.5.1 Double exchange and the FM phase . . . . . . . . . . . . . . . 43

3.5.2 Superexchange and the AF Charge Ordered Phase . . . . . . . . . . 44

3.5.3 Discussion of Double Exchange and Superexchange . . . . . . . . . 44

3.6 Models and parameters . . . . . . . . . . . . . . . . 45

3.7 Electronic structure of $\mathrm{Pr}_{1-x} \mathrm{Ca}_{x} \mathrm{MnO}_{3} \ldots \ldots \ldots \ldots$

3.7.1 Stoichiometric $\mathrm{CaMnO}_{3} \ldots \ldots \ldots \ldots$. . . . . . . . . 46

3.7 .2 Stoichiometric $\mathrm{PrMnO}_{3} \ldots \ldots \ldots \ldots$. . . . . . . . . . 50

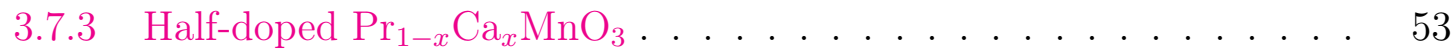

3.8 Experimental verification of the calculations . . . . . . . . . . 57

3.8.1 Role of the $U$-tensor . . . . . . . . . . . . . . . . . . 58

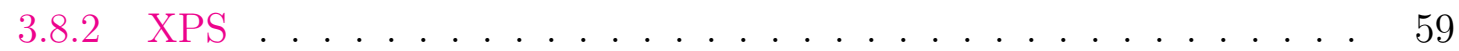

3.8 .3 ELNES and XANES . . . . . . . . . . . . . . . 60

$3.9 \mathrm{Pr}_{1-x} \mathrm{Ca}_{x} \mathrm{MnO}_{3}$ overview . . . . . . . . . . . . . . . . . . 63

3.10 Electronic structure of $\mathrm{La}_{1-x} \mathrm{Sr}_{x} \mathrm{MnO}_{3} \quad \ldots \ldots \ldots$

3.10 .1 Stoichiometric $\mathrm{SrMnO}_{3} \ldots \ldots \ldots$. . . . . . . . . . 65

3.10 .2 Stoichiometric $\mathrm{LaMnO}_{3}$. . . . . . . . . . . . . . . . . . 67

3.10.3 Stoichiometric $\mathrm{La}_{0.66} \mathrm{Sr}_{0.33} \mathrm{MnO}_{3} \ldots \ldots \ldots$. . . . . . . . . . . . . . 68

$3.10 .4 \mathrm{La}_{1-x} \mathrm{Sr}_{x} \mathrm{MnO}_{3}$ Overview . . . . . . . . . . . . . . . . . . 68

3.11 Summary . . . . . . . . . . . . . . . . . . . . . . . . . 69

4 Water splitting $\quad 71$

4.1 Catalysis for sustainable energy . . . . . . . . . . . . . . . . 71

4.2 Computational details . . . . . . . . . . . . . . . . . . 72

4.3 Water dimer radical cation . . . . . . . . . . . . . . . . . 73

4.3.1 Molecular orbitals for water molecule . . . . . . . . . . . . . . . 73

4.3.2 Plausible structure for water dimer . . . . . . . . . . . . . . 74

4.3.3 Adding a hole: proton transfer and hemibonded structure . . . . . . 75

4.3.4 Adding second hole: proton transfer vs hemibonded structure . . . 79

4.3.5 Transition state for water dimer radical cation . . . . . . . . . . . 79 
4.3.6 Potential energy surfaces of $\left(\mathrm{H}_{2} \mathrm{O}\right)_{2}^{+} \ldots \ldots \ldots \ldots$. . . . . . . . . . . . . . . . . . . . 82

4.4 Surface calculations . . . . . . . . . . . . . . . . . . . . . . 84

4.4 Computational details . . . . . . . . . . . . . . . . 85

4.4.2 Surface relaxation and reconstruction . . . . . . . . . . . . . 86

4.5 Oxygen evolution reaction . . . . . . . . . . . . . . . 86

4.5.1 The initial situation: water-covered $\mathrm{CaMnO}_{3}$ surface . . . . . . . 87

4.5.2 First Proton-Electron Transfer . . . . . . . . . . . . . . . . . . 89

4.5.3 Second Proton-Electron Transfer . . . . . . . . . . . . . . 93

4.5.4 Third Proton-Electron Transfer . . . . . . . . . . . . . . . 95

4.5.5 Fourth Proton-Electron Transfer . . . . . . . . . . . . . . . . 96

4.5.6 Calculation of the OER energy profile . . . . . . . . . . . . . 101

4.6 Summary . . . . . . . . . . . . . . . . . . . . . 102

5 Role of defects in titanium dioxide $\quad 103$

5.1 Importance of $\mathrm{TiO}_{2} \ldots \ldots \ldots \ldots$. . . . . . . . . . . . . 103

5.2 Rutile titanium dioxide . . . . . . . . . . . . . . . . . . . . . . . . . . . . . . . . . . . . . .

5.2 .1 Shape of the Ti $L$ edge . . . . . . . . . . . . . . . . . . . . 105

5.2 .2 Octahedral symmetry . . . . . . . . . . . . . . . . . . . . . . . . . . . . . . . . . 106

5.2 .3 Electronic structure of rutile . . . . . . . . . . . . . . . 107

5.3 EELS experiments on $\mathrm{Pd} / \mathrm{TiO}_{2}$ upon $\mathrm{H}$-exposure . . . . . . . . . . . 108

5.4 Simulations . . . . . . . . . . . . . . . . . . . 110

5.4 Triangle symmetry . . . . . . . . . . . . . . . . 110

5.4 .2 Method ............................ 111

5.4 .3 Interstitial hydrogen . . . . . . . . . . . . . . . . . . . 112

5.4 .4 Oxygen vacancy . . . . . . . . . . . . . . . . 116

5.4.5 Hydrogen oxygen-vacancy complex . . . . . . . . . . . . . . . 119

5.5 Corrosion . . . . . . . . . . . . . . . . . . . . 122

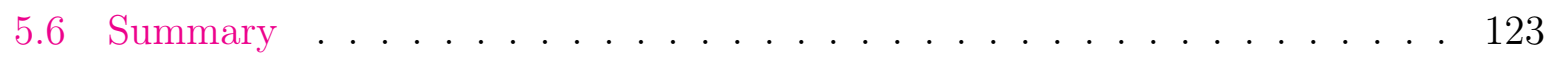

6 Discussion $\quad 125$

7 Summary $\quad 129$

$\begin{array}{ll}\text { A DFT } & 131\end{array}$

A.1 Derivation and formalism . . . . . . . . . . . . . . . . . 131

$\begin{array}{ll}\text { B The optimized xyz coordinates } & 137\end{array}$

B.1 Water-covered $\mathrm{CaMnO}_{3}(001)$ surface . . . . . . . . . . . . . . . 137

B.2 First Proton-Electron Transfer . . . . . . . . . . . . . . . . . . . . 139 
B.3 Second Proton-Electron Transfer . . . . . . . . . . . . . . . . . . . 141

B.4 Third Proton-Electron Transfer . . . . . . . . . . . . . . . . . . . . 143

B.5 Fourth Proton-Electron Transfer . . . . . . . . . . . . . . . . . . . 145

C Variable energy positron annihilation spectroscopy (VEPAS) 147

D Water formation by a hydrogenated oxygen vacancy 149

D.0.1 Introduction . . . . . . . . . . . . . . . . . . . . . . . . . 149

D.0.2 Limit of the formation enthalpy for water from rutile titanium dioxide150

D.0.3 Auxiliary calculations . . . . . . . . . . . . . . . . . . . . 152 
canere 1

\section{Introduction}

Colossal magnetoresistance (CMR) in mixed-valence compounds has attracted numerous interest in materials with perovskite structure [1,2,3], which is focused on the rich phase diagram and the magnetotransport properties [4, 5, 6]. CMR phenomena have been investigated for the manganite compounds with $\mathrm{RE}_{1-x} \mathrm{AE}_{x} \mathrm{MnO}_{3}$ general formula ( $\mathrm{RE}$ and $\mathrm{AE}$ are rare- and alkaline-earth cations). Some properties of the perovskite-type oxides depend on the doping concentration as well as growth conditions such as temperature, pressure and magnetic field (Fig. 1.1). In these materials, charge, spin, orbital and lattice degrees of freedom lead to phase transitions and the emergence of exotic phases including the pseudogap state and even phase separation $[7,8]$. They be used to design more efficient new correlated electron devices [9, 10], e.g., in magnetoelectronics and electronic memories element with smaller size.

Recently, doped manganites get more attention because of their exotic photovoltaic properties [11, 12], as well as their potential benefit as electrocatalysts [13]. Understanding and controlling of the four step reaction of water splitting at different electrocatalysts is essential for the design a highly active catalysts which is inspired by the use of a $\mathrm{CaMn}_{4} \mathrm{O}_{5}$ complex as oxygen-evolution center in natural photosynthesis [14]. For the active catalysts, different oxidation states of Mn center and the covalency type of Mn oxygen bond require to facilitate electron transfer for catalytic oxygen-evolution activity in manganite perovskites $[15,16]$. A more appropriate descriptor for catalysis is the filling of a rigid antibonding states between $\mathrm{Mn}-e_{g}$ and O- $2 p$ orbitals since it has a stronger overlap with the oxygen [17]. This means that electron transfer between surface cation and adsorbed reaction is facilitated. However, more theoretical and experimental work are needed to fully understand the oxygen evolution reaction and some descriptors do not cover all observed trends [18].

The standard density functional methods for the perovskite oxides [19] indicated that conventional functionals do not capture the electronic structure in their electron spectrum. This finding can be extended to other transition-metal oxides. To avoid the deficiencies of conventional density functional methods for transition metal compounds, $\mathrm{LDA}+U$ 


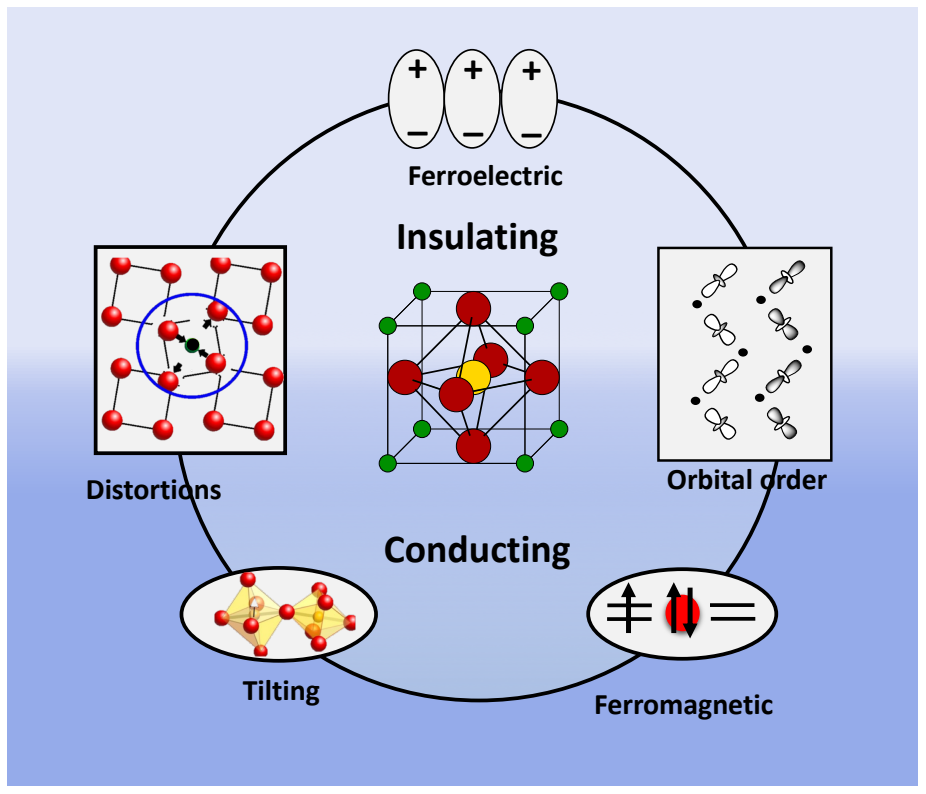

Fig. 1.1: Rich physical properties of manganites: from insulating to conducting and colossal magnetoresistance.

method [20], and hybrid density functionals [21, 22] were applied. The LDA $+U$ adds an explicit Fock term and thus introduces the atomic physics of strongly interacting electrons, while the hybrid functional have a similarity with many-particle Green's function methods such as the GW method [23, 24]. These functionals open up the band gaps and provide an improved description of spectral properties.

The most-studied compound of manganite is $\mathrm{LaMnO}_{3}$ which has an electron-rich $\mathrm{Mn}^{3+}$ character. This compound is an insulator with a band gap slightly more than $1 \mathrm{eV}$ which has orbital ordered Jahn-Teller arrangment [25, 26]. A series of analysis have been done by Ederer, Kovacic and collaborators [24, 25, 26, 27, 28, 29, 30, 31]. They performed a hybrid functionals calculations on the series of manganites which have the same electron character for Mn, by exchanging the A-type cation. They also extracted some parameters which is related to the model Hamiltonians. Although there are some theoretical approaches for these systems, but a comprehensive comparison of the valence and conduction bands, optical excitation, and structural properties with experimental data is still missing.

It is even more important for a whole doping range of manganites. The doping affects magnetization, structure, and correlations on the electronic structure. In addition, doping is the main control parameter within the complex phase diagram of manganites. Other parameters such as ion sizes [32, 8], dimensionality, external fields and temperature allow to tune the phase boundaries. However, the origin of the cooperative Jahn-Teller distortion and orbital order for $\mathrm{LaMnO}_{3}$, which is crucial for the manganites, have been under debate. 
To clarify the situation, the Kugel-Khomski [33] mechanism and the Jahn-Teller effect have been explored by using the local density approximation plus dynamical mean-field method $[34,35]$. They show that electron-phonon coupling is essential to have a local JahnTeller distortions. Thus, this supports importance of the Jahn-Teller effect in undoped manganites.

The series of $\mathrm{Pr}_{1-x} \mathrm{Ca}_{x} \mathrm{MnO}_{3}$ is of important manganite compound because of the small tolerance factor. This means that Pr and Ca anions have the similar ion sizes. This caused that there is no stable ferromagnetic metallic phase at low temperature for this compound. similarities of ion sizes, allow to change the charge of the Mn ion over the entire range from $\mathrm{Mn}^{3+}$ to $\mathrm{Mn}^{4+}$ without greatly affecting the crystal structure [36, 5].

Anisimov et al. [37], performed LDA $+U$ calculations of $\operatorname{PrMnO}_{3}$ and $\operatorname{Pr}_{1 / 2} \mathrm{Ca}_{1 / 2} \mathrm{MnO}_{3}$. They have confirmed that orbital order can be obtained from Coulomb exchange even without lattice relaxation. Van den Brink and Khomski [38], also explained the role of charge and orbital order in half-doped manganites. In the half-doped manganites, they showed formation of magnetic zig-zag chains as a result of competing magnetic and kinetic energy.

The $x=0.5$ arrangement is the so-called CE-phase, which its nature of the chargeordered state was under controversial. Experimental analysis [39] confirmed that there is no strong charge disproportionation between different Mn sites. The presence of multiferroic states with electric polarization [5], and also the formation of Mn-O-Mn dimers [40, 41] have been reported. Different theoretical studies [42, 43, 44] supported the appearance of an underlying Zener polaron type of charge ordering. The electric polarization was confirmed for half-doped materials $\operatorname{Pr}_{1 / 2} \mathrm{Ca}_{1 / 2} \mathrm{MnO}_{3}$ and $\mathrm{La}_{1 / 2} \mathrm{Ca}_{1 / 2} \mathrm{MnO}_{3}$ using the measured atomic structure by Colizzi and Giovanetti [43, 44]. Efremov et al. [42] studied the transition of the CE-type CO structure to the ordering of Zener polarons by an extended double-exchange Hamiltonian. Finding a proper description of the band structure close to the Fermi level, excitation spectrum and the type of order is a crucial step to catch a full understanding of quasiparticle excitations in the different phases.

In this work we are going to investigated the effect of doping on the structure of the valence and conduction band, magnetic and charge ordering as well as on the resulting excitation spectra for the series $\operatorname{Pr}_{1-x} \mathrm{Ca}_{x} \mathrm{MnO}_{3}$ and $\mathrm{La}_{1-x} \mathrm{Sr}_{x} \mathrm{MnO}_{3}$. We use local hybrid functionals to account for the Coulomb interaction on the Mn-sites and to obtain the physically correct description of the electronic structure. The Fock term is limited to onsite terms in a carefully crafted local orbital basisset. This makes the computational cost of hybrid calculations comparable to that of regular GGA calculations. This free parameter is determined by experimental x-ray photoelectron spectroscopy (XPS). Emphasis is given to a realistic description of the electronic structure, which is verified by comparison with electron energy-loss near-edge structure (ELNES) and the x-ray absorption near-edge structure (XANES) [45], as well as measurements of optical absorption [46]. In addition, the insight on the electronic structure is used to explain the experimental optical conductivity data. We also attempt to rationalize the high activities of $\operatorname{Pr}_{1-x} \mathrm{Ca}_{x} \mathrm{MnO}_{3}$ catalyst surface in contact with water through the electronic structure and show DFT descriptors of oxygen 
evolution reaction (OER) toward the development of novel water splitting catalysts.

Moreover, to gain insight into the corrosion reactions during the oxygen evolution reaction we investigated the defect chemistry in rutile. Rutile is considerably simpler than the manganites and allows us to make direct contact with experimental data. As shown there, the defect concentration in rutile next to a buried interface with $\mathrm{Pd}$ interface can be controlled by loading it with hydrogen. In order to rationalize the observations, we performed calculations of the oxygen vacancy, hydrogen and the hydrogen complex with the oxygen. For these calculations, the correct opening of the bandgap is crucial. In regular DFT calculations only the most positive charge state is stable, while the charging levels shift from the conduction band into the band gap when hybrid functionals are used. This effect is essential for the description of the band bending on hydrogen loading. We find that oxygen vacancy defect has charging levels in a narrow energy window, which is the expected Fermi-level pinning range. Neutral defects are only produced when oxygen vacancies exist and negative defects are not produced in the whole range of Fermi-level.

The thesis is organized as follows. Chapter 2 introduces Density Functional Theory, and some special topics on methodologies. Chapter 3 is devoted to the study of series of manganites, $\mathrm{Pr}_{1-x} \mathrm{Ca}_{x} \mathrm{MnO}_{3}$ and $\mathrm{La}_{1-x} \mathrm{Sr}_{x} \mathrm{MnO}_{3}$ and their electronic, magnetic and structural properties. Chapter 4 focuses on water splitting on manganites and electron and hole transfer through the chemical bonding. Chapter 5 discusses about hydrogen related defects in rutile titanium dioxide $\left(\mathrm{TiO}_{2}\right)$ and also metal/rutile interface. In chapter 6 , we make comprehensive discussion about the transition metal oxides, water splitting and particular challenges. Summary is the chapter 7 of the present thesis. For interested readers some computational details and theoretical derivations are provided in the appendices. 


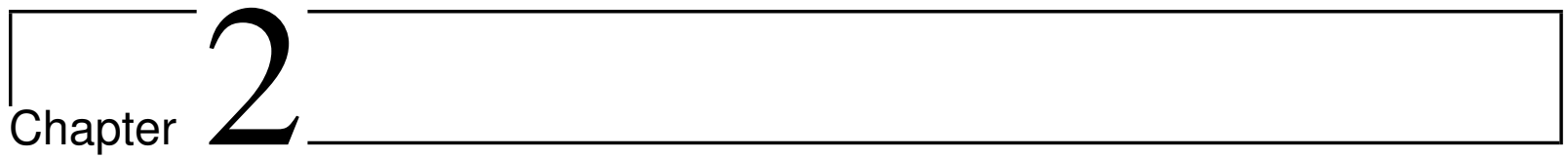

\section{Computational methods}

A significant part of condensed matter physics and chemistry would be calculating the electronic structure of atoms, molecules and solids. This however is a tough task for two main reasons. Firstly, electrons in matter must be treated using the laws of quantum mechanics rather than classical physics. The second problematic issue concerns the number of electrons that are involved. The complexity grows dramatically with increasing electron number. It is for these reasons that the electronic structure of matter is known as the quantum many-body problem. In this chapter, some relevant theories for solving manybody electronic structures and the other practicalities involved in performing total energy calculations are described. These include density functional theory, projector augmented wave method, basis sets, and molecular dynamics. These techniques and their combination are now very popular for the treatment of electronic structures of condensed phases.

\subsection{Born-Oppenheimer approximation}

The idea of Born-Oppenheimer approximation [47, 48] is to use the assumption that separates the electronic and nuclear coordinates. In this approximation, one first determines the electronic eigenstates for a set of nuclei with fixed atomic positions. Then, this solution is an starting point for the description of the nuclei dynamic. This description makes it possible and less complicated to compute the quantum mechanical treatment and then the energy of an entire system. From that energy the properties of the system are derived or computed. For many-body systems, such as a molecule or solid, containing a system of $N$ nuclei described by coordinates, $\mathbf{R}_{1}, \ldots, \mathbf{R}_{N} \equiv \mathbf{R}$, and masses $M_{1}, \ldots, M_{N}$, and $N_{e}$ electrons described by coordinates, $\mathbf{r}_{1}, \ldots, \mathbf{r}_{N_{e}} \equiv \mathbf{r}$, and spin variables, $s_{1}, \ldots, s_{N_{e}} \equiv s$, the Hamiltonian is given by 


$$
\begin{aligned}
\hat{H}= & \sum_{I=1}^{N} \frac{-\hbar^{2}}{2 M_{I}} \nabla_{\mathbf{R}_{I}}^{2}+\sum_{i=1}^{N_{e}} \frac{-\hbar^{2}}{2 m_{e}} \nabla_{\mathbf{r}_{i}}^{2}+\sum_{i>j} \frac{e^{2}}{\left|\mathbf{r}_{i}-\mathbf{r}_{j}\right|}+\sum_{I>J} \frac{Z_{I} Z_{J} e^{2}}{\left|\mathbf{R}_{I}-\mathbf{R}_{J}\right|}-\sum_{i, I} \frac{Z_{I} e^{2}}{\left|\mathbf{R}_{I}-\mathbf{r}_{i}\right|}(2.1) \\
& \equiv \hat{T}_{\mathrm{N}}(\mathbf{R})+\hat{T}_{\mathrm{e}}(\mathbf{r})+\hat{V}_{\mathrm{ee}}(\mathbf{r})+\hat{V}_{\mathrm{NN}}(\mathbf{R})+\hat{V}_{\mathrm{eN}}(\mathbf{r}, \mathbf{R})
\end{aligned}
$$

where $m_{e}$ and $Z_{I} e$ are the mass of the electron, and the charge on the $I$ th nucleus respectively. In the second line, the first term, $\hat{T}_{\mathrm{N}}$, describes the nuclear kinetic energy operator. The second term, $\hat{T}_{\mathrm{e}}$, represents the electron kinetic energy operator. The third term denoted by $\hat{V}_{\text {ee }}$, describes electron-electron interaction potential operator. The forth term, denoted by $\hat{V}_{\mathrm{NN}}$, represents nuclear-nuclear interaction potential operator. The last one, denoted by $\hat{V}_{\mathrm{eN}}$ describes electron-nuclear interaction potential operator. It should be noted that the interaction potential operators are the electrostatic repulsion either between the electrons or nuclei, and electrostatic attraction between electrons and nuclei. This Hamiltonian is the standard model to describe many-body systems, in particular atoms, molecules, and the more complex materials from proteins, enzymes, and nucleic acids, to metals and semiconductors. Thus, by solving and having the eigenvalues and eigenfunctions of this Hamiltonian, one could, predict the total energy of a given system. Of course, the problem cannot actually be solved exactly, and in order to make progress we need to simplify the problem. Thus, using approximation methods are needed and it is in the development of approximation methods and an analysis of their accuracy that solid state physics is focused.

To solve the complete quantum mechanical problem, the wave function of this Hamiltonian, Eq. 2.1, must be determined. The wave function will be given on the basis of the time-dependent Schrödinger equation

$$
i \hbar \frac{\partial}{\partial t}|\Psi(\mathbf{x}, \mathbf{R}, t)\rangle=\hat{H}|\Psi(\mathbf{x}, \mathbf{R}, t)\rangle
$$

where $\mathbf{x} \equiv(\mathbf{r}, s)$ combines the electron position and the spin variables into the full collection, and $|\Psi(\mathbf{x}, \mathbf{R}, t)\rangle$ which is an eigenfunction of Hamiltonian $\hat{H}$ with $E$ as an eigenvalue. In order to solve Eq. 2.2 for any systems, the Exponential Wall problem has to be resolved. This means, by increasing electron number $N_{e}$, number of configurations increase exponentially. Therefore, to simplify the problem, approximations must be applied. In a dynamical sense, by keeping the quantum nature of the electrons, treating the nuclei as classical particles will be a reasonable approximation. This can be used for the Eq. 2.2. According to the approach of Born and Huang [48], electronic Hamiltonian operator, $\hat{H}_{e}$ for fixed position of the nuclei are defined as

$$
\hat{H}_{e}(\mathbf{R})=\hat{T}_{\mathrm{e}}(\mathbf{r})+\hat{V}_{\mathrm{ee}}(\mathbf{r})+\hat{V}_{\mathrm{NN}}(\mathbf{R})+\hat{V}_{\mathrm{eN}}(\mathbf{r}, \mathbf{R})
$$


and it's eigenstate, $\phi_{k}(\mathbf{x}, \mathbf{R})$, which is also known as a Born-Oppenheimer wave function, can be given by

$$
\hat{H}_{e}(\mathbf{R}) \phi_{k}(\mathbf{x}, \mathbf{R})=E_{e}^{k} \phi_{k}(\mathbf{x}, \mathbf{R})
$$

where $E_{e}^{k}$ is the position-dependent energy eigenvalues of the electronic Hamiltonian, $H_{e}$. The ground state and many excited states are labeled by the quantum number $k$. Furthermore, each position-dependent electronic eigenvalue, $E_{e}^{k}(\mathbf{R})$, will give rise to an electronic surface which are known as Born-Oppenheimer surfaces. By solving the nuclear eigenvalue problem $\left(\hat{T}_{\mathrm{N}}+E_{e}^{k}\right)$ on each Born-Oppenheimer surface, a set of levels (rotational and vibrational) will be yielded. Since the electronic wave function, $\phi_{k}(\mathbf{x}, \mathbf{R})$, can generally be chosen to form a complete orthonormal basis set, the many particle wave function can assume as a quasi-separable ansatz of the form

$$
\Psi(\mathbf{x}, \mathbf{R})=\sum_{k} \phi_{k}(\mathbf{x}, \mathbf{R}) \chi_{k}(\mathbf{R}, t)
$$

where $\chi_{k}(\mathbf{R}, t)$ is a nuclear wave function and $\phi_{k}(\mathbf{x}, \mathbf{R})$ is an electronic wave function which depends parametrically on the nuclear positions. Eq. 2.5 for the wave function is not an approximation. Every wave function can be represented in this form. Substituting this expansion into the many-particle wave function, Eq. 2.2, and multiply the left side by the complex conjugate electronic wave function, $\phi_{k}^{*}(\mathbf{x}, \mathbf{R})$, and integrate over all electron coordinates, finally gets

$$
i \hbar \frac{\partial}{\partial t} \chi_{k}(\mathbf{R}, t)=\left[\hat{T}_{\mathrm{N}}+E_{e}^{k}(\mathbf{R})\right] \chi_{k}(\mathbf{R}, t)+\underbrace{\sum_{k^{\prime}}\left(c_{k, k^{\prime}}+\hat{A}_{k, k^{\prime}}\right) \chi_{k^{\prime}}(\mathbf{R}, t)}_{\text {non-adiabatic }}
$$

where the coefficients $c_{k, k^{\prime}}$ and the operators $\hat{A}_{k, k^{\prime}}$ are defined as

$$
\begin{aligned}
& c_{k, k^{\prime}}=\int d^{4} \mathbf{x}_{1} \cdots \int d^{4} \mathbf{x}_{N} \phi_{k}^{\star}(\mathbf{x}, \mathbf{R}) \hat{T}_{N} \phi_{k^{\prime}}(\mathbf{x}, \mathbf{R}) \\
& \hat{A}_{k, k^{\prime}}=\sum_{I=1}^{N} \frac{-\hbar^{2}}{M_{I}}\left(\int d^{4} \mathbf{x}_{1} \cdots \int d^{4} \mathbf{x}_{N} \phi_{k}^{*}(\mathbf{x}, \mathbf{R}) \nabla_{\mathbf{R}_{I}} \phi_{k^{\prime}}(\mathbf{x}, \mathbf{R})\right) \nabla_{\mathbf{R}_{I}} .
\end{aligned}
$$

It is clear that nuclei are heavier than electrons. For example, the proton is approximately 2000 times heavier an electron. This means that the electrons are in an eigenstate 
of $\hat{H}_{e}$ at all times that the movements of the atomic nuclei do not cause transitions between two electronic states $\phi_{k}(\mathbf{x}, \mathbf{R})$ and $\phi_{k^{\prime}}(\mathbf{x}, \mathbf{R})$. In the case that the different surfaces do not influence each other, the contribution of the non-adiabatic terms in Eq. 2.6 to the total energy is small and can be neglected. However, two Born-Oppenheimer sheets may be come so close that the non-adiabatic effects can no more be ignored. The neglect of non-adiabatic terms is the Born-Oppenheimer approximation. Thus, the dynamics of nuclei that move on the given total energy surface can be described by a time-dependent Schrödinger equation which has the time-dependent nuclear wave function. This wave function is denoted by $\chi_{k}(\mathbf{R}, t)$ which applies to the equation below:

$$
i \hbar \frac{\partial}{\partial t} \chi_{k}(\mathbf{R}, t)=\left[\hat{T}_{\mathrm{N}}+E_{e}^{k}(\mathbf{R})\right] \chi_{k}(\mathbf{R}, t)
$$

In other words, a Schrödinger equation for the motion of the nuclei are independent of the electronic excitation and for the system in the electronic ground state, it will remain in the same electronic ground state. Since Eq. 2.4 depends parametrically on the nuclear coordinates and thus represents a time-independent Schrödinger equation for the electrons alone, electron and nuclear motion are decoupled from each other in the Born-Oppenheimer approximation. So, the problem of solving $\left(3 N+3 N_{e}+1\right)$-dimensional partial differential equation has been reduced to the solution of lower dimensionality, a $3 N_{e}$ dimensional for the electrons and a $3 N+1$ dimensional for the atomic nuclei. However, even these equations must be further simplified in order to be able to actually solve them.

Ignoring the gradient terms of the electronic wave function $-i \hbar\left\langle\phi_{k^{\prime}}(\mathbf{x}, \mathbf{R})\left|\nabla_{\mathbf{R}_{I}}\right| \phi_{k^{\prime}}(\mathbf{x}, \mathbf{R})\right\rangle$ which is so-called derivative coupling, is an approximation. In other word, the neglect of the non-adiabatic effects is the essence of the Born-Oppenheimer approximation. Imagine a system with the electrons in the ground state energy $E_{e}^{0}(\mathbf{R})$. At temperature $T$, the first excited state is denoted by $E_{e}^{1}(\mathbf{R})$. There is no excitation to this state if $\left|E_{e}^{1}(\mathbf{R})-E_{e}^{0}(\mathbf{R})\right| \gg k T$. But, sometimes there could be regions where the surfaces are close to each other with an energy spacing close to $k T$. If the system visits such nuclear configurations, then the Born-Oppenheimer approximation is not more valid and it will break down. However, in the case that Born-Oppenheimer surfaces never come close neither even cross, non-adiabatic effects can be neglected, and motion is considered only on the ground electronic surface which will be described by these two equations:

$$
\begin{aligned}
& {\left[T_{\mathrm{e}}+V_{\mathrm{ee}}(\mathbf{r})+V_{\mathrm{eN}}(\mathbf{r}, \mathbf{R})+V_{\mathrm{NN}}(\mathbf{R})\right] \phi_{0}(\mathbf{x}, \mathbf{R})=E_{e}^{0}(\mathbf{R}) \phi_{0}(\mathbf{x}, \mathbf{R})} \\
& {\left[T_{\mathrm{N}}+E_{e}^{0}(\mathbf{R})\right] \chi_{0}(\mathbf{R}, t)=i \hbar \frac{\partial}{\partial t} \chi_{0}(\mathbf{R}, t)}
\end{aligned}
$$

\subsubsection{Classical approximation}

The neglect of the nuclear quantum effects, can lead to the classical motion on the groundstate surface which is equivalent to the Hamilton-Jacobi equation. This motion is govern 
by:

$$
\begin{aligned}
\dot{\mathbf{R}}_{I} & =\frac{\mathbf{P}_{I}}{M_{I}} \\
\dot{\mathbf{P}}_{I} & =-\nabla_{I} E_{0}(\mathbf{R})
\end{aligned}
$$

where $\mathbf{P}_{I}$ is the momenta of nuclei and $E_{0}(\mathbf{R})$, is the ground-state surface energy. The latter equation can be rewritten in the different form because of the Hellman-Feynman theorem as following:

$$
\nabla_{\mathbf{R}_{I}} E_{0}(\mathbf{R})=\left\langle\phi_{0}(\mathbf{R})\left|\nabla_{\mathbf{R}_{I}} \hat{H}_{\mathrm{e}}(\mathbf{R})\right| \phi_{0}(\mathbf{R})\right\rangle
$$

Eq. 2.10 and 2.12 form the theoretical basis of the most molecular dynamic approaches. However, the dynamic of the nuclei requires the knowledge of the electronic problem. The difficulty is to obtain Born-Oppenheimer wave functions particularly the ground state electronic wave function, $\left|\phi_{0}(\mathbf{R})\right\rangle$ and eigenvalue, $E_{e}^{0}(\mathbf{R})$. In general, It is impossible to find an exact solution for the electronic wave function and eigenvalue and it is necessary to apply an approximation scheme for obtaining these quantities. This is the subject of the next section.

\section{$2.2 \quad$ Variational method}

Since a direct solution for Eq. 2.4 is impossible, an approximation method for solving the eigenvalue problem can be found. The basis of this method is variational principle. It can be shown that Eq. 2.4 is equivalent to the problem of the extremum of the energy functional

$$
E[\phi]=\frac{\left\langle\phi\left|\hat{H}_{e}\right| \phi\right\rangle}{\langle\phi \mid \phi\rangle}
$$

with respect to any allowable test to find wave function $\phi$, which must be antisymmetric with respect to the interchange of two electrons and must satisfy the constraints imposed by system under consideration. So, for the variation of $E[\phi]$ must have

$$
\delta\left\{\frac{\left\langle\phi\left|\hat{H}_{e}\right| \phi\right\rangle}{\langle\phi \mid \phi\rangle}\right\}=0 .
$$

If $E_{e}^{0}$ is the lowest eigenvalue of $\hat{H}_{e}$, ground state energy, and $\phi_{0}$ is the associated eigenfunction (ground state wave function), then 


$$
E[\phi] \geq E_{e}^{0}=\frac{\left\langle\phi_{0}\left|\hat{H}_{e}\right| \phi_{0}\right\rangle}{\left\langle\phi_{0} \mid \phi_{0}\right\rangle}
$$

where $E=E_{e}^{0}$ if and only if $\phi=\phi_{0}$. By minimizing $E[\phi]$, it is thus possible to obtain exact ground state energy and wave function of an electron system. This ansatz of the Schrödinger equation still has no simplification of the problem, but it lays the foundation for many approximation methods for the determination of ground state properties.

A class of approximation methods does not look for the ground state function in the Hilbert space of all possible wave functions, but limited to a particular subspace. For example the Hartree-Fock method considers only those many-body wave functions that can be represented by a single Slater determinant [49]. The ground state wave function of the Configuration-Interaction-Method (CI) [49] is an energy minimizing linear combination of Slater-determinant.

This is based on the wave-function-based electron-structure methods and it allows an increasing in their accuracy by expanding the space of the test wave function. However, this accuracy is paid for by a very high computational efforts. So, by using current computer systems, only small amount of systems can be calculated in the reasonable time. To describe even more complex system, Density Functional Theory (DFT) offers the possibly of mapping the fully interacting many-body problem onto non-interacting many particle problem, thereby greatly reducing the computational burden.

\subsection{Density-Functional Theory}

Density-functional theory $[50,51]$ is presently a computational quantum mechanical approach to compute the electronic structure of many-body system in particular atoms, molecules, and the condensed phases. The density-functional theory is based on an exact theorem. The ground-state properties of a system is investigated by the spatially dependent electron density. DFT predicts a great variety of molecular properties: molecular structures, vibrational frequencies, atomization energies, ionization energies, electric and magnetic properties, reaction paths, etc. It should be noted that the original theorems held only for non-degenerate ground states in the absence of a magnetic field.

The starting point of the theory is the fact that electron density contains in principle all the information contained in the many-body ground state wave function. The electronic density of many-electron system is defined to be

$$
n(\mathbf{r})=\sum_{i=1}^{N_{e}} \int d^{4} \mathbf{x}_{1} \ldots \int d^{4} \mathbf{x}_{N_{e}}\left\langle\phi \mid \mathbf{x}_{1} \ldots \mathbf{x}_{N_{e}}\right\rangle \delta\left(\mathbf{x}-\mathbf{x}_{i}\right)\left\langle\mathbf{x}_{1} \ldots \mathbf{x}_{N_{e}} \mid \phi\right\rangle
$$


All $N_{e}$-electron wave functions $\phi\left(\mathbf{x}_{1}, \ldots, \mathbf{x}_{N_{e}}\right)$ obey the Pauli principle, that means when two particles are exchanged, the sign of the wave function will change. In this notation, $\mathbf{x} \equiv(\mathbf{r}, s)$ which is combination of the electron position and the spin index. Similarly, the notation of a four-dimensional integral $\int d^{4} \mathbf{x}:=\sum_{s} \int d^{3} \mathbf{r}$ is used for the sum over spin indices and the integral over the position as well as, $\delta\left(\mathbf{x}-\mathbf{x}^{\prime}\right):=\delta_{s, s^{\prime}} \delta\left(\mathbf{r}-\mathbf{r}^{\prime}\right)$, which denotes the product of Kronecker delta of the spin coordinates and Dirac's delta function for the positions.

In 1964, Hohenberg and Kohn [50] pointed out that all properties of a system defined by an external potential $V_{\mathrm{eN}}$ which can be clearly determined from the density of the ground state $n(\mathbf{r})$. The ground state energy is thus a functional of the density $n(\mathbf{r})$.

$$
E[n(\mathbf{r})]=T_{\mathrm{e}}+V_{\mathrm{ee}}+\int d^{3} \mathbf{r} n(\mathbf{r}) V_{\mathrm{eN}}+V_{\mathrm{NN}},
$$

where $T_{\mathrm{e}}, V_{\mathrm{ee}}, V_{\mathrm{eN}}$, and $V_{\mathrm{NN}}$ have analogous meaning to the operators defined in Eq. 2.1. Regarding to the variational principle formulated for wave functions, Eq. 2.14 and 2.15, this functional reaches its minimum with respect to all allowed test densities if the test density is identical to the ground state density $n(\mathbf{r})=n_{0}(\mathbf{r})$. A closer look at Eq. 2.17 shows that only $V_{\mathrm{eN}}$ and $V_{\mathrm{NN}}$ depend on the system, while $T_{\mathrm{e}}+V_{\mathrm{ee}}$ is system-independent and thus universally valid. If one knows the explicit form of the universal functional $T_{\mathrm{e}}+V_{\mathrm{ee}}$, then exact solution of Schrödinger equation is available for the ground state of any system.

The first theorem demonstrates that all ground-state properties are unique functionals of the electron density which depends on only 3 spatial coordinates. By using electron density, the many-body problem of $\mathrm{N}$ electrons with $3 \mathrm{~N}$ spatial coordinates reduces to 3 spatial coordinates. This theorem has been extended to the time-dependent domain to develop time-dependent density functional theory (TDDFT), which can be used to describe excited states.

To prove the claim, suppose it is false and there exist two external potentials $V_{\mathrm{eN}}^{1}$ and $V_{\mathrm{eN}}^{2}$ that results in the same charge density. The Hamiltonian that result from them are $H_{e}^{1}$ and $H_{e}^{2}$, and the corresponding wave functions are $\phi^{1}$ and $\phi^{2}$, respectively. Thus, the ground state energy of $H_{e}^{1}$ is realized only by $\phi^{1}$. Assume that the ground state the two Hamiltonian are non-degenerate. So,

$$
E_{e}^{1}=\left\langle\phi^{1}\left|H_{e}^{1}\right| \phi^{1}\right\rangle<\left\langle\phi^{2}\left|H_{e}^{1}\right| \phi^{2}\right\rangle
$$

Because $\phi^{2}$ is not the ground state of $H_{e}^{1}$. So, by knowing that two Hamiltonian with the same number of electrons can differ only in the potential, one can rewrite,

$$
\begin{aligned}
& E_{e}^{1}<\left\langle\phi^{2}\left|H_{e}^{2}\right| \phi^{2}\right\rangle+\left\langle\phi^{2}\left|\left(H_{e}^{1}-H_{e}^{2}\right)\right| \phi^{2}\right\rangle \\
& \Rightarrow E_{e}^{1}<E_{e}^{2}+\int d \mathbf{r} n(\mathbf{r})\left[V_{\mathrm{eN}}^{1}-V_{\mathrm{eN}}^{2}\right] .
\end{aligned}
$$


However, one can switch indices 1 and 2 to obtain,

$$
E_{e}^{2}<E_{e}^{1}+\int d \mathbf{r} n(\mathbf{r})\left[V_{\mathrm{eN}}^{2}-V_{\mathrm{eN}}^{1}\right]
$$

Adding Eq. 2.19 and 2.22 gives

$$
E_{e}^{2}+E_{e}^{1}<E_{e}^{2}+E_{e}^{1}
$$

which is a contradiction. Therefore, $V_{\mathrm{eN}}^{1}$ and $V_{\mathrm{eN}}^{2}$ must be the same. As a result, by having the charge density, one can therefore deduce $V_{\mathrm{eN}}$ and solve for all properties of the system.

The second theorem defines that the electron density can be obtained from a Schrödinger equation in an effective potential. In other word, the correct ground-state electron density, minimizes the energy functional.

This assertion is proved by noting that if one starts with the wrong density, $n^{2}(\mathbf{r})$, for $H_{e}^{1}$, then $n^{2}(\mathbf{r})$ should really be associated with a different Hamiltonian $H_{e}^{2}$, which has ground state wave function $\phi_{2}$ and which does not minimize

$$
\left\langle\phi_{2}\left|H_{e}^{1}\right| \phi_{2}\right\rangle=E_{e}^{1}\left[n^{2}(\mathbf{r})\right]
$$

Only $n^{1}(\mathbf{r})$ minimizes $E_{e}^{1}\left[n^{1}(\mathbf{r})\right]$. The most important feature of this view of the manybody problem is that one can write the energy functional like Eq. 2.17 where $T_{\mathrm{e}}$ and $V_{\mathrm{ee}}$ do not depend upon the potential $V_{\mathrm{eN}}$. If one could find this functional, it would solve all many-body problems for all external potential $V_{\mathrm{eN}}$.

\subsubsection{Kohn-Sham equations}

Unfortunately, the exact functional dependence of these functionalities on the density is not known. To overcome this problem, and in particular to facilitate the description of kinetic energy, these theorems were further developed by Walter Kohn and Lu Jeu Sham [51] to produce Kohn-Sham DFT (KS DFT). Within this framework, interacting electron system maps onto a system of non-interacting electrons in an effective potential. The effective potential depends on the electron distribution and describes external potential and the effects of the Coulomb interactions between the electrons (the exchange and correlation interactions). Since finding of the latter two interaction is difficult, approximations permit to calculate certain physical quantities. DFT provides a ground-state total energy which has the form 


$$
\begin{aligned}
E[n(\mathbf{r})]=\sum_{n} f_{n}\left\langle\phi_{n}\left|\frac{\mathbf{p}^{2}}{2 m}\right| \phi_{n}\right\rangle+ & \int d^{3} \mathbf{r} v_{e x t}(\mathbf{r}) n(\mathbf{r}) \\
& +\frac{1}{2} \int d^{3} \mathbf{r} \int d^{3} \mathbf{r}^{\prime} \frac{e^{2} n(\mathbf{r}) n\left(\mathbf{r}^{\prime}\right)}{4 \pi \epsilon_{0}\left|\mathbf{r}-\mathbf{r}^{\prime}\right|}+E_{x c}[n(\mathbf{r})] .
\end{aligned}
$$

The first term is the kinetic energy of the non-interacting many electron system with density $n(\mathbf{r})$. The second one denotes the external potential. The third one expresses the Hartree energy. The last one is the exchange and correlation functional, $E_{x c}[n(\mathbf{r})]$, contains Coulomb interactions, which is not known in practice. Therefore, exchange-correlation term needs to be expressed by approximate functionals. Here the electron density constructed as:

$$
n(\mathbf{r})=\sum_{s} \sum_{n} f_{n}\left|\phi_{n}(\mathbf{x})\right|^{2}
$$

where $n$ refers to single-particle states and the sum is over all the occupied states. It should be noted that the non-interacting system can be defined by single-particle equations and the ground-state solution is the corresponding Slater determinant. As a result of the minimum principle, the following Kohn-Sham equation for the one particle orbital has the form

$$
\left[\frac{\mathbf{p}^{2}}{2 m}+v_{e f f}(\mathbf{r})-\varepsilon_{n}\right]\left|\phi_{n}\right\rangle=0
$$

where the effective potential, $v_{\text {eff }}(\mathbf{r})$, is defined as

$$
v_{e f f}(\mathbf{r})=v_{e x t}(\mathbf{r})+\frac{e^{2}}{4 \pi \epsilon_{0}} \int d^{3} \mathbf{r}^{\prime} \frac{n\left(\mathbf{r}^{\prime}\right)}{\left|\mathbf{r}-\mathbf{r}^{\prime}\right|}+\frac{\delta E_{x c}}{\delta n(\mathbf{r})}
$$

where the first term is the local ionic potential or external potential. The second one is the Hartree potential. The last term is the exchange-correlation potential. Once Eq. 2.26 are solved self-consistently (the solution are denoted by $\tilde{\phi}_{n}$ ), we obtain the ground state density:

$$
n_{0}(\mathbf{r})=\sum_{s} \sum_{n} f_{n}\left|\tilde{\phi}_{n}(\mathbf{x})\right|^{2}
$$


and the total ground state energy is given by:

$$
\begin{aligned}
E_{0}\left[n_{0}(\mathbf{r})\right]=\sum_{n} f_{n}\left\langle\tilde{\phi}_{n}\left|\frac{\mathbf{p}^{2}}{2 m}\right| \tilde{\phi}_{n}\right\rangle+ & \int d^{3} \mathbf{r} v_{e x t}(\mathbf{r}) n_{0}(\mathbf{r}) \\
& +\frac{1}{2} \int d^{3} \mathbf{r} \int d^{3} \mathbf{r}^{\prime} \frac{e^{2} n_{0}(\mathbf{r}) n_{0}\left(\mathbf{r}^{\prime}\right)}{4 \pi \epsilon_{0}\left|\mathbf{r}-\mathbf{r}^{\prime}\right|}+E_{x c}\left[n_{0}(\mathbf{r})\right]
\end{aligned}
$$

Note that the preceding result for total energy does not include the Nuclei interaction, $V_{\mathrm{NN}}$. For a given ion configuration, this term is a constant. However, when we move the ions and calculate the forces on them, we need to take this term into account. Several approaches are developed to solve this type of equations that, in the following we try to introduce the basic parts of DFT and provide some guidance into approximations.

\subsubsection{Exchange-correlation functional}

In a classical description, which has uncorrelated electrons, the probability of finding the pair of electrons at the positions $\mathbf{r}$ and $\mathbf{r}^{\prime}$ is simply given by $n^{(2)}\left(\mathbf{r}, \mathbf{r}^{\prime}\right)=n^{(1)}(\mathbf{r}) n^{(1)}\left(\mathbf{r}^{\prime}\right)$ where $n^{(2)}\left(\mathbf{r}, \mathbf{r}^{\prime}\right)$ is the two particle density and $n^{(1)}(\mathbf{r})$ is the total electron density. It shows the classical Coulomb repulsion, or Hartree energy. But in reality, electrons are correlated and they obey Fermi statistics. Moreover, electrons kept apart by the Pauli-exclusion principle. Therefore, these exchange and correlation interactions affects the electron density. The electron density at position $\mathbf{r}$ reduces compare to the classical value, because of the instantaneous position of the second electron located at $\mathbf{r}^{\prime}$. Hence, each electron creates a depletion, or let say hole, of electron density around itself. This is a direct consequence of exchange-correlation effects. The two-particle density can now be written by the density and the exchange-correlation hole function as,

$$
n^{(2)}\left(\mathbf{r}, \mathbf{r}^{\prime}\right)=n^{(1)}(\mathbf{r}) n^{(1)}\left(\mathbf{r}^{\prime}\right)+n^{(1)}(\mathbf{r}) h\left(\mathbf{r}, \mathbf{r}^{\prime}\right)
$$

where the quantum effects are in the exchange-correlation hole density, $h\left(\mathbf{r}, \mathbf{r}^{\prime}\right)$. It also affects the Coulomb repulsion between the electrons, by the attraction of the electron to its exchange-correlation hole. Coulomb repulsion is reduced relative to the Hartree energy by this effect. The exchange-correlation hole satisfies an important normalization condition known as a sum rule,

$$
\int d \mathbf{r}^{\prime} h\left(\mathbf{r}, \mathbf{r}^{\prime}\right)=-1
$$

This implies that an electron in an $N$-electron system, always sees $N-1$ other electrons. In other word, the exchange-correlation hole has a deficit of exactly one electron. 
Now, the interaction energy can be described by the two-particle density as follows,

$$
V_{\mathrm{ee}}=\frac{1}{2} \int d^{3} \mathbf{r} \int d^{3} \mathbf{r}^{\prime} \frac{e^{2} n^{(2)}\left(\mathbf{r}, \mathbf{r}^{\prime}\right)}{4 \pi \epsilon_{0}\left|\mathbf{r}-\mathbf{r}^{\prime}\right|}
$$

where, $n^{(2)}\left(\mathbf{r}, \mathbf{r}^{\prime}\right)$ is the two-particle reduced density matrix (see Appendix. A). It can be calculated as

$$
n^{(2)}\left(\mathbf{r}, \mathbf{r}^{\prime}\right):=N(N-1) \sum_{s, s^{\prime}} \int d^{4} \mathbf{x}_{3} \cdots \int d^{4} \mathbf{x}_{N}\left|\phi\left(\mathbf{x}, \mathbf{x}^{\prime}, \mathbf{x}_{3}, \ldots, \mathbf{x}_{N}\right)\right|^{2}
$$

According to Eq. 2.30, the Coulomb interaction between electrons can be written by two terms. The first part is the so-called Hartree energy

$$
E_{H}=\frac{1}{2} \int d^{3} \mathbf{r} \int d^{3} \mathbf{r}^{\prime} \frac{e^{2} n^{(1)}(\mathbf{r}) n^{(1)}\left(\mathbf{r}^{\prime}\right)}{4 \pi \epsilon_{0}\left|\mathbf{r}-\mathbf{r}^{\prime}\right|}
$$

and the second part is the potential energy of exchange and correlation

$$
U_{x c}=\int d^{3} \mathbf{r} n^{(1)}(\mathbf{r}) \frac{1}{2} \int d^{3} \mathbf{r}^{\prime} \frac{e^{2} h\left(\mathbf{r}, \mathbf{r}^{\prime}\right)}{4 \pi \epsilon_{0}\left|\mathbf{r}-\mathbf{r}^{\prime}\right|}
$$

Note that $U_{x c}$ is not the exchange-correlation energy $\left(E_{x c}\right)$. The difference between these two quantities is a kinetic energy correction. In order to facilitate the calculation of the kinetic energy, the interacting electron system maps onto a non-interacting electrons with the same density as a true system $\left(T_{s}\left(n^{(1)}\right)\right)$. The difference relative to the real system is added to the potential energy of exchange and correlation. Thus, the exchange-correlation energy can be expressed as

$$
E_{x c}=U_{x c}+\sum_{n} \bar{f}_{n} \int d^{4} \mathbf{x} \varphi_{n}^{*}(\mathbf{x}) \frac{-\hbar^{2}}{2 m} \nabla^{2} \varphi_{n}(\mathbf{x})-T_{s}\left(n^{(1)}\right)
$$

In fact, this is shown that the exchange-correlation functional can be written as a functional of the electron density which is available to the seminal paper by Levy [52]. Note that the $\varphi_{n}(\mathbf{x})$ and the $\bar{f}_{n}$ are natural orbitals and occupation of the interacting electron gas, respectively. They are completely different from the Kohn-Sham orbitals $\phi_{n}(\mathbf{x})$ and occupations $f_{n}$. 


\section{Exact definition of exchange and correlation}

So far one can estimate the exchange-correlation energy, however, in DFT an exact definition for this functional, can be derived using a method known as adiabatic connection $[53,54,55]$. The basic concept is that while the density is fixed, the non-interacting system can be connected to the interacting system. This connection is carried out by a coupling-constant $\lambda$. When $\lambda=0$, the non-interacting system arises, and $\lambda=1$ represents the fully interacting system. The exchange-correlation hole, $h\left(\mathbf{r}, \mathbf{r}^{\prime}\right)$, can be written as an average over a coupling-constant dependent hole

$$
\bar{h}_{x c}\left(\mathbf{r}, \mathbf{r}^{\prime}\right)=\int_{0}^{1} h^{\lambda}\left(\mathbf{r}, \mathbf{r}^{\prime}\right) d \lambda
$$

A useful quantity to define from this is the exchange-correlation energy per electron, $\varepsilon_{x c}[n(\mathbf{r})]$, which itself is a functional of the density. It is also known as energy density,

$$
\varepsilon_{x c}[n(\mathbf{r})]=\frac{1}{2} \int \frac{e^{2} \bar{h}_{x c}\left(\mathbf{r}, \mathbf{r}^{\prime}\right)}{4 \pi \epsilon_{0}\left|\mathbf{r}-\mathbf{r}^{\prime}\right|} d^{3} \mathbf{r}^{\prime}
$$

If this parameter, $h\left(\mathbf{r}, \mathbf{r}^{\prime}\right)$, exists in an exactly form, simply the electron many-particle problem would be solved. The adiabatic connection method also provides another important result. The difference between the interacting and non-interacting kinetic energy, $T_{c}[n(\mathbf{r})]=T[n(\mathbf{r})]-T_{s}[n(\mathbf{r})]$, is included within the definition $\bar{h}_{x c}\left(\mathbf{r}, \mathbf{r}^{\prime}\right)$.

\section{Self-interaction effects}

The self-interaction can be defined for a system containing exactly one electron. In this case the Hartree term (Eq. 2.34) is not zero for a one electron system. Imagine one of the spin densities is zero, let say $n_{\beta}(\mathbf{r})=0$, then,

$$
\int n_{\alpha}(\mathbf{r}) d \mathbf{r}=\int n(\mathbf{r}) d \mathbf{r}=1
$$

Since, there is no probability of finding another electron in the system, the exchangecorrelation hole and the density must add to zero at all points in space. So, there are no electron-electron interaction, which leads to the result,

$$
E_{x c}\left[n_{\alpha}(\mathbf{r}), 0\right]=-E_{H}[n(\mathbf{r})], \quad E_{C}\left[n_{\alpha}(\mathbf{r}), 0\right]=0
$$


where $E_{C}$ is a correlation energy. Thus, the self-energy of the electron is canceled by exchange. In another word, the exchange-correlation energy term directly cancels the Hartree energy of the system. Nearly all of the conventional functionals in DFT contains a self-interaction error $E^{S I E}$ in the computed total energies,

$$
E^{S I E}=E_{H}[n(\mathbf{r})]+E_{x c}\left[n_{\alpha}(\mathbf{r}), 0\right]
$$

This equation can be used as a measure of the degree of self-interaction exhibited by a given exchange-correlation functionals.

\subsubsection{Local density approximation (LDA)}

The first approximation used in practice is the local-density approximation (LDA), which is based upon exact exchange energy for a homogeneous electron gas with the same density. This is the most important functional which was proposed by Hohenberg and Kohn in their original DFT paper [50]. The LDA is only dependent on the local density, and the exchange-correlation energy can be written as,

$$
E_{x c}^{L D A}[n(\mathbf{r})]=\int n(\mathbf{r}) \varepsilon_{x c}^{h o m}[n(\mathbf{r})] d \mathbf{r},
$$

where $\varepsilon_{x c}^{\text {hom }}[n(\mathbf{r})]$ is the exchange-correlation energy per electron corresponding to an interacting homogeneous electron gas of density $n(\mathbf{r})$. A commonly used correlation formula has been parameterized by Perdew and Zunger [56] which uses accurate quantum Monte Carlo data of the homogeneous electron gas generated by Ceperley and Alder [57], to fix the coefficients in the interpolation formula. Despite its simplicity, the LDA works well for solid systems and has been used in solid state calculations for many years, however its success does not transfer to chemistry. The LDA has a notorious tendency to overbind, and is particularly severe for molecules.

\subsubsection{Generalized gradient approximation (GGA)}

Being concerned about the large density variations in the real materials opens up the first terms of a Taylor expansion in the density gradients which lead to the class of generalized gradient approximations (GGA) [58]. The GGA is currently the most popular exchangecorrelation functional in condensed matter physics. This functional modifies the LDA energy density by the enhancement factor, $F_{x c}[n(\mathbf{r}), \nabla n(\mathbf{r})]$. The GGA exchange-correlation can be written as,

$$
E_{x c}^{G G A}[n(\mathbf{r})]=\int n(\mathbf{r}) \varepsilon_{x c}^{h o m}[n(\mathbf{r})] F_{x c}[n(\mathbf{r}), \nabla n(\mathbf{r})] d \mathbf{r}
$$


Usually the GGA enhancement factor contains two terms. The first one is the Seitz radius $r_{s}$, and the second one is the dimensionless reduced density gradient $s(\mathbf{r})$, which has a form of,

$$
s(\mathbf{r})=\frac{|\nabla n(\mathbf{r})|}{2 k_{F}(\mathbf{r}) n(\mathbf{r})}
$$

where $k_{F}$ is the Fermi-wavevector,

$$
k_{F}(\mathbf{r})=\left[3 \pi^{2} n(\mathbf{r})\right]^{1 / 3}
$$

The GGA functional has a large variety of different forms, So, plotting $F_{x c}\left(r_{s}, s\right)$ against $s$ for various $r_{s}$ values is an effective way of examining and comparing different GGA functionals. GGA improved overbinding error for solids and molecules over the LDA functional. These GGA functionals also improved the bond energies and made DFT useful also for chemists. The most widely used GGA functional is the Perdew-Burke-Ernzerhof (PBE) functional [59]. It would be fair to say that the success of the GGA for molecular properties was a major factor in the part awarding of the Nobel Prize in chemistry to Kohn in 1998.

\subsubsection{Hybrid functionals}

An interesting class of functionals are hybrids [21, 60], which replace some of the conventional GGAs exchange by the exact (Hartree-Fock) exchange. The general form is

$$
E_{x c}^{h y b r i d}=\alpha\left(E_{x}^{H F}-E_{x}^{G G A}\right)+E_{x c}^{G G A},
$$

where $E_{x}^{H F}$ is the Hartree-Fock exchange expression. The coefficient, $\alpha$, is a so-called weight factor which determines the amount of exact-exchange mixing. This quantity can be fitted semi-empirically. This approach was put forward by Becke [21] who connected noninteracting system $(\lambda=0)$ to the fully-interacting system $(\lambda=1)$ through the adiabatic connection integral for the exact exchange-correlation energy. It could be approximated as:

$$
E_{x c}=\int_{0}^{1} U^{\lambda} d \lambda=\frac{1}{2} U^{0}+\frac{1}{2} U^{1}=\frac{1}{2} E_{x}^{H F}+\frac{1}{2} E_{x c, \lambda=1}^{D F T} .
$$

where $E_{x c, \lambda=1}^{D F T}$ is obtained from a density functional approximation such as the LDA or GGA. Since $\lambda=0$ corresponds only to the exchange, this can be defined by Hartree-Fock 
theory, while $\lambda=1$ represents the electron interactions, because of correlation, and so could be provided by a local-type density functional treatment. As a result, Becke suggested the so-called half-and-half functional. Depending on whether the $\lambda$-dependence is a straight line or whether it is convex, the weight factor may be equal or smaller than $\frac{1}{2}$. After that, Perdew [61] claimed that the optimum amount of exchange mixing factor should be reduced to $\frac{1}{4}$ on the basis of GGA.

Hybrids give significant improvement over GGAs for binding energies, band gaps and reaction energies, Thus, they are a very popular functional in quantum chemistry. The most widely used hybrid functional is the B3LYP functional proposed by Stephens et al. [62]. This functional is a generalisation of the B3P86 form devised by Becke [60].

\subsubsection{Local hybrid functional (PBE0r)}

In the local version of hybrid functional, PBE0r [63], a fraction of the exchange energy $E_{x}^{P B E}$ of the PBE [59] functional replaces by the explicit nonlocal Hartree-Fock exchange energy $E_{x}^{H F}$. PBE0r is derived from the PBE0 [64] functional. In the PBE0r, the KohnSham wave functions are mapped onto localized tight-binding orbitals and only the onsite exchange terms of the exchange correction are included. In this functional, the Fock term is limited to onsite terms in a carefully crafted local orbital basisset. This makes the computational cost of hybrid calculations comparable to that of regular GGA calculations.

$$
E^{P B E 0 r}=E^{P B E}+a_{x}\left(E_{x}^{H F, r}-E_{x}^{P B E, r}\right),
$$

where, $E_{x}^{H F, r}$ and $E_{x}^{P B E, r}$ are the Hartree-Fock exchange energy and the PBE exchange energy, respectively, with only consideration of the exchange for specified sets of local orbitals. The commonly used value a for the admixture in the PBE0 functional is $\mathrm{a}=0.25$ [61]. The non-local exchange term induces a stabilization of states with the same orbital character states that are occupied. Thus it correctly opens a band gap of transition metal oxides, that would vanish without this exchange correction. For this reason, the inclusion is vital for the present work. In contrast to other implementations of local hybrid functionals, $[65,66]$ we include the correction on all atoms and we take into account all tight-binding orbitals that nominally contribute the valence electrons and the core wave functions. Also the exchange interaction between core and valence electrons is included in the correction. We found that the commonly used correction factor of $a=0.25$ makes the physical realized configuration of f-electron atoms unstable. In the present work, we adjust these parameters such that the experimentally observed spectral features are well described.

The advantage of the local approach is that the mixing parameter can be chosen individually for each atom, reflecting an effective local dielectric constant. Furthermore, the Fock term on all atoms is taken into account for all tight-binding orbitals that contribute the valence electrons or the core wave functions. Also the exchange interaction between core and valence electrons is taken into account. The rationale for the inclusion of all orbitals 
is to avoid unphysical energy shifts between orbitals that are included in the correction relative to those that are not.

Like the augmentation, also the construction of local orbitals uses the framework of nodeless partial waves described elsewhere [67]. The local orbitals are constructed in the spirit of the tight-binding linear muffin-tin orbital (LMTO) method [68] by enforcing that only the nodeless scattering partial waves are admitted on the neighboring sites [67]. These orbitals are approximated by a one-center expansion and truncated beyond a radius of two times the covalent radius of that atom.

The orbital set includes states up to $5 s, 5 p, 5 d, 4 f$ for $\mathrm{Pr}$, up to $3 s, 3 p$ for Ca, up to $4 s, 3 p, 3 d$ for $\mathrm{Mn}$ and up to $2 s, 2 p$ for $\mathrm{O}$ atoms.

\subsubsection{Benchmarks}

It is very important to know the fact that practical DFT calculations are not exact solutions of the Schrödinger equation. So, there is an intrinsic uncertainty between the energy calculated with DFT and the true ground state energies. Furthermore, despite recent improvements, there are still difficulties in using DFT to properly describe interactions like van der Waals, excitations, and transition states. Moreover, standard DFT calculations have limited accuracy for band gaps, comparing to experimental data. The development of new DFT functionals designed to overcome this problem. The hybrid and local-hybrid functionals have been shown a good consistency with true band gaps which is used in a current research topic $[63,66]$.

\subsection{Projector augmented wave method}

The projector augmented wave method (PAW) was first proposed and implemented by Blöchl [69], as a generalization of ideas of both pseudopotential [70] and linear augmentedwave methods [71]. It is a technique for ab-initio electronic structure calculations, and allows DFT calculations to be performed with greater computational efficiency.

In the PAW method, the wavefunction $\psi_{n}$ can be derived from the smooth wavefunction $\tilde{\psi}_{n}$ by using a linear transformation operator $T$,

$$
\begin{aligned}
\left|\psi_{n}\right\rangle=T\left|\tilde{\psi}_{n}\right\rangle= & \left|\tilde{\psi}_{n}\right\rangle+\sum_{a} \sum_{i}\left(\left|\phi_{i}^{a}\right\rangle-\left|\tilde{\phi}_{i}^{a}\right\rangle\right)\left\langle\tilde{p}_{i}^{a} \mid \tilde{\psi}_{n}\right\rangle \\
& =\left|\tilde{\psi}_{n}\right\rangle+\sum_{a}\left(\sum_{i}\left|\phi_{i}^{a}\right\rangle\left\langle\tilde{p}_{i}^{a} \mid \tilde{\psi}_{n}\right\rangle-\sum_{i}\left|\tilde{\phi}_{i}^{a}\right\rangle\left\langle\tilde{p}_{i}^{a} \mid \tilde{\psi}_{n}\right\rangle\right)
\end{aligned}
$$

where $\left|\phi_{i}^{a}\right\rangle$ is a complete basis inside the augmentation sphere, and $\left|\tilde{\phi}_{i}^{a}\right\rangle$ is the smooth partial waves can be described by $\left|\phi_{i}^{a}\right\rangle=T\left|\tilde{\phi}_{i}^{a}\right\rangle .\left|\tilde{p}_{i}^{a}\right\rangle$ is the smooth projector functions 
satisfying the following orthogonality and completeness relations inside the augmentation spheres,

$$
\left\langle\tilde{p}_{i}^{a} \mid \tilde{\phi}_{j}^{a}\right\rangle=\delta_{i j}
$$

It makes sense to expand the smooth wavefunction into smooth partial waves,

$$
\left|\tilde{\psi}_{n}\right\rangle=\sum_{i} P_{n i}^{a}\left|\tilde{\phi}_{i}^{a}\right\rangle
$$

where $P_{n i}^{a}$ is the expansion coefficients. Multiplying both sides by $T$ gets,

$$
T\left|\tilde{\psi}_{n}\right\rangle=\sum_{i} P_{n i}^{a}\left|\phi_{i}^{a}\right\rangle
$$

By multiplying $\left\langle\tilde{p}_{i}^{a}\right|$ and using orthogonality and completeness relations,

$$
\left\langle\tilde{p}_{i}^{a} \mid \tilde{\psi}_{n}\right\rangle=\sum_{j} P_{n j}^{a}\left\langle\tilde{p}_{i}^{a} \mid \tilde{\phi}_{j}^{a}\right\rangle=\sum_{j} P_{n j}^{a} \delta_{i j}=P_{n i}^{a}
$$

we can rewrite wave functions in these forms:

$$
\begin{aligned}
\left|\tilde{\psi}_{n}\right\rangle & =\sum_{i}\left\langle\tilde{p}_{i}^{a} \mid \tilde{\psi}_{n}\right\rangle\left|\tilde{\phi}_{i}^{a}\right\rangle \\
\left|\psi_{n}\right\rangle & =\sum_{i}\left\langle\tilde{p}_{i}^{a} \mid \tilde{\psi}_{n}\right\rangle\left|\phi_{i}^{a}\right\rangle
\end{aligned}
$$

Thus the linear transformation operator is,

$$
T=1+\sum_{a} T^{a}=1+\sum_{a} \sum_{i}\left(\left|\phi_{i}^{a}\right\rangle-\left|\tilde{\phi}_{i}^{a}\right\rangle\right)\left\langle\tilde{p}_{i}^{a}\right| .
$$

Note that valence wavefunctions tend to have rapid oscillations near ion cores. This situation is problematic if one wants to describe the wavefunctions accurately. The goal of PAW approach is to transform these rapid oscillations wavefunctions into smooth wavefunctions. This is computationally very efficient and provides a way to calculate all-electron properties from these smooth wavefunctions. 


\subsubsection{Frozen core approximation}

In the many-electron system, one can either perform the calculations for all electrons, or focus only on the valence electrons and treat the core states separately. In the a frozen core approximation, atomic core states serve as basis function for the core wave function in the crystal, where

$$
\left|\psi_{n}\right\rangle=\left|\phi_{\alpha}^{a, \text { core }}\right\rangle
$$

for all core states $n$, here $n$ runs over $(a, \alpha)$, where $a$ is the atom index and $\alpha$ are the core states of an atom.

\subsubsection{Expectation values of local operators}

In the frozen core approximation expectation values can be obtained:

$$
\begin{aligned}
\langle O\rangle= & \sum_{n}^{\text {val }} f_{n}\left\langle\psi_{n}|O| \psi_{n}\right\rangle+\sum_{a} \sum_{\alpha}^{\text {core }}\left\langle\phi_{\alpha}^{a, \text { core }}|O| \phi_{\alpha}^{a, \text { core }}\right\rangle \\
= & \sum_{n}^{\text {val }} f_{n}\left\langle\tilde{\psi}_{n}|O| \tilde{\psi}_{n}\right\rangle+\sum_{a} \sum_{i, j}\left(\left\langle\phi_{i}^{a}|O| \phi_{j}^{a}\right\rangle-\left\langle\tilde{\phi}_{i}^{a}|O| \tilde{\phi}_{j}^{a}\right\rangle\right) D_{i j}^{a} \\
& +\sum_{a} \sum_{\alpha}^{\text {core }}\left\langle\phi_{\alpha}^{a, \text { core }}|O| \phi_{\alpha}^{a, \text { core }}\right\rangle
\end{aligned}
$$

where the tensor $D_{i j}^{a}$ is:

$$
D_{i j}^{a}=\sum_{n} f_{n}\left\langle\tilde{\psi}_{n} \mid \tilde{p}_{i}^{a}\right\rangle\left\langle\tilde{p}_{j}^{a} \mid \tilde{\psi}_{n}\right\rangle
$$

\subsubsection{Density}

Following this scheme, the electron density is given by,

$$
\begin{aligned}
n(\mathbf{r})= & \sum_{n} f_{n}\left|\psi_{n}(\mathbf{r})\right|^{2}=\sum_{n} f_{n}\left\langle\psi_{n} \mid \mathbf{r}\right\rangle\left\langle\mathbf{r} \mid \psi_{n}\right\rangle \\
= & \sum_{n}^{\text {val }} f_{n}\left|\tilde{\psi}_{n}(\mathbf{r})\right|^{2}+\sum_{a} \sum_{i, j}\left(\phi_{i}^{a}(\mathbf{r}) \phi_{j}^{a}(\mathbf{r})-\tilde{\phi}_{i}^{a}(\mathbf{r}) \tilde{\phi}_{j}^{a}(\mathbf{r})\right) D_{i j}^{a} \\
& +\sum_{a} \sum_{\alpha}^{\text {core }}\left|\phi_{\alpha}^{a, \text { core }}(\mathbf{r})\right|^{2}
\end{aligned}
$$


The functions $\phi_{\alpha}^{a, \text { core }}$ are not strictly localized withing the augmentation sphere.

\subsection{Ab-initio Molecular dynamics}

In contrast to Born-Oppenheimer molecular dynamics, which propagated nuclear degree of freedom by using ionic forces from solving the electronic problem, in 1985 Roberto Car and Michele Parrinello[72] have introduced the electronic degrees of freedom as fictitious dynamical variables. The fictitious dynamics relies on the fictitious electron mass to ensure adiabaticity. They showed that calculations with moving classical nuclei are feasable. The equations are derived as Euler Lagrange equations from the Lagrangian

$$
\begin{aligned}
\mathcal{L}= & \sum_{n} f_{n}\left\langle\dot{\psi}_{n}\left|m_{\psi}\right| \dot{\psi}_{n}\right\rangle+\frac{1}{2} \sum_{i} M_{i} \dot{\vec{R}}_{i}^{2}+E_{D F T}\left[\psi_{n}, \vec{R}_{i}\right] \\
& -\sum_{n, m} \Lambda_{n, m}\left(\left\langle\psi_{n} \mid \psi_{m}\right\rangle-\delta_{n, m}\right)
\end{aligned}
$$

The first term is the fictitious kinetic energy of the wave functions. The second term describes the classical kinetic energy of the nuclei. The third term is the density functional total energy, which is a functional of the wave functions and the atomic positions. The last term is the constraint of orthonormal wave functions $\left|\psi_{n}\right\rangle$. The matrix $\boldsymbol{\Lambda}$ are the corresponding Lagrange multipliers.

The Euler-Lagrange equations are

$$
\begin{aligned}
m_{\psi}\left|\ddot{\psi}_{n}\right\rangle f_{n} & =-\underbrace{\frac{\delta E}{\delta\left\langle\psi_{n}\right|}}_{\hat{H}\left|\psi_{n}\right\rangle f_{n}}+\sum_{m}\left|\psi_{m}\right\rangle \Lambda_{m, n} \\
M_{i} \ddot{\vec{R}}_{i} & =\underbrace{-\vec{\nabla}_{\vec{R}_{i}} E}_{\vec{F}_{i}}
\end{aligned}
$$

In the present work, we use the fictitious Lagrangian formalism.

\subsection{Calculation of spectra}

\subsubsection{ELNES and XANES}

ELNES describes that part of the electron-energy loss spectrum (EELS) which samples the contribution of a core-electron excitation of a given element to the empty orbitals. XANES is similarly based on the absorption of x-rays by excitations of core electrons into empty states. 
ELNES spectra describe the energy loss function

$$
F(\omega)=-\operatorname{Im}\left(\epsilon_{r}^{-1}(\omega)\right)
$$

due to the excitation of an core electron. $\epsilon_{r}(\omega)$ is the contribution of the corresponding processes to the relative dielectric constant and $\hbar \omega$ is the energy transferred from the fast probe electron. In this paper, we study the $K$-edge absorption of oxygen, where the $1 \mathrm{~s}$ core level is excited.

The relative dielectric constant is obtained as [73]

$$
\begin{aligned}
\epsilon_{r}(\omega)= & +\frac{4 \pi e^{2} N}{4 \pi \epsilon_{0} m_{e}} \\
& \times \sum_{n} \frac{Q_{n, 0}}{\left(E_{n}-E_{0}\right)^{2}-(\hbar \omega)^{2}-i \Gamma \hbar \omega}
\end{aligned}
$$

$E_{0}$ is the energy of the ground state and $E_{n}$ is the energy of the excited many-particle state with a core hole and the excited electron. In practice, the excitation energy $E_{n}-E_{0} \approx$ $\left(\epsilon_{n}-\epsilon_{c}\right)$ is determined as the difference between the energy $\epsilon_{n}$ of an unoccupied Kohn-Sham state $\left|\psi_{n}\right\rangle$ and the level $\epsilon_{c}$ of the core state $\left|\psi_{c}\right\rangle$. The oscillator strengths $Q_{n, 0}$ are obtained as

$$
Q_{n, 0}=\frac{2 m}{\hbar^{2}} \sum_{c}\left(\epsilon_{n}-\epsilon_{c}\right) \frac{1}{3} \sum_{j}\left|\left\langle\psi_{c}\left|x_{j}\right| \psi_{n}\right\rangle\right|^{2}
$$

The sum over states implicitly contains the spin multiplicity, i.e. every spin state is counted once. The sum over $c$ is the sum of core levels from the same shell. The index $c$ specifies the orbital momentum $m$ and the spin quantum numbers of the core shell. Only unoccupied band states contribute to the spectrum. $N$ is the number of oxygen atoms per unit volume. $\Gamma$ is the life-time broadening of the excitation.

In order to evaluate the matrix elements $\left\langle\psi_{c}\left|x_{j}\right| \psi_{n}\right\rangle$ between the core state $\left|\psi_{c}\right\rangle$ and the band state $\left|\psi_{n}\right\rangle$, we use the approximate representation of the wave function in terms of all-electron partial waves $\left|\phi_{n}\right\rangle$, i.e.

$$
\left|\psi_{n}\right\rangle \approx \sum_{n}\left|\phi_{\alpha}\right\rangle\left\langle\tilde{p}_{\alpha} \mid \tilde{\psi}_{n}\right\rangle
$$

When we express the dipole operator $x_{j}$ by cubic spherical harmonics, we can express the matrix elements using the Gaunt coefficients $C_{L, L^{\prime}, L^{\prime \prime}}=\int d \Omega Y_{L}^{*} Y_{L^{\prime}} Y_{L^{\prime \prime}}$ as

$$
\begin{aligned}
\left|\left\langle\psi_{c}\left|x_{j}\right| \psi_{n}\right\rangle\right|^{2}= & \mid \sum_{\alpha} \sqrt{\frac{4 \pi}{3}} C_{L_{c}, p_{j}, L_{\alpha}} \\
& \times\left.\int d r r^{3} R_{c}(r) R_{\alpha}(r)\left\langle\tilde{p}_{\alpha} \mid \tilde{\psi}_{n}\right\rangle\right|^{2}
\end{aligned}
$$


where $R_{\alpha}(r)$ is the radial part of the partial wave $\phi_{\alpha}(r)=R_{\alpha}(|r|) Y_{L_{\alpha}}(r)$, and $R_{c}(r)$ is the analogously defined radial part of the core level. The index $p_{j}$ species the cubic spherical harmonic with $\ell=1$, that points in the direction of $x_{j}$.

The life-time effects have been estimated as follows: The life-time broadening of the electron in the conduction bands has been estimated by Egerton [74] as

$$
\Gamma(\epsilon)=\frac{\hbar \sqrt{2 \epsilon / m_{e}}}{\lambda}
$$

by the free-electron velocity $\sqrt{2 \epsilon / m_{e}}$ and the inelastic mean free path $\lambda$. The energy $\epsilon$ is the energy of the band state relative to the absorption edge. For the inelastic mean free path we use the interpolating formula of Seah [75]

$$
\lambda=\frac{A}{\epsilon^{2}}+B \sqrt{\epsilon}
$$

with the values $A=641 \mathrm{eV}^{2} \mathrm{~nm}$ and $B=0.096 \mathrm{~nm} / \sqrt{\mathrm{eV}}$ for inorganic compounds from Seah's analysis.

The smaller effect is the lifetime of the core hole. We follow Egerton [74] and obtain the core-hole lifetime broadening for the K-shell from

$$
\Gamma_{K}=\left[-0.285+0.0216\left(\frac{E_{t h r}}{\mathrm{eV}}\right)^{0.472}\right] \mathrm{eV}
$$

where $E_{t h r}$ is the excitation energy at the absorption threshold. We use the calculated core-level energy $E_{t h r}=-515.3 \mathrm{eV}$, so that $\Gamma_{K}=0.70 \mathrm{eV}$.

In this paper, we use the so-called independent electron approximation (IPA), which ignores the presence of the core-hole. The only effect of the core hole considered is a rigid energy shift of the spectrum to adjust the absorption threshold to experiment. A more accurate calculation would use the same analysis, but for a supercell calculation with one-half of a core hole on the probed atom. The justification for the factor one-half is Slater's transition state rule. An even more advanced analysis requires the solution of the Bethe-Salpether equation [76].

The justification of the IPA in the present work is the limitation on the $\mathrm{O} K$ edge $(1 s-2 p$ transition) because it is particularly suited for an interpretation based on partial Density of States (DoS) calculations given the generally strong screening of the $1 s$ core hole [77]. In contrast, the spectral shape of the $\mathrm{Mn} L_{2,3}$ edge would be strongly affected by multiplet effects arising from the strong wavefunction overlap between the $2 p$ hole and the $3 d$ electrons.

\subsection{Computation codes}

The DFT calculations in the present work, are performed using the CP-PAW code, which contains the PAW method for the simulation of chemical binding and ab-initio molecular dynamics. 


\subsection{Summary}

The four approximations are discussed: LDA, GGA, Hybrid, and Local-hybrid. Since the aim of this thesis is to calculate electronic and structural properties of strongly correlated system, it is first necessary to ascertain what the best type of functional is to use. We use local hybrid functionals to account the Coulomb interaction on the transition-metal-sites and to obtain the physically correct description of the electronic structure. Emphasis is given to a realistic description of the material, and its impact on the reaction mechanism. 


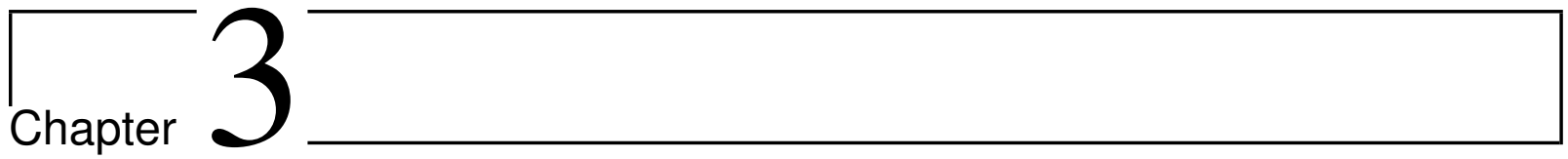

\section{Manganites}

This chapter is devoted to the study of a group of materials known as manganites. Manganites have attracted a lot of interest from the experimental and theoretical scientist due to their abnormal behavior upon the applied magnetic field. These materials are a new challenge to our understanding as a strongly correlated electron systems. There are several important reasons that motivate everyone to study this important area such as unexpectedly large magnetotransport properties, rich phase diagram and strong correlations between charge, spin, orbital, and lattice degrees of freedom. Here we try to analyze and investigate the electronic structure of $\mathrm{Pr}_{1-x} \mathrm{Ca}_{x} \mathrm{MnO}_{3}$ and $\mathrm{La}_{1-x} \mathrm{Sr}_{x} \mathrm{MnO}_{3}$ compounds and comparison them with experimental data using first-principles calculations. The effect of various ordering phenomena for ferromagnetic (B-type) and selected antiferromagnetic (A-, C-, and G-type) arrangements, including band gap and magnetic moment are investigated. Then, calculated spectra with x-ray photoelectron spectroscopy (XPS), electron energy loss near edge structure (ELNES) are discussed. The summary is the last part of this chapter.

\subsection{Importance of manganites}

From a historical point of view, the term "manganites" came from the paper in 1950 by Jonker and Van Santen [78]. In their report La has been replaced either by $\mathrm{Ca}, \mathrm{Sr}$, and $\mathrm{Ba}$ which create $(\mathrm{La}, \mathrm{Ca}) \mathrm{MnO}_{3}$, (La,Sr) $\mathrm{MnO}_{3}$, and (La,Ba) $\mathrm{MnO}_{3}$ samples. They found the appearance of ferromagnetism in these compounds. The term manganites refer to the compounds containing trivalent or tetravalent Mn atoms. Few months later, by these authors, the conductivity of manganites [79] and the effect of hole doping on the lattice parameter [80] have been discussed. From their observation, the crystal of $\mathrm{LaMnO}_{3}$ is distorted while there is no distortion at higher $\mathrm{Sr}$, and $\mathrm{Ca}$ doping. These distortions are attributed to the very important concept in manganites which is so-called Jahn-Teller (JT) effect.

In the early investigation of manganites, Wollan and Koehler [81] specified the magnetic 
structure of $\mathrm{La}_{1-x} \mathrm{Ca}_{x} \mathrm{MnO}_{3}$ in the entire range of doping by using neutron diffraction techniques. They found other interesting antiferromagnetic phases and also evidence of charge ordering phase in manganites.

Another popular type of manganites, $\mathrm{Pr}_{1-x} \mathrm{Ca}_{x} \mathrm{MnO}_{3}$, was studied by Jirak et al. [4]. They found phases with charge ordering which is totally different from the ferromagnetic metallic phases of other manganites. In addition to the charge ordering phase, a deviation from cubic structure, different magnetic structures and distortions were reported. At small hole doping, $x \sim 0$, A-type (ferro in plane and antiferro between planes) antiferromagnetic order with orthorhombic structure was found while for large hole doping, $x \sim 1$, G-type (completely antiferro) antiferromagnetic order with less distorted structure was obtained. These distortions are attributed to the Jahn-Teller effect and also ionic size of $\mathrm{Pr}$ and $\mathrm{Ca}$ which is similar to each other.

During the 1990s, experimental work on manganites attracted wide attention due to the observation of large magnetoresistance (MR) effects in these compounds. Chahara et al. [82] found large MR for thin films of $\mathrm{La}_{1-x} \mathrm{Ca}_{x} \mathrm{MnO}_{3}$ at $x=0.25$ and Ju et al. [83] also observed this effect in the films of $\mathrm{La}_{1-x} \mathrm{Sr}_{x} \mathrm{MnO}_{3}$ near room temperature. Jin et al. [84] reported a colossal MR ratios in study of $\mathrm{La}_{0.67} \mathrm{Ca}_{0.33} \mathrm{MnO}_{x}$ films which was higher than previously studies. From these important results, it is clear that more experimental and theoretical studies are needed to explore and explain the colossal MR.

Despite of the many materials such as metals which can be defined as a free- and independent-electron system, manganites are well known as a strongly correlated systems, as they show a large variety of different structural, magnetic and electronic phases. In these compounds, interactions between the $d$-electrons are not weak, and these interactions play a major role in determining the properties of such systems. In correlated materials, charge, spin, orbital and lattice degrees of freedom result in competing interactions. These lead to phase transitions and the emergence of exotic phases including charge-ordered [85], multiferroic [5] and pseudogap states [86]. In, $\operatorname{Pr}_{1-x} \mathrm{Ca}_{x} \mathrm{MnO}_{3}$, the material studied in this investigation, the number of electrons in the system can be varied systematically without greatly affecting the crystal structure and all compositions from $\mathrm{PrMnO}_{3}$ to $\mathrm{CaMnO}_{3}$ can be synthesized [5, 36, 87]. Another characteristics on the doped compounds $\left(\mathrm{CaMnO}_{3}\right)$ arises from their potential benefit as photocatalysts [13], inspired by the use of a $\mathrm{CaMnO}_{x}$ complex as oxygen-evolution center in natural photosynthesis [14].

In next section, the crystal structures of $\operatorname{Pr}_{1-x} \mathrm{Ca}_{x} \mathrm{MnO}_{3}$ will be discussed.

\subsection{Perovskite lattice}

\subsubsection{Ideal structure}

Manganites crystallize in a perovskite structure as shown in Fig. 3.1. This structure is an inorganic compound with the formula $\mathrm{ABO}_{3}$, consisting of oxygen octahedra, where $\mathrm{B}$ is the atom in the center of the octahedron, i.e. $\mathrm{Mn}^{3+}$ and $\mathrm{Mn}^{4+}$ ions, and $\mathrm{A}$ is the cation 
outside the octahedron such as $\mathrm{Pr}^{3+}, \mathrm{Ca}^{2+}, \mathrm{La}^{3+}$, and $\mathrm{Sr}^{2+}$. The spacegroup for cubic perovskites is $\operatorname{Pm} \overline{3} m$ (221); the equivalent positions of the atoms are detailed in Tab. 3.1.

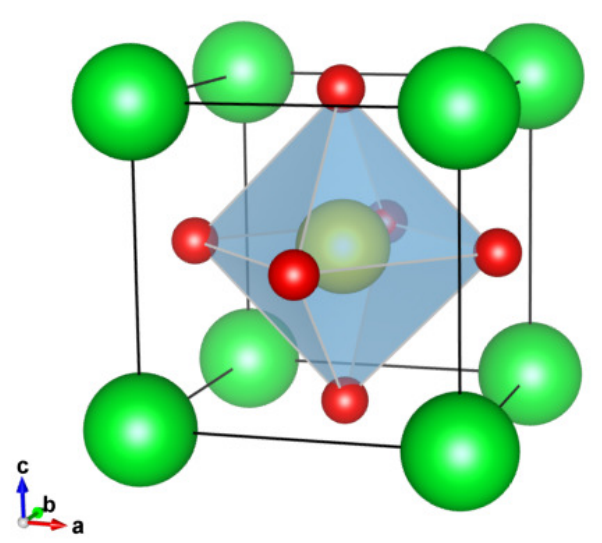

Fig. 3.1: Cubic perovskite unit cell. Red spheres represent the oxygen anions, green spheres represent the A cations, and yellow one shows the B cation.

Table 3.1: Atomic positions for ideal perovskite structure in Cartesian coordinate.

\begin{tabular}{ccc}
\hline \hline Site & Location & Co-ordinates \\
\hline A cation & $(2 \mathrm{a})$ & $(0,0,0)$ \\
B cation & $(2 \mathrm{a})$ & $\left(\frac{1}{2}, \frac{1}{2}, \frac{1}{2}\right)$ \\
O anion & $(6 \mathrm{~b})$ & $\left(\frac{1}{2}, \frac{1}{2}, 0\right)\left(\frac{1}{2}, 0, \frac{1}{2}\right)\left(0, \frac{1}{2}, \frac{1}{2}\right)$ \\
\hline \hline
\end{tabular}

The members of the perovskite family rarely have the ideal cubic structure. In fact there is a large amount of structural distortions and electronic instabilities. In the case of manganites, $\operatorname{Pr}_{1-x} \mathrm{Ca}_{x} \mathrm{MnO}_{3}$, the cubic perovskite structure is distorted into an orthorhombic structure by a tilting of the oxygen octahedra.

\subsubsection{Octahedral tilting}

If the A-type ions of the perovskite $\mathrm{ABO}_{3}$ are too small, octahedra tilts and allows to increase the atom density and therefore improve the Coulomb attraction energy for the ions. The octahedral tilting is determined by the so-called tolerance factor [88] which is defined as: 


$$
t=\frac{d_{A O}}{\sqrt{2} d_{B O}}
$$

where $d_{A O}$ is the distance between the A-site atoms (La, Ca, Pr) and oxygen, while $d_{B O}$ is the B-site ions (Mn) and oxygen distance. This parameter for cubic peovskite is about $0.9<t<1.0$. However, if the radius of the A-site cation is too small $(0.71<t<0.90)$, the oxygen octahedra tends to undergo rigid tilts [89]. The distortions seen in $\mathrm{CaMnO}_{3}$ are characteristic of this effect which makes orthorhombic structure. On the other hand, compositions with $t>1$ will exhibit hexagonal symmetry [89].

The orthorhombic phase is the most common structure of manganites. Thus, octahedral tilting is an important ingredient. The octahedral tilting in $\mathrm{ABO}_{3}$ compositions can be characterized by the so-called Glazer notation [90]. In this notation, the octahedral tilting can be described by using symbols $a^{*} b^{*} c^{*}$, which the letters refer to tilt around the [100], [010], and [001] directions of the ideal cubic perovskite. The superscript $*$ denotes the octahedral tilts which can be in the same $(+)$ or opposite $(-)$ and no tilt $(0)$. The orthorhombic structure of $\mathrm{ABO}_{3}$ can be classified by $a^{-} a^{-} c^{+}$tilting which is shown in Fig. 3.2. This $a^{-} a^{-} c^{+}$tilting basically originated from the smaller size of A-site atoms. When A-site atoms are larger, for example $\mathrm{SrMnO}_{3}$, the tilting does not occur. So, the $\mathrm{SrMnO}_{3}$ belongs to the ideal cubic perovskite which is also shown in Fig. 3.2 . The physical properties such as band gap, density of states and effective carrier mass are closely related with the amount of tilting. Note that the overlapping integral of the $\mathrm{BO}_{6}$ octahedron is one of the main sources of various electronic properties.

(a)

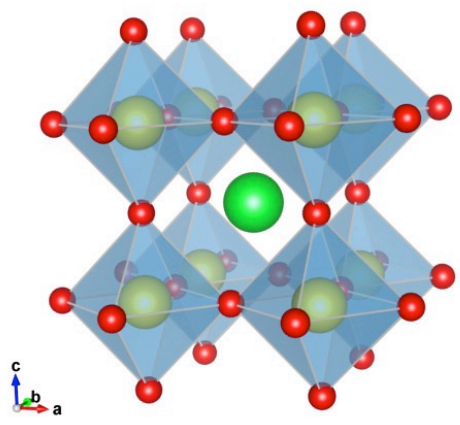

no tilt (b)

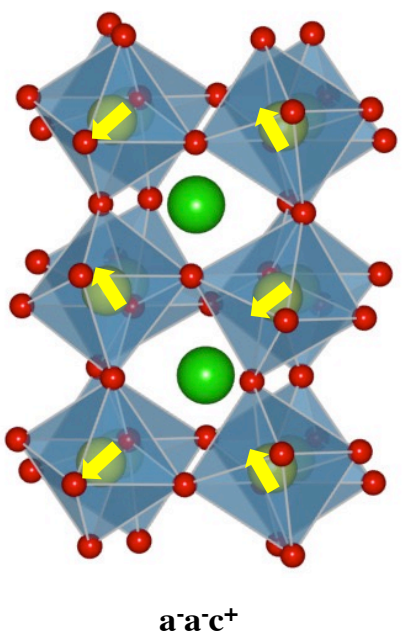

Fig. 3.2: The ideal perovskite structure and perovskite with octahedral tilting, (a) $P m \overline{3} m$ (221), and (b) Pnma (62) orthorhombic with $a^{-} a^{-} c^{+}$tilting. Arrows show the tilting directions. 


\subsection{Energy levels}

\subsubsection{Crystal field splitting}

Let us start our discussion at the level of atomic problem. The $d$-orbital energies of a transition-metal $(\mathrm{Mn})$ ion will be affected by an octahedral arrangement of six oxygen atoms. The main interactions for forming the electronic structure and for magnetism are through the $\mathrm{O}-2 p$ orbitals which mix with the transition metal $d$-orbitals. The Pauling electronegativity of $\mathrm{O}$ is higher than any transition element. Therefore, the transition metal $d$-band lies above the $\mathrm{O}-p$ band. In a cubic environment, the five $d$-orbitals are separated by symmetry into a two-fold degenerate $e_{g}$ and a three-fold degenerate $t_{2 g}$ sets. The splitting between the $e_{g}$ and $t_{2 g}$ is commonly denoted $10 D_{q}$ [3]. The ligand field splitting of the pure $d$ orbitals for the tetrahedral $T_{d}$, octahedral $O_{h}$ and tetragonal $D_{4 h}$ symmetries are shown in Fig. 3.3.
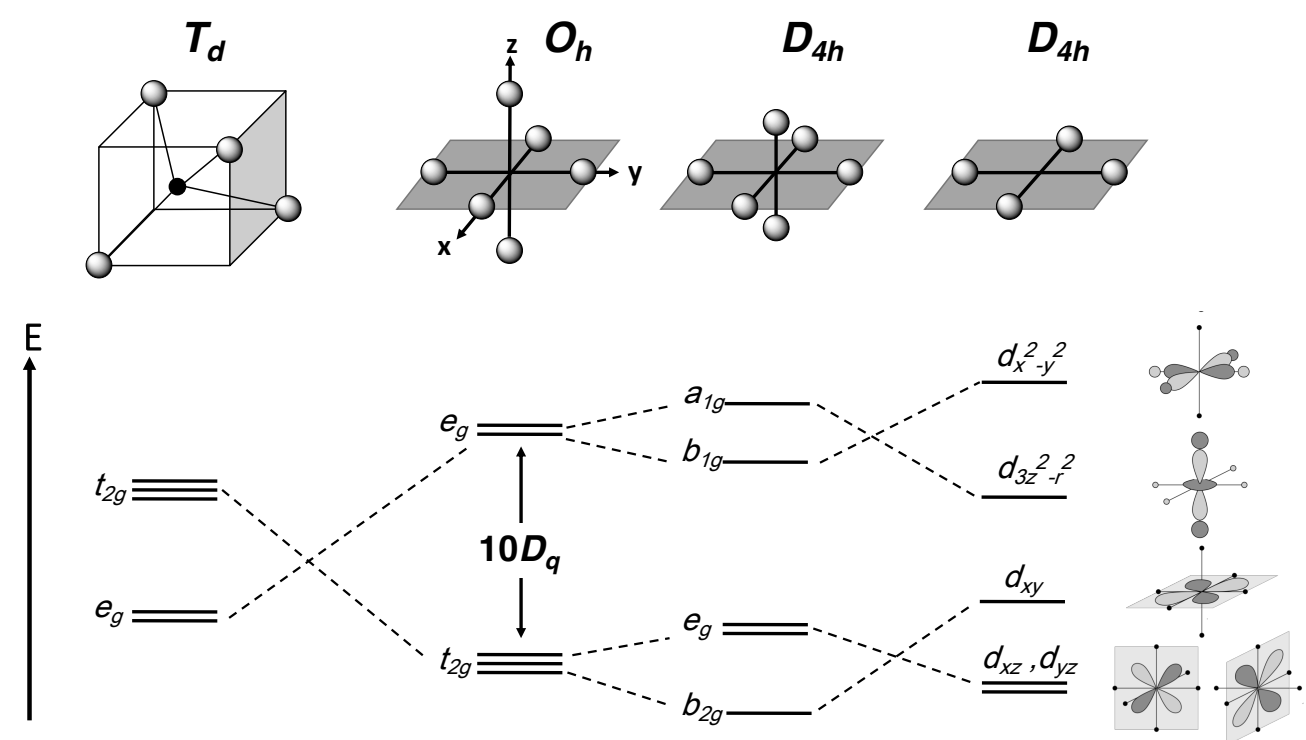

Fig. 3.3: Schematic representation of the crystal field splitting of the $d$-levels in fields of different coordination and symmetry. The central Mn- $d$ ion is assumed to be coordinated by oxygen atoms. $T_{d}$ corresponds to a tetrahedral environment and $O_{h}$ corresponds to a cubic symmetries. For octahedral $O_{h}$ and tetragonal $D_{4 h}$ symmetries, equal strengths in the $x-y$ are assumed while the strengths along $z$ are varied. On the right relevant orbitals corresponding to $e_{g}$ symmetry and $t_{2 g}$ symmetry are indicated. Different sign of $d$ wavefunction lobes are shown with different color.

The $e_{g}$ orbitals, labeled $d_{x^{2}-y^{2}}$ and $d_{3 z^{2}-r^{2}}$, have lobes that point towards the $\mathrm{O}$ ions in an octahedral environment, while the $t_{2 g}$ orbitals, $d_{x y}, d_{x z}$ and $d_{z y}$ point in between the $\mathrm{O}$ ions. As shown in Fig. 3.4, there is a strong $\sigma$-bonding interaction between the O- $p$ and the 
$e_{g}$-orbitals and a weaker $\pi$-bonding between the $\mathrm{O}-p$ orbitals and the $t_{2 g}$-orbitals. In other words, the $t_{2 g}$-bands are more weakly antibonding than the $e_{g}$ bands, and therefore occur lower in energy. This is shown in Fig. 3.4. The crystal field scheme in perovskites originates from covalent interaction with the oxygen neighbors. It is not electrostatic origin [91]. Furthermore, it can be seen that in perovskites, the $t_{2 g}$-bands are generally narrower than the corresponding $e_{g}$-bands. The $e_{g}$-bands have a more hybridization character than the $t_{2 g}$-bands. Importantly, large crystal field splittings are an indicator of strong hybridization. In practice, typical distances for splitting are between 1 and $2 \mathrm{eV}$.

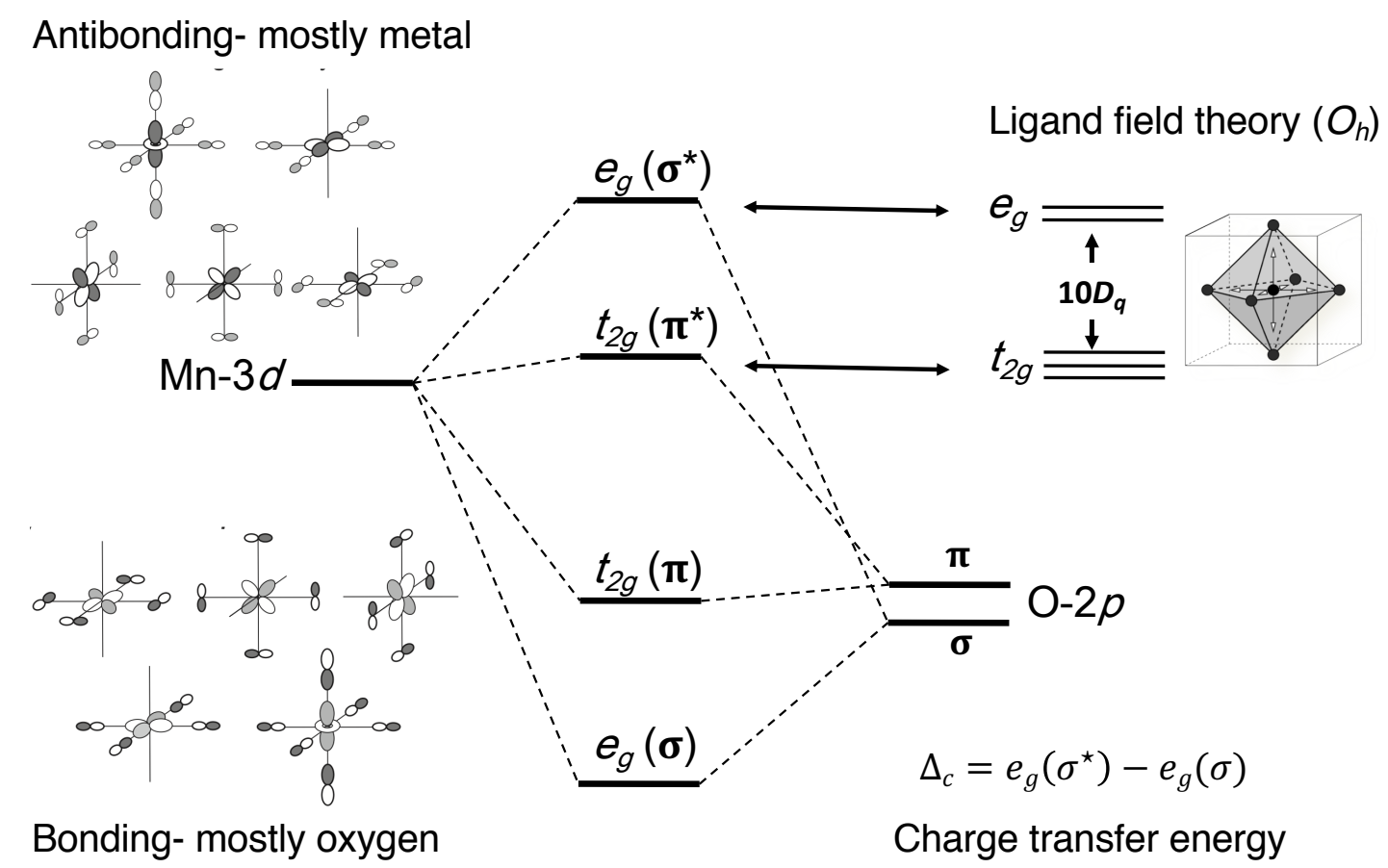

Fig. 3.4: Demonstration of molecular orbital theory for a Mn atom, surrounded by six oxygen atoms in an octahedral environment. The relevant system is shown in the top right. Bonding and antibonding orbitals according to symmetry class are shown in the left. The bonding is caused by orbital overlap of the Mn- $d$ orbitals with O- $2 p$ orbitals. The bonding orbitals have mostly oxygen character while the antibonding orbitals have mostly metal-ion orbitals. The correspondence between the $e_{g}^{*}$ and $t_{2 g}^{*}$ molecular orbitals and the $e_{g}$ and $t_{2 g}$ orbitals in a crystal field picture is shown in the top right. In the lower right the charge transfer energy $\Delta_{c}$ is defined as the average energy required to excite an electron from the $\sigma$ bonding to the $\sigma^{*}$ antibonding state.

\subsubsection{Jahn-Teller distortion}

The splitting due to crystal-field effects leads to an $e_{g}$ doublet and a $t_{2 g}$ triplet. The remaining degeneracy is usually broken by the lattice distortion. The lifting of degeneracy 
due to the orbital-lattice interaction is called the Jahn-Teller (JT) cooperative effect. The effect is named for Hermann Arthur Jahn and Edward Teller, who first reported studies about it in 1937 [92].

The JT distortion is obtained when the $e_{g}$-orbitals are partially occupied. The $e_{g^{-}}$ orbitals are the $d$-electrons pointing towards the oxygen neighbors. The elongation of two axial bonds weakens the antibonds and thus lowers one of the two $e_{g}$-orbitals. The JT distortions are due to an different bond length for the three axis. In the case of manganites, $\mathrm{Pr}_{1-x} \mathrm{Ca}_{x} \mathrm{MnO}_{3}$, when a Ca ion is replaced by a $\mathrm{Pr}$ ion, a $\mathrm{Mn}^{4+}$ ion becomes a $\mathrm{Mn}^{3+}$ ion to maintain charge neutrality. The electrons in $\mathrm{Mn}^{3+}$ are in the state of maximum spin $S=2$ due to the strong Hund coupling. It is equivalent to having the extra electron in the empty $e_{g}$-orbital of the $\mathrm{Mn}$ ion. For the $\mathrm{Mn}^{3+}$ ion, the electron localizes in the $d_{3 z^{2}-r^{2}}$ orbital which is energetically favorable if the octahedron distorts along the $z$ axis and so the oxygen ions in this direction move away from the orbital lobes. If the octahedron compresses along the $z$ axis, the electron localizes in the $d_{x^{2}-y^{2}}$ orbital. These two modes are shown in Fig. 3.5. For our compounds, only $Q_{2}=\frac{1}{\sqrt{2}}\left(d_{x}-d_{y}\right)$, and $Q_{3}=\frac{1}{\sqrt{6}}\left(-d_{x}-d_{y}+2 d_{z}\right)$ are needed to understand how the $e_{g}$ degeneracy is removed. $d_{x}, d_{y}$, and $d_{z}$ are displacement of oxygen atoms in the $x, y$, and $z$-direction respectively.

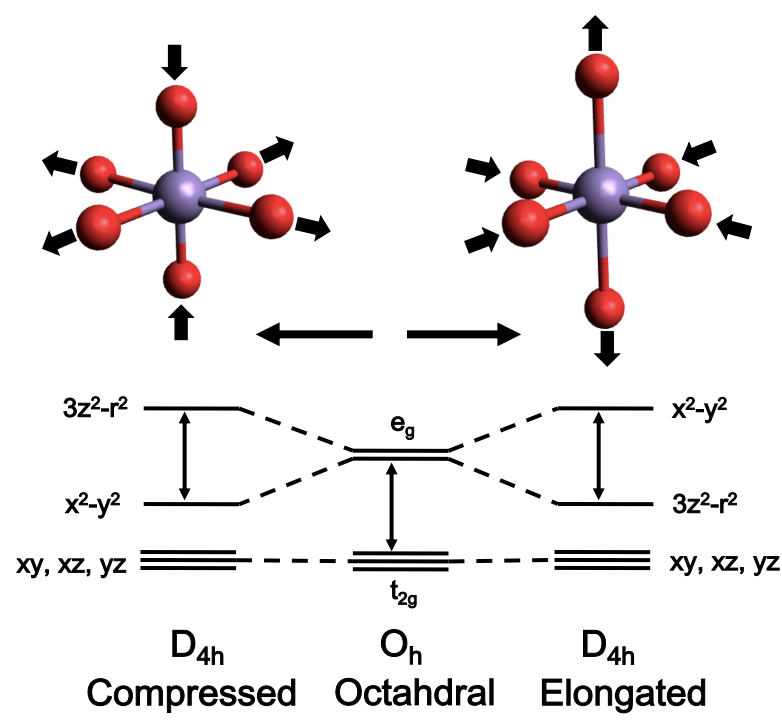

Fig. 3.5: Effect of a Jahn-Teller distortion on $d$-orbitals. The $\mathrm{Mn}^{3+}$ ion (violet) is located in the center of an oxygen (red) octahedron. When the octahedral symmetry is distorted, the energy of the $e_{g}$ orbitals which point along the elongated bond direction, is lowered in energy. Since the $t_{2 g}$ states are filled and they have a non-bonding character, they do not contribute to the splitting.

We can draw the Jahn-Teller distortions in a polar coordinates using $Q_{3}=Q \cos \theta$, $Q_{2}=Q \sin \theta$ and $Q=Q_{2} \sin \theta+Q_{3} \cos \theta$. Following Kanamori [93], if $\theta=0$, the distortion is purely of the $Q_{3}$ mode and it shows an elongation of the octahedra along the $z$-axis and contraction along the other axis. If $\theta=\pi$, it is also a pure $Q_{3}$ but with the reversed 
elongation and contraction. In the case $\theta=\frac{2 \pi}{3}$ the same distortion but with a different axis are produced. This information summarized in Fig. 3.5.

\subsubsection{Breathing}

The breathing parameter is given by the average bond length of all ligand bonds, which is called $Q_{1}$ mode. In other words, it is an isotropic expansion of octahedra. This parameter can be expressed by $Q_{1}=\frac{1}{\sqrt{3}}\left(d_{x}+d_{y}+d_{z}\right)$. It should be noted that the $Q_{1}$ mode does not contribute to the splitting in the $e_{g}$ states, but in the electronic structure, it is related to energy level shifts of the conduction electrons and thus constitutes of the electron-phonon coupling.

\subsection{Magnetic order}

Vast variety of magnetic structure is one of the feature of manganite compounds. By using neutron diffraction techniques to characterize the magnetic structure, Wollan and Koehler [81] found that, in addition to ferromagnetic (FM) phase there are many other antiferromagnetic (AFM) phases in manganites. A sketch of the magnetic arrangements is presented in Fig. 3.6.
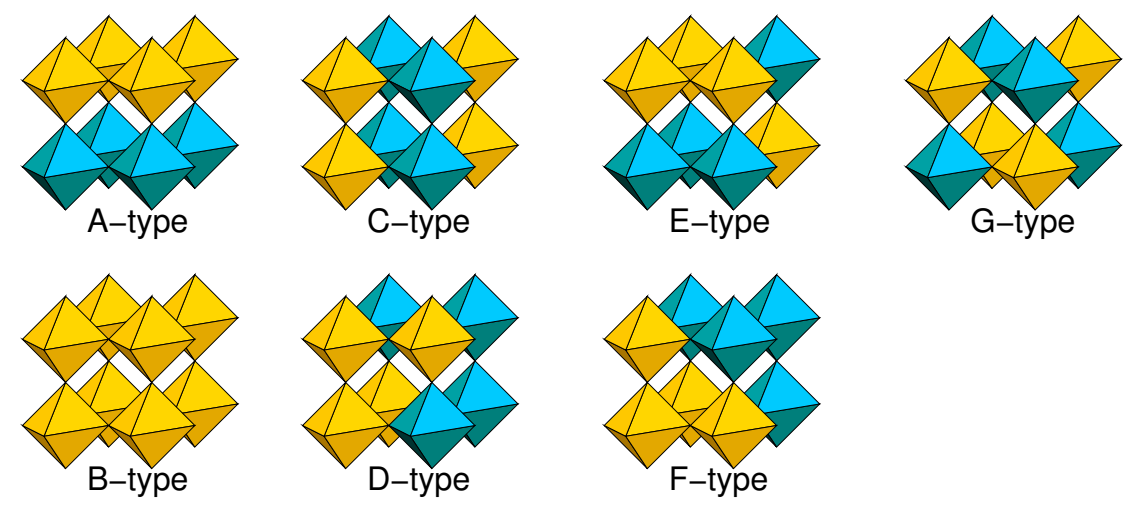

Fig. 3.6: Magnetic configurations for orthorhombic structure which yellow octahedron stands for the spin up and blue one shows spin down component. It is given from Wollan and Koehler [81]. A-type has ferromagnetic planes that are coupled antiferromagnetically. The B-type structure is the ferromagnetic arrangement, while G-type shows and antiferromagnet in all three directions. The C-type has ferromagnetic chains that are coupled antiferromagtenically. Figures are taken from $\Phi$ SX lecture notes, with kind approval of $\mathrm{P}$. Blöchl.

Manganites either have ordered electron spins or these spins are not ordered unless an external field induces some non-random orientation. These compounds exhibit FM, AFM and paramagnetic properties, which depend upon the relative trivalent and tetravalent manganese ion content and temperature. They are including different form of AFM 
arrangements. They differ in the relative ordering of their spins within the lattice. In the present study, manganites in orthorhombic cell for A-, C-, G-type AF and B-type FM configurations have been considered. As shown in Fig. 3.6, pattern A corresponds to planes with ferromagnetic alignment of spins, but with antiferromagnetic coupling between those planes. The G-type was found with antiferromagnetism in all three directions. In the C-type pattern, spins are ferromagnetic along the chains, but antiferromagnetic in the coupling chains. In the ferromagnetic state, the pattern was called B. The most exotic spin arrangement is the CE-type state. In the CE-type magnetic structure, the material exhibits one-dimensional zig-zag chains of ferromagnetically coupled Mn ions. This phase is the nontrivial phase which will be discussed later in more details.

In next section, the experimental phase diagram of $\operatorname{Pr}_{1-x} \mathrm{Ca}_{x} \mathrm{MnO}_{3}$ will be discussed. This includes structural and magnetic transition temperatures in that compound.

\subsection{The $\operatorname{Pr}_{1-x} \mathrm{Ca}_{x} \mathrm{MnO}_{3}$ Phase Diagram}

$\operatorname{Pr}_{1-x} \mathrm{Ca}_{x} \mathrm{MnO}_{3}$ is a mixed valent system due to the substitution of $\operatorname{Pr}^{3+}$ ions by $\mathrm{Ca}^{2+}$ which produces the rich variety of phases. At low temperature, there is no stable ferromagnetic metallic phase as shown in Fig. 3.7. This is usually rationalized by the fact that $\mathrm{Ca}$ and $\mathrm{Pr}$ ions are too small to fill the space in the cube centers and for this reason the oxygens tend to move toward that center. The Mn-O-Mn angle gets smaller than $180^{\circ}$ and the hopping amplitude for carriers to move from $\mathrm{Mn}$ to $\mathrm{Mn}$ naturally decreases as angle reduced from $180^{\circ}$. It should be noted that ferromagnetism is observed, but with insulating behavior in the resistivity, creating a FM insulator (FMI) phase.

This compound was first studied in the full range of Ca concentrations $0<x<1$ by Jirak et al. [4]. These authors measured the neutron diffraction pattern and electrical measurements over a wide temperature range. They showed with varying Ca concentrations, substantial changes in the magnetic interactions are introduced. As a consequence, a variety of magnetic structures are observed at low temperatures. Fig. 3.7 illustrates the many phases that are in competition.

At low temperatures, the general phase diagram evolves from an insulating antiferromagnet (AF) in the $0<x<0.12$, to a FMI phase in the $0.12<x<0.30$ region [3]. Around $x=0.5$ concentration, charge, spin, and orbital ordering are observed and, at $x=1$ the system goes back to an AF insulating phase [3]. The room-temperature Pbnm phase of $\operatorname{Pr}_{1 / 2} \mathrm{Ca}_{1 / 2} \mathrm{MnO}_{3}$ undergoes a charge-ordering $(\mathrm{CO})$ transition around $T_{C O} \sim 240$ $\mathrm{K}$ accompanied by an orbital and spin ordering of CE-type below $T_{N} \sim 180[4,6,94]$. The COI phase has been refined by selected area electron diffraction as $P 2_{1} m n$ characterised by a much wider dispersion of Mn-O-Mn bonding angles and $\mathrm{Mn}-\mathrm{O}$ bonding lengths (1.87 - $2.04 \AA$ ) [41]. The paramagnetic insulating phase (PI) arises at room temperature for concentrations of less than 0.3 and a charge-ordered insulating (COI) states is stabilized for $x \geq 0.3[3]$.

As Jirak et al. showed [4], $\mathrm{Pr}_{1-x} \mathrm{Ca}_{x} \mathrm{MnO}_{3}$ shows stable CO-state in the wide range 


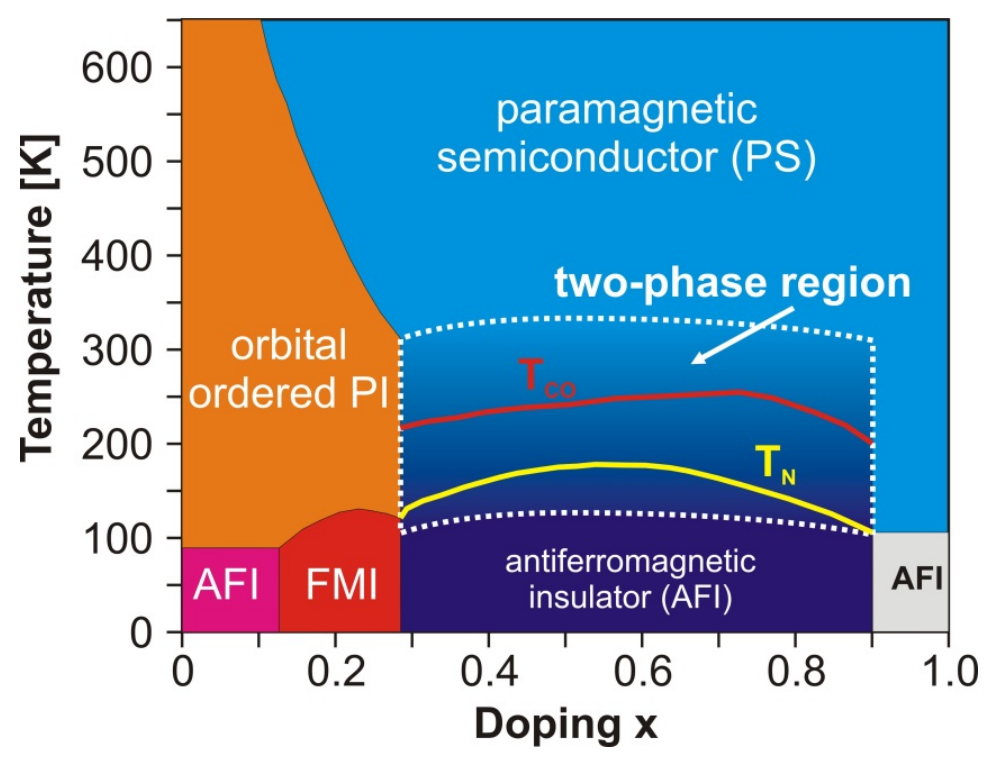

Fig. 3.7: The phase diagram of $\mathrm{Pr}_{1-x} \mathrm{Ca}_{x} \mathrm{MnO}_{3}$ from Christian Jooss. PI, FMI, and AFI denote paramagnetic insulator, ferromagnetic insulator, and antiferromagnetic insulator, respectively. $T_{C O}$ and $T_{N}$ indicate the temperature for charge-ordering transition and Néel temperature, respectively. With kind approval of C. Jooss.

of the Ca concentrations, between $x=0.3$ and 0.9 that the arrangement of charge, spin, and orbital order for these concentrations are similar to the CE-state. For $x=\frac{3}{8}$ based on mean-field and numerical approximations, Hotta and Dagotto [95] have offered and suggested that this FMI may present CO. Alterations from AF at $x=0.5$ to FM at $x=0.3$ have studied by neutron diffraction [96], and a canted state has also been proposed to model this behavior. Recent neutron diffraction data by Daoud-Aladine et al. [40] obtained the formation and ordering of Zener polaron (ZP) in $\operatorname{Pr}_{0.6} \mathrm{Ca}_{0.4} \mathrm{MnO}_{3}$, while Grenier et al. [39] supports a model involving orbital ordering (checkerboard) structure for $\mathrm{Pr}_{0.6} \mathrm{Ca}_{0.4} \mathrm{MnO}_{3}$. Ab-initio [97] and unrestricted Hartree-Fock [98] calculations reveal that in certain circumstances the ZP-ordered state in half-doped manganites may be desirable. Theoretical paper [99] shows that lowest energy ZP- and checkerboard-type ordered states may differ only by slight displacements of key oxygen ions. Both types of structures have been discussed by Efremov et al. [42] considering the site-centered charge ordering at $\mathrm{Mn}$ sites or bond-centered charge ordering at Mn-O-Mn dimers, forming the ZP-ordered state. Based on ab-initio calculation, Collizi et al. [100] reported a unconventional multiferroism in $\mathrm{Pr}_{1 / 2} \mathrm{Ca}_{1 / 2} \mathrm{MnO}_{3}$. Proper knowledge of the electronic structure around the Fermi level $\left(\mathrm{E}_{F}\right)$, governing both transport properties and low-energy excitations, is essential to take advantage of the material's susceptibility to diverse external stimuli and its flexibility in terms of mixed Mn valencies.

As already explained, low-bandwidth compounds, $\operatorname{Pr}_{1-x} \mathrm{Ca}_{x} \mathrm{MnO}_{3}$, in the vicinity of $x=0.5$ a charge-order phase is more stable, while in intermediate band-width compounds such as $\mathrm{La}_{1-x} \mathrm{Ca}_{x} \mathrm{MnO}_{3}$ have shown metallic phase at this hole density. The prevailing 
theories used to explain these two ground states called double exchange and superexchange which are discussed next.

\subsubsection{Double exchange and the FM phase}

The double exchange mechanism was suggested in 1951 by Zener in a couple of seminal papers [101, 102], to explain the ferromagnetic metallic phase at low temperatures. As shown in Fig. 3.8, electrons are thought to hop from one Mn-ion to the next via the intervening oxygen atom. The Hund's rule coupling, $J_{H}$, is enforced on each individual ion and makes it favorable for the spin of the core electrons to align with the spin of the itinerant electron and thus this electron transfer scheme makes ferromagnetic alignment energetically favorable. In other words, the conduction electrons lower their kinetic energy if the $t_{2 g}$ spins of manganites, are fully polarized. The kinetic energy is regulated by a hopping amplitude usually denoted by $t$. The coupling involved in the direct virtual hopping of $d$-electrons not mediated by conduction electrons, is called $J_{A F}$.

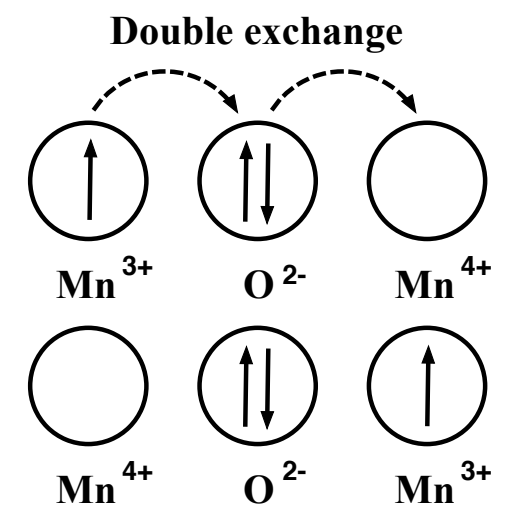

(a)

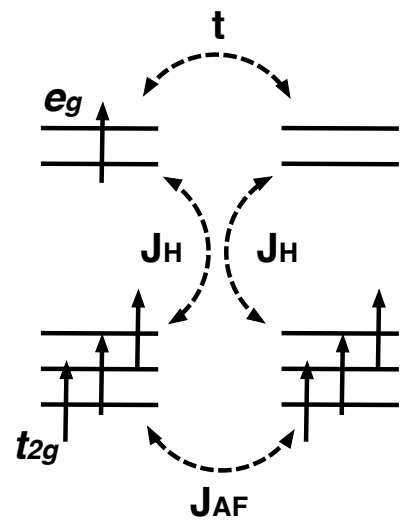

(b)

Fig. 3.8: Schematic representation of the idea of Zener ferromagnetism: (a) double exchange mechanism, (b) mobility of $e_{g}$ electrons. The couplings of relevance are the Hund coupling, $J_{H}$, the hopping $t$, and the antiferromagnetic direct exchange $J_{A F}$.

Anderson and Hasegawa [103] studied the Zener mechanism in greater detail. They explained the probability of transferring an electron from one Mn-ion to the next as a function of the angle which one core spin makes with its neighbor. In this theory, the electrons in the Mn-3d levels treat either as localized core electrons on each Mn-site or as itinerant electrons as shown in Fig 3.8. The core spins are treated classically, which means that they have a well defined direction. So, the total core spins for Mn-ion is $S=\frac{3}{2}$ and can lie at any angle with respect to its neighbor. The itinerant electron is treated quantum mechanically, and its spin can only be up or down. It should be noted that the oxygen 
between the Mn-ions has a passive role in the conduction process. Thus, its effect on the conductivity is absorbed into the proportionality constant.

This double exchange theory predicts that the ferromagnetic phase has the core spins of every Mn-ion aligned parallel and so has the best conductivity. The antiferromagnetic phase has the core spins aligned antiparallel and so has the worst conductivity and the paramagnetic phase has an intermediate conductivity.

\subsubsection{Superexchange and the AF Charge Ordered Phase}

This section contains (1) a description of superexchange and (2) the Goodenough-Kanamori rules. The latter combine double and superexchange.

In spite of the double exchange interaction where a ferromagnetic state was produced by the real exchange of an electron, the magnetic interactions in the charge ordered state arise from a virtual electron exchange process known as superexchange. The charge ordered phase is found to be antiferromagnetic and such an antiferromagnetic state can be formed by considering the interactions of the oxygen and neighboring Mn-ions. In this case, O-ion electrons affect the magnetic state of the neighboring Mn-ions. Several types of magnetic interaction can take place depending on the electron shells of the Mn-ions that summarized by the Goodenough-Kanamori rules [85] as below:

- If empty Mn-orbitals point towards neighboring empty Mn-orbitals, the exchange interaction is antiferromagnetic.

- If half-full Mn-orbitals point towards neighboring half-full Mn-orbitals, the exchange interaction is antiferromagnetic.

- If empty Mn-orbitals point towards neighboring half-full Mn-orbitals, the exchange interaction is ferromagnetic.

\subsubsection{Discussion of Double Exchange and Superexchange}

The theories of double exchange and superexchange are used to explain the magnetic properties and conductivity of manganites. However, one of the main problems with these theories is that they can not predict the transition from one manganite to another by doping concentration. On the other hand, $\operatorname{Pr}_{1-x} \mathrm{Ca}_{x} \mathrm{MnO}_{3}$ has no ferromagnetic metallic phase and for $x>0.3$ is a charge ordered antiferromagnet. Furthermore, the paramagnetic state of $\mathrm{La}_{1-x} \mathrm{Sr}_{x} \mathrm{MnO}_{3}$ is metallic. Therefore the structure plays a dominant role in determining the electrical and magnetic properties of the manganites. The typical explanation for the fact that $\operatorname{Pr}_{1-x} \mathrm{Ca}_{x} \mathrm{MnO}_{3}$ has no ferromagnetic metallic state is that the structure is more distorted than $\mathrm{La}_{1-x} \mathrm{Sr}_{x} \mathrm{MnO}_{3}$. Thus, the metallic state is observed to be more cubic than the insulating state. It is thought that $\operatorname{Pr}_{1-x} \mathrm{Ca}_{x} \mathrm{MnO}_{3}$ cannot obtain the structure for metallicity and thus its ground state is antiferromagnetic and insulating. In addition, all 
manganites show the charge ordered antiferromagnetic state for $x>0.5$ which the current theories offer no explanation of why this should be.

It is seen that a charge separation into $\mathrm{Mn}^{3+}$ and $\mathrm{Mn}^{4+}$ can not literally take place [40]. So, the theory of charge and orbital ordering does not seem entirely satisfactory. For example, the process of $2 \mathrm{Mn}^{3.5+} \rightarrow \mathrm{Mn}^{3+}+\mathrm{Mn}^{4+}$ in $\mathrm{La}_{0.5} \mathrm{Ca}_{0.5} \mathrm{MnO}_{3}$, requires a large amount of energy which is about $10 \mathrm{eV}$ (the difference between the $3^{\text {rd }}$ and $4^{\text {th }}$ ionisation energies of manganese). But, the largest value of the Jahn-Teller splitting that has been suggested is about $1 \mathrm{eV}$.

Thus, charge ordering could be described more precisely into a language of octahedron. In this case, ordering of two different $\mathrm{Mn}$-sites are described: $\mathrm{Mn}^{3+}$ which has a distorted octahedron of oxygen ions, and $\mathrm{Mn}^{4+}$ which has a more regular octahedron. RodriguezCarvajal et al. [104] have performed a single crystal study of the charge ordered state of $\mathrm{Pr}_{0.6} \mathrm{Ca}_{0.4} \mathrm{MnO}_{3}$. They reached to the conclusions which were completely different to the conventional charge ordering picture. They have shown that all the oxygen octahedra were elongated and had very similar bond lengths rather than having both elongated $\left(\mathrm{Mn}^{3+}\right)$ and regular $\left(\mathrm{Mn}^{4+}\right)$ octahedra. The authors suggested that the charge separation is as little as 0.01 electrons rather than 1 electron.

It is obvious that the ordering state is due to the correlation at the atomic level, between the physical entities such as: Hund's coupling between $e_{g}$ electrons and a classical spin of core-like $t_{2 g}$ electrons, Heisenberg antiferromagnetic coupling between the core-like electrons in the $t_{2 g}$ shell, hopping between the delocalized $e_{g}$ electrons, electron and JahnTeller phonon coupling, Coulomb interaction between occupied and unoccupied $d$-electrons $[2,3,105]$. Therefore, doping strongly affects the magnetic properties of the manganites. In the following, these quantities will be discussed. It is important to mentioned that more than two Mn-sites are necessary to explain the observed structural modulation in the charge ordered state.

\subsection{Models and parameters}

In the previous sections, the experimental and theoretical works on $\operatorname{Pr}_{1-x} \mathrm{Ca}_{x} \mathrm{MnO}_{3}$ has been reviewed. It is of major interest how physical quantities are incorporated in the model Hamiltonian for this compound. To address this, Tight binding model of perovskites have been developed on the basis of the ab-initio calculations presented here. These have then be used to explore the phase diagram of $\mathrm{Pr}_{1-x} \mathrm{Ca}_{x} \mathrm{MnO}_{3}$ in detail [106].

\subsection{Electronic structure of $\operatorname{Pr}_{1-x} \mathrm{Ca}_{x} \mathrm{MnO}_{3}$}

In this section, we present a theoretical investigation on the structural, electronic, and magnetic properties of the manganite series $\operatorname{Pr}_{1-x} \mathrm{Ca}_{x} \mathrm{MnO}_{3}$, using local hybrid densityfunctional calculations. This functional contains a free parameter per atom type which 
is determined by experimental x-ray photoelectron spectroscopy (XPS). As a first step to study the effects of doping, the series $\operatorname{Pr}_{1-x} \mathrm{Ca}_{x} \mathrm{MnO}_{3}$ for $x=0, \frac{1}{2}, 1$ have been studied.

Before going into the details, the gross features of the atomic and the electronic structure of the $\operatorname{Pr}_{1-x} \mathrm{Ca}_{x} \mathrm{MnO}_{3}$ will be discussed.

\subsubsection{Stoichiometric $\mathrm{CaMnO}_{3}$}

$\mathrm{CaMnO}_{3}$ is the most simple member of the $\operatorname{Pr}_{1-x} \mathrm{Ca}_{x} \mathrm{MnO}_{3}$ manganite. This compound has a orthorhombic perovskite structure. $\mathrm{Mn}$ and $\mathrm{O}$ ions form a network of corner-sharing $\mathrm{MnO}_{6}$ octahedra with a formal valence of $4+$ for the $\mathrm{Mn}$ cations. Thus, the $\mathrm{Mn}$ atoms have a $3 d^{3}$ configuration. It means 3 electrons in the $t_{2 g}$ orbitals and a strong crystal field splitting with the empty $e_{g}$ states. Ca donates its two valence electrons to the valence band of O- $2 p$ character. In the $\mathrm{CaMnO}_{3}$, the size of the Ca ions is sufficiently small so that the $\mathrm{MnO}_{6}$ octahedra tilt to increase the ionic attraction. The orientation of the tilt axis is determined by a bond angle force at the oxygen bridge.

The spins of the three filled $t_{2 g}$ orbitals are parallel. The Mn ions have a formal spin of $\frac{3}{2} \hbar$, and the spins are antiferromagnetically aligned with nearest neighbors. Following the notation of Wollan and Koehler [81], the ground state of $\mathrm{CaMnO}_{3}$ is found to be a G-type antiferromagnet. The Mn-O bond lengths are similar in this structure so, $\mathrm{O}$ cages are not JT distorted.

The calculated DoS for the G-type spin configuration of $\mathrm{CaMnO}_{3}$ is shown in Fig. 3.9. The valence band is mainly attributed to the $\mathrm{O}-2 p$ character with some contribution of Mn-3d orbitals. The valence band extends from $-5 \mathrm{eV}$ to $0 \mathrm{eV}$. The Ca- $d$ states are located above the valence band which is extended from $7 \mathrm{eV}$ to $9 \mathrm{eV}$. Between these two features, the O- $2 p$ valence band and the empty Ca- $d$ states, the Mn- $3 d$ states dominate the conduction band. These states are relevant for the complex properties of manganites.

The Mn-3d orbitals are divided into the three non-bonding $t_{2 g}$ orbitals and the two antibonding $e_{g}$ orbitals, which is originated from the octahedral crystal-field splitting.

In order to understand the bonding and antibonding character of the states, CrystalOrbital Overlap populations (COOP) are used to analyse the band structure energy. This can be distinguished well in the off-diagonal elements of the density of states of $\mathrm{CaMnO}_{3}$ shown in Fig. 3.9. Bonding states are represented with positive sign to the COOP, while antibonding states are contributed with negative sign. In the bottom part of the $\mathrm{O}-2 p$ valence band, the bonding states between Mn-3d and O- $2 p$ are located. It extends between $-5 \mathrm{eV}$ and $-3 \mathrm{eV}$. The relative antibonding states, between $\mathrm{Mn}-3 d$ and $\mathrm{O}-2 p$, extend from $-3 \mathrm{eV}$ to about $6 \mathrm{eV}$. The minority-spin bonding $t_{2 g}$ states are split off from the $e_{g}$ states and lie in the energy region from $-3 \mathrm{eV}$ to $-1 \mathrm{eV}$.

It should be noted that for transition-metal compounds, the bonding states are attributed to the anion, the O-atoms, and the antibonding Mn-O states are attributed entirely to the Mn-ion. This naming convention related to the oxidation number [107] shall not be confused with the true physical nature of the states. 


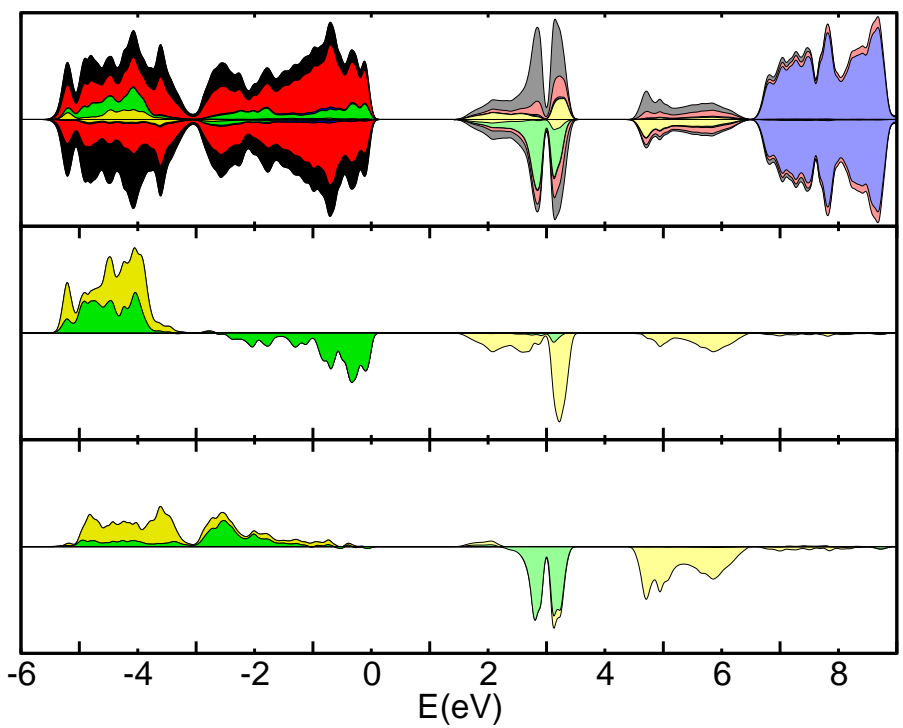

Fig. 3.9: Total and projected $\mathrm{DoS}$ of $\mathrm{CaMnO}_{3}$. The top graph shows the total DoS (black) and the projected DoS for O-p (red), Mn- $t_{2 g}$ (green), Mn- $e_{g}$ (yellow), Ca- $d$ (blue). Projected DoS are stacked ontop of each other. Opposite sign shows the two spin densities. For the Mn-ions only one majority spin direction is considered to the projected DoS. The middle graph shows the COOP between a $\mathrm{Mn}-t_{2 g}$ orbital and an $\pi$-bonded O- $p$ state (green) and the COOP between a Mn- $e_{g}$ orbital and an $\sigma$-bonded $p$-state (yellow) for the majorityspin direction. The two COOPs are stacked ontop of each other. The bottom graph shows the same information in the middle graph but for the minority-spin direction. The valenceband top is aligned to the zero energy. Empty states are filled with the corresponding lighter color than filled states [63].

The antibonding states of the $e_{g}$ orbitals lie energetically above the $t_{2 g}$ electrons. This is originated from the crystal field splitting. This energy separation of $t_{2 g}$ and $e_{g}$ orbitals is due to the covalent interaction with the oxygen neighbors [91]. The three filled $M n-t_{2 g}$ states, which are Mn-O antibonds, lie within the $\mathrm{O}-2 p$ valence band from $-3 \mathrm{eV}$ to $0 \mathrm{eV}$. The majority $\mathrm{Mn}-e_{g}$ states are separated by $1.5 \mathrm{eV}$ from the $\mathrm{O}-2 p$ valence band. These states form the lowest unoccupied conduction band edge of $\mathrm{CaMnO}_{3}$. The minority $\mathrm{Mn}-t_{2 g}$ states are located at $3 \mathrm{eV}$ above the $\mathrm{O}-2 p$ valence band. The minority $\mathrm{Mn}-e_{g}$ states lie from $4 \mathrm{eV}$ to $6 \mathrm{eV}$. The $d$-electrons in the majority-spin and minority-spin direction are spin-splitting due to the Hund's exchange coupling.

\section{Ordering of Mn spin}

The low-temperature orthorhombic phase of $\mathrm{CaMnO}_{3}$ has a G-type ordering of the spins. However, to get more insight, other magnetic orders such as C-, A- and B-type spin configurations are also considered as shown in Fig. 3.10. The spin configurations adopted 
have a significant effect on the density of states. These effects of magnetic ordering can be summarized as:

- Magnetic ordering affects Mn- $d$ states.

- Band gap broadens with decreasing ferromagnetic environment.

- Broadening of $e_{g}$ and $t_{2 g}$ states by increasing ferromagnetic order.

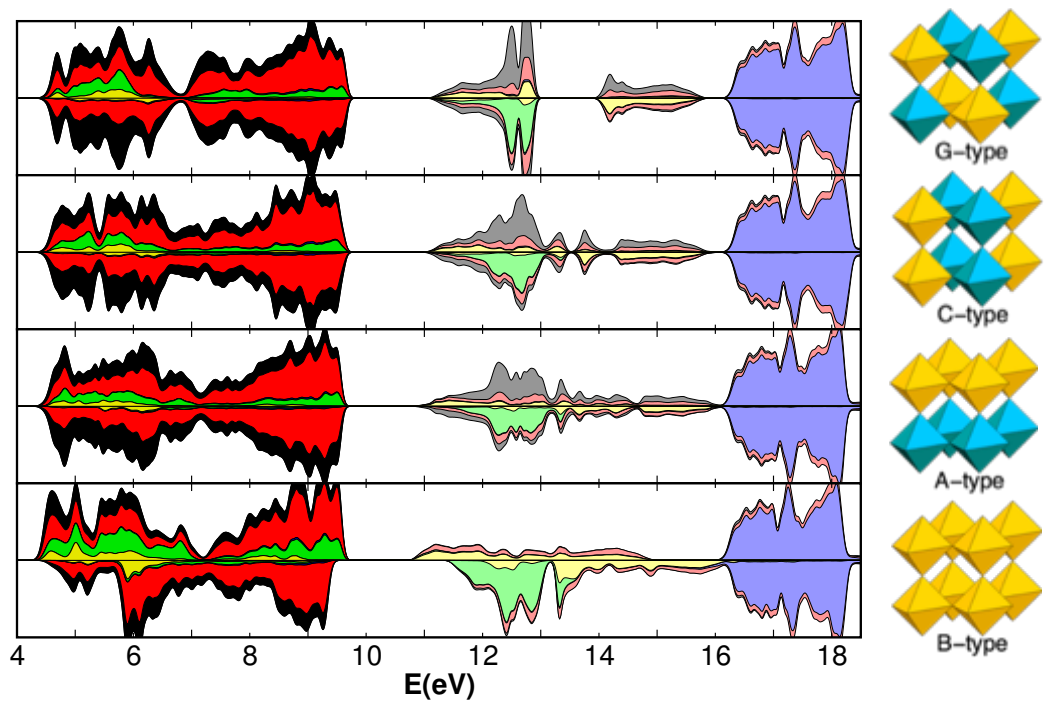

Fig. 3.10: Total and projected density of states for $\mathrm{CaMnO}_{3} \mathrm{G}-, \mathrm{C}-$-, A-, and B-type from top to bottom respectively. The total density of state is black. The area filled in the red is the oxygen contribution, green is the $\mathrm{Mn}-t_{2 g}$, yellow area is the $\mathrm{Mn}-e_{g}$ and blue area is $\mathrm{Ca}-d$ contribution. The empty states are filled with the corresponding lighter color.

The Ca- $d$ states have a same contribution in all magnetic ordering of spins. This shows that the effects of magnetic ordering on the $\mathrm{Ca}$ atoms are nothing. The $\mathrm{O}-2 p$ valence band states have also no influences from different ordering of spins. But in between these two features, Mn-3d states are relevant for the complex properties of $\operatorname{Pr}_{1-x} \mathrm{Ca}_{x} \mathrm{MnO}_{3}$ manganite. Electronically, doping and external magnetic field affect the Mn-3d states which create vast variety of different phases.

The hopping amplitude for carriers to move from Mn to Mn naturally increases as the ferromagnetic order increases. In other words, the hopping amplitude becomes more when the Mn atoms have a same ordering of spins. The Mn-3d states get broadening due to the more carriers hopping. This affects also the band gap of the system. For this reason, the B-type FM configuration has smaller band gap comparison to AF spin configurations.

\section{Structural properties}

For $\mathrm{CaMnO}_{3}$ compounds the Pbnm orthorhombic crystallographic cells are used. The experimental lattice constants are taken from Poeppelmeier et al. [108]. At temperature 
below $T_{N}=120 \mathrm{~K}$, orthorhombic phase of $\mathrm{CaMnO}_{3}$ has a G-type ordering of the spins with the $1.55 \mathrm{eV}$ experimental optical gap [109]. Here, the G-, C-, A-, and B-type spin configurations are considered. The energy difference per formula unit $\Delta E(\mathrm{meV})$ with respect to the ground state is reported in Tab. 3.2. The G-, and C-type AF ordering are the most energetically preferable, the B-type FM configuration has the highest total energy . As seen in Tab. 3.2, the spin configurations do not have a significant effect on the structural parameters of $\mathrm{CaMnO}_{3}$.

Table 3.2: Mean Mn-O bond-length $d_{M n-O}$, Mn-O-Mn bond angle for oxygen bridges in the $a b$-plane $\phi_{O(2)}$ and along the $c$-axis $\phi_{O(1)}$, magnetic moment $\mu$ and number of electrons in the Mn- $d$-shell. The E-type magnetic order of $\operatorname{Pr}_{1 / 2} \mathrm{Ca}_{1 / 2} \mathrm{MnO}_{3}$ uses the experimental lattice constants for $x=0.4$ [40]. Calculated relative energies and fundamental band gaps in $\mathrm{Pr}_{1-x} \mathrm{Ca}_{x} \mathrm{MnO}_{3}$ for different magnetic orders and doping. For each doping the most stable configuration has been chosen as the energy zero.

\begin{tabular}{|c|c|c|c|c|c|c|c|c|c|}
\hline Compound & $\begin{array}{l}\text { Magne } \\
\text { order }\end{array}$ & ${ }_{d_{M n, O}}[\AA]$ & $Q[\AA]$ & $\phi_{O(1)}$ & $\phi_{O(2)}$ & $\mu\left[\mu_{B}\right]$ & $N_{d}$ & $\begin{array}{l}\text { Energy } / A B O \\
(\mathrm{meV})\end{array}$ & $\begin{array}{l}\begin{array}{l}\text { Band } \\
{ }^{3} \text { gap } \\
(\mathrm{eV})\end{array} \\
\end{array}$ \\
\hline \multirow[t]{4}{*}{$\mathrm{PrMnO}_{3}$} & G & 2.044 & 0.470 & $148.3^{\circ}$ & $146.3^{\circ}$ & 3.662 & 3.662 & 22 & 1.31 \\
\hline & $\mathrm{C}$ & 2.045 & 0.460 & $147.7^{\circ}$ & $146.5^{\circ}$ & 3.702 & 4.903 & 29 & 0.90 \\
\hline & $\mathrm{A}$ & 2.039 & 0.406 & $148.2^{\circ}$ & $147.8^{\circ}$ & 3.772 & 4.903 & 0 & 1.05 \\
\hline & B & 2.039 & 0.392 & $147.7^{\circ}$ & $148.0^{\circ}$ & 3.819 & 4.902 & 10 & 1.33 \\
\hline \multirow[t]{6}{*}{$\mathrm{Pr}_{1 / 2} \mathrm{Ca}_{1 / 2} \mathrm{MnO}_{3}$} & G & $2.003 / 1.933$ & $0.056 / 0.100$ & $148.6-156.0^{\circ}$ & $147.5-153.2^{\circ}$ & $3.686 / 2.717$ & $4.887 / 4.811$ & 104 & 0 \\
\hline & $\mathrm{C}$ & $1.940 / 1.997$ & $0.098 / 0.122$ & $150.5-154.1^{\circ}$ & $148.9-151.5^{\circ}$ & $2.885 / 3.636$ & $4.818 / 4.884$ & 96 & 0 \\
\hline & $\mathrm{A}$ & $1.954 / 1.979$ & $0.056 / 0.100$ & $151.5-154.4^{\circ}$ & $149.8-152.5^{\circ}$ & $3.299 / 3.487$ & $4.835 / 4.869$ & 54 & 0 \\
\hline & B & $1.966 / 1.966$ & $0.020 / 0.016$ & $150.6-153.7^{\circ}$ & $150.5-153.0^{\circ}$ & $3.474 / 3.481$ & $4.856 / 4.857$ & 29 & 0 \\
\hline & $\mathrm{CE}$ & $1.991 / 1.943$ & $0.259 / 0.053$ & $156.0-147.8^{\circ}$ & $146.6-157.1^{\circ}$ & $3.526 / 3.125$ & $4.880 / 4.823$ & 0 & 0.57 \\
\hline & $\mathrm{E}$ & $2.014 / 1.951$ & $0.244 / 0.044$ & $149.5-153.8^{\circ}$ & $146.5-154.9^{\circ}$ & $3.634 / 2.975$ & $4.880 / 4.813$ & - & - \\
\hline \multirow[t]{4}{*}{$\mathrm{CaMnO}_{3}$} & G & 1.927 & 0.016 & $151.0^{\circ}$ & $150.3^{\circ}$ & 2.837 & 4.791 & 0 & 1.47 \\
\hline & $\mathrm{C}$ & 1.927 & 0.012 & $150.4^{\circ}$ & $150.4^{\circ}$ & 2.853 & 4.793 & -1 & 1.42 \\
\hline & $\mathrm{A}$ & 1.928 & 0.018 & $151.5^{\circ}$ & $149.6^{\circ}$ & 2.884 & 4.793 & 7 & 1.41 \\
\hline & B & 1.929 & 0.014 & $150.4^{\circ}$ & $149.7^{\circ}$ & 2.922 & 4.794 & 20 & 1.33 \\
\hline
\end{tabular}

At higher temperature (more than $T_{N}$ ), this compound behaves as a paramagnetic while it has a same orthorhombic structure. In the $\mathrm{CaMnO}_{3}$ structure, octahedral tilting is a significant ingredient. The structure and electronic properties (various band gap, density of states, and effective carrier mass) are closely associated with the amount of tilting. This allows to increase the atom density and therefore improve the Coulomb attraction energy for the ions. This effect leads to bending of the oxygen bridges, which in turn reduces the hopping matrix elements between the $e_{g}$ orbitals on neighboring Mn-sites. It can strongly influences the electronic bandwidth of the relevant conduction bands. It should be noted that the bond angle distortion decreases the one-electron bandwidth since the interaction between the neighboring Mn-site is govern by super transfer process via O- $2 p$ states.

The Mn magnetic moment, $2.837 \mu_{\beta}$, is a little higher comparison to the experimental value of $2.65 \mu_{\beta}$ [81] and local spin-density approximation (LSDA) [110] results. Certainly 
due to the covalent bonding of $\mathrm{Mn}-\mathrm{O}$, Mn magnetic moments are below of the net $3 \mu_{\beta}$ of $\mathrm{Mn}^{4+}$ ion. Mn magnetic moment depends on the spin configuration. It is relevant to the correlation between chemical bonding and magnetic interaction. The Mn-O chemical bond specifies the coupling between Mn local spins [85].

\subsubsection{Stoichiometric $\mathrm{PrMnO}_{3}$}

The same ionic radius for $\operatorname{Pr}^{3+}(0.99 \AA)$ and $\mathrm{Ca}^{2+}(1.00 \AA)$ [111] leads to a similar crystal structure of the $(\mathrm{Pr}, \mathrm{Ca}) \mathrm{MnO}_{3}$. The ions are in the formal oxidation states $\mathrm{Pr}^{3+} \mathrm{Mn}^{3+} \mathrm{O}_{3}^{2-}$. Each $\mathrm{Mn}$ atom is surrounded by six oxygen ions in the octahedral environment. Instead of the di-valent $\mathrm{Ca}$ ions, the tri-valent Pr ions are replaced. The additional electrons are inserted into the empty majority $\mathrm{Mn}-e_{g}$ states. The doubly degenerate $e_{g}$ states is lifted by Jahn-Teller (JT) distortion of the $\mathrm{MnO}_{6}$ octahedra. Due to the JT distortion, there are two different kinds of $\mathrm{Mn}-\mathrm{O}$ bond lengths in the octahedron cage. They are two long (apical) and two pairs of shorter (equatorial) Mn-O bond lengths. The extra electrons cause the JT distortions and form polarons. The interplay of magnetic and polaron order bring the complexity into the manganites phase diagram.

The calculated DoS for the A-type spin configuration of $\mathrm{PrMnO}_{3}$ is shown in Fig. 3.11. The filled $\operatorname{Pr}-f$ states are located in the upper part of the O- $p$ valence band. It extends from $-2 \mathrm{eV}$ to $-1 \mathrm{eV}$. The empty Pr- $f$ states extend from $2 \mathrm{eV}$ to $4 \mathrm{eV}$ and energetically overlap with the empty Mn-3d states in the conduction band. In contrast to $\mathrm{CaMnO}_{3}$, the majority $\mathrm{Mn}-e_{g}$ orbitals is split off by a distortion of $\mathrm{MnO}_{6}$ octahedra. The energy of the occupied state lowers due to the weakening of the antibonds with the neighboring oxygen atoms. This is caused by increasing of the corresponding Mn-O bond distances. This JT effect opens up the band gap in $\mathrm{PrMnO}_{3}$. The ordering of the JT distortions produces an orbital ordered state.

In this compound, Pr- $f$ states overlap with O- $2 p$ states and located in below and above of the Fermi level that is in agreement with photoemission spectroscopic result which is showing these states should be $2 \mathrm{eV}$ below the Fermi level [112]. The Pr- $d$ states are located from $4 \mathrm{eV}$ to $9 \mathrm{eV}$ above the Pr- $f$ states.

All two compounds, $\mathrm{PrMnO}_{3}$ and $\mathrm{CaMnO}_{3}$, are insulating at low temperature. Additional electrons tend to form polarons. Electrons are stabilized by the lattice distortion and shift the electron level from the conduction band into the valence band. Consequently, a finite band gap remains in the electronic structure. This is in contradiction with the rigidband picture in semiconductor physics where the charge carriers coupling to the lattice distortion is small. Because of the strong correlations, the rigid-band picture breaks down and the environment of the charge carriers responds strongly. So, the additional electrons do not lead to mobile charge carriers. As shown in Fig. 3.11, there is a filled band above the $\mathrm{O}-2 p$ band which is separated from the conduction band. The weight of the filled band increases with the number of electrons on the Mn-site. 


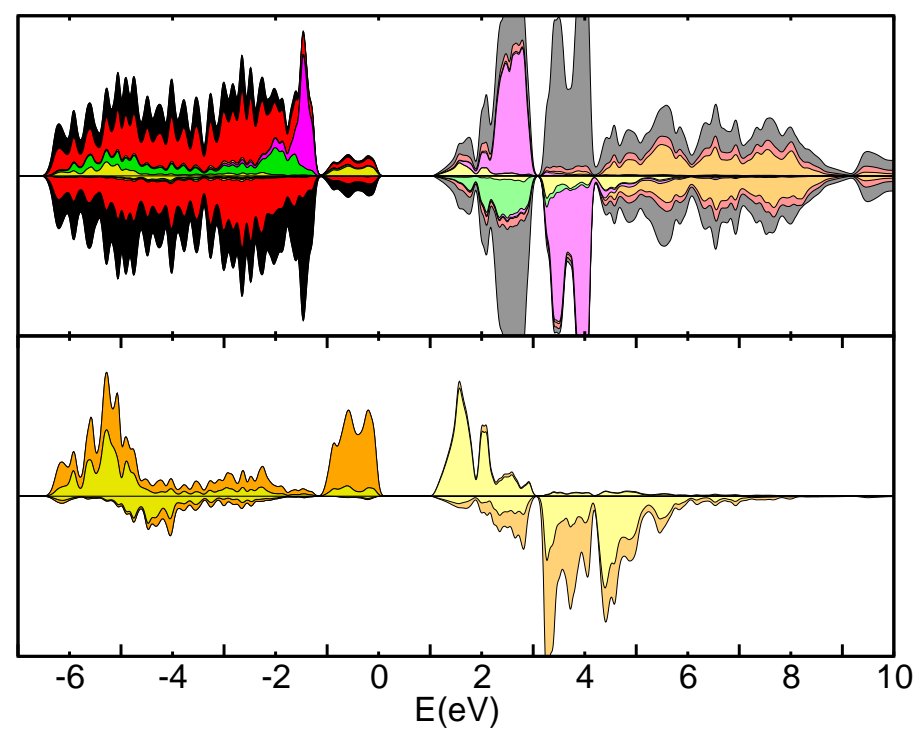

Fig. 3.11: Total and projected $\mathrm{DoS}$ of $\mathrm{PrMnO}_{3}$. Colors are represented based on Fig. 3.9 and also described in the text. The Pr- $f$ states are shown in magenta and the Pr- $d$ states in orange. The bottom graph shows the DoS for the lower (orange) and upper (yellow) $e_{g}$ orbitals, which are split by the Jahn-Teller effect [63].

\section{Ordering of Mn spin}

At low-temperature orthorhombic phase of $\mathrm{PrMnO}_{3}$ has an A-type ordering of the spins. However, to get more insight, other magnetic orders such as G-, C-, and B-type spin configurations are also considered as shown in Fig. 3.12. Analogously, the density of states in $\mathrm{PrMnO}_{3}$ compounds behave similar to $\mathrm{CaMnO}_{3}$ by changing the magnetic orders. As shown in Fig. 3.12, the magnetic order influences the Mn- $d$ states and increasing the ferromagnetic order broadens the $\mathrm{Mn}-e_{g}$ and $\mathrm{Mn}-t_{2 g}$ contributions. Band gap also gets smaller by increasing the ferromagnetic environment.

The Pr- $f$ states have a same contribution in all magnetic ordering of spins. This shows that the magnetic order influences the Mn- $d$ states and it has no effects on the Pr atoms. The O- $2 p$ valence band states have also no influences from different ordering of spins. In other words, Mn-3d states are relevant for the complex properties of $\operatorname{Pr}_{1-x} \mathrm{Ca}_{x} \mathrm{MnO}_{3}$ manganite. Electronically, doping and external magnetic field affect the Mn-3d states which create vast variety of different phases.

The Mn-3d states get broadening due to the more carriers hopping. The hopping amplitude becomes more when the Mn atoms have a same ordering of spins. This affects also the band gap of the system. For this reason, the B-type FM configuration has smaller band gap comparison to AF spin configurations. 


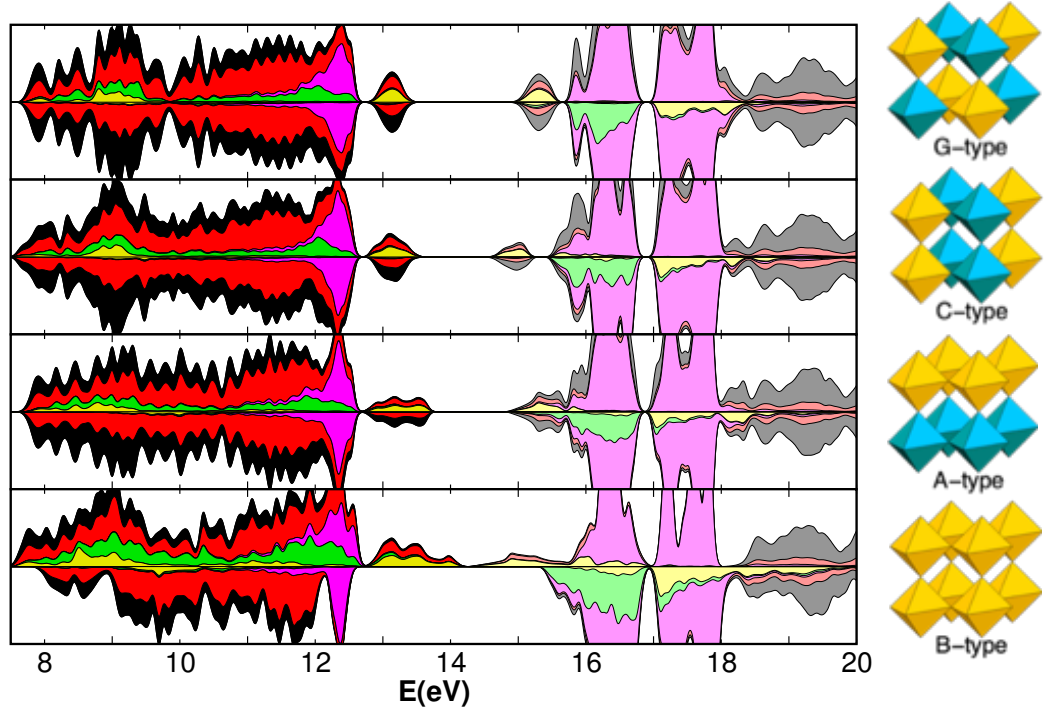

Fig. 3.12: Total and projected density of states for $\mathrm{PrMnO}_{3} \mathrm{G}_{-}, \mathrm{C}-, \mathrm{A}-$, and B-type from top to bottom respectively. The total density of state is black. The area filled in the red is the oxygen contribution, green is the $\mathrm{Mn}-t_{2 g}$, yellow area is the Mn- $e_{g}$ and magenta area is $\operatorname{Pr}-f$ contribution. The empty states are filled with the corresponding lighter color.

\section{Structural properties}

For $\mathrm{PrMnO}_{3}$ compounds the Pbnm orthorhombic crystallographic cells are used. The experimental lattice constants are taken from Alonso et al. [113]. The low-temperature orthorhombic phase of $\mathrm{PrMnO}_{3}$ has an A-type ordering of the spins. Here, the G-, C-, and B-type spin configurations are also considered. The energy difference per formula unit $\Delta E(\mathrm{meV})$ with respect to the ground state is reported in Tab. 3.2. The ground state of $\mathrm{PrMnO}_{3}$ is calculated to be an A-type antiferromagnet with estimated band gap about $1.05 \mathrm{eV}$. The structural parameters are summarized in Tab. 3.2.

The longer Mn-O bond-length in $\mathrm{PrMnO}_{3}$ is primarily attributed to the cooperative JT distortion, which is not present in $\mathrm{CaMnO}_{3}$. The crystal structure of $\mathrm{PrMnO}_{3}$ can be described as a perovskite with tilting that Mn-O-Mn angles become less than $180^{\circ}$. The JT distortion of the octahedra induces one long Mn-O bond and two short bonds. This compound practically shows similar properties as $\mathrm{LaMnO}_{3}$. The only difference is due to the smaller ionic radius of Pr ion compared with La, which displays more amount of the tilting of the oxygen octahedra in orthorhombic Pbnm crystal structure. This effect induces the smaller effective $e_{g}-e_{g}$ hopping between Mn atoms and narrower band width.

The tilt angle in $\mathrm{PrMnO}_{3}$ is higher than $\mathrm{CaMnO}_{3}$ as shown in Tab. 3.2. This difference is due to the introducing of electrons, and consequently JT distortion, and changing over the Mn-O bonds.

The Mn magnetic moment of $\mathrm{PrMnO}_{3}$ is about 3.77 $\mu_{\beta}$. Jirak et al. [4] reported a value of $3.54 \mu_{\beta}$. Due to the covalent bonding Mn-O, Mn magnetic moments are below of the net 
of $\mathrm{Mn}^{3+}$ ion. It should be noted that Mn magnetic moment depends on spin configuration, and it is relevant to the correlation between chemical bonding and magnetic interaction.

\subsubsection{Half-doped $\mathrm{Pr}_{1-x} \mathrm{Ca}_{x} \mathrm{MnO}_{3}$}

\section{Zener polaron}

In $\operatorname{Pr}_{1 / 2} \mathrm{Ca}_{1 / 2} \mathrm{MnO}_{3}$, like $\mathrm{PrMnO}_{3}$, each $\mathrm{Mn}$ ion is sixfold coordinated with $\mathrm{O}$ atoms which form an octahedron cage around the Mn ion. However, In the half-doped system, $\mathrm{Pr}_{1 / 2} \mathrm{Ca}_{1 / 2} \mathrm{MnO}_{3}$, the Jahn-Teller polaron is replaced by a Zener polaron, which is characterized by an electron sharing between the two ferromagnetically coupled Mn neighbors.

In the Zener polaron, two neighboring Mn sites have a same Jahn-Teller expansion along the axis of the pair. This is in contradiction to the orbital ordering in $\mathrm{PrMnO}_{3}$, which exhibits an alternating series of prolate and oblate octahedra in the $a b$-plane direction. This is shown in Fig. 3.13.

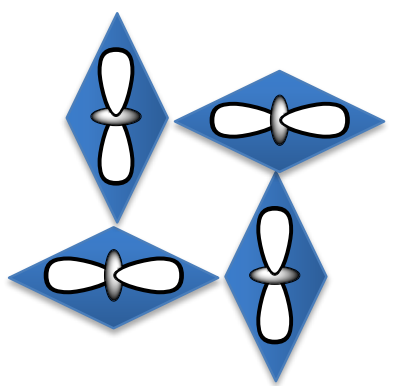

(a)

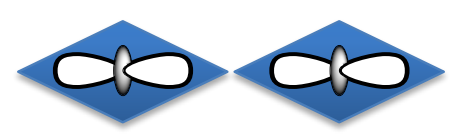

(b)

Fig. 3.13: Schematic drawing of the (a) orbital ordering in $\mathrm{PrMnO}_{3}$ and (b) Zener-polaron in $\operatorname{Pr}_{1 / 2} \mathrm{Ca}_{1 / 2} \mathrm{MnO}_{3}$. Shown is the $a b$-plane.

The band gap in $\mathrm{PrMnO}_{3}$ is due to Jahn-Teller splitting while in $\mathrm{Pr}_{1 / 2} \mathrm{Ca}_{1 / 2} \mathrm{MnO}_{3}$ the band gap occur between filled and empty states of lower Jahn-Teller band. In the filled lower band states, the two Mn- $e_{g}$ orbitals on the dimer point towards the central oxygen. These two $e_{g}$ orbitals have even parity which prohibits any interaction with the central oxygen ion. The corresponding antibonding state forms the unoccupied part and locates above the filled band. The splitting of the lower Jahn-Teller band is originated from the antibond with the bridging oxygen ion. This is shown in Fig. 3.14. Irrespective of the type of Jahn-Teller distortions, the lowest wave function is a bonding sigma orbital, which is non-bonding with respect to the O- $p$ orbital. If both octahedra are prolate along the dimer axis as shown in Fig. 3.14, the sigma orbitals are already lower resulting in a lower lying, and thus more stable wave function for the electron. It is this effect that favors this particular cooperative Jahn-Teller distortion, which otherwise would be unfavorable due to the strain imposed. 


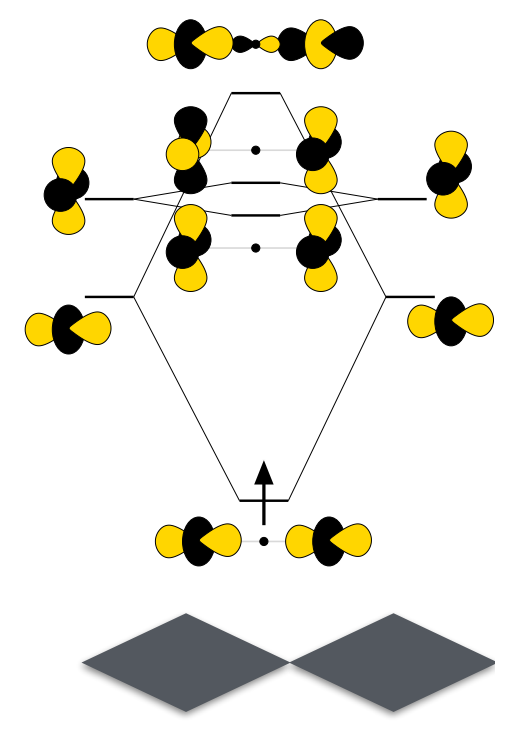

Fig. 3.14: Orbital diagram of a Zener polaron. The orbital diagram is for two prolate octahedron along the dimer axis [87].

Only the antibonding sigma orbital has a symmetry to accommodate an oxygen orbital. This wave function is therefore denoted as oxygen hole state. As the total weight of an orbital, filled and empty, equal unity, the contribution of the oxygen orbital to an empty wave function implies that this amount is missing in the occupied wave functions. This deficiency of the O- $p$ electrons is described as an oxygen hole.

\section{Ordering of Mn spin}

The experimental lattice constants are taken from Jirak et al. [4] for $\operatorname{Pr}_{1 / 2} \mathrm{Ca}_{1 / 2} \mathrm{MnO}_{3}$ for G-type, C-type, A-type and the ferromagnetic B-type order. Two further calculations have been performed for the half-doped material. For $x=0.4$, the experimental lattice constants [40] are used which has a $P 21 \mathrm{~nm}$ space group. For $\operatorname{Pr}_{1 / 2} \mathrm{Ca}_{1 / 2} \mathrm{MnO}_{3}$ an alternating (rock-salt) ordering of A-type ions $\mathrm{Ca}$ and $\mathrm{Pr}$ are used. For the CE-type order also one calculation with layers of $\mathrm{Pr}$ and $\mathrm{Ca}$ ions are performed with alternating in $c$-direction.

Calculations of the E-type and the CE-type ordering are performed with the measured lattice constants [40]. The experiments have been performed for $x=0.4$ which is away from half doping. This explains that the lattice constants are larger than the exact half doping. We use the experimental lattice constants albeit with electron occupations according to precise half-doping.

Our calculations yield the CE-type order more stable than the E-type order by $30 \mathrm{meV}$ per Mn-site. While not being the most stable order, its understanding is nevertheless 
important for several reasons: The energy difference is in the range of thermal energies so that this structure is likely to contribute to the randomization of the spin order at higher temperatures. It is also conceivable that doping or external fields interchange the stability.

While we find surprisingly similar (within $15 \%$ ) Jahn-Teller distortions in the two magnetic orders, the E-type order exhibits a strong charge order as seen in the magnetic moments listed in Tab. 3.2.

The prototypical order of Zener polarons at half filling is related to the E-type magnetic structure shown in Fig. 3.15. We find this magnetic order higher in energy compared to the CE-type magnetic structure. In the CE-type magnetic structure the Zener-polarons polymerize, the material exhibits one-dimensional zig-zag chains of ferromagnetically coupled Mn-ions.

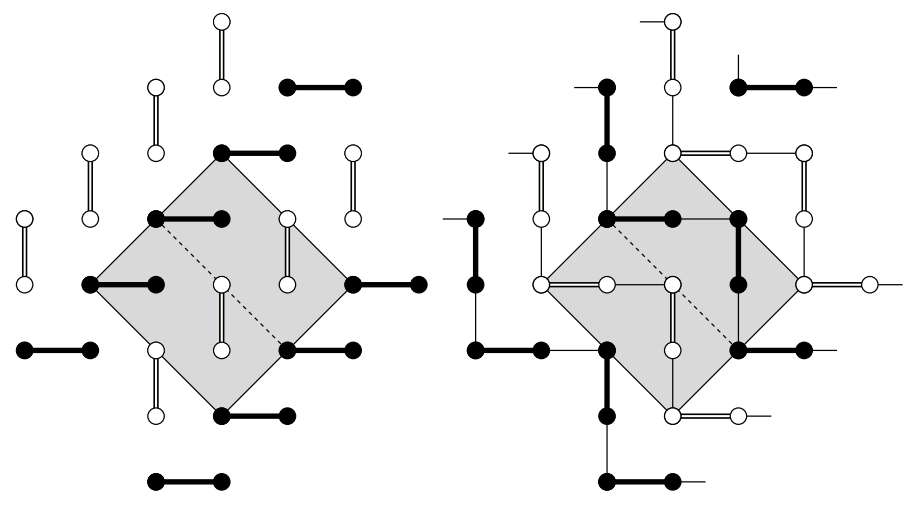

Fig. 3.15: Arrangements of the Zener-polaron for $\operatorname{Pr}_{1 / 2} \mathrm{Ca}_{1 / 2} \mathrm{MnO}_{3}$ in the E-type (left) and the CE-type (right) magnetic order. Black and white spheres indicate opposite spin directions. Shown is the $a b$-plane. Different planes are coupled to each other antiferromagnetically [63].

The calculated DoS for the CE-type spin configuration of $\operatorname{Pr}_{1 / 2} \mathrm{Ca}_{1 / 2} \mathrm{MnO}_{3}$ is shown in Fig. 3.16. A fundamental band gap of $0.57 \mathrm{eV}$ and a direct band gap of $0.72 \mathrm{eV}$ are obtained for the $\operatorname{Pr}_{1 / 2} \mathrm{Ca}_{1 / 2} \mathrm{MnO}_{3}$. The G-, C-, and A-type AF and the FM B-type order are also considered. The energy difference per formula unit $\Delta E(\mathrm{meV})$ with respect to the ground state is listed in Tab. 3.2. All simple magnetic orders (G-, C-, A- and B-type) are energetically unfavorable and do not have a finite band gap in our calculations. The calculated band gap of $0.57 \mathrm{eV}$ for the CE-type $\mathrm{Pr}_{1 / 2} \mathrm{Ca}_{1 / 2} \mathrm{MnO}_{3}$ is larger than the reported experimental value of $0.37 \mathrm{eV}$ [46] at $80 \mathrm{~K}$ for doping $x=0.5$. This value has been obtained from the extrapolated onset of the photoconductivity. The CO gap at $x=0.5$ is estimated to be $0.15 \mathrm{eV}$ at $80 \mathrm{~K}$ [114], and the optical gap is found to be $0.18 \mathrm{eV}$ at $10 \mathrm{~K}$ for $x=0.4$ [115].

Our calculations of $\mathrm{Pr}_{1 / 2} \mathrm{Ca}_{1 / 2} \mathrm{MnO}_{3}$ in the CE-type magnetic structure produce only a small asymmetry in the segment of its zig-zag chains. This asymmetry is a precondition for the observed finite electric polarization. The experimental structure by Daoud-Aladine et al., however, [40] reveals a clear asymmetry. Previous calculations [43] did not optimize the 


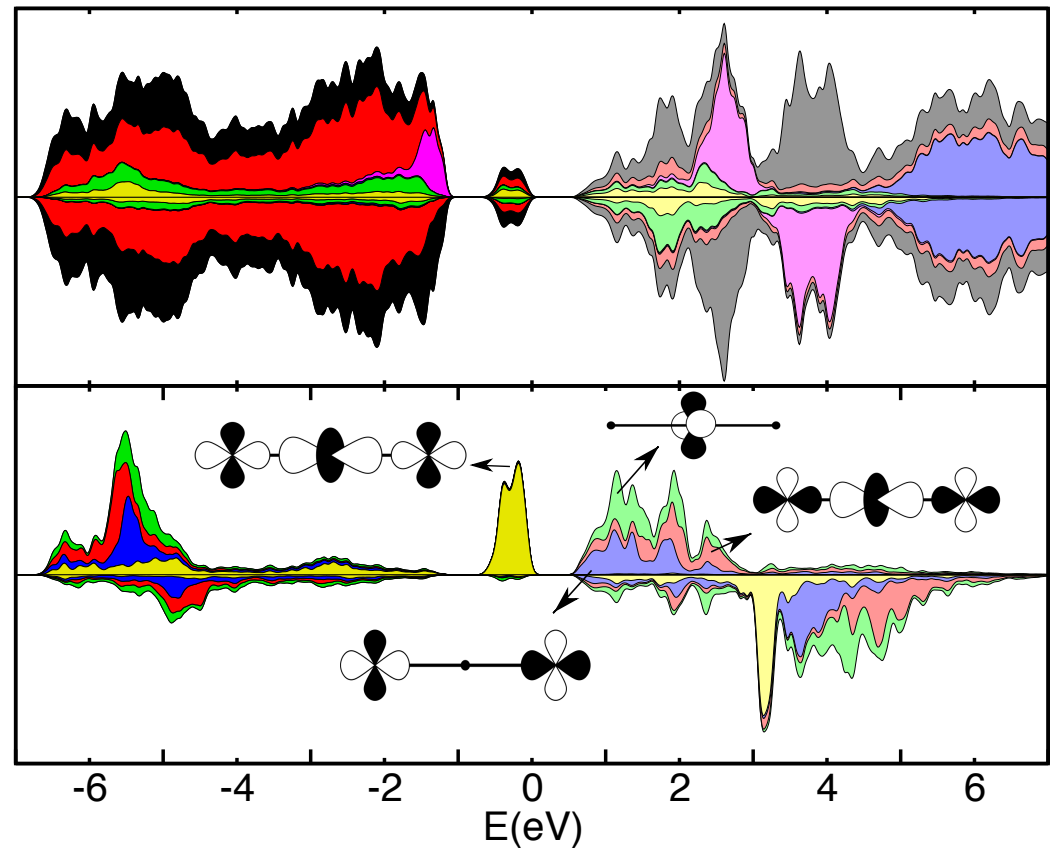

Fig. 3.16: Total and projected $\mathrm{DoS}$ of $\operatorname{Pr}_{1 / 2} \mathrm{Ca}_{1 / 2} \mathrm{MnO}_{3}$ in the CE-type antiferromagnetic order. In the top graph colors are represented based on Fig. 3.9 and Fig. 3.11 and also described in the text. The bottom graph shows the projection on Wannier-like orbitals. The orbital $\left|w_{1}\right\rangle$ forming the occupied $e_{g}$ band is shown in yellow. The optically active excited orbital $\left|w_{2}\right\rangle$ is shown in blue. The fully antibonding orbital $\left|w_{3}\right\rangle$, shown in red, is optically inactive. The green area refers to the orbital $\left|w_{4}\right\rangle$ with $\delta$-symmetry on the central atom [63].

atomic structure and thus recovered the asymmetry from the experiment. An asymmetry can, however, be induced by a magnetic structure different from the CE-type order, which naturally groups together pairs of Mn-ions to Zener polarons. One candidate is the E-type order sketched in Fig. 3.15.

The transition from the CE-type magnetic pattern to the E-type magnetic pattern differs from the one investigated by Giovanetti [44]. Both transitions are the result of a spin rotation of Mn-dimers. The spin rotation investigated by Giovanetti leads back to a displaced CE-type structure for a rotation angle of $180^{\circ}$, while the E-type structure is a distinct magnetic ordering.

The symmetry adapted orbitals, that allow one to rationalize the electronic structure, are listed below. 


$$
\begin{aligned}
& \left|w_{1}\right\rangle=\left|l, d_{3 x^{2}-r^{2}}\right\rangle \cos (\beta)+\left|r, d_{3 x^{2}-r^{2}}\right\rangle \sin (\beta) \\
& \left|w_{2}\right\rangle=\left|l, d_{3 x^{2}-r^{2}}\right\rangle \sin (\beta)-\left|r, d_{3 x^{2}-r^{2}}\right\rangle \cos (\beta) \\
& \left|w_{3}\right\rangle=\left|l, d_{y^{2}-z^{2}}\right\rangle \\
& \left|w_{4}\right\rangle=\left|r, d_{y^{2}-z^{2}}\right\rangle
\end{aligned}
$$

The determined angle from the calculated DoS is $\beta=25^{\circ}$, which places $80 \%$ of the electron on one site. This reflects the strong charge order in the E-type structure.

The charge order is related to a pattern of Jahn-Teller distortions, where the amplitude is determined by the charge order, while the orientation is determined by the magnetic structure.

The charge order in combination with the E-type magnetic structure results in a strong electric polarization of each Mn-pair forming a Zener polaron. The charge order can develop in two different ways, and we expect the system to be bistable. We have not detected an internal mechanism that favors one or the other orientation of the polarization.

The asymmetry of the dimers due to charge order has not been seen in experiment. It is conceivable that both configurations coexist at the temperature of the experiment, so that an average is observed.

While the E-type structure is found to be only metastable, it may be thermally accessible and, together with the non-collinear orders suggested by Giovanetti [44], may contribute to the order parameter for the phase transition into the paramagnetic state with shortrange antiferromagnetic order. All the structures would leave the ferromagnetic alignment within the Zener polaron intact and none of the structures results in a closing of the band gap.

The band gap of the E-type order is with $0.63 \mathrm{eV}$ only $0.04 \mathrm{eV}$ smaller than that of the CE-type order as calculated with the same lattice constants. The conduction band minimum is dominated by state $\left|w_{3}\right\rangle$. The recombination into the ground state is dipole forbidden, lending support to potentially long-lived electronic excitations.

\subsection{Experimental verification of the calculations}

Before discussing the relevance of our findings to experiment, we review the role of the Fock admixture in $\mathrm{CaMnO}_{3}$ and $\mathrm{PrMnO}_{3}$ calculations. This parameter replaces a fraction of the exchange energy $E_{x}^{P B E}$ of the PBE [59] functional by the explicit Hartree-Fock exchange energy $E_{x}^{H F}$. The main effect of admixing the expression in the hybrid functionals is the stabilization of filled orbitals respect to unoccupied orbitals. This is due to the selfinteraction correction. We briefly give an introduction to the hybrid factor weight in theoretical calculations. 


\subsubsection{Role of the $U$-tensor}

Description of materials with strong electron correlations is problematic for conventional functionals. For LDA and GGA many insulating transition metal oxides are predicted as metals and their electronic spectra differ from the excitation spectra. Hybrid functionals turns them into antiferromagnetic insulators, which is a dramatic improvement. For example, in the PBE [59] density functional the band gap of $\mathrm{CaMnO}_{3}$ is obtained due to a transition from the $t_{2 g}$ to $e_{g}$ states. While in reality the band gap of $\mathrm{CaMnO}_{3}$ is a transition from the $\mathrm{O}-2 p$ states to the $e_{g}$ states.

Hybrid functionals contain a fraction of an explicit exchange term which is similar to the screened exchange of the GW method [23]. The GW method has a theoretical basis to predict the spectrum of one-particle excitations. Hybrid functionals produce Kohn-Sham spectra which are closer to excitation spectra.

The main role of the $U$-tensor, the exact exchange contribution, is downward shift of occupied orbitals compared to empty orbitals. Thus, this term opens up a band gap for transition metal oxides. For the manganites this affects mostly the filled Mn- $3 d$ orbitals and the Pr- $f$ orbitals. For this reason, incorporation of a scaled Fock term in the hybrid functional is vital for the present work.

In order to investigate this effect we analyzed the DoS of $\mathrm{CaMnO}_{3}$ and $\mathrm{PrMnO}_{3}$ which is shown in Fig. 3.17, with different values of hybrid mixing factor. It should be noted that the hybrid factors have been kept equal on all atoms.

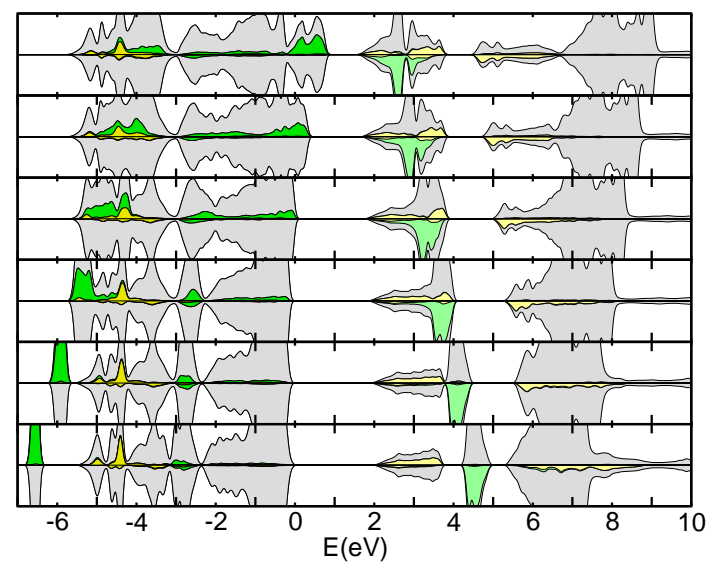

(a)

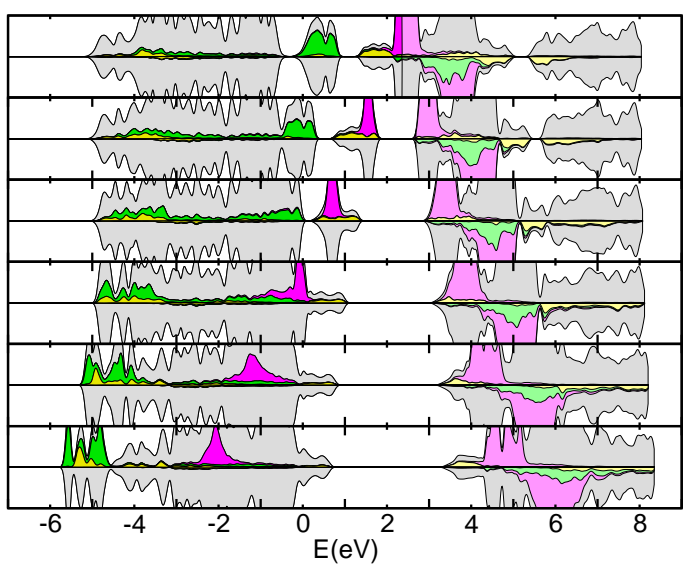

(b)

Fig. 3.17: The total and projected DoS for (a) $\mathrm{CaMnO}_{3}$, and (b) $\mathrm{PrMnO}_{3}$ for hybrid mixing factors from 0 to 0.25 in steps of 0.05 to bottom. The total density of states is grey. The hybrid mixing factors are equal on all atoms. The O- $p$ band top for $a_{x}=0.1$ is aligned to the energy zero [63]. 
For $\mathrm{CaMnO}_{3}$, the hybrid mixing factor shifts the occupied $t_{2 g}$ orbitals downward from above the $\mathrm{O}-2 p$ band to its bottom part. Description of the band gap in a standard DFT functional $\left(a_{x}=0.0\right)$ is incorrect. Without admixture of a Fock term, the band gap is due to a transition from $t_{2 g}$ to $e_{g}$ states. A small contribution of exact exchange, $a_{x}=0.05$, shifts the occupied $t_{2 g}$ orbitals into the oxygen valence band. This changes the band gap description and the material becomes charge-transfer insulator.

In $\mathrm{CaMnO}_{3}$, the $t_{2 g}$ states have two main contributions within the O- $p$ valence band. These two contributions are located in the upper part and the lower part of the oxygen valence band. In addition to the displacement of the $t_{2 g}$ states, a small contribution of exact exchange changes the weight of the two contributions. For a large amount of the Fock term, the $t_{2 g}$ states separate from the bottom part of the oxygen valence band and form a peak. The two $t_{2 g}$ contributions in the valence band separate bonding and antibonding states of $\mathrm{Mn}-t_{2 g}$ with $\mathrm{O}-p$.

Furthermore, the exact exchange admixture in the hybrid functionals changes the Hund's-rule coupling between majority-spin and minority-spin orbitals. The higher values of the Fock term increase the Hund's-rule coupling. This coupling is indicated by keeping the $e_{g}$ orbitals of both spin directions apart. The higher Hund's-rule coupling shows increasing localization of the $t_{2 g}$ orbitals, which increases the local moment.

The Fock admixture has a little influence on the contribution of the O- $p$ valence band. The O- $p$ orbitals are filled and localized, however its position is little affected.

Passing from $\mathrm{CaMnO}_{3}$ to $\mathrm{PrMnO}_{3}$, the Mn- $d$ states have the same behavior on the Fock admixture as shown in Fig. 3.17. An important difference is that the occupied $e_{g}$ orbitals are shifted downward with increasing the Fock admixture as well as the $t_{2 g}$ states.

In $\mathrm{PrMnO}_{3}$, the Pr- $f$ states are present which do not exist in $\mathrm{CaMnO}_{3}$. For a GGA functional, the Fermi level is pinned within the Pr- $f$ states. These states are split into majorityand minority-spin contributions in the GGA calculation, while there is no splitting between the occupied and unoccupied states. A small contribution of the Fock admixture separates the filled and empty Pr- $f$ states. This energy separation of one electron shell is typical for the atoms with strongly interacting electrons. With increasing the Fock admixture, the occupied Pr- $f$ orbitals shift downward and the empty states shift upward by a similar amount.

\subsubsection{XPS}

Now that we have introduced the role of the Fock admixture, the specific admixture for the Fock term will be obtained by comparison our findings with the experimental XPS data. This is presented in Fig. 3.18. The resonant Mn-XPS (green line) shows the $d$ orbitals within the $\mathrm{O}-p$ valence band. It contains a main peaks at $-2 \mathrm{eV}(\mathrm{B})$ and a weaker maximum at $-6 \mathrm{eV}(\mathrm{A})$ on our energy scale. These two features are attributed to the two contributions of the Mn- $d$ orbitals within the oxygen valence band. The presence of a Mn LVV Auger process broadens the weaker maximum (A) which can be separated from the resonant Mn $2 p \rightarrow 3 d$ peak as shown by Waddati et al. [116]. The non-resonant valence 
band photoemission have been discussed for all features by Kurash et al. [117].

An important feature for the manganites is the shoulder (D) which is located between the Fermi level and the upper peak. This feature is attributed to the occupied $e_{g}$ orbitals above the O- $p$ valence band. The weight of the peak grows with increasing number of electrons in the $d$-shell. For this reason, we interpret this feature to the $e_{g}$ orbitals. It should be noted that the experimental data for $\mathrm{CaMnO}_{3}$ are obtained at small Pr concentration $(x=0.80)$. Therefore, the small but non-zero filled $e_{g}$ states are appeared and the feature is not completely vanished.

Presence of the main peak (B) compared to the lower maximum (A), and the existence of the prepeak (D) above the main peak in the Mn-XPS will be obtained with the small Fock admixture on the Mn-site. The value of $a_{x}=0.07$ for the Mn site has been chosen.

The experimental Pr-XPS (magenta) is located between the main peak of the Mn-XPS (B) and the prepeak (D). The filled Pr- $f$ states is observed with a single peak (C) which has a same shape to the Pr-projected DoS. The value of $a_{x}=0.15$ for the Pr site has been chosen.

The commonly used value for the Fock admixture is $a_{x}=0.25$ [61]. It make the Pr- $f$ contributions physically unstable. As shown in Fig. 3.17, it furthermore predicts the spectral properties incorrectly. For this reason, the correct factors are determined by comparison with the experimental XPS data. The comparison with experimental spectra shows that the suggested value of $a_{x}=0.15[31,30]$ is still very high. Recent studies [118, $31,30]$ also have shown the smaller value for the Fock admixture. Our mixing factors are listed in Tab. 3.3.

Table 3.3: Factors $a_{x}$ for the element specific admixture of the Fock term.

\begin{tabular}{|l||c|c|c|c|}
\hline \hline Element & $\mathrm{Pr}$ & $\mathrm{Ca}$ & $\mathrm{Mn}$ & $\mathrm{O}$ \\
\hline$a_{x}$ & 0.15 & 0.1 & 0.07 & 0.1 \\
\hline \hline
\end{tabular}

\subsubsection{ELNES and XANES}

Both O- $K$ ELNES and XANES spectra measure the electric dipole transitions from the $1 s$ core-orbital of oxygen to its unoccupied $p$-states. Therefore, it gives information of the unoccupied density of states which have some O- $p$ character. In $\operatorname{Pr}_{1-x} \mathrm{Ca}_{x} \mathrm{MnO}_{3}$, the antibonding $e_{g}$ states lift some $\mathrm{O}-p$ weight from the valence band into the spectrum of empty states. Thus antibonding states of mainly $\mathrm{Mn}-e_{g}$ character can be probed. The Mn$t_{2 g}$ states have a less contribution to the spectrum, because the $t_{2 g}$ orbitals form weak $\pi$ antibonds. In addition to $\mathrm{Mn}-d$ states, the A-type ions, $\mathrm{Pr}$ and $\mathrm{Ca}$, have some contribution to the spectra. The $d$-electrons of the A-type ions lift some oxygen weight into the empty spectrum with the antibonding character. Thus, another contribution to the spectra is 


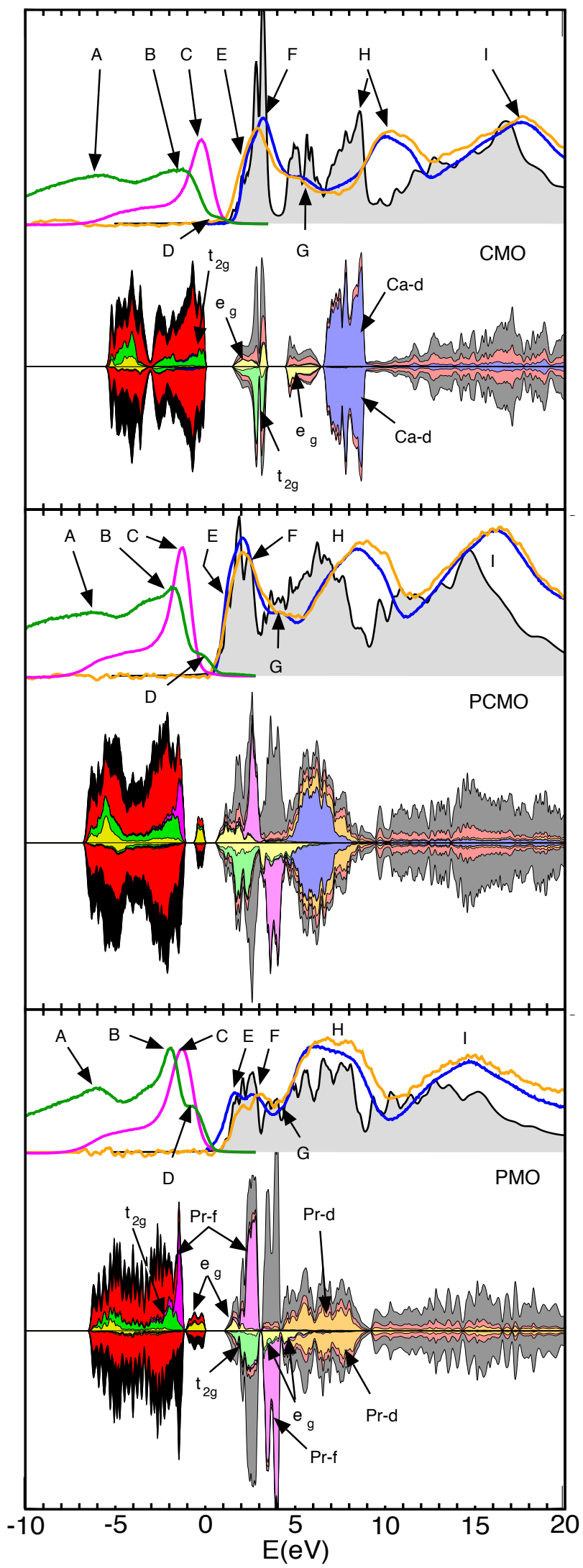

Fig. 3.18: Calculated ELNES spectra (shaded grey) and spin-resolved DoS together with Mn-XPS (green), Pr-XPS (magenta), Xanes (blue), and ELNES (orange). The results are shown for $\mathrm{CaMnO}_{3}$ (top) $(\mathrm{x}=0.8$ for XANES and $\mathrm{x}=0.95$ for ELNES), $\quad \operatorname{Pr}_{1 / 2} \mathrm{Ca}_{1 / 2} \mathrm{MnO}_{3}$ (middle) and $\mathrm{PrMnO}_{3}$ (bottom). Projected DoS are shown for $\mathrm{Mn}-e_{g}$ (yellow), $\mathrm{Mn}-t_{2 g}$ (green), Pr- $f$ (magenta), Ca- $d$ (blue), Pr- $d$ (orange) and O-p (red). The DoS of the two spin directions are indicated with opposite sign. Only the majority-spin direction is shown for Mn and Pr [63]. 
due to the oxygen bridges, which point towards the A-type $d$-states. However, due to the selection rule, the spectra is not able to show the full view of DoS.

The calculated ELNES spectra (shaded gray) compared to the experimental ELNES (orange) and XANES (blue) spectra are shown in Fig. 3.18 alongside with the calculated DoS.

There are three main peaks in the experimental spectra. The lower peak (E, F, and $\mathrm{G}$ ) is located below $6 \mathrm{eV}$ in our energy scale. The middle peak $(\mathrm{H})$ extends from $6 \mathrm{eV}$ to $10 \mathrm{eV}$. The upper peak (I) lies in the energy range from $15 \mathrm{eV}$ to $18 \mathrm{eV}$. We assign the upper peak (I) to a free-electron like feature.

The middle peak $(\mathrm{H})$ is attributed to the Ca- $d$ and Pr- $d$ states. These states overlap with the lone-pairs of the oxygen bridges and contribute to the spectra. The calculated Ca$d$ and Pr- $d$ states are located below the experimental spectra due to a self-energy shift. The self-energy is not described adequately in our DFT calculations. The electron-correlations decrease in the high energy excitation. It means the Coulomb repulsion reduces in the quasi-particle spectrum. But in the DFT calculations the correlations are fully developed. For this reason the calculated states lie lower than the measured excitation levels.

The lower peak (E, F, and $\mathrm{G}$ ) relevant for the polaron physics of manganites lies in the energy range from $1 \mathrm{eV}$ to $6 \mathrm{eV}$. The shoulder between $4 \mathrm{eV}$ and $5 \mathrm{eV}(\mathrm{G})$, which is present for all doping levels, is attributed to the minority-spin $\mathrm{Mn}-e_{g}$ states. The main shoulder $(\mathrm{F})$ of the lower peak is attributed to the majority-spin Mn- $e_{g}$ states and the minority-spin $\mathrm{Mn}-t_{2 g}$ states. These two states lie in a same energy region. The $t_{2 g}$ states contribute with the smaller intensity because these orbitals form weak $\pi$ states with the neighboring oxygen atoms. While the $e_{g}$ states form $\sigma$-bonding.

For $\mathrm{CaMnO}_{3}$ the main shoulder (F) of the lower peak has a shoulder (E) in vicinity, towards lower energy. This is shown in the calculated and experimental spectra. The calculated DoS for $\mathrm{CaMnO}_{3}$ exhibits the majority-spin Mn- $e_{g}$ states in two different contributions. The sharp feature at the upper band edge which is attributed to the main shoulder $(\mathrm{F})$ and the broad tail extending towards lower energies which attributes to the lower shoulder (E).

For $\mathrm{PrMnO}_{3}$, the relative weight of the shoulders (E and $\mathrm{F}$ ) which are attributed to the majority-spin $\mathrm{Mn}-e_{g}$ states, decreases. The weight is measured relative to the high energy peaks $(\mathrm{H}$ and $\mathrm{I})$. This weight reduction is attributed to the occupied majority-spin Mn- $e_{g}$ states which are separated from the conduction band. In the ELNES and XANES spectra only the unoccupied orbitals are visible. In $\mathrm{PrMnO}_{3}$ the number of unoccupied majorityspin orbitals is reduced compared to $\mathrm{CaMnO}_{3}$, which is consistent with the corresponding weight reduction.

The experimental ELNES and XANES spectra of $\mathrm{PrMnO}_{3}$ exhibit two shoulders (E and $\mathrm{F}$ ) in the energy range from $1 \mathrm{eV}$ to $3 \mathrm{eV}$, while the calculated spectra exhibits one broad band in this energy range. In the lower part (E) the majority-spin Mn- $e_{g}$ states are contributed, while in the upper part $(\mathrm{F})$ the majority-spin $\mathrm{Mn}-e_{g}$ states contribute along with the small intensity of the minority-spin $\mathrm{Mn}-t_{2 g}$ states. Therefore, the double shoulder ( $\mathrm{E}$ and $\mathrm{F}$ ) of lower peak is attributed to these two features. 


\section{$3.9 \operatorname{Pr}_{1-x} \mathrm{Ca}_{x} \mathrm{MnO}_{3}$ overview}

The electronic, structural and magnetic properties of $\mathrm{Pr}_{1-x} \mathrm{Ca}_{x} \mathrm{MnO}_{3}$ compounds, for $x=0, x=\frac{1}{2}$ and $x=1$ are investigated. All three compounds are insulating at low temperature. Adding electrons to these compounds, forms the localized state which is influenced by cooperative Jahn-Teller distortions. This is in contradiction with the rigidband picture of semiconductor physics. For example adding electrons to silicon, has only a negligible effect on the band structure and electrons in the conduction band are highly conducting.

The hybrid mixing factor is determined by comparing the calculated spectra with the experimental XPS data. This factor is smaller than the value of $a_{x}=0.25$ which is suggested on the basis of perturbation theory [59]. The calculated results are consistent well with the experimental XPS, ELNES, and XANES spectra. Thus, they provide insight into the spectral features.

In $\mathrm{Pr}_{1-x} \mathrm{Ca}_{x} \mathrm{MnO}_{3}$, additional electrons tend to form polarons. The electrons fill states in the conduction band and by the lattice distortion shift them from the conduction band into the valence band. Thus, a band gap opens in the electronic structure. The weight of the filled band increases with increasing the number of electrons. Consequently, the filled band separates from the conduction band and locates about $1 \mathrm{eV}$ to $2 \mathrm{eV}$ above the O-p valence band.

In these compounds there is no metallic phase at low temperatures. This is usually rationalized by a small tolerance factor which makes the Mn-O-Mn angle smaller than $180^{\circ}$. This causes a small hopping amplitude. The present study demonstrates the reliability of the local hybrid functional [63] for strongly correlated systems, such as the $\operatorname{Pr}_{1-x} \mathrm{Ca}_{x} \mathrm{MnO}_{3}$ manganite series.

Now that we have developed a deep understanding for the manganites, further investigations provide a useful insight into the effect of a tilting distortion on the structural, magnetic, and electronic properties. For this purpose, we present $\mathrm{La}_{1-x} \mathrm{Sr}_{x} \mathrm{MnO}_{3}$ calculations to describe the relevant effects.

\subsection{Electronic structure of $\mathrm{La}_{1-x} \mathrm{Sr}_{x} \mathrm{MnO}_{3}$}

Another very important manganite compound is $\mathrm{La}_{1-x} \mathrm{Sr}_{x} \mathrm{MnO}_{3}$, a material that is widely considered as large band-width Mn oxides. In this compound there is a stable FM metallic phase at low temperature. This is usually rationalized as being caused by a tolerance factor, which is about one [3]. For this reason, Mn-O-Mn angle becomes $180^{\circ}$. The hopping amplitude for carriers to move from $\mathrm{Mn}$ to $\mathrm{Mn}$ increases as the angle gets close to $180^{\circ}$. This compound also has a large Curie temperature, as high as $370 \mathrm{~K}$ at intermediate hole doping [3].

In this system, the Mn oxidation state can be controlled from $3+$ to $4+$, and therefore the system can be seen either as hole-doped $\mathrm{LaMnO}_{3}$ or an electron-doped $\mathrm{SrMnO}_{3}$. The 
$\mathrm{LaMnO}_{3}$ and $\mathrm{SrMnO}_{3}$ compounds are antiferromagnetic insulators, while the system becomes a ferromagnetic metal in the intermediate composition range $0.17<x<0.5$. The earliest study of this system was done by Jonker and van Santen [78].

The experimental phase diagram is shown in Fig. 3.19. Although the phase diagram is somewhat schematic and there is still much discussion about the true character of the different states, nevertheless, it is sufficient to show main properties of this compound. In the range of $0.17<x<0.5$, there is a ferromagnetic metallic phase at low temperature. Above the Curie temperature the system is metallic for $x>0.26$, and it turns insulating for $x<0.26$. Metallic phase at low temperature is the interesting magnetotransport properties that we are going to discuss it here.

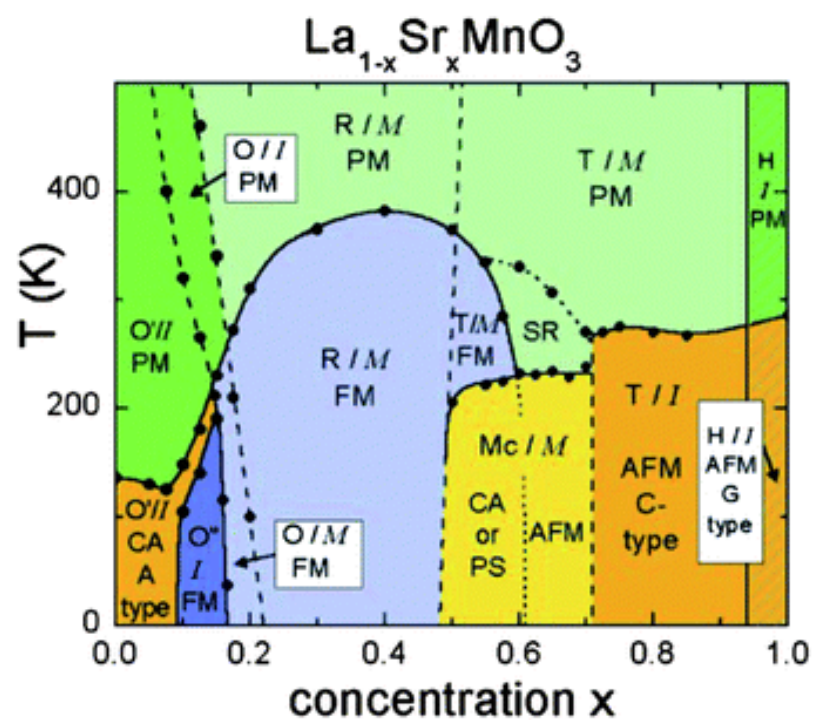

Fig. 3.19: Phase diagram of $\mathrm{La}_{1-x} \mathrm{Sr}_{x} \mathrm{MnO}_{3}$ prepared with Hemberger [119]. PM, PI, FM, FI and CI denote paramagnetic metal, paramagnetic insulator, FM metal, FM insulator, and spin canted insulator states respectively.

At large hole density an antiferromagnetic insulating regime is identified. In the range of $0.7<x<0.9$, the system at low temperature is C-type spin order, but at higher doping level, $0.9<x<1.0$, it is actually G-type. In the other limit of small hole density, $0.0<$ $x<0.1$, an antiferromagnetic insulating regime is stabilized which is actually ferromagnetic within planes and antiferromagnetic between planes (A-type). There is a ferromagnetic insulating regime in the range of $0.1<x<0.17$, which is identified in other manganites as well.

In this section, we will illustrate some aspects of magnetism in manganite using three compounds, cubic $\mathrm{SrMnO}_{3}$, orthorhombic $\mathrm{LaMnO}_{3}$ and the ferromagnetic metal phase of $\mathrm{La}_{\frac{2}{3}} \mathrm{Sr}_{\frac{1}{3}} \mathrm{MnO}_{3}$ in the cubic structure. 


\subsubsection{Stoichiometric $\mathrm{SrMnO}_{3}$}

$\mathrm{SrMnO}_{3}$ has both hexagonal and cubic polymorphs [120], however, in this study the cubic polytype was considered. It is an antiferromagnetic insulating phase with G-type (nearest neighbor anti-ferromagnetism) spin order and Néel temperature of $\mathrm{T}_{N}=240 \mathrm{~K}$ [120]. The related compound, $\mathrm{CaMnO}_{3}$ has an orthorhombic structure, by tilts of the $\mathrm{MnO}_{6}$ octahedra, and the ground state of G-type antiferromagnet with a Néel temperature of $\mathrm{T}_{N}=124 \mathrm{~K}$.

The ions are in the formal oxidation states $\mathrm{Sr}^{2+} \mathrm{Mn}^{4+} \mathrm{O}_{3}^{2-}$. The Calculated DoS of $\mathrm{SrMnO}_{3}$ for A-, B-, C-, and G-type magnetic order is shown in Fig. 3.20. The filled valence band is predominantly $\mathrm{O}-2 p$ character with some contribution of $\mathrm{Mn}-d$ orbitals. The Sr- $d$ states are located 8-10 eV above the valence band. The Mn- $d$ states for the complex properties of manganites lie mostly in between these two features.

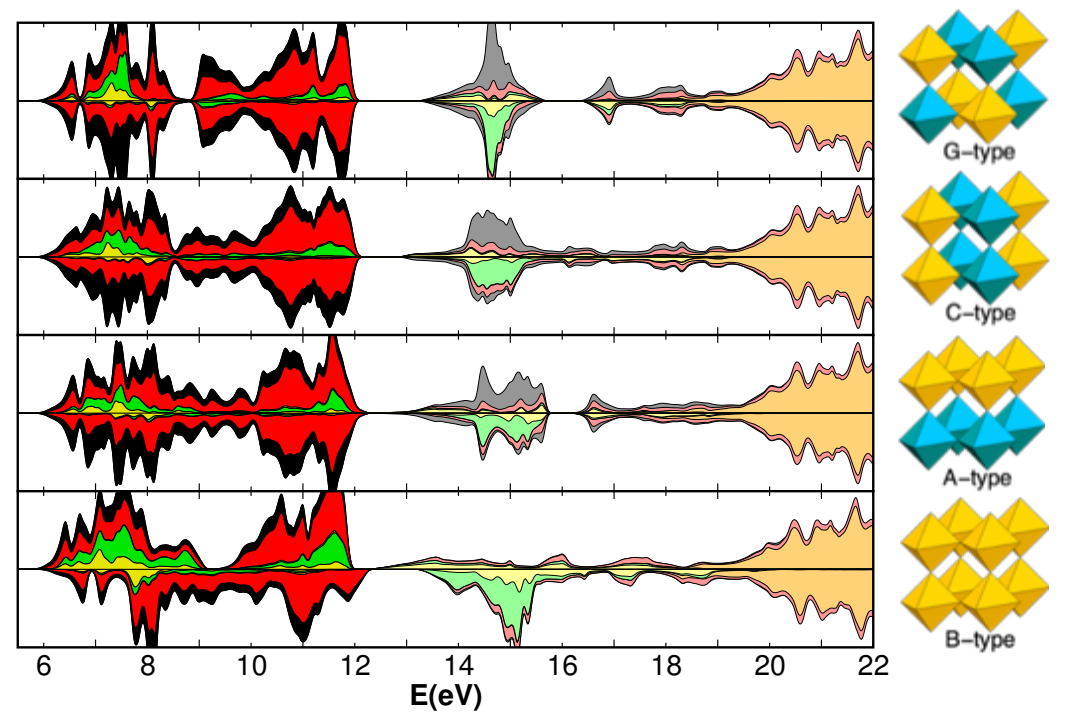

Fig. 3.20: Electronic density of states of cubic perovskite $\mathrm{SrMnO}_{3}$ as obtained with PBE0r. G-type, C-type, A-type, and B-type from top to bottom respectively. The total density of state is black. The area filled in the red is the oxygen contribution, green is the Mn- $t_{2 g}$, yellow area is the Mn- $e_{g}$ and orange area is Sr- $d$ contribution. The empty states are filled with the corresponding lighter color

The Mn-ions have a large magnetic moments, which leads to a Hund's rule splitting between Mn- $d$ levels in the majority and minority spin direction. At the bottom part of oxygen valence band, the bonding states of $\mathrm{Mn}-d$ and $\mathrm{O}-2 p$ are located. The three antibonding $t_{2 g}$ states of the majority spin direction are located in the upper part of the oxygen valence band.Crystal field splitting divides the Mn- $d$ states into $t_{2 g}$ and $e_{g}$ states.

As presented in the Tab. 3.4, our local-hybrid DFT calculations yield an insulator for $\mathrm{SrMnO}_{3}$ with G-type AFM order and band gap about $1.0 \mathrm{eV}$. In contrast to $\mathrm{CaMnO}_{3}$, if ferromagnetic order is imposed, $\mathrm{SrMnO}_{3}$ is predicted to be a metal. This can be seen in Fig. 3.20. Cubic structure of $\mathrm{SrMnO}_{3}$, yields magnetic moments of $2.771 \mu_{B}$ for the 
ferromagnetic (corresponding to $3 \mu_{B}$ per formula unit for the whole cell), and a similar value of $2.766 \mu_{B}$ for the ground state antiferromagnetic structure. This weak dependence of the moment on the ordering means that even at the level of standard band calculations, $\mathrm{SrMnO}_{3}$ behaves like a local moment magnet.

Table 3.4: Magnetic moment $\mu$, number of electrons in the Mn- $d$-shell, calculated relative energies, and fundamental band gaps for different magnetic orders and doping.

\begin{tabular}{cccccc}
\hline \hline \multirow{2}{*}{ Compound } & $\begin{array}{c}\text { Magnetic } \\
\text { order }\end{array}$ & $\mu\left[\mu_{B}\right]$ & $N_{d}$ & Energy(eV) & Band gap (eV) \\
\hline $\mathrm{SrMnO}_{3}$ & $\mathrm{G}$ & 2.760 & 4.801 & -10353.53180041 & 1.23 \\
& $\mathrm{C}$ & 2.750 & 4.804 & -10353.41836590 & 0.62 \\
& A & 2.781 & 4.782 & -10353.23436782 & 0.05 \\
& B & 2.770 & 4.807 & -10353.00264436 & 0.05 \\
\hline $\mathrm{La}_{0.66} \mathrm{Sr}_{0.33} \mathrm{MnO}_{3}$ & $\mathrm{~B}$ & $3.370 / 3.762$ & $4.885 / 4.961$ & -7824.10410494 & - \\
\hline $\mathrm{LaMnO}_{3}$ & $\mathrm{G}$ & 3.648 & 4.910 & -10477.96404283 & 1.23 \\
& $\mathrm{C}$ & 3.694 & 4.911 & -10477.97931637 & 0.61 \\
& A & 3.771 & 4.912 & -10478.24312432 & 1.12 \\
& B & 3.819 & 4.910 & -10478.19781528 & - \\
\hline \hline
\end{tabular}

From an experimental point of view $\mathrm{SrMnO}_{3}$ is clearly insulating both above and below $\mathrm{T}_{N}$ [121]. Our calculations yield an AF insulating state, which is more stable than ferromagnetic one. Also band picture implies that the conductivity should increase when the AF order is destroyed above $\mathrm{T}_{N}$ since it relates to the specific magnetic order. Essentially, the description of the electronic structure and the true nature of the gap is slightly different. This is because there is not a good description of band gap and neither a clear experiment showing the magnitude of the gap in the paramagnetic phase for this material. Importantly very tiny dopings produce metallic conduction near $\mathrm{T}_{N}$ suggesting that the material is almost metallic [3].

Let's compare these results to our previous calculations. $\mathrm{CaMnO}_{3}$ is a much more clear case. The principal difference between the two materials is structural, i.e. that in $\mathrm{CaMnO}_{3}$ the $\mathrm{MnO}_{6}$ octahedra are rotated yielding an orthorhombic structure. Such a structural distortion will generally narrow the bands (especially the $e_{g}$ band) in a perovskite, favoring a correlated state. Interestingly, in $\mathrm{FM} \mathrm{CaMnO}_{3}$ we have band gap about $1.3 \mathrm{eV}$ which is large enough to be an insulating while here in $\mathrm{SrMnO}_{3}$ we don't have any band gap and it behaves as a metallic phase. This property is somewhat puzzling since we have the same story for G-type AFM in the description of band structure.

In the $\mathrm{SrMnO}_{3}$ (Fig. 3.20), from electron counting, there are three $d$-electrons in $\mathrm{Mn}$ ${ }^{4+}$, and so this should normally lead to a fully occupied majority spin $t_{2 g}$ manifold. The 
main O- $2 p$ bands occur in the energy range from $-6 \mathrm{eV}$ to $\sim 0 \mathrm{eV}$. Focusing on the G-type $\mathrm{SrMnO}_{3}$ (top figure), the minority spin shows a crystal field splitting of $\sim 2 \mathrm{eV}$ between well defined $t_{2 g}$ and $e_{g}$ manifolds. In the majority spin the occupied $t_{2 g}$ manifold overlaps the top of the $\mathrm{O}-2 p$ bands due to the exchange splitting, and $\mathrm{Mn} e_{g}$ character at the bottom of the valence bands. The hybridization in $\mathrm{SrMnO}_{3}$ is very spin dependent, with clearly stronger hybridization for the majority spin $e_{g}$ than for the minority spin. This is important for the metallicity of the ferromagnetic ordering.

\subsubsection{Stoichiometric $\mathrm{LaMnO}_{3}$}

$\mathrm{LaMnO}_{3}$ and $\mathrm{PrMnO}_{3}$ are similar in structure. The crystal structure of $\mathrm{LaMnO}_{3}$ can be described as a perovskite with tilting that Mn-O-Mn angles become less than $180^{\circ}$, and Jahn-Teller distortion of the octahedra, with one long Mn-O bond and two short bonds, types of distortion from a cubic structure. This compound shows similar properties as $\mathrm{PrMnO}_{3}$. The main difference is due to the larger ionic radius of La ion compared with Pr. The larger ionic radius of La causes less amount of the tilting of the oxygen octahedra in orthorhombic Pbnm crystal structure. This effect induces the larger effective $e_{g}-e_{g}$ hopping between $\mathrm{Mn}$ atoms and smaller band width.

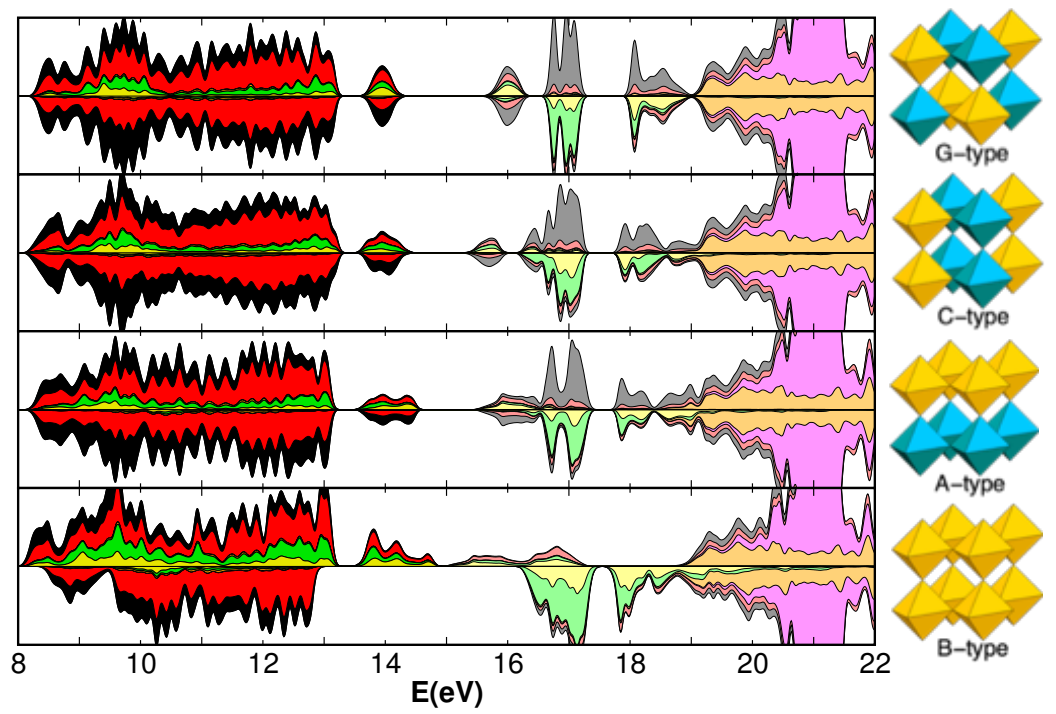

Fig. 3.21: Electronic density of states of orthorombic perovskite $\mathrm{LaMnO}_{3}$ as obtained with PBE0r. G-type, C-type, A-type, and B-type from top to bottom respectively. The total density of state is black. The area filled in the red is the oxygen contribution, green is the Mn- $t_{2 g}$, yellow area is the Mn- $e_{g}$, magenta area is La- $f$ and orange area is La- $d$ contribution. The empty states are filled with the corresponding lighter color.

The density of states for the ground state magnetic structure as obtained within the PBE0r is shown in Fig. 3.21. A small band gap insulator is predicted (band gap about $1.12 \mathrm{eV}$ ). However, the electronic density of states is qualitatively different from $\mathrm{SrMnO}_{3}$. 
Calculations were carried out for the G-, C-, A-type AF and B-type FM using the orthorhombic structure (Pbnm space group), taken from earlier experimental report [122].

Here, the Mn-O bond and the degree of covalency are certainly less strong than $\mathrm{SrMnO}_{3}$. It is worth mentioning that the Mn- $3 d$ and the O- $2 p$ hybridization is evaluated spin dependent. The magnetic moment of $\mathrm{Mn}$ atom in $\mathrm{LaMnO}_{3}$ compound is obtained $3.86 \mu_{B}$. This shows a strong moment of $\mathrm{Mn}$ in $\mathrm{LaMnO}_{3}$ and weak hybridization compared to $\mathrm{SrMnO}_{3}$ because magnetic moment depend on the degrees of overlapping of electronic shells.

\subsubsection{Stoichiometric $\mathrm{La}_{0.66} \mathrm{Sr}_{0.33} \mathrm{MnO}_{3}$}

Half-metallic ferromagnets are systems where up spins have a partially occupied band, while the down spins have filled bands by a gap. Among the manganites, $\mathrm{La}_{1-x} \mathrm{Sr}_{x} \mathrm{MnO}_{3}$ at low temperature may have half-metallic character (is still under debates). In case of half doped system, we simulated the structure by a 15 -atoms unit cell which can be schematically represented as a [001]-ordered $\left(\mathrm{SrMnO}_{3}\right)_{1} /\left(\mathrm{LaMnO}_{3}\right)_{2}$ superlattice. $\mathrm{MnO}_{6}$ octahedral tilting also neglected.

In our calculations, the three Mn atoms in the unit cell were aligned ferromagnetically. The calculated DoS is shown in Fig. 3.22. It is presented that a gap exists in the spin-down spectrum with $p$ - $d$ orbital character, while the spin-up band has metallic behavior. This DoS shows that the main part of the majority $\mathrm{Mn}-t_{2 g}$ states are at the bottom part of the O- $p$ band. The empty states of La- $f$ are significantly in the conduction band and does not participate in the valence band. Additionally, the majority $e_{g}$ states broadens about $6 \mathrm{eV}$ in the spin up band. This picture of band structure is in the agreement with experimental photoemission techniques [3]. They have shown that the conduction electrons at the Fermi level are $90 \%$ polarized at low temperature in thin films of $\mathrm{La}_{0.66} \mathrm{Sr}_{0.33} \mathrm{MnO}_{3}$. They have claimed also at low temperature a gap exists in the spin-down spectrum, while the spin-up band has metallic behavior.

\subsection{4 $\mathrm{La}_{1-x} \mathrm{Sr}_{x} \mathrm{MnO}_{3}$ Overview}

All two compounds investigated, with $x=0$ and $x=1$, are insulating at low temperature but half-doped system is a metallic phase. They all have a perovskite-based crystal structure, but for $x=0$ orthorhombic one is more favorable and for Sr-doped systems, cubic perovskite is appeared. $\mathrm{La}_{1-x} \mathrm{Sr}_{x} \mathrm{MnO}_{3}$ has a rich electronic phase diagram, including a doping-dependent metal-insulator transition, paramagnetism and ferromagnetism.

In our calculations, $\mathrm{La}_{1-x} \mathrm{Sr}_{x} \mathrm{MnO}_{3}$ is one of the perovskite manganites that shows an observed half-metal for compositions around $x=0.33$. It displays a colossal magnetoresistance effect. Above its Curie temperature (about 350K) Jahn-Teller polarons are formed; the material's ability to conduct electricity is dependent on the presence of the polarons [3]. 


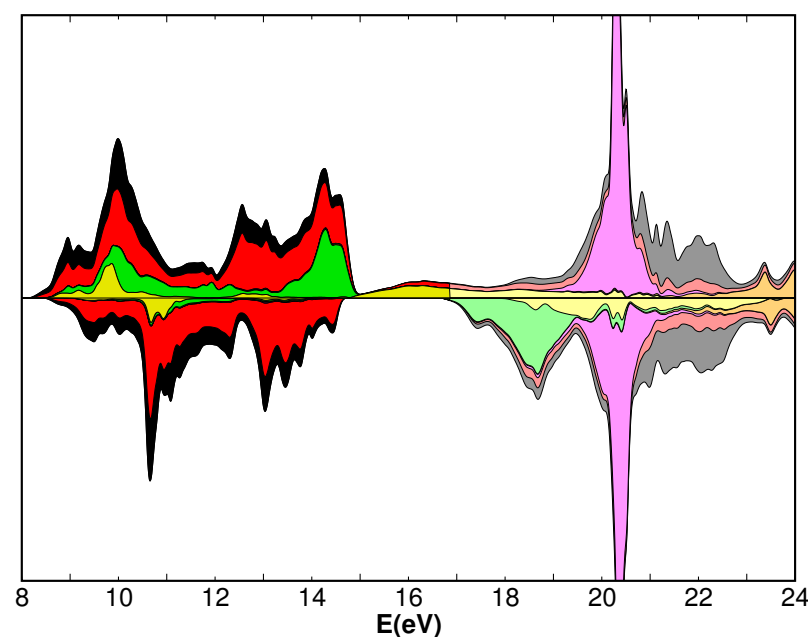

Fig. 3.22: Electronic density of states of cubic perovskite $\mathrm{La}_{0.66} \mathrm{Sr}_{0.33} \mathrm{MnO}_{3}$ as obtained with PBE0r. The total density of state is black. The area filled in the red is the oxygen contribution, green is the $\mathrm{Mn}-t_{2 g}$, yellow area is the Mn- $e_{g}$, magenta area is La- $f$ contribution and orange area is the Sr- $d$. The empty states are filled with the corresponding lighter color.

\subsection{Summary}

First-principles calculations using local hybrid functionals for $\operatorname{Pr}_{1-x} \mathrm{Ca}_{x} \mathrm{MnO}_{3}$ have been performed for the dopings $x=0,1 / 2,1$. In contrast to typical hybrid-functional calculations, the additional computational cost for local hybrid functional calculations over conventional density functional calculations is negligible, and in particular so for large systems.

The hybrid mixing factor has been determined by comparing with experimental XPS data. We find this factor to be substantially smaller than the value of 0.25 suggested on the basis of perturbation theory [61]. Furthermore a careful analysis of the experimental spectra indicates that, that methods with adjustable Fock term may need to reduce its contribution further.

The first-principle results compare well with experimental spectra such as XPS, photoconductivity measurements ELNES and XANES. Thus they provide insight into the nature of the spectral features. We find that the NIR absorption feature has a different origin for the the undoped and half-doped material. The calculated EELS spectra allow to assign the features in the ELNES and XANES spectra. Nevertheless, our calculations seem to overestimate band gaps still by about $0.2 \mathrm{eV}$.

Furthermore, local hybrid calculations for $\mathrm{La}_{1-x} \mathrm{Sr}_{x} \mathrm{MnO}_{3}$ have been performed for the dopings $x=0,1 / 3,1$. The effects of magnetic ordering and tilting angle reveal that the larger ionic radius of La ion compared with Pr causes larger effective hopping between Mn atoms. 


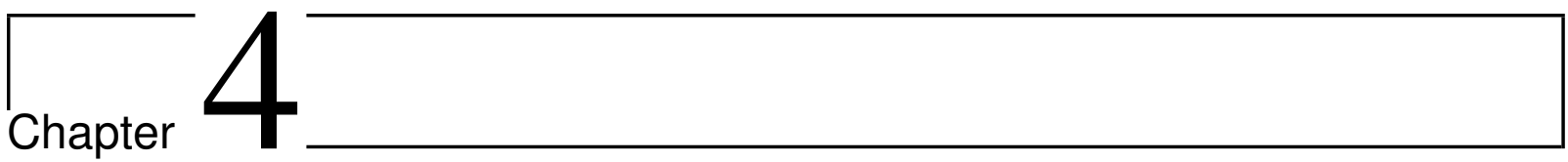

\section{Water splitting}

Perovskite oxides are nominated as catalysts for the electrolysis of water in the production of efficient and sustainable fuel. However, understanding of the mechanism for water electrolysis on perovskite surfaces, play a dominant role for design process of active catalysts. In previous chapter, we presented a series of manganites where constructed by $\mathrm{Ca}^{2+}$ substitution into $\mathrm{PrMnO}_{3}$. Here, we attempt to rationalize the high activities of $\mathrm{Pr}_{1-x} \mathrm{Ca}_{x} \mathrm{MnO}_{3}$ in the contact with water through the electronic structure using density functional theory (DFT). We show DFT descriptors of oxygen evolution reaction. The reaction between two water molecules in the presence of a hole will be discussed in details and later on, this mechanism will be applied on the surface to find out an oxygen evolution reaction path way.

\subsection{Catalysis for sustainable energy}

Using a sustainable energy source such as solar energy is getting more attention for the production of useful fuels. The first step for solar energy conversion technologies is the absorption of photon [123]. However, electronic excitations are short-lived. To solve this challenge, a mechanism for longer-term energy storage is required. The most efficient and popular way is to store the solar energy in chemical bonds [124, 125]. Then, this technique can be used to split water and finally, produce oxygen and hydrogen gas as a fuel. This reaction is considered as shown in a following equation,

$$
2 \mathrm{H}_{2} \mathrm{O} \rightarrow \mathrm{O}_{2}+2 \mathrm{H}_{2} \quad E^{0}=1.23 \mathrm{~V}
$$

where $E^{0}$ is the required energy respect to the standard hydrogen electrode (SHE). At first glance, two molecules of water come close and split into four proton and a molecule of $\mathrm{O}_{2}$. Then four electrons release at a potential of $1.23 \mathrm{~V}$ per electron with reference to the SHE. On the other hand, two protons and two electrons combine to form a molecule of 
$\mathrm{H}_{2}$. Nevertheless, the full catalytic cycle, involves several intermediates that dictate overall catalytic activity.

The mechanism of $\mathrm{O}-\mathrm{O}$ bond formation and releasing of $\mathrm{O}_{2}$, is one of the factors to determine the catalyst's efficiency. The acid-base mechanism [126] proposed that O-O bond formation is based on the nucleophilic attack on an electrophilic metal oxo species. By contrast, the direct coupling mechanism [126] proposed that two high-valent metal oxo species on the surface come together to form $\mathrm{O}_{2}$. The transition-metal system is responsible for proton coupled electron-transfer (PCET) events. Additionally, the aqueous environment play a dominant role in providing the proper electrostatic environment and also as a reactant itself. Ultimately, releasing of $\mathrm{O}_{2}$ from catalyst is done by water.

Artificial water splitting has received a wide attention because it directly governs rechargeability of batteries [127] and regenerate fuel cells [128]. Effective catalysts are made from transition-metal complexes [129], cobalt oxides [130], and perovskite materials [17] under several different mechanisms.

Recently, complex oxide with perovskite structure have shown high oxygen evolution reaction (OER) activities compared to precious metal based catalysts such as $\mathrm{RuO}_{2}$ and $\mathrm{IrO}_{2}[129,131]$. However, a fundamental understanding of the OER mechanism for different perovskites is unknown. Experimental results [132] on highly active perovskites such as $\mathrm{La}_{1-x} \mathrm{Sr}_{x} \mathrm{CoO}_{3-\delta}$ and $\mathrm{SrCoO}_{3-\delta}$ have shown that the lattice oxygen from the perovskites involved during OER. In addition, DFT studies [129, 131, 133, 134] have shown that the conventional acid-base mechanism $\left({ }^{*} \mathrm{OH} \rightarrow{ }^{*} \mathrm{O} \rightarrow{ }^{*} \mathrm{OOH} \rightarrow \mathrm{O}_{2}(\mathrm{~g})\right)$ is favorable for strongly binding perovskites such as $\mathrm{LaCoO}_{3}$, while the lattice oxygen mechanism (LOM), in which the surface lattice oxygen $\left(\mathrm{O}_{\text {surf }}\right)$ participates in the reaction, is favorable for weakly binding perovskites such as $\mathrm{LaCuO}_{3}$.

DFT calculations can be used to make useful prediction about the mechanism of water splitting and also about the thermodynamic stability of intermediate reactions. In the following section, computational details is described. Next, a theoretical study is carried out to investigate the molecular and electronic structures of the water dimer radical cations. The purpose of this study is to characterize the structures of the stationary points on the electronic doublet potential energy surface (PES) and to examine the dissociation pathways of ionized water dimers. These results are essential to analyze and understand the mechanism of proton transfer into the dimeric water and finally water splitting process.

\subsection{Computational details}

All calculations for molecules were performed using the PBE0r local hybrid functional. In the PBE0r functional, the parameter $a_{x}$ controlling the Fock admixture of the hybrid functional can be chosen individually. we used $a_{x}=0.1$ for $\mathrm{O}$ and $\mathrm{H}$. The plane wave cutoff for the auxiliary wave function is $40 \mathrm{Ry}$ and $80 \mathrm{Ry}$ for the density. we specified a face-centered cubic unit cell with a lattice constant of $10.36 \AA$. In order to avoid an overlap of the wave functions with those from a periodic images, keeping a large distance between 
periodic images is not sufficient. The electrostatic interaction is long ranged. Here we use a technique of removing the electrostatic interaction of periodic images [135]. This technique calculates the electrostatic interaction between the periodic images and subtracts its energy from the Hamiltonian.

A k-point grid of $2 \times 2 \times 2$ points is chosen. There are $8 \mathrm{k}$-points in total. We used spin polarized calculations for ionized system. For the augmentation, a $s^{1} p^{1} d^{1}$ set of projector functions was used for $\mathrm{O}$ atom and a $s^{1} p^{1}$ set for $\mathrm{H}$ atom. The superscripts denote the number of projector functions angular momentum channels. All atomic positions are optimized without symmetry constraints.

\subsection{Water dimer radical cation}

Water can be decomposed when it brings into the contact with surfaces. The products may contain hydrogen atom $(\mathrm{H})$, hydroxide radical $(\mathrm{OH})$, oxygen anions $\left(\mathrm{O}^{-}\right)$, and water cations $\left(\mathrm{H}_{2} \mathrm{O}^{+}\right)$. These reactions lead to the formation of ionized water living in about several tens femtoseconds and hydrated electron, followed by the generation of hydronium $\left(\mathrm{H}_{3} \mathrm{O}^{+}\right)$and $\mathrm{OH}$ radicals through proton transfer process. Chemical dynamics of water splitting can be understood through the smallest system such as water dimer radical cation $\left(\mathrm{H}_{2} \mathrm{O}\right)_{2}^{+}$. Here we start with the water molecule and we try to analyze the particular reaction for the O-O bond formation.

\subsubsection{Molecular orbitals for water molecule}

The calculated molecular orbitals of the isolated water molecule is shown in Fig. 4.1. The lowest energy orbital $\left(2 a_{1}\right)^{2}$ is contributed from the $1 s$ orbitals of the hydrogen atoms and the $2 s$ orbital of the oxygen atom. On top of that, $\left(1 b_{2}\right)^{2}$ orbital is contributed from $\mathrm{O}-2 p_{x}$ and $\mathrm{H}-1 s$. After that, $\left(3 a_{1}\right)^{2}$ has a contribution of $\mathrm{O}-2 p_{y}$ and $\mathrm{H}-1 s$. The highest energy occupied orbital, $\left(1 b_{1}\right)^{2}$ is predominantly $\mathrm{O}-p_{z}$ with no contribution of $\mathrm{H}-1 s$ orbital. The highest occupied orbital is non-bonding and highly localized on the oxygen atom without $s p^{3}$ hybridization characteristic. The three orbitals $2 a_{1}, 1 b_{2}$ and $3 a_{1}$ are $\mathrm{O}-\mathrm{H}$ bonding orbitals. The two lowest unoccupied molecular orbitals $4 a_{1}$ (LUMO) and $2 b_{2}$ contribute to the $\mathrm{O}-\mathrm{H}$ antibonds.

The bond length of $0.987 \AA$ and the bond angle of $104.8^{\circ}$ for water molecule extracted from our PBE0r calculations. The experimental values for gaseous water molecule are $0.95718 \AA$ for O-H bond length, and $104.474^{\circ}$ for H-O-H bond angle [136]. Our calculated $\mathrm{O}-\mathrm{H}$ bond-length is about $3 \%$ larger than the actual value and, the bond angle is in a good agreement with the experimental value. The calculated band gap is about $5.61 \mathrm{eV}$.

Santra et al. [137] investigated the deformation energies calculatedd with PBE, BLYP, and PBE0 functionals. They have shown that the symmetric stretching of the $\mathrm{O}-\mathrm{H}$ bonds of a water monomer is understimated by PBE and BLYP while it is accurate given by PBE0. Leung and Rempe [138] have considered systematic series of simulations for the 


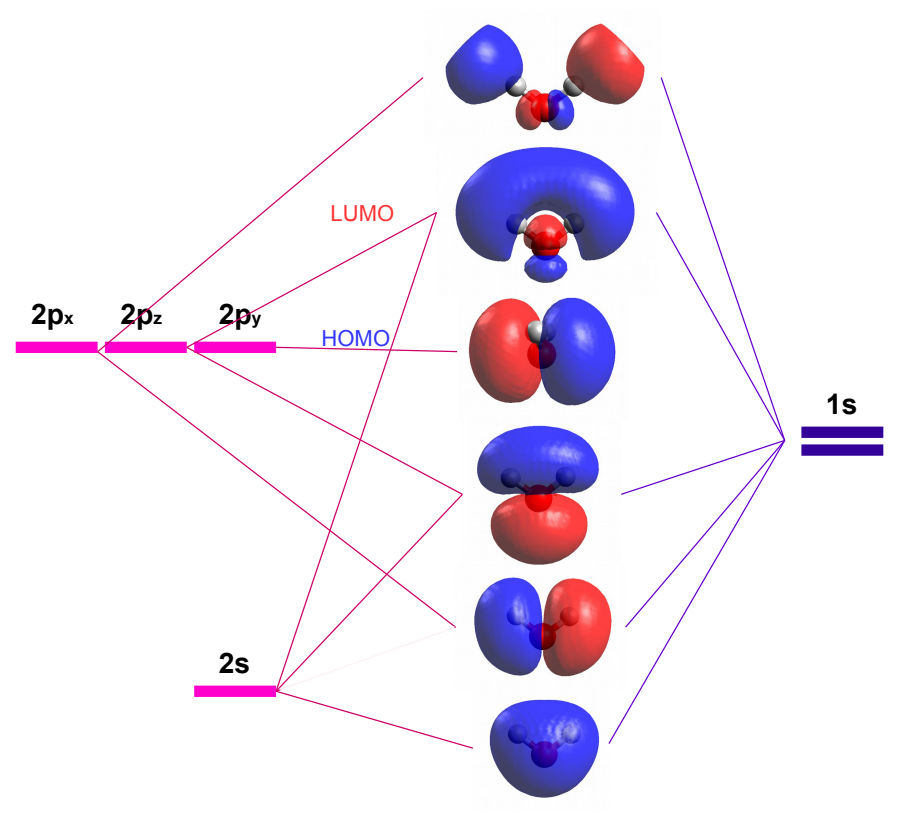

Fig. 4.1: The four occupied and the lowest two unoccupied molecular orbitals of the isolated water molecule $\left(2 a_{1}\right)^{2}\left(1 b_{2}\right)^{2}\left(3 a_{1}\right)^{2}\left(1 b_{1}\right)^{2}\left(4 a_{1}\right)^{0}\left(2 b_{2}\right)^{0}$. The orbital representations consider the respective sign of wave function as indicated with the blue $(-)$ and red $(+)$ color.

length of the $\mathrm{O}-\mathrm{H}$ bonds.

Now, we turn our attention to the water dimer by adding a water molecule next to the previous one.

\subsubsection{Plausible structure for water dimer}

The optimized structures of the neutral water dimer are shown in Fig. 4.2. The three configurations are classified in the following three structures: linear water dimer with a $C_{S}$ symmetry, cyclic dimer water with a $C_{2 h}$ symmetry, and bifurcated water dimer with a $C_{2 v}$ symmetry molecular geometry. The water dimer has $C_{S}$ symmetry molecular geometry in the equilibrium. At short distance between $\mathrm{O}$ atoms $(<2.66)$, the cyclic water dimer with a doubly-hydrogen bonded structure has been found to be more stable than the $C_{S}$ dimer $[136,139]$. The $C_{2 v}$ dimer is normally about $31 \mathrm{meV}$ less stable than the $C_{S}$ dimer [140,141]. It is a transition structure for the interchange between the molecules being the hydrogen donor or acceptor.

The related total energies for the different structures are listed in Tab. 4.1. Our PBE0r calculations show that the linear water dimer has the lowest total energy. The linear water 


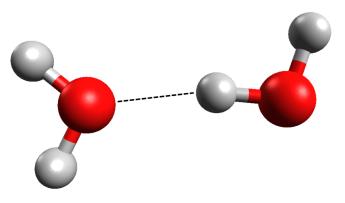

(a)

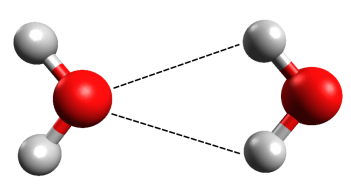

(b)

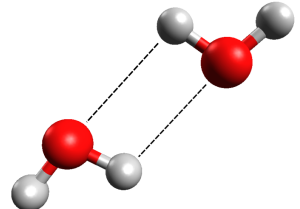

(c)

Fig. 4.2: Three possible structures of water dimer: (a) linear, (b) bifurcated, and (c) cyclic water dimer. The red spheres represent the formal $\mathrm{O}$ ions and the gray spheres show the formal $\mathrm{H}$ ions. Dashed lines stand for hydrogen bond.

dimer energetically is so close to the cyclic one. The highest energy is calculated for the bifurcated water dimer. The calculated band gap for the linear water dimer is smaller than two other structures.

The neutral and non-spin-polarized water dimer has a hydrogen bond between $\mathrm{H}$ and $\mathrm{O}$. The equilibrium structure is linear water dimer. The bond length between $\mathrm{H}$ and $\mathrm{O}$ that forms a hydrogen bond, is $1.984 \AA$. The next step is to find out where the molecular orbitals are responsible for the hydrogen bond. The orbital interaction diagram for the linear water dimer is shown in Fig. 4.3. The two orbitals show overlap across the hydrogen bond, supporting the partial covalent nature of the hydrogen bond. In the higher energies, the corresponding antibondings interaction are presented.

The HOMO of the system is the $p_{z}$ orbital perpendicular to the molecular plane at $\mathrm{O}$ site. The HOMO is located at the water molecule which oxygen atom does not receive a hydrogen bond. Creating a hole can be assumed that the hole is at the former HOMO. The HOMO-LUMO gap is $5.1458 \mathrm{eV}$.

\subsubsection{Adding a hole: proton transfer and hemibonded structure}

By removing an electron from the water dimer, the radical cation is formed. The geometry of an ionized system may be different from the neutral one. The ionization energy can refer to the vertical ionization energy or to the adiabatic ionization energy. In the case of the same geometry as the neutral system, vertical ionization energy is defined, while adiabatic

Table 4.1: Calculated total energies of the three water dimer structures and the related band gaps.

\begin{tabular}{cccc}
\hline \hline & Linear & Bifurcated & Cyclic \\
\hline Binding energy $(\mathrm{eV})$ & -1.7977 & -1.7694 & -1.7976 \\
Gap $(\mathrm{eV})$ & 5.1458 & 5.4024 & 5.9562 \\
\hline \hline
\end{tabular}




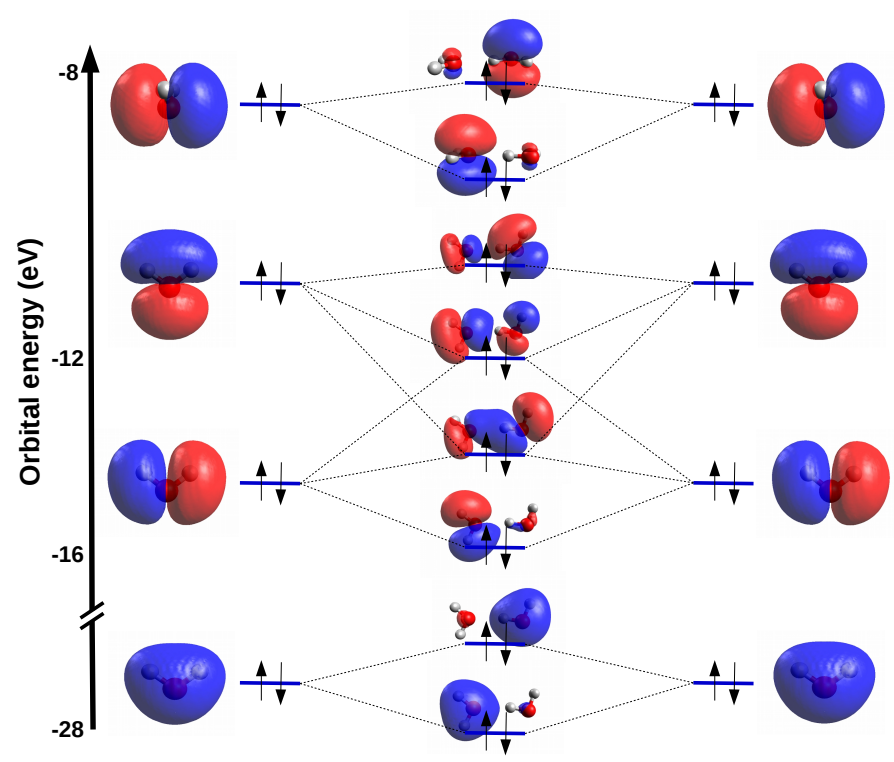

Fig. 4.3: The orbital interaction diagram of $\left(\mathrm{H}_{2} \mathrm{O}\right)_{2}$. The orbital representations consider the respective sign of wave function as indicated with the blue $(-)$ and red $(+)$ color.

ionization is refer to the ion in its lowest energy. For the linear water dimer the vertical ionization energy is $12.85 \mathrm{eV}$. The band gap decreases to $0.54 \mathrm{eV}$. It should be noted that the radical cation system has a total spin of $0.5 \hbar$.

After relaxation, two minimum structures of the water dimer radical cation have been identified: the proton transferred and the hemibonded structure. These two structures are shown in Fig. 4.4. The previous DFT calculations have shown that many exchangecorrelation functionals fail to predict the presence of hemibonding interaction [139]. The hemibonding interaction highlights the serious self-interaction errors in the density functional approximations. Hybrid functionals with larger fraction of the exact Hartree-Fock exchange are suggested for more accurate results in this structure [139]. In the present work, local hybrid functionals are performed to ensure the self-interaction cancellation.

Movement of the atoms into the new equilibrium situations are calculated. The previously formed hydrogen bond is broken. In the hemibonded structure, the oxygen atoms now point directly to each other while in the proton transferred structure a hydronium points to a hydroxide by a new hydrogen bond. The O-O bond length are about $2.1 \AA$ and $2.5 \AA$ for hemibonded and proton-transfer structure respectively. Before going into the details about how these two ground states are related to each other, we review the orbital representation of these two structures.

The orbital interaction diagram of hemibonded structure is shown in Fig. 4.5. The rotation of the water molecule has made it possible to overlap the $p_{z}$ orbitals of both water molecules. The O-O distance is decreased. The stabilization energy by the rotation is 0.89 $\mathrm{eV}$. The effect of the rotation on the highest occupied orbital will be discussed.

The highest occupied state is the $\mathrm{O}-\mathrm{O}$ antibonding of the $p_{z}$ orbitals. It should be 


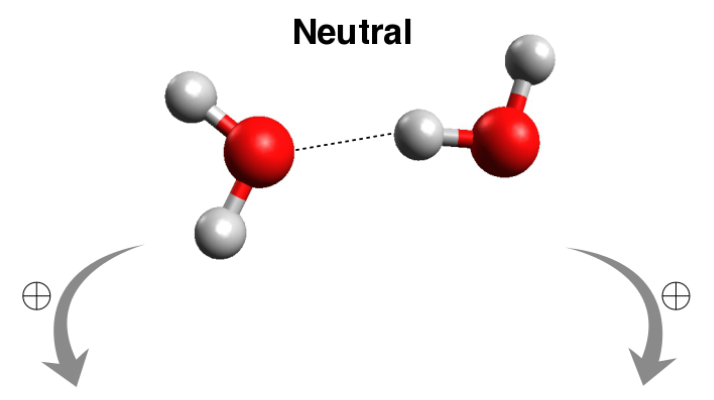

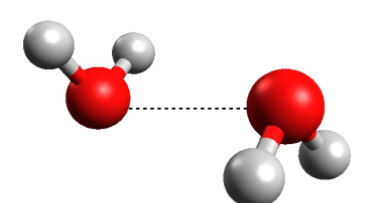

Hemibonded

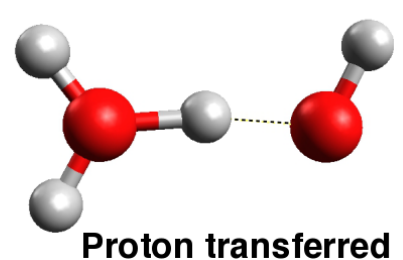

Fig. 4.4: The equilibrium structures of $\left(\mathrm{H}_{4} \mathrm{O}_{2}\right)$ and $\left(\mathrm{H}_{4} \mathrm{O}_{2}\right)^{+}$. The water dimer radical cation has two structures: proton transferred (right) and hemibonded structure (left). The red spheres represent the formal $\mathrm{O}$ ions and the gray spheres show the formal $\mathrm{H}$ ions. Adding a hole is represented by $\oplus$.

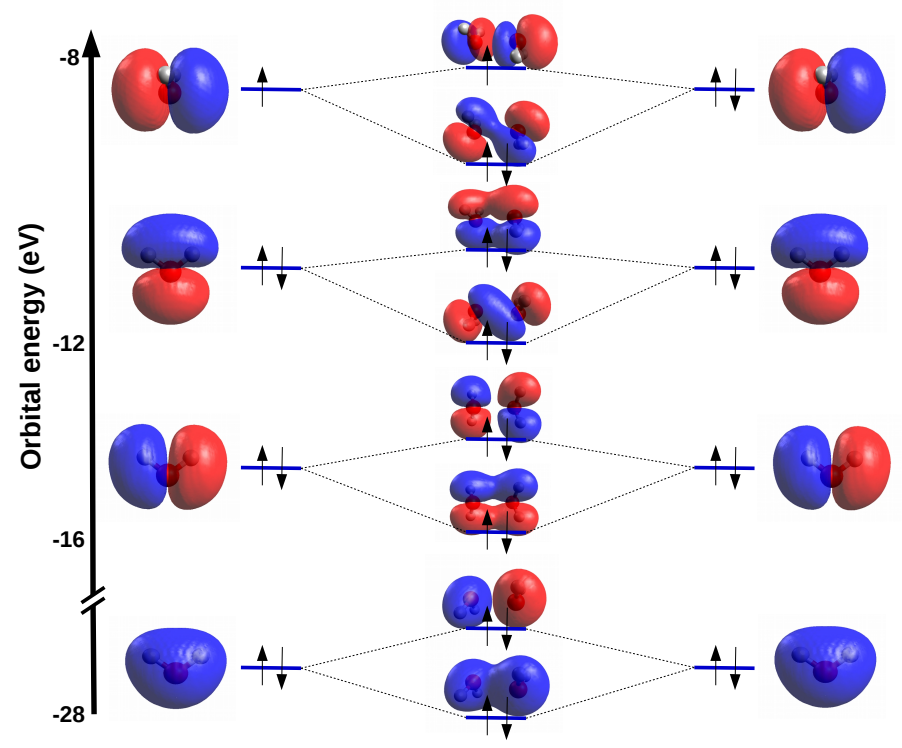

Fig. 4.5: The orbital interaction diagram of hemibonded structure for $\left(\mathrm{H}_{2} \mathrm{O}\right)_{2}^{+}$.

noted that the corresponding $\mathrm{O}-\mathrm{O}$ bonding effects are stabilized. Due to the rotation, the $p_{z}$ orbitals now point directly to each other. The $p_{z}$ orbitals in the HOMO have a same contribution. The HOMO band is the highest occupied orbital with spin up. The band gap is $2.36 \mathrm{eV}$ which is increased compared to the non-relaxed system. 
In addition to the band gap formation, the rotation provides balanced atomic charges. Before the rotation, the hole was mostly located at one of the oxygen atoms. After rotation this charge is localized between the two oxygen atoms. This contributes to the stabilization of the rotated state.

The rotation changes the quality of the bonding. The hole is placed in the highest occupied level, supporting the antibonding O-O bond. Although our calculations apply strictly only to isolated water dimer in the gas phase, they may nevertheless provide information that will be helpful in the interpretation of solution and solid phase experiment.

The orbital interaction diagram of the proton transferred structure is shown in Fig. 4.6. The hole is located on the $\mathrm{H}_{2} \mathrm{O}$ and attract proton to make a hydronium $\mathrm{H}_{3} \mathrm{O}^{+}$.

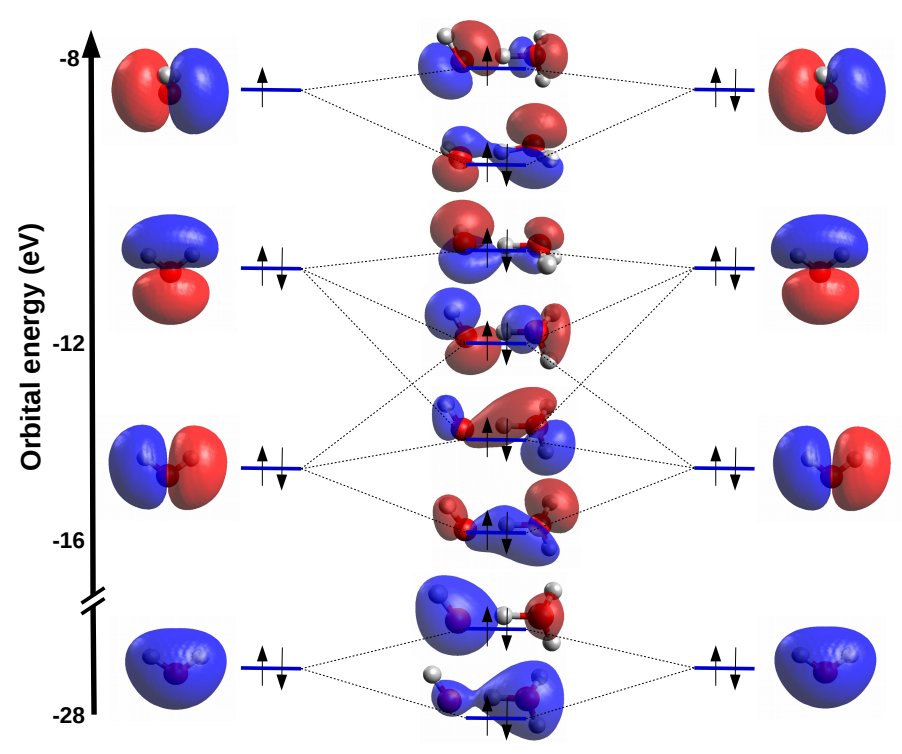

Fig. 4.6: The orbital interaction diagram of PT structure for $\left(\mathrm{H}_{2} \mathrm{O}\right)_{2}^{+}$.

Two molecular orbitals cross the region between the two water monomers. These two crossing molecular orbitals are mainly composed of the O- $2 p$ orbital and H- $1 s$ orbital. The highest occupied state is the $\mathrm{O}-\mathrm{O}$ antibonding of the $p_{z}$ orbitals. The $p_{z}$ orbitals in the HOMO do not have a same contribution. The $p_{z}$ orbital of the hydroxide is more pronounced in the HOMO band compared to the hydronium $p_{z}$ orbital. The HOMO band is the highest occupied orbital with spin up. The band gap is $2.75 \mathrm{eV}$ which is increased compared to the non-relaxed system.

Based on the PBE0r-DFT calculations, two minima have been characterized. The total energies and band gaps for these two minima are listed in Tab. 4.2. The energy difference between these two structures is about $0.3 \mathrm{eV}$. The ground state of the water dimer radical cation has been calculated to be a hemibonded structure. The O-O distance in the hemibonded structure decreases compare to the proton transferred structure by $0.34 \AA$. 
Table 4.2: Calculated total energies and band gaps for the two different structures of $\left(\mathrm{H}_{4} \mathrm{O}_{2}\right)^{+}$structure.

\begin{tabular}{ccc}
\hline \hline & Hemibonded structure & Proton transferred structure \\
\hline Energy $(\mathrm{eV})$ & 0.000 & 0.289 \\
Gap $(\mathrm{eV})$ & 2.7548 & 2.3613 \\
O-O distance $(\AA)$ & 2.164 & 2.508 \\
\hline \hline
\end{tabular}

\subsubsection{Adding second hole: proton transfer vs hemibonded struc- ture}

By removing an electron from the previous structures we can get some insight to the O-O bond formation. The two structures of the water dimer radical cation are investigated by adding two holes into the systems. In the proton transferred structure the hydrogen bond between the hydroxide and $\mathrm{H}_{3} \mathrm{O}^{+}$breaks down and the O-O distance is found to be $7 \AA$. In the hemibonded structure second hole is located between the two oxygen atoms and make $\mathrm{O}-\mathrm{O}$ bond distance about $1.5 \AA$. The hemibonded structure with two holes is shown In Fig. 4.7.

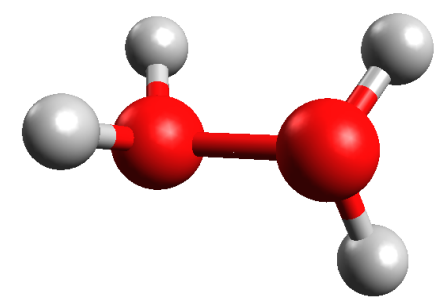

Fig. 4.7: Structure of $\left(\mathrm{H}_{4} \mathrm{O}_{2}\right)^{2+}$. The O-O bond formation is represented by a solid red line.

The orbital interaction diagram of the $\left(\mathrm{H}_{4} \mathrm{O}_{2}\right)^{2+}$ structure is shown in Fig. 4.8. The second hole is located on the singly occupied HOMO band and makes it empty. By comparing the orbital interactions in Fig. 4.5 and Fig. 4.8, it can be seen that the antibonding state between the two oxygen atoms is empty and located in the LUMO band. The band gap is $5.9 \mathrm{eV}$. When the $p_{z}$ antibonding combination is pushed up to the Fermi level, interactions is attractive.

\subsubsection{Transition state for water dimer radical cation}

For the water dimer radical cation $\left(\mathrm{H}_{4} \mathrm{O}_{2}\right)^{+}$, there are fourteen minima [139] which three of them available in Tab. 4.3. The proton transferred structures (1) with a bridging hydrogen which is so-called group $\mathrm{A}$ and they are shown as $\mathrm{H}_{2} \mathrm{O}-\mathrm{H}^{+} \ldots$ OH. Group $\mathrm{B}\left(\mathrm{H}_{3} \mathrm{O}^{+} \ldots\right.$ $\mathrm{OH}$ ) proton transferred structures without the hydrogen bridging. And finally, group $\mathrm{C}$ 


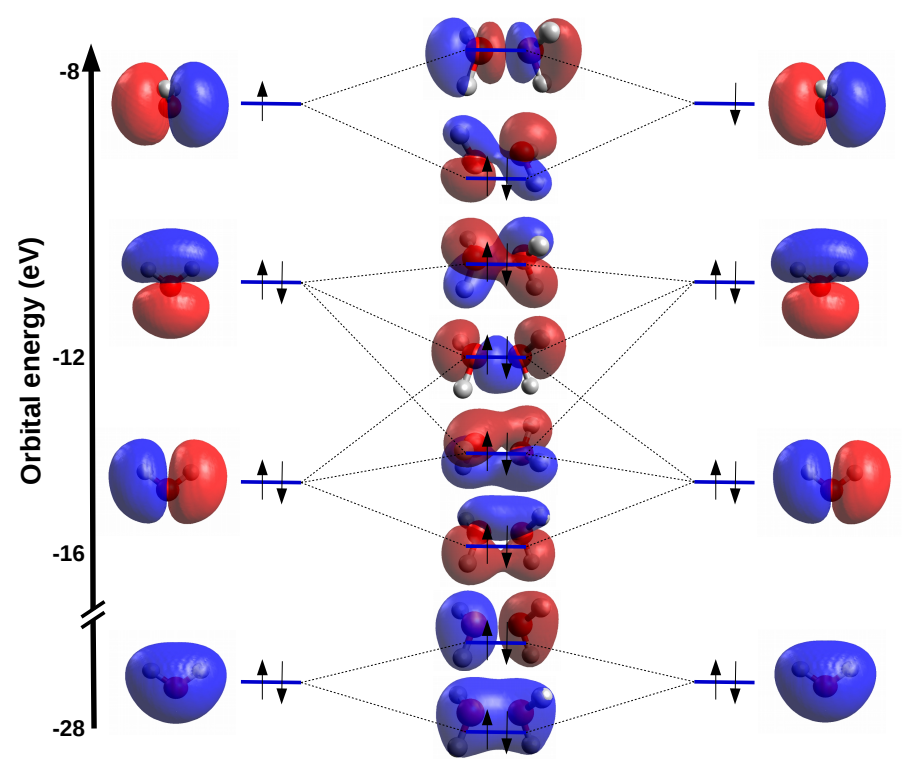

Fig. 4.8: The orbital interaction diagram of hemibonded structure for $\left(\mathrm{H}_{2} \mathrm{O}\right)_{2}^{2+}$.

$\left(\left[\mathrm{H}_{2} \mathrm{O} \cdots \mathrm{OH}_{2}\right]^{+}\right)$which is called hemibonded structures $(7,10)$. It should be noted that, for the proton transferred complex the positive charge lies mainly on the $\mathrm{H}_{3} \mathrm{O}^{+}$fragment, while for the hemibonded structure, the positive charge is delocalized between the two monomers.

Table 4.3: Optimized geometrical parameter of $\left(\mathrm{H}_{2} \mathrm{O}\right)_{2}$ and the three stationary points of $\left(\mathrm{H}_{4} \mathrm{O}_{2}\right)^{+}$. Bond lengths are in angstrom and bond angles are in degrees.

\begin{tabular}{lllll}
\hline \hline Structure & Symmetry & $r_{\mathrm{OO}}$ & $r_{\mathrm{OH}}$ & $\theta_{\text {OHO }}$ \\
\hline$\left(\mathrm{H}_{2} \mathrm{O}\right)_{2}$ & $C_{s}$ & 2.910 & 1.951 & 171.6 \\
1 & $C_{1}$ & 2.508 & 1.112 & 171.8 \\
7 & $C_{2}$ & 2.164 & 2.394 & 61.9 \\
10 & $C_{1}$ & 2.445 & 1.498 & 157.7 \\
\hline
\end{tabular}

The two minima of the water dimer radical cation (1 and 7$)$ is connected to each other with the the isomerization transition state (10). Geometries of the water dimer radical cation structures 1, 7 and 10 are shown in Fig. 4.9. The transition from 1 to 7 primarily involves the transfer of hydrogen from one $\mathrm{O}$ atom to another one.

To trace the reaction path for the two minimums of the water dimer radical cation, the calculations are performed with changes of the O-O bond length. This is shown in Fig. 4.10. The graph shows two minimums for hemibonded and proton transferred structure. Our calculation have shown that the hemibonded structure is more stable compare to the proton transferred structure. In addition, the system remain to the same structure if the OO bond length changes. The approach coordinate translates into electron interaction. 


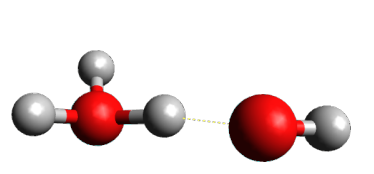

(a)

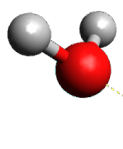

(b)

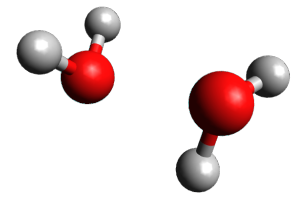

(c)

Fig. 4.9: Geometries of water dimer radical cation structures (a) proton transferred (1) (b) transition state (10) and, (c) hemibonded structure (7).

Far away (stabilizing hole), there is just repulsion, which reduces by having a hole in between. Repulsion grows again as the molecules approaches together more than the minimum structures.

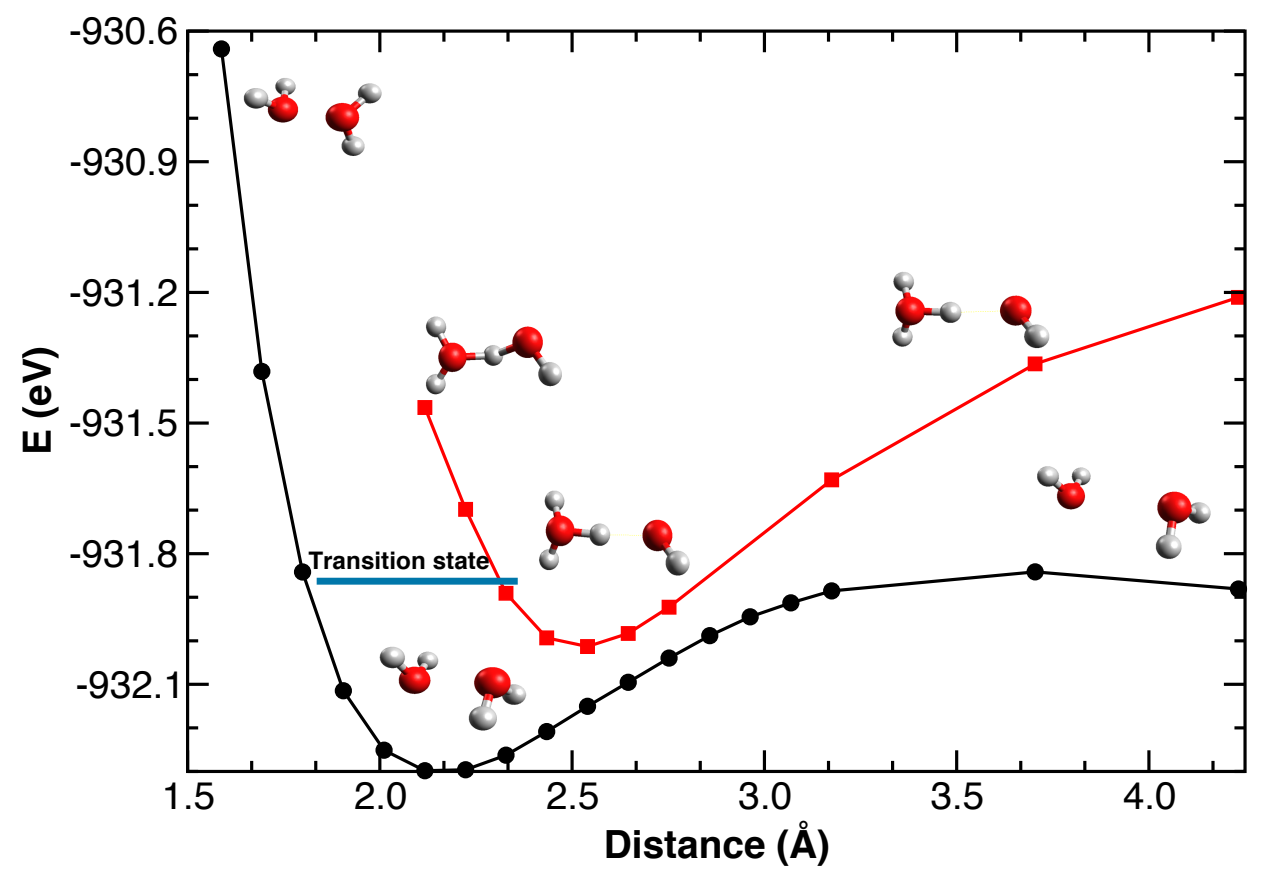

Fig. 4.10: Total energies versus $\mathrm{O}-\mathrm{O}$ bond distances for the hemibonded and the PT structure of $\left(\mathrm{H}_{4} \mathrm{O}_{2}\right)^{+}$.

According to the simulations, the two observed minima are in agreement with other calculations [139]. The interesting point is that when two molecules (PT or hemibonded structure) approach together, there is no crossing point. To get the transition state, changes of the bond angle is included into the considerations. This includes selected geometries from Tab. 4.3 which is responsible for the transition state. This is shown in Fig. 4.11. The reaction path shows that the hydrogen donor water rotates its hydrogen atom toward the 
hydrogen acceptor water. This connects to the transition state. Then the rotated hydrogen moves toward the oxygen of the hydrogen acceptor water. An energy barrier of $0.43 \mathrm{eV}$ is calculated for the PT transfer process involving hemibonded structure.

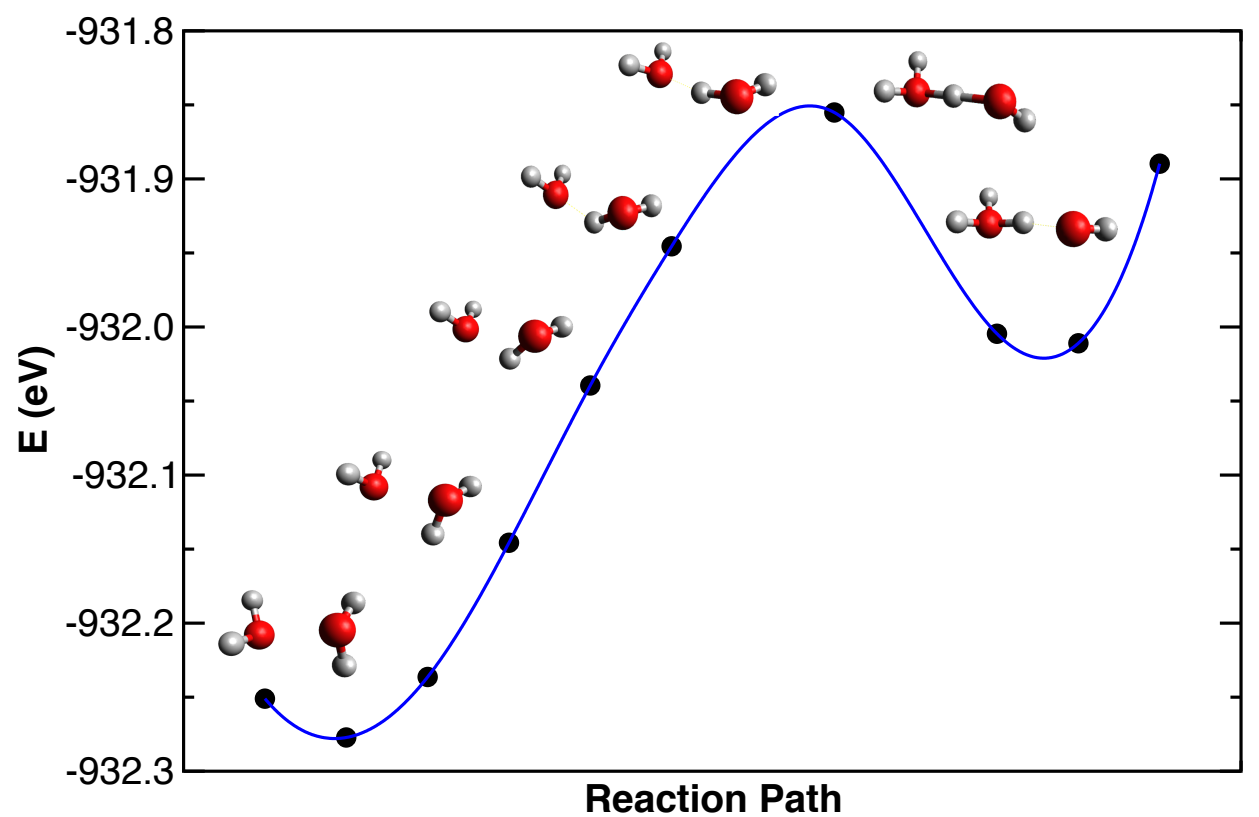

Fig. 4.11: Energy profile along the reaction path coordinate for the proton transfer process involving water dimer radical cation structures.

\subsubsection{Potential energy surfaces of $\left(\mathrm{H}_{2} \mathrm{O}\right)_{2}^{+}$}

The potential energy surface (PES) is a relationship - mathematical or graphical - between the energy of a molecule and its geometry. Many important concepts such as global minimum or local minimum and their reaction path can be grasped with the PES. For the water dimer radical cation, the PES is shown in Fig. 4.12a. In this case the three internal coordinates, the $\mathrm{O}-\mathrm{O}$ distance, $\mathrm{O}-\mathrm{H}$ distances and $\mathrm{O}-\mathrm{H}-\mathrm{O}$ angle determine the geometrical changes. The relaxation to the equilibrium structure involves mostly to $\mathrm{O}-\mathrm{O}$ and $\mathrm{OH}$ distances. The distance of the share proton from the oxygen atom increases from $0.96 \AA$ to $1.46 \AA$. The $\mathrm{O}-\mathrm{O}$ distance decreases from $2.9 \AA$ to $2.0 \AA$. The three dimensional PES which is shown, is the energy relative to the $\mathrm{O}-\mathrm{O}$ and $\mathrm{O}-\mathrm{H}$ distances.

Mathematically, a stationary point is one at which the first derivative of the potential energy with respect to each geometric parameter is zero. At both the transition state and the minimum $\partial E / \partial R=0$ for all geometric coordinates $\mathrm{R}$. At the transition state $\partial^{2} E / \partial R^{2}<0$ for $\mathrm{R}$ is equal to the reaction coordinate and $>0$ for all other $\mathrm{R}$ (along all 


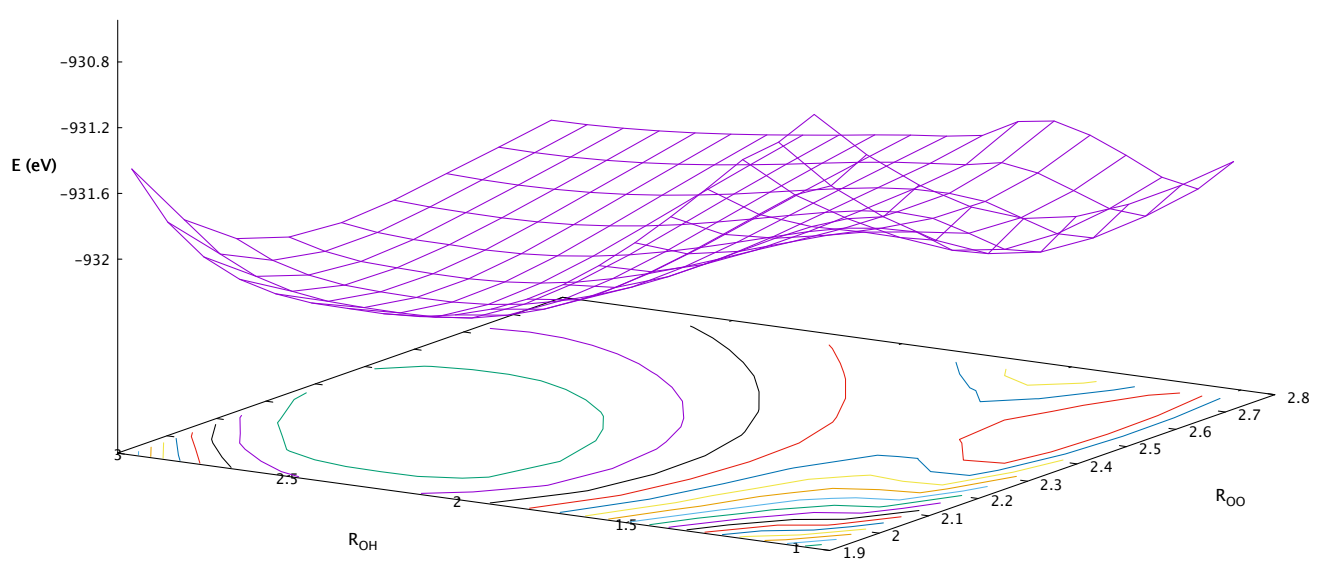

(a)

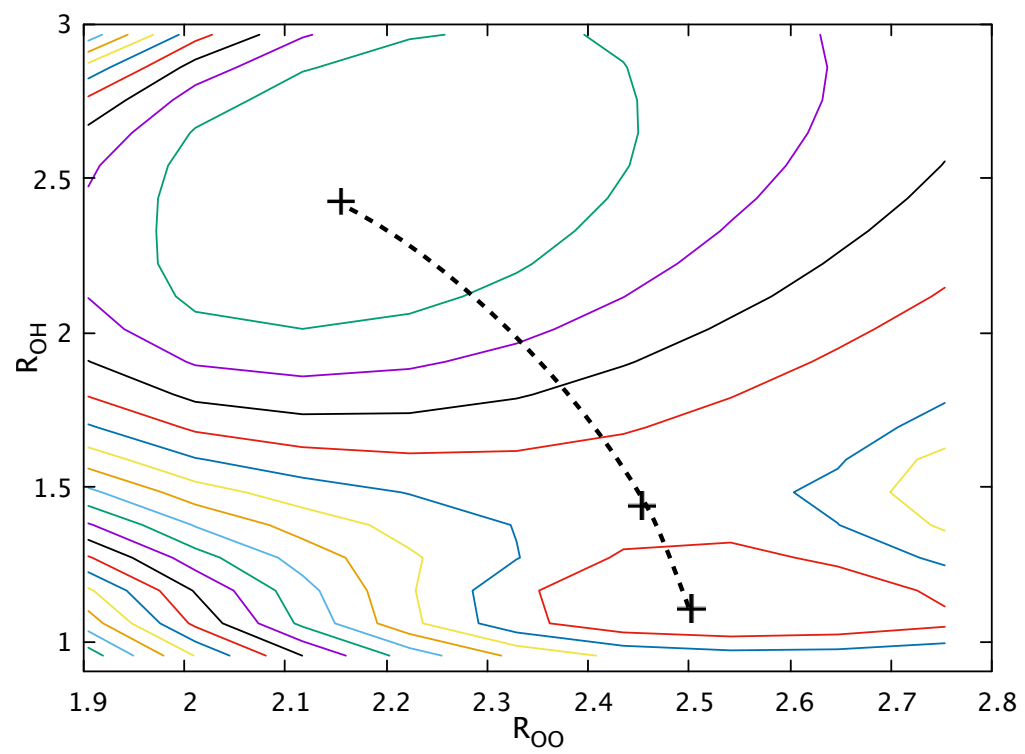

(b)

Fig. 4.12: (a) The 3D and also 2D slice of the PES for water dimer radical cation structure. (b) The lowest energy pathway linking the two minima. Transition state starting from a point in the reactant valley and another in the product valley attracted to each other and move toward a transition state in a PES for water dimer radical cation structure.

other directions). At a minimum $\partial^{2} E / \partial R^{2}>0$ for all $\mathrm{R}$ (along all directions). 
Inspection of Fig. 4.12a shows that the transition state linking the two minima represents a maximum along the direction of the pathway, while along all other directions it is a minimum. This is a characteristic of a saddle-shaped surface, and the transition state is called a saddle point. The saddle point lies at the "center" of the saddle-shaped region and is, like a minimum, a stationary point. Mathematically, minima and saddle points differ in that although both are stationary points (they have zero first derivatives). A minimum is a minimum in all directions, but a saddle point is a maximum along the reaction coordinate and a minimum in all other directions. It should be noted that minimum and maximum can be distinguished by their second derivatives.

The 2D PES scanning along O-H and O-O distances is shown in Fig. 4.12b. The dashed line represents possible pathway on the potential energy surface for linking the two minima. This kind of calculations helped us single out the leading reaction coordinates during the ionization process. In the case of ionization into the PT state, we observed a very fast proton transfer process. This indicates a direct barrierless process. The most probable mechanism would be an elongation of the $\mathrm{O}-\mathrm{H}$ bond until the hydrogen gets trapped by the other oxygen atom. Such a direct process without any adjustment of the oxygen atom positions is, however, accompanied by a small, yet noticeable barrier $(0.35 \mathrm{eV})$. Our calculations suggest that the process starts with a change in the $\mathrm{O}-\mathrm{O}$ distance. The twodimensional scan confirms the suggested mechanism. First the O-O distance gets smaller, and only once a heavy atom cage is conveniently arranged the proton is transferred.

\subsection{Surface calculations}

So far we have only done spin-polarized calculations for molecules to get more insight into the reaction mechanism. In the following we try to apply this mechanism on the surface. In order to study a surface, our ideal model would be a slice of material that is finite along the normal vector of surface, but infinite in two other directions. In this model, the atoms in the $x$ and $y$ directions fill the entire supercell, but in the top portion of the supercell empty space has been applied. This empty space which separates periodic images of the slab along the $z$ direction is so-called the vacuum space. This model defines as a slab model. It should be noted that the vacuum space must be large enough to make the interaction between the top of one slab on the bottom of the next. Thus, the supercell really defines two surfaces, an upper and lower surface.

To get the important characteristics of a real surface, atleast five layers of the material are necessary. Typically, more that five layers are better, but using more layers means using more computational time. Furthermore, number of layers depend on the nature of the material studied and the property of interest. For our purpose, water oxidation, this amount of layers are enough to compromise between computational cost and physical accuracy.

To understand how DFT calculations can be used to examine surface reactions, (001) surface of $\mathrm{CaMnO}_{3}$ and aqueous interfaces have been chosen. Our slab contains five layers 
of $\mathrm{CaMnO}_{3}$ that is three $\mathrm{MnO}_{2}$ layers and two $\mathrm{CaO}$ layers. In the top and bottom layer, $\mathrm{MnO}_{2}$ layer is placed. Many chemical reactions on surface can be happened involving the adsorption of individual $\mathrm{H}$ atoms. So, knowing the characteristics of these H-atoms on $\mathrm{CaMnO}_{3}$ (001) surface would be a necessary step toward describing any of reactions. If we look at the (001) surface, and try to find out several possibilities for water decomposition on surface, the most favorable structure is to have a $\mathrm{OH}$ group on one $\mathrm{Mn}$-site, $\mathrm{OH}_{2}$ to the nearest neighbor Mn-site. The cleaved proton migrates to an adjacent oxygen ion on the surface. This is illustrated in Fig. 4.13.

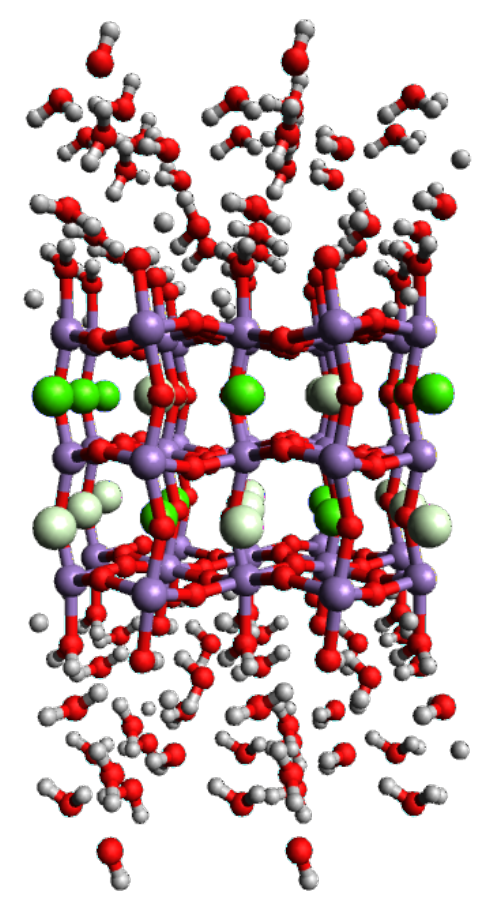

Fig. 4.13: Illustration of binding sites of $\mathrm{H}_{2} \mathrm{O}$ on $\mathrm{CaMnO}_{3}(001)$. Coordinates used for this illustration come from a fully relaxed $\mathrm{PBE} 0 \mathrm{r}$ calculation. The red spheres represent the $\mathrm{O}$ ions and the gray spheres show the $\mathrm{H}$ atoms. The Green (white) spheres represent $\mathrm{Ca}(\mathrm{Pr})$ ions and the purple spheres show Mn cations. Dashed lines stand for the hydrogen bond.

Using water layers above and below the slab prevents the occurrence of a large dipole moment, which would distort the results. The water layers are designed in such a way that the oxygen atoms sit on the $\mathrm{Mn}$ atomic positions of the $\mathrm{MnO}_{2}$ layer. The orientation of the protons occurs in such a way that the most realistic hydrogen bonding network is possible, in which each oxygen ion receives two hydrogen bonds, and each hydrogen atom forms a bridge. The final relaxation of the slab is carried out by PBE0r functional.

\subsubsection{Computational details}

All calculations were performed using the PBE0r local hybrid functional. The plane wave cutoff for the auxiliary wave function is $40 \mathrm{Ry}$ and $80 \mathrm{Ry}$ for the density. The $\mathrm{CaMnO}_{3}$ 
(001) surface was modeled as a periodically repeated slab of three $\mathrm{MnO}_{2}$ layers and two $\mathrm{CaO}$ layers, with a rectangular surface cell of dimensions $5.27 \times 5.27 \AA^{2}$, corresponding to $6 \mathrm{CaMnO}_{3}$ formula units. A k-point grid of $4 \times 4 \times 1$ points is chosen. This means that along the first and second reciprocal lattice vector there are four k-points and along the third reciprocal lattice vector one k-point. There are $16 \mathrm{k}$-points in total, however due to the symmetry, the number of $\mathrm{k}$ points is reduced to 10 . It should be noted that, If the vacuum region is large enough, the electron density tails off to zero at a short distance from the edge of the slab. This means that accurate results are possible using just one $\mathrm{k}$ point in the normal surface direction. To simulate the $\mathrm{CaMnO}_{3} /$ water interface, fully solvated slab in liquid water (shown in Fig. 4.13) were utilized. The large $z$-component of the third lattice vector ensures that the distance between two water molecules from different slaps is greater than $12 \AA$, so that the system can be understood as a surface.

To keep the symmetry and get rid of the dipole interaction, on the top and bottom of the slab, we have equivalent surfaces. Both surfaces are covered by 8 water-molecules. To investigate the kinetics of proton/electron transfer, we sequentially add holes with integer charge into the system. First we tried to localize the hole between the O-O bond by changing the distance then, to avoid the errors associated with charged systems under periodic boundary conditions, we neutralize the charge associated with a hole by removing a proton from an adsorbed $\mathrm{H}_{2} \mathrm{O}$ molecule. This results in an adsorbed $\mathrm{OH}^{-}$on the surface of the slab.

The optimized $x y z$ coordinates of the calculated adsorbates on $\mathrm{CaMnO}_{3}(001)$ are given in Appendix. B.

\subsubsection{Surface relaxation and reconstruction}

We placed atoms in our slab model in order to create a five layers slab. The position of the atoms were the ideal, bulk positions of material. Since the coordination of atoms in the surface is reduced compared with those in the bulk, it is natural to expect that the spacings between layers near the surface might be somewhat different from those in the bulk. this phenomena is called surface relaxation.

It is important to recognize that DFT calculations may not yield accurate information about a surface merely by allowing surface to relax. Most of the surfaces undergo reconstructions in which surface atoms form new bonds. A very clear example of this phenomena is given by the $\mathrm{Si}(100)$ surface. In our case for $\mathrm{CaMnO}_{3},(001)$ plane doesn't have any kind of reconstruction while (110) surface has.

\subsection{Oxygen evolution reaction}

One of the most widely used mechanisms for the OER [142, 143] is based on the following four sequential proton-electron transfer (PET) steps: 


$$
\begin{aligned}
& { }^{*} \mathrm{OH}_{2}+h^{+} \rightarrow{ }^{*} \mathrm{OH}+\mathrm{H}^{+} \\
& { }^{*} \mathrm{OH}+h^{+} \rightarrow{ }^{*} \mathrm{O}+\mathrm{H}^{+} \\
& \mathrm{H}_{2} \mathrm{O}+{ }^{*} \mathrm{O}+h^{+} \rightarrow{ }^{*} \mathrm{OOH}+\mathrm{H}^{+} \\
& { }^{*} \mathrm{OOH}+h^{+} \rightarrow \mathrm{O}_{2}+{ }^{*}+\mathrm{H}^{+}
\end{aligned}
$$

where ${ }^{*} X$ denotes an adsorbed $X$ species, $\mathrm{H}_{2} \mathrm{O}$ is a water molecule, and $\mathrm{H}^{+}$is a solvated proton. In this scenario, $\mathrm{O}_{2}$ evolves from an adsorbed ${ }^{*} \mathrm{OOH}$ species which is created from the association of two deprotonated water molecules. In This work, the mechanism is based on a hydroxide species $\left({ }^{*} \mathrm{OH}\right)$ which is obtained from adsorption of water on the $\mathrm{MnO}_{2}$ surface. We will consider the following reaction mechanism:

$$
\begin{aligned}
& { }^{*} \mathrm{OH}+h^{+}+\mathrm{H}_{2} \mathrm{O}(l) \rightarrow{ }^{*} \mathrm{HOOH}^{-}+\mathrm{H}^{+} \\
& { }^{*} \mathrm{H}_{2} \mathrm{O}_{2}^{-}+h^{+} \rightarrow{ }^{*}--\mathrm{O}_{2} \mathrm{H}_{2} \\
& { }^{*}--\mathrm{O}_{2} \mathrm{H}_{2}+h^{+} \rightarrow{ }^{*} \mathrm{OOH}+\mathrm{H}^{+} \\
& { }^{*} \mathrm{OOH}+h^{+} \rightarrow{ }^{*} \mathrm{O}_{2}^{-}+\mathrm{H}^{+} \\
& { }^{*} \mathrm{O}_{2}^{-} \rightarrow{ }^{*}--\mathrm{O}_{2}
\end{aligned}
$$

where -- denotes a physical adsorption. In order to ensure the charge neutrality, the charge of the metal cation also changes during the reaction steps. We studied this mechanism in more detail at the aqueous $\mathrm{CaMnO}_{3}$ (001) interface.

It should be noted that, no universal consensus on a detailed mechanism of oxygen evolution reaction on $\mathrm{CaMnO}_{3}$ has yet emerged [144, 145, 146, 147, 148]. An important question is whether the atomic pathways are the same or different on $\mathrm{CaMnO}_{3}$ compared to other perovskite. And, how do the structural differences between $\mathrm{CaMnO}_{3}$ and $\mathrm{PrMnO}_{3}$ affect the OER pathways? Recent studies [144, 146] have shown that $\mathrm{PrMnO}_{3}$ is generally a better catalyst than $\mathrm{CaMnO}_{3}$, which has been attributed to reasons such as band gap, charge-carrier lifetime, polaronic excitation, and higher charge-carrier mobility. There is evidence that $\mathrm{PrMnO}_{3}$ has a higher activity and stability against corrosion than $\mathrm{CaMnO}_{3}$. The mechanism of catalytic water oxidation on the manganite surfaces has been the subject of several recent studies. To obtain more insights, in this work we focus on $\mathrm{CaMnO}_{3}(001)$ to determine the complete pathway and kinetics of oxygen evolution using local hybrid DFT-based electronic structure calculations.

\subsubsection{The initial situation: water-covered $\mathrm{CaMnO}_{3}$ surface}

The calculated DoS of aqueous $\mathrm{CaMnO}_{3}$ (001) interface is shown in Fig. 4.14. The filled states below the Fermi level are O-p states from $-7 \mathrm{eV}$ to $0.5 \mathrm{eV}$, and the empty states above the Fermi level are Mn- $d$ orbitals from $2 \mathrm{eV}$ to $7 \mathrm{eV}$. There is no contribution of 
water molecules near the Fermi level. The band gap is about $1.43 \mathrm{eV}$, has close to the calculated band gap of bulk $\mathrm{CaMnO}_{3}$. The Ca contributions are far from Fermi level and overlaps with neither $\mathrm{O}-2 p$ nor Mn-3d states. The filled $t_{2 g}$ states in the O-band can be divided into bonding states, in the lower half of the valence band and antibonding states in the upper half. The bonding states of $t_{2 g}$ character are accompanied by the bonding states of $e_{g}$ character. The conduction band is formed by the overlapping contributions of the majority-spin $e_{g}$ states in the lower part and the minority-spin $t_{2 g}$ states. The conduction band is separated from a nearly isolated band of minority spin $e_{g}$ states at high energies. The oxygen atoms are all spin non-polarized ( $\mathrm{O}$ magnetic moment is zero) because in density of states, O- $2 p$ is totally symmetric for all oxygen atoms.

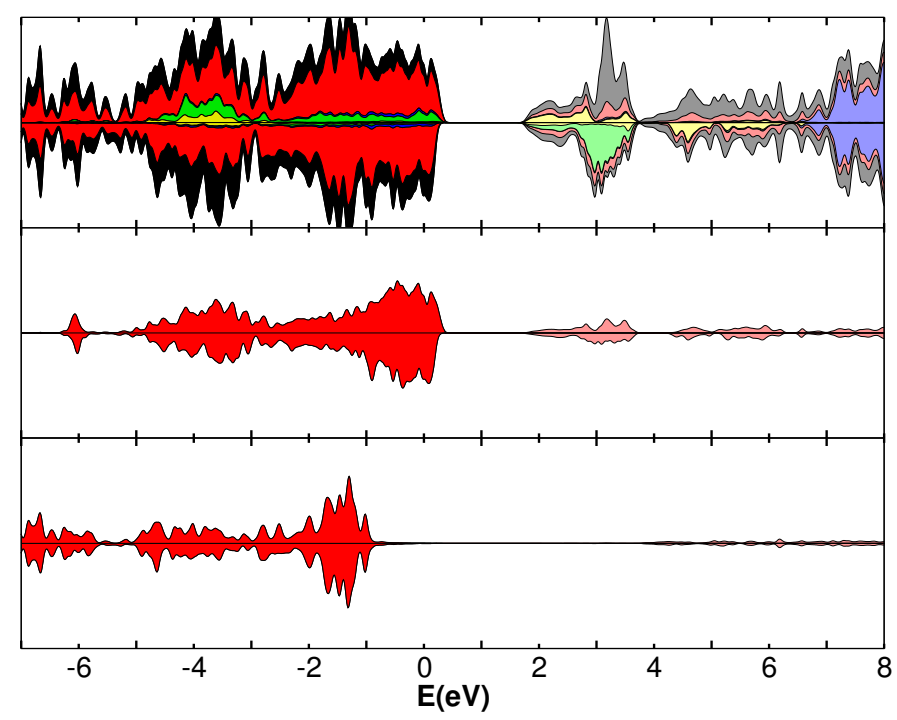

Fig. 4.14: DoS of the non-ionized initial structure. The three graphs indicate different DoS. The top figure shows the total DoS (black envelope) with the projected DoS for O- $p$ (red), Mn- $t_{2 g}$ (green), Mn- $e_{g}$ (yellow), and Ca- $d$ (blue). Projected DoS are stacked on top of each other. The two spin densities are shown with opposite sign. For the Mn ion, the projected DoS is consider with one majority spin direction. The graph in the middle shows the oxygen contribution in the bulk $\mathrm{CaMnO}_{3}$. The bottom graph shows the oxygen contribution in the water molecules.

Based on the electronic structure, the most likely scenarios for oxygen evolution reactions are thus bond formation between the hydroxide group and water molecule, the hydroxide group and surface O-atom, or the water molecule and surface O-atom. Of these possibilities, the bond formations between the hydroxide group and water molecule and also between the hydroxide group and surface O-atom were calculated in great details to understand the reaction pathway.

It should be noted that the light generates hole which migrates in to bonds. The orbitals of states just below the valence band edge (HOMO) have their largest contributions from free e-h pairs of surface O-atoms. So, OH group which is attached to the surface and 
Surface O-atoms are of our interest in this study.

The main focus is on the three oxygen atoms in vicinity of the surface (Fig. 4.15). O2 is present as a $\mathrm{MnOH}$ species. O3 belongs to the middle water molecule. These two oxygen atoms are connected to each other with a bridging hydrogen and a hydrogen bond. The hole can breaks down the hydrogen bond and rotates $\mathrm{O} 3$ to form a hemibonded structure between $\mathrm{O} 2$ and $\mathrm{O} 3$. Then, the overlap of the $p_{z}$ orbitals at the oxygen atoms arises. The bond lengths of the Mn-O2 and O2-O3 are 1.957 $\AA$ and $2.657 \AA$, respectively.

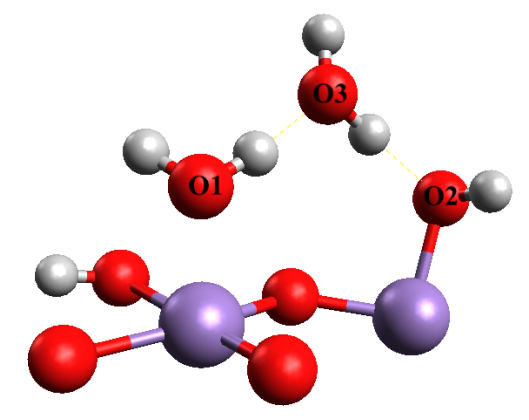

Fig. 4.15: Illustration of the important surface oxygen atoms. Water interface and two different species of ${ }^{*} \mathrm{OH}$ and ${ }^{*} \mathrm{OH}_{2}$. Labels are shown the oxygen atoms which participate to make O-O bond. O2 and O3 come close to form a hemibonded structure.

\subsubsection{First Proton-Electron Transfer}

\section{Ionized system}

In the ionized system, the created hole shows a tendency to localize at the bulk. However, since a radical $\mathrm{OH}$ is formed on the surface, the hole migrates through the bulk to the solid-water interface. It should be noted that the two holes (one for each side) are inserted into the system. In the case of hole localization at $\mathrm{O} 3$, a rotation of the molecule must take place to make hemibonding interaction.

The effect of the hole doping has been investigated by performing simulations on the initial structure and removing one electron per surface. Basically, nothing has changed in the structure. $\mathrm{O} 2$ and $\mathrm{O} 3$ have the same orientation to each other as in the unoxidized system. The O2-O3 bond lengths is $2.726 \AA$. The change in the O2-O3 is about $2.5 \%$. The rotation of the water molecule is not visible. The hydrogen bonding network has not changed significantly due to ionization.

The calculated DoS for the ionized system is shown in Fig. 4.16a. The states near the HOMO are now no longer dominated by the oxygen $p$ orbitals from the bulk oxide layers, but the water layer also has contribution in the highest occupied orbitals. In addition, unoccupied states lie directly at the valence band edge. The highest occupied states as well as the lowest unoccupied states sit in the inner oxide layers as well as the outer water layer. The generated holes thus partially migrate to the top water molecules. Within the 
hydrogen bridge network, however, no bond formations or relevant geometry changes can be recognized.

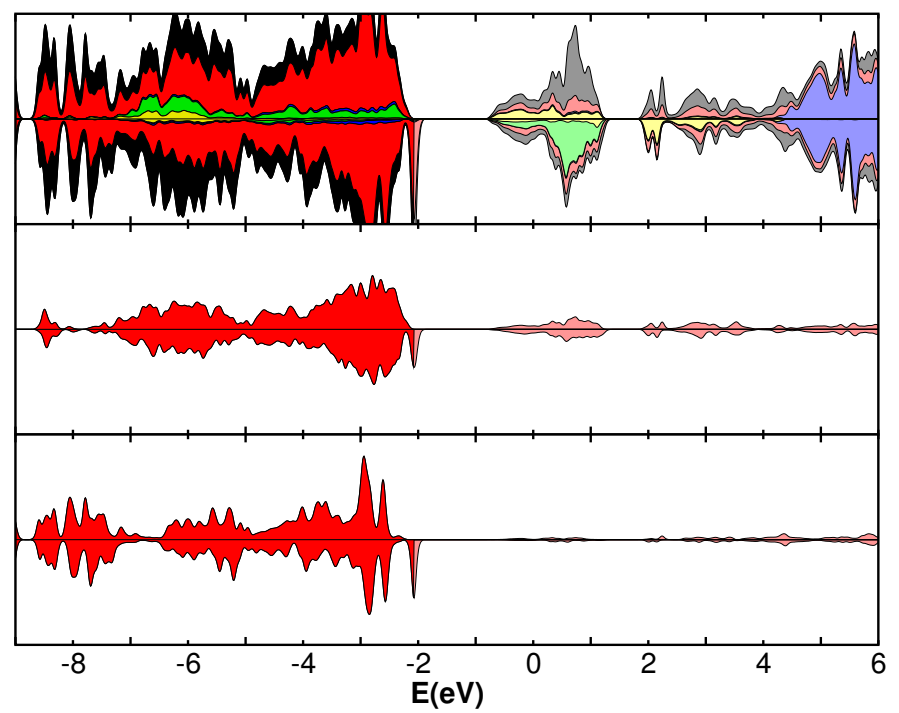

(a)

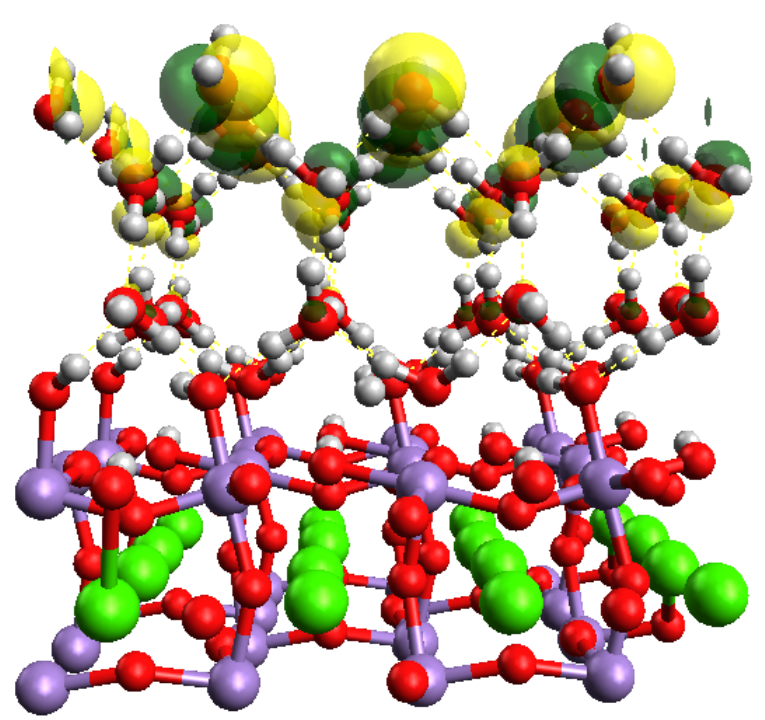

(b)

Fig. 4.16: (a) DoS of the ionized initial structure. The three graphs indicate different DoS. The top figure shows the total DoS (black envelope) with the projected DoS for O- $p$ (red), Mn- $t_{2 g}$ (green), Mn- $e_{g}$ (yellow), and Ca- $d$ (blue). Projected DoS are stacked on top of each other. The two spin densities are shown with opposite sign. For the Mn ion, the projected DoS is consider with one majority spin direction. The graph in the middle shows the oxygen contribution in the bulk $\mathrm{CaMnO}_{3}$. The bottom graph shows the oxygen contribution in the water molecules. (b) Orbital representation of the state in the valence band top which is created by hole doping. 
The molecular wave function of the hole state is shown in Fig. 4.16b. The generated hole is localized on the top layer of water. While the bulk orbitals are strongly stabilized, the water molecules are influenced energetically by hole doping. Water molecule contributions come close to the Fermi level and they are involved in the HOMOs / LUMOs.

\section{$\mathrm{OH}$ radical interaction with water}

In the first proton-electron transfer, Eq. 4.2, a surface-trapped hole $\left(h^{+}\right)$reacts with an adsorbed $\mathrm{H}_{2} \mathrm{O}$ molecule to release a proton and creates a ${ }^{*} \mathrm{OH}$ radical. But on $\mathrm{CaMnO}_{3}$ (001), water adsorbs at the 5-fold Mn surface site (see Fig. 4.13) and we have two ${ }^{*} \mathrm{OH}$ radical and ${ }^{*} \mathrm{OH}_{2}$ species on surface. So, the localization site for a hole would be a different story. The hole breaks down a hydrogen bond between ${ }^{*} \mathrm{OH}$ radical and $\mathrm{H}_{2} \mathrm{O}$ molecule to create a O-O bond like what we discussed earlier for dimeric water. Then the hole localized between the two oxygen as shown in Fig. 4.17.

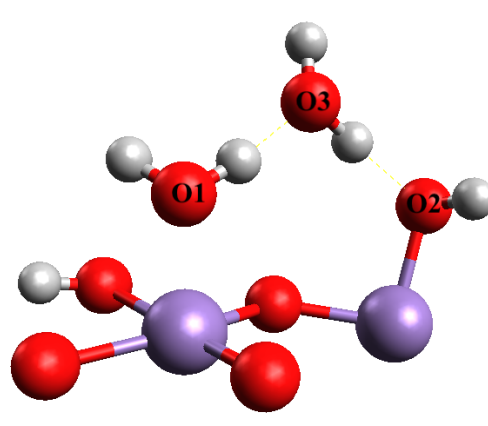

(a)

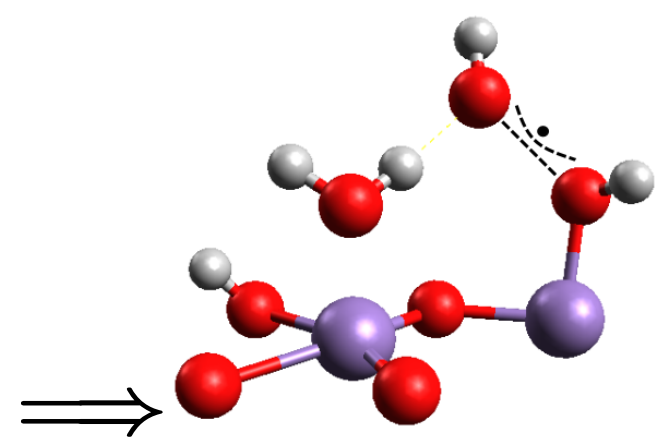

(b)

Fig. 4.17: Molecular representation of the unit cell with labels for the important surface atoms. (a) Water interface and two different species of ${ }^{*} \mathrm{OH}$ and ${ }^{*} \mathrm{OH}_{2}$, and (b) breaking down a hydrogen bond and removing proton from water molecule and making O-O bond.

One of 2-fold coordinated bridging oxygen $\left(\mathrm{O}_{b r}\right)$ makes a bond with $\mathrm{H}$. Thus, surface is covered partially by $\mathrm{H}$ atoms. To identify the most favorable site(s), we carried out several separate simulations. These calculations showed that a hole located between the ${ }^{*} \mathrm{OH}$ and $\mathrm{H}_{2} \mathrm{O}$ remains stable during a simulations and after relaxation a $\mathrm{H}^{+}$releases from $\mathrm{H}_{2} \mathrm{O}$ and transfers to the top layer of water to make $\mathrm{H}_{3} \mathrm{O}^{+}$. We thus focused on a hole initially localized at $\mathrm{O}-\mathrm{O}$ bond. In all cases, we obtained a barrier for PT of $0.5 \mathrm{eV}$ and found the hole to remain localized on the water during the PT. This activation energy is similar to the dimeric water studies. For comparison, we also examined the general mechanism proposed by Norskov et al. for $\mathrm{CaMnO}_{3}$ using one of the configurations with the hole at ${ }^{*} \mathrm{OH}_{2}$. We found this mechanism to be energetically unfavorable on $\mathrm{CaMnO}_{3}(001)$ and to have a barrier of $0.8 \mathrm{eV}$. This is higher than the barrier $(0.5 \mathrm{eV})$ of the PET step 4.6.

Electronically, states below the Fermi level are kept the same, but states above the Fermi level are changed. Mn- $d$ orbitals as shown in Fig. 4.18 have the same contributions 
but there is a contribution from water molecules near the Fermi level. The water O- $p$ state near the Fermi level, is an antibonding orbital of two oxygens which come close together. So, it makes the band gap smaller and about $1.0 \mathrm{eV}$. The Ca contributions are far from Fermi level without any changes. Thus, based on the electronic structure, the bond formation between the hydroxide group and water molecule is applicable. This bond formations thermodynamically is more favorable than having a bare oxygen on surface however, it creates a peroxide which has influences on the stability of catalyst.

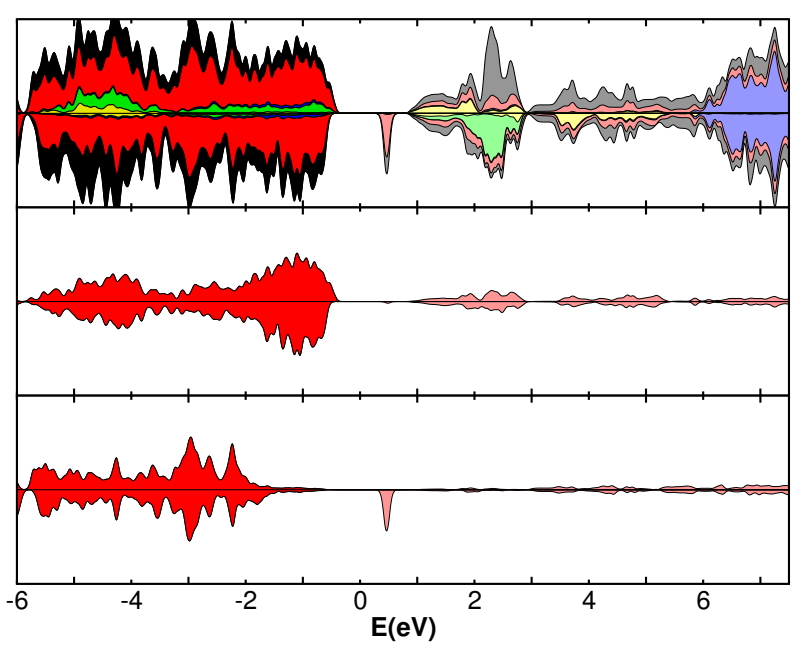

(a)

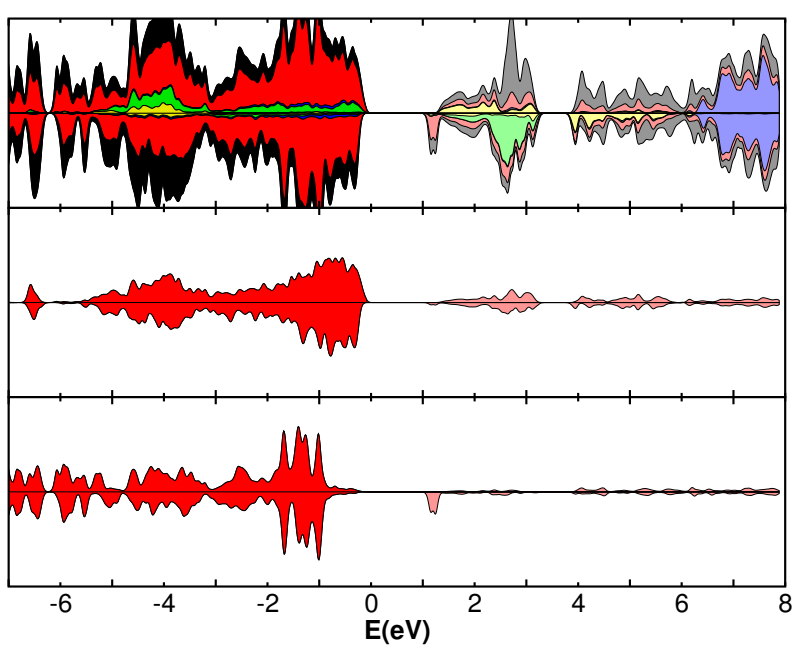

(b)

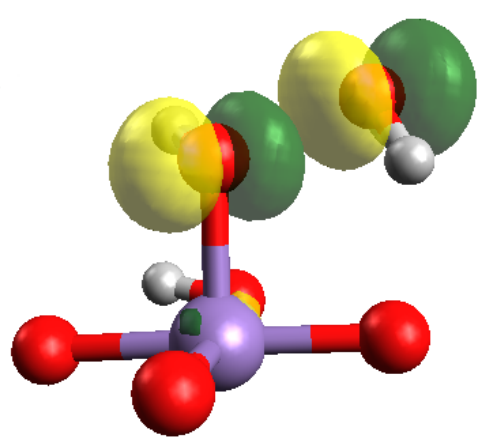

(c)

Fig. 4.18: DoS of the (a) ionized initial structure and (b) deporotonated structure. The three graphs indicate different DoS. The top figure shows the total DoS (black envelope) with the projected DoS for O-p (red), Mn- $t_{2 g}$ (green), Mn- $e_{g}$ (yellow), and Ca-d (blue). Projected DoS are stacked on top of each other. The two spin densities are shown with opposite sign. For the Mn ion, the projected DoS is consider with one majority spin direction. The graph in the middle shows the oxygen contribution in the bulk $\mathrm{CaMnO}_{3}$. The bottom graph shows the oxygen contribution in the water molecules. (c) Orbital representation of the state in the bottom part of the conduction band which is created by hole doping. 


\section{Deprotonated water molecule}

The observation of a primary ionized effect suggests that the loss of a hydrogen bridge bond from the $\mathrm{OH}$ interaction with the water molecule involves proton migration. The proton at $\mathrm{O} 3$ migrates to the overlying water molecule and then points towards O3. The proton makes $\mathrm{H}_{3} \mathrm{O}^{+}$ion on the upper layer of water. It was therefore obvious to remove exactly this proton. Without constraints, this state is stable and proton migrates to the top water layer. The O2-O3 bond length is $1.98 \AA$.

The DoS for deporotonated water molecule is shown in Fig. 4.18b. Mn- $d$ orbitals and O- $p$ orbitals of $\mathrm{CaMnO}_{3}$ have the same contributions but there is a different contribution from the water molecules near the Fermi level. The state in the band gap moves toward the conduction band and makes band gap larger. The band gap is $1.2 \mathrm{eV}$. This state is an antibonding orbital of two oxygens which come close together. The main effect of deporotonated structure for the O- $p$ states of the water molecules, is a upward shift of occupied orbitals. These states come close to the Fermi level.

\section{$\mathrm{OH}$ radical interaction with Surface O-atom}

Recently, experiments $[149,131]$ have shown that OER on highly active perovskites such as $\mathrm{La}_{1-x} \mathrm{Sr}_{x} \mathrm{CoO}_{3-\delta}$ contains the lattice oxygen $\mathrm{O}_{\text {surf }}$. In addition, computational studies using DFT showed that the conventional adsorbate evolution mechanism is more favorable [129]. For $\mathrm{CaMnO}_{3}$, it was found that by introducing constraints, the hole can not be localized between the $\mathrm{O} 2$ and $\mathrm{O}_{\text {surf }}$. However, no statements have yet been made about the stability of these states. In other words, removing the constraints moves atoms away from each other. Therefore, this pathway is not likely.

\subsubsection{Second Proton-Electron Transfer}

For the second PET, the following reaction mechanism is suggested:

$$
{ }^{*} \mathrm{H}_{2} \mathrm{O}_{2}^{-}+h^{+} \rightarrow{ }^{*}--\mathrm{O}_{2} \mathrm{H}_{2}
$$

Another photoexcited hole is needed to initiate the second PET. This hole migrates in proximity of ${ }^{*} \mathrm{OH}$. The second hole localizes between $\mathrm{O} 2$ and $\mathrm{O} 3$ and make a new hydrogen peroxide compound. The O2-O3 bond length is $1.46 \AA$. The oxygen atoms in the hydrogen peroxide ion have an oxidation state of -1 . The Mn-O2 distance was $1.96 \AA$ in the initial structure and became $1.95 \AA$ in the first PET. Then it grows to $2.5 \AA$ in this reaction step. It seems to be a physically adsorbed hydrogen peroxide. A possible reason for the transition from chemisorption to physisorption is the further ionization. Without cleavage of the Mn-O bond, O2 would not have the required electrons for this reaction.

The calculated DoS of the second hole doping is shown in Fig. 4.19a. The filled valence band for $\mathrm{CaMnO}_{3}$ is quite the same. While the the filled valence band for the water 
molecules form a contribution near the Fermi level and the main contribution are shifted downward. In the conduction band, $\mathrm{CaMnO}_{3}$ has a same contributions, but for the water molecules the $\sigma$ bond between the $\mathrm{O} 2$ and $\mathrm{O} 3$ is shifted upward with two spin directions.

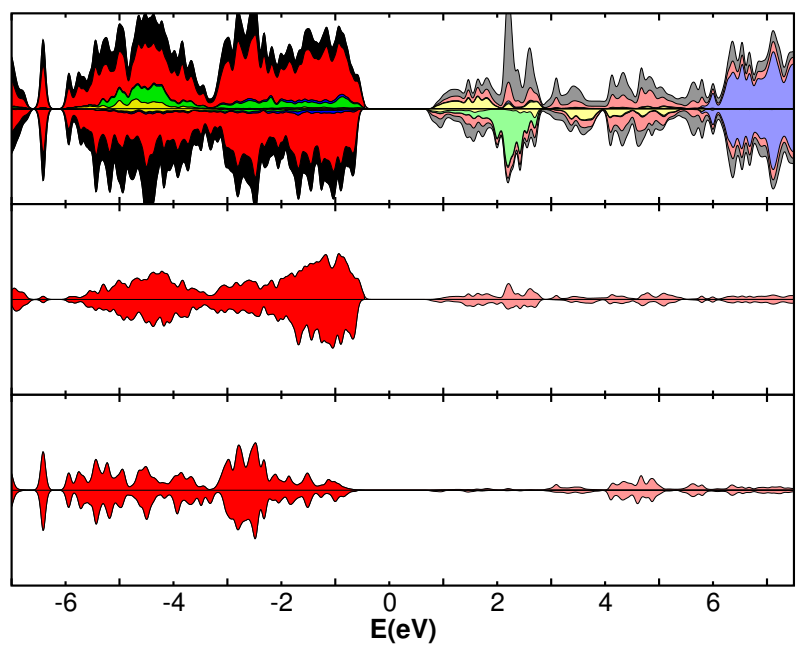

(a)

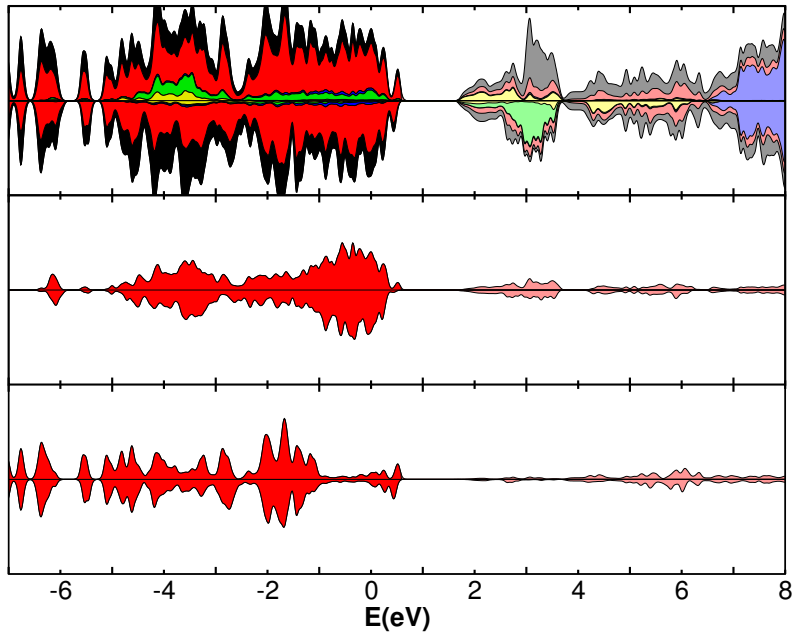

(b)

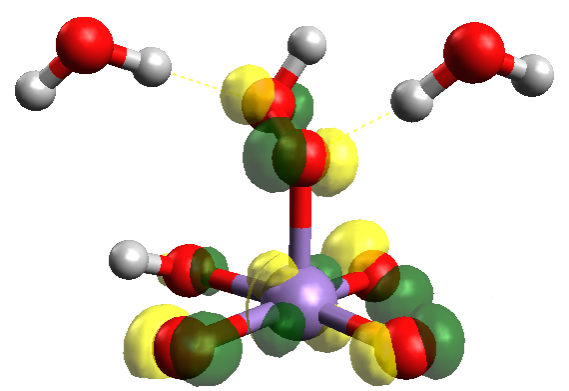

(c)

Fig. 4.19: DoS of the (a) second hole doped structure and (b) second deprotonated structure. The three graphs indicate different DoS. The top figure shows the total DoS (black envelope) with the projected DoS for O- $p$ (red), Mn- $t_{2 g}$ (green), Mn- $e_{g}$ (yellow), and Ca- $d$ (blue). Projected DoS are stacked on top of each other. The two spin densities are shown with opposite sign. For the Mn ion, the projected DoS is consider with one majority spin direction. The graph in the middle shows the oxygen contribution in the bulk $\mathrm{CaMnO}_{3}$. The bottom graph shows the oxygen contribution in the water molecules. (c) Orbital representation of the valence band top states in the second deprotonated structure.

\section{Deprotonated hydrogen peroxide}

The next step of the mechanism is to deprotonate the previous hydrogen peroxide species. In principle, two sites are available for deprotonation. The proton from $\mathrm{O} 2$ or the proton 
from $\mathrm{O} 3$ and make a hydronium ion on the overlying water molecule. Both variants are calculated. Comparing the energies shows that the proton transfer from $\mathrm{O} 2$ is more stable by $0.3 \mathrm{eV}$. Structurally, Mn-O2 bond length becomes smaller for both reactions by $0.5 \AA$.

The calculated DoS is given in Fig. 4.19b.

The $\sigma$ bond between $\mathrm{O} 2$ and $\mathrm{O} 3$ is located in the higher energy range of the conduction band. The $\pi$ bond between $\mathrm{O} 2$ and $\mathrm{O} 3$ is located in the higher occupied orbitals. The $\pi$ orbital is in the HOMO and next hole will place in this orbital. The optimized geometry from this pathway is shown in Fig. 4.20. Our calculations revealed that the $\mathrm{OH}$ radical at the surface turns to the hydrogen peroxide $\mathrm{H}_{2} \mathrm{O}_{2}$ and then formation of the ${ }^{*} \mathrm{OOH}$ species.

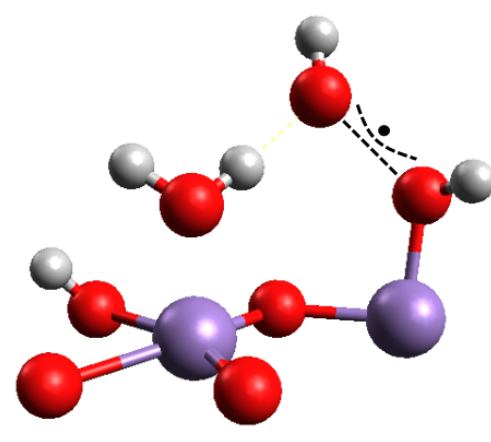

(a)

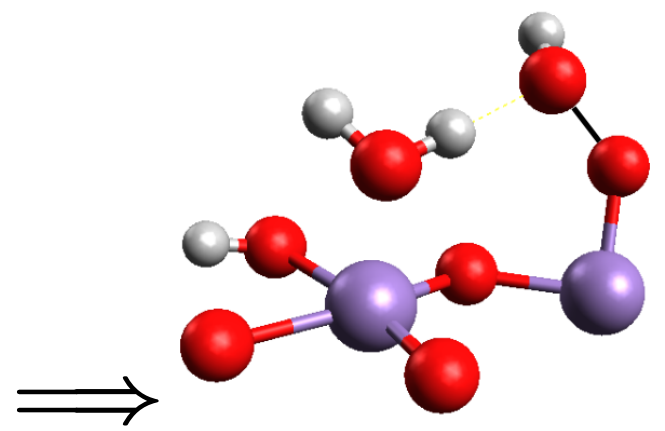

(b)

Fig. 4.20: Molecular representation of the unit cell. (a) Water interface and two different species of ${ }^{*} \mathrm{O}_{2} \mathrm{H}_{2}$ and ${ }^{*} \mathrm{OH}_{2}$, and (b) removing proton from hydrogen peroxide ion and making * $\mathrm{OOH}$ species.

\subsubsection{Third Proton-Electron Transfer}

In the third PET, the following reaction mechanism is suggested:

$$
{ }^{*} \mathrm{OOH}+h^{+} \rightarrow^{*} \mathrm{O}_{2}^{-}+\mathrm{H}^{+}
$$

It forms a Mn-O-O species and an $\mathrm{H}_{3} \mathrm{O}^{+}$ion. The O2-O3 distance is $1.331 \AA$ and thus lies relatively midway between the distances of molecular oxygen and hydrogen peroxide. The band gap of the system is $1.1 \mathrm{eV}$. This means that the hole migrates to the the ${ }^{*} \mathrm{OOH}$ species, where it leads to the formation of the superoxide ${ }^{*} \mathrm{O}_{2}$. The optimized geometry is shown in Fig 4.21.

The calculated DoS is shown in Fig. 4.22. The HOMO of the system are again the oxygen $p$ orbitals in the bulk $\mathrm{CaMnO}_{3}$ and small contribution from the water molecules. The LUMO is located at $\mathrm{O} 2$ and O3. It is a $\pi$-bond in the spin-down direction. The $\sigma$-bond, which was previously in focus, lies energetically above the conduction band. The $\pi$-bond is located in the bottom part of the conduction band with spin down direction. It makes band gap smaller. 


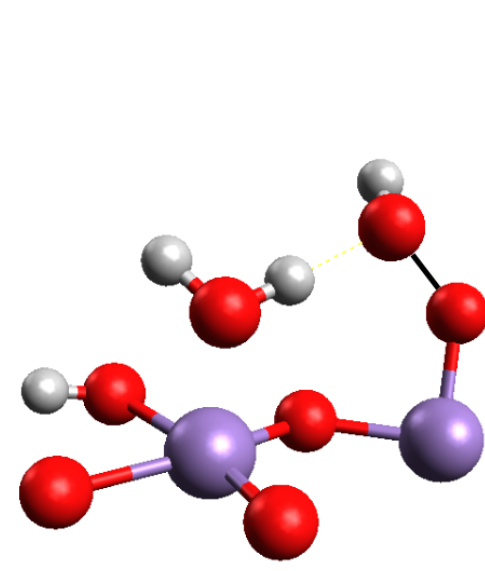

(a)
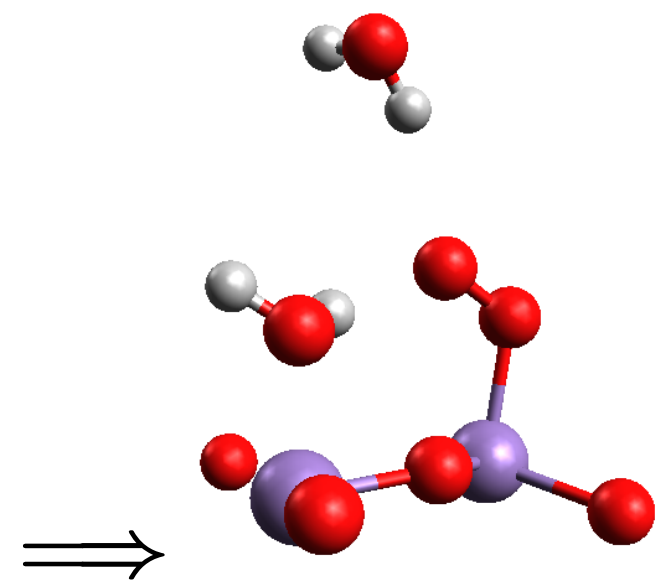

(b)

Fig. 4.21: Molecular representation of the unit cell. (a) Water interface and two different species of ${ }^{*} \mathrm{OOH}$ and ${ }^{*} \mathrm{OH}_{2}$, and (b) removing proton from ${ }^{*} \mathrm{OOH}$ and making $\mathrm{O}-\mathrm{O}$ bond.

\section{Deprotonated hydroperoxyl}

The next step in the mechanism is the deprotonation of the hydroperoxyl radical. The most obvious candidate for deprotonation is the $\mathrm{H}_{3} \mathrm{O}^{+}$ion.

The calculated DoS of third proton transfer is shown in Fig. 4.23. The filled O- $p$ states ate located in the upper part of the valence band which is related to the $\pi$-bond between Mn- $d$ and O- $p$ on the surface. The empty O- $p$ states overlap energetically with the empty Mn- $d$ stares which is corresponding antibonding of Mn- $d$ and O- $p$ on the surface. These two orbitals are shown in Fig. 4.23. To proceed further, we need to add a forth hole in proximity of adsorbed $\mathrm{O}_{2}$.

\subsubsection{Fourth Proton-Electron Transfer}

In the fourth PET, we have the following reaction mechanism:

$$
{ }^{*} \mathrm{O}_{2}^{-} \rightarrow^{*}--\mathrm{O}_{2}
$$

In the fourth PET, a surface-trapped hole $\left(\mathrm{h}^{+}\right)$reacts with an adsorbed superoxo species to break a hydrogen bond and create a $\mathrm{O}_{2}$ molecule. On $\mathrm{CaMnO}_{3}$ surface, next to the superoxo species, water adsorbs at the 5 -fold Mn surface site. Two possible sites exist for a deprotonation: i.e., one hydrogen which is lost its hydrogen bond and a water molecule which wants to replace by $\mathrm{O}_{2}$ molecule. In the both cases, we will reach to the same atomic structure. We thus focused on a hole localized at superoxo species and, assuming the proton to be transferred from the adsorbed water next to the $\mathrm{O}_{2}$ molecule. In the end, a radical $\mathrm{OH}$ and an adsorbed water are formed on the $\mathrm{CaMnO}_{3}$ surface. 


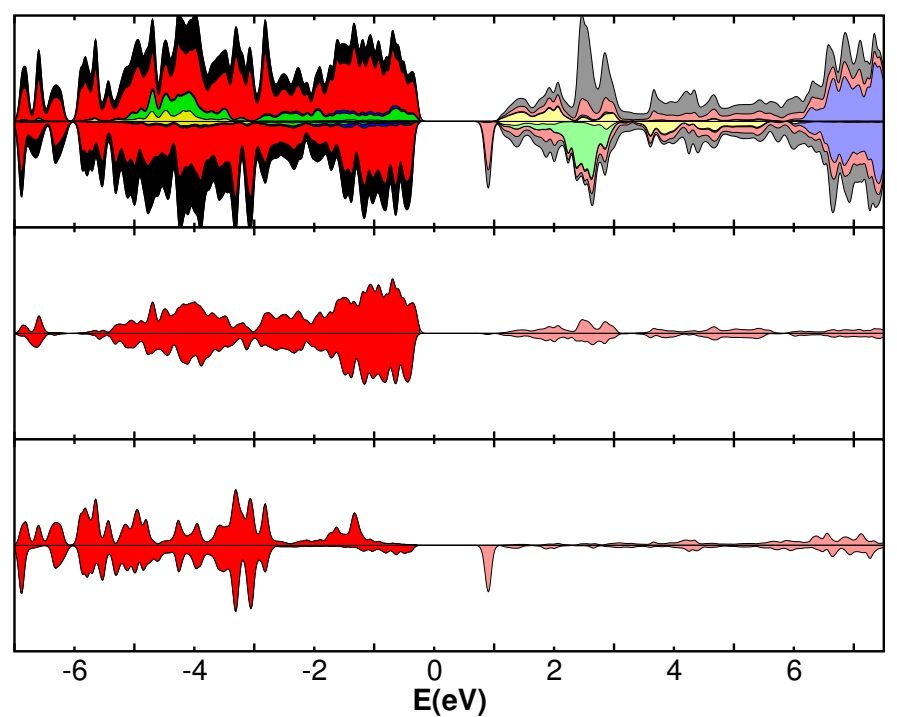

(a)

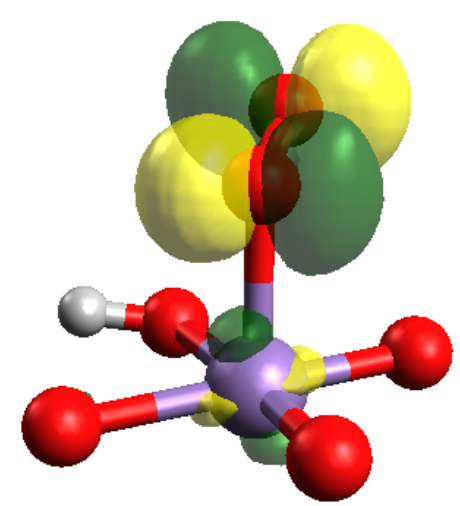

(b)

Fig. 4.22: (a) DoS of the third hole doped structure. The three graphs indicate different DoS. The top figure shows the total DoS (black envelope) with the projected DoS for O- $p$ (red), Mn- $t_{2 g}$ (green), Mn- $e_{g}$ (yellow), and Ca- $d$ (blue). Projected DoS are stacked on top of each other. The two spin densities are shown with opposite sign. For the Mn ion, the projected DoS is consider with one majority spin direction. The graph in the middle shows the oxygen contribution in the bulk $\mathrm{CaMnO}_{3}$. The bottom graph shows the oxygen contribution in the water molecules. (b) Orbital representation of the state in the bottom part of the conduction band which is created by hole doping.

The ground state of oxygen is the triplet oxygen ${ }^{3} \mathrm{O}_{2}$ with a total spin of $S=1 \hbar$. For the present study only the triplet state are investigated. The main effect of the singlet states is that one of the $\pi$-bond is fully occupied while the other is vacant in both spin directions. The triplet oxygen is about $0.99 \mathrm{eV}$ more stable than the singlet state. The oxidation reactions were carried out with the equilibrium structures of the previous section. 


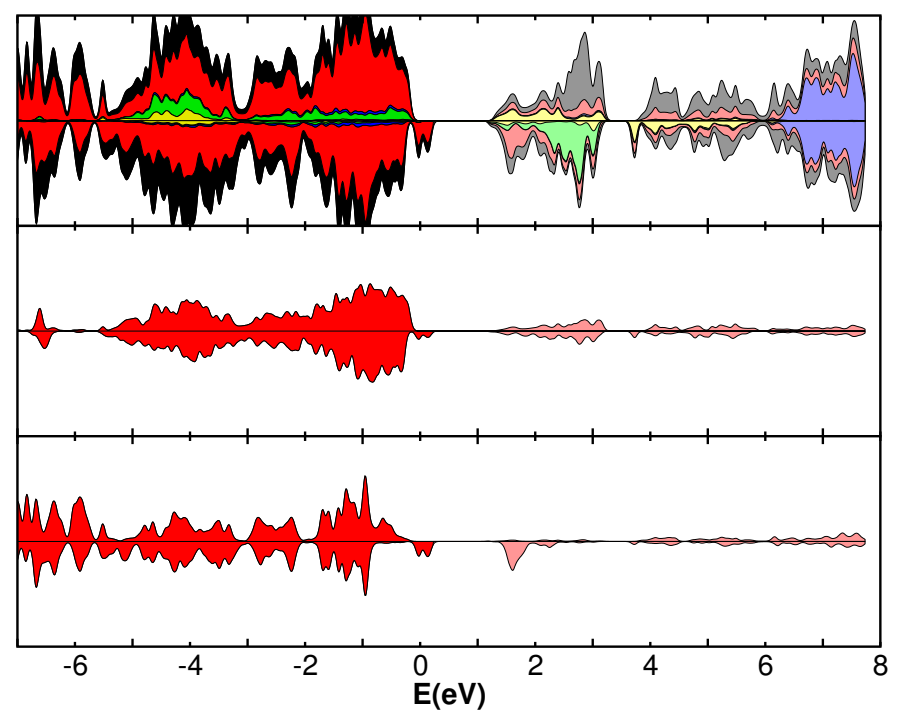

(a)

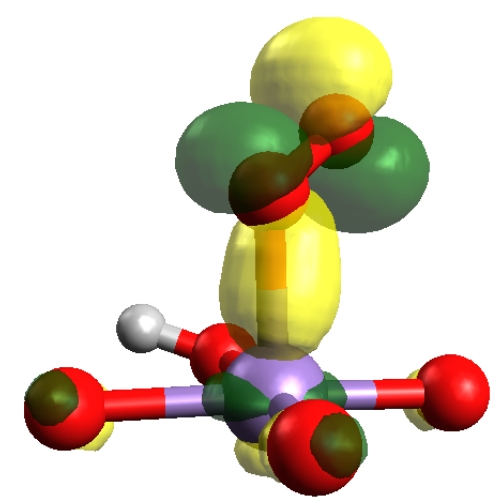

(b)

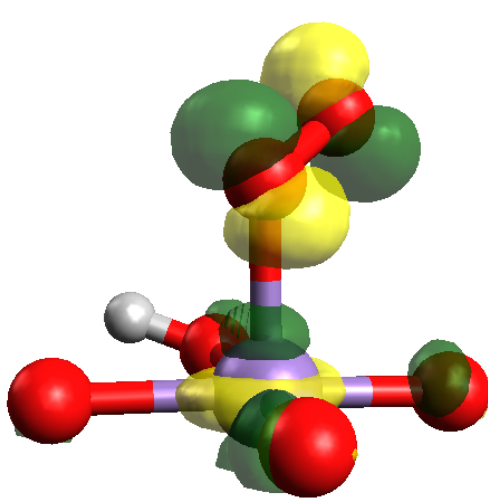

(c)

Fig. 4.23: (a) DoS of the third deprotonated structure. The three graphs indicate different DoS. The top figure shows the total DoS (black envelope) with the projected DoS for O- $p$ (red), Mn- $t_{2 g}$ (green), Mn- $e_{g}$ (yellow), and Ca- $d$ (blue). Projected DoS are stacked on top of each other. The two spin densities are shown with opposite sign. For the Mn ion, the projected DoS is consider with one majority spin direction. The graph in the middle shows the oxygen contribution in the bulk $\mathrm{CaMnO}_{3}$. The bottom graph shows the oxygen contribution in the water molecules. (b) Orbital representation of the state in the top of the valence band which is created by deprotonation. (c) Orbital representation of the state in the bottom part of the conduction band which is created by deprotonation.

The two right water molecules in the upper layer are rotated. In addition, the relative distances change greatly. Neither O1 nor O2 or O3 get hydrogen bonds. The water molecule just above $\mathrm{O} 3$ is only $2.52 \AA$ away and could, if it rotates, form a hydrogen bond to O3. It seems, however, as if it was energetically more favorable not to include the adsorbed oxygen in the hydrogen-bonding network. One possible reason is the low polarity 
of molecular oxygen. The Mn-O distance is $2.27 \AA$, the O-O distance is $1.23 \AA$. It should be noted that the moved network does not mean that the right side of the slab has no contact with the water. It is a periodic system so that the part of the water layer that projects beyond the slab to the left is actually over the right side of the adjacent slab.

The LUMO of the system describes four (two in the layer above two in the layer below the slab) degenerate $\pi$-bond in the spin-down direction. This actually identifies the oxygen species as ${ }^{3} \mathrm{O}_{2}$.

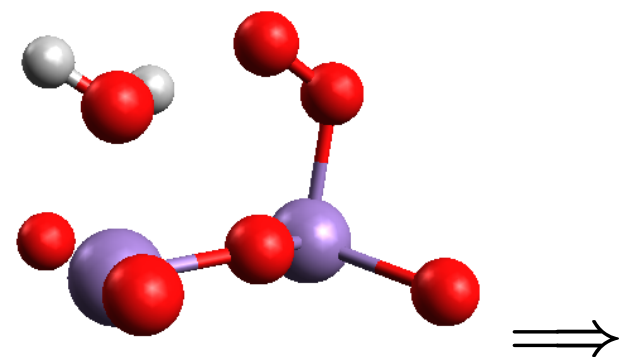

(a)

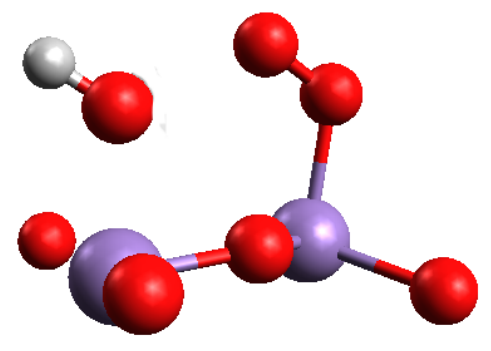

(b)

Fig. 4.24: Schematic representation of the unit cell with labels for the important surface atoms. (a) Water interface and two different species of ${ }^{*} \mathrm{OH}_{2}$ and ${ }^{*} \mathrm{O}_{2}$, and (b) breaking down a hydrogen bond and removing proton from water molecule and making $\mathrm{OH}$ group.

The calculated DoS is shown in Fig. 4.25. The HOMO of the system are again the oxygen $p$ orbitals in the bulk $\mathrm{CaMnO}_{3}$ and small contribution from the water molecules. The LUMO is located at $\mathrm{O} 2$ and O3. It is a $\pi$-bond in the spin-down direction. The $\sigma$-bond, which was previously in focus, lies energetically above the conduction band. The $\pi$-bond is located in the bottom part of the conduction band with spin down direction. It makes band gap smaller.

\section{Deprotonated water molecule}

For completeness, the last deprotonation is needed. The most obvious candidate is the nearest neighbor $\mathrm{H}_{2} \mathrm{O}$ to the $\mathrm{O}_{2}$. The $\mathrm{O}-\mathrm{O}$ bond is formed and it can be released from the surface. There was a hydrogen bond between superoxo species on the surface and the hydrogen atom from the absorbed water molecule. When the fourth hole introduced into the system, this hydrogen bond was broken. The best candidate for deprotonarion is the hydrogen ion which is lost its hydrogen bond. Another possibility is to remove a proton from the water molecule which will be replaced instead of $\mathrm{O}_{2}$ molecule on the surface. In both cases, we reach to the same atomic position. Ultimately, the $\mathrm{O}_{2}$ molecule will be replaced by the water molecule and the surface is covered by a water molecule and one hydroxide group.

The calculated DoS of forth proton transfer is shown in Fig. 4.25. The filled O- $p$ states are located in the upper part of the valence band which is related to the $\pi$-bond between 


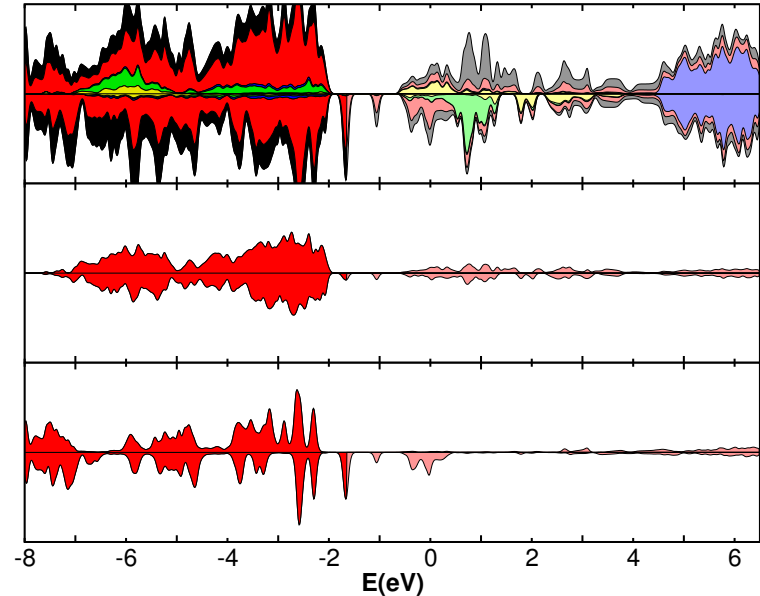

(a)

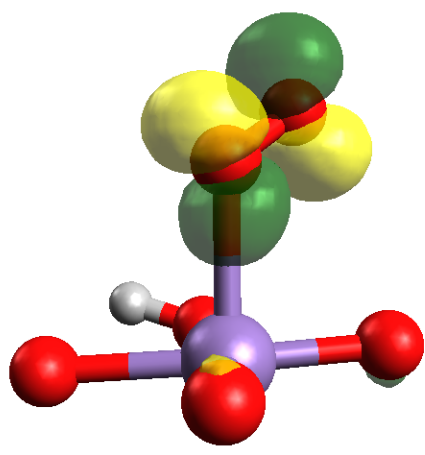

(c)

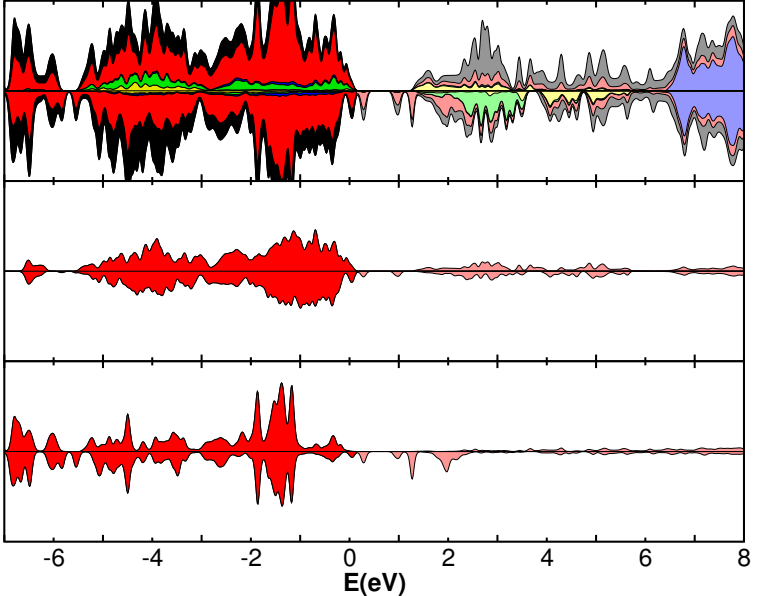

(b)

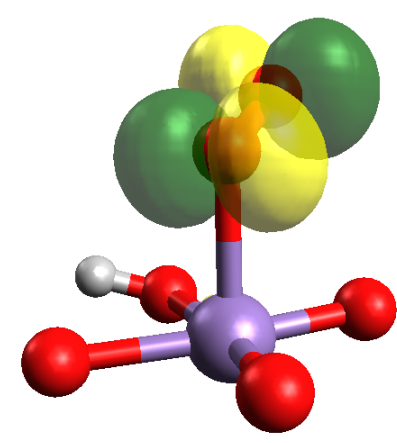

(d)

Fig. 4.25: DoS of the (a) fourth hole doped structure and (b) fourth deprotonated structure. The three graphs indicate different DoS. The top figure shows the total DoS (black envelope) with the projected DoS for O- $p$ (red), Mn- $t_{2 g}$ (green), Mn- $e_{g}$ (yellow), and Ca- $d$ (blue). Projected DoS are stacked on top of each other. The two spin densities are shown with opposite sign. For the Mn ion, the projected DoS is consider with one majority spin direction. The graph in the middle shows the oxygen contribution in the bulk $\mathrm{CaMnO}_{3}$. The bottom graph shows the oxygen contribution in the water molecules. (c), (d) Orbital representation of the states in the bottom part of the conduction band which are created by fourth deprotonation.

Mn- $d$ and $\mathrm{O}-p$ on the surface. The empty O- $p$ states overlap energetically with the empty Mn- $d$ stares which is corresponding antibonding of Mn- $d$ and O- $p$ on the surface. These two orbitals are shown in Fig. 4.23. 


\subsubsection{Calculation of the OER energy profile}

To calculate the energy profile of photo-/electro-chemical water oxidation, the reaction energies are calculated as follows:

$$
\begin{aligned}
& \Delta E_{A}=E\left({ }^{*} O_{2} H_{2}\right)-E(\text { system })+\frac{1}{2} E_{H_{2}} \\
& \Delta E_{B}=E\left(^{*} O O H\right)-E\left({ }^{*} O_{2} H_{2}\right)+\frac{1}{2} E_{H_{2}} \\
& \Delta E_{C}=E\left(^{*} O O\right)-E\left(^{*} O O H\right)+\frac{1}{2} E_{H_{2}} \\
& \Delta E_{D}=E\left(-O_{2}\right)-E\left(^{*} O O\right)+\frac{1}{2} E_{H_{2}}
\end{aligned}
$$

We adopt the first and simplest model, which has no temperature, pressure and zero point energy (ZPE) contributions to the reaction energy. Temperature increasing from 0 to $298 \mathrm{~K}$ has little impact on the enthalpy and the changes are normally neglected. We are only considering reaction steps involving charge transfer. We consider a process where oxygen molecule are formed by an associative mechanism on the catalyst.

On the aqueous $\mathrm{CaMnO}_{3}$ (001) interface the intermediate structures along with the calculated energies are shown in Fig. 4.26. Starting with an initial structure of watercovered surface, water is dissociated at the surface hydroxide forming a peroxide group. Next, the proton is removed, resulting in ${ }^{*} \mathrm{OOH}$ on the Mn-ion surface site. Finally upon the one more deprotonation, $\mathrm{O}_{2}$ desorbs at the surface.

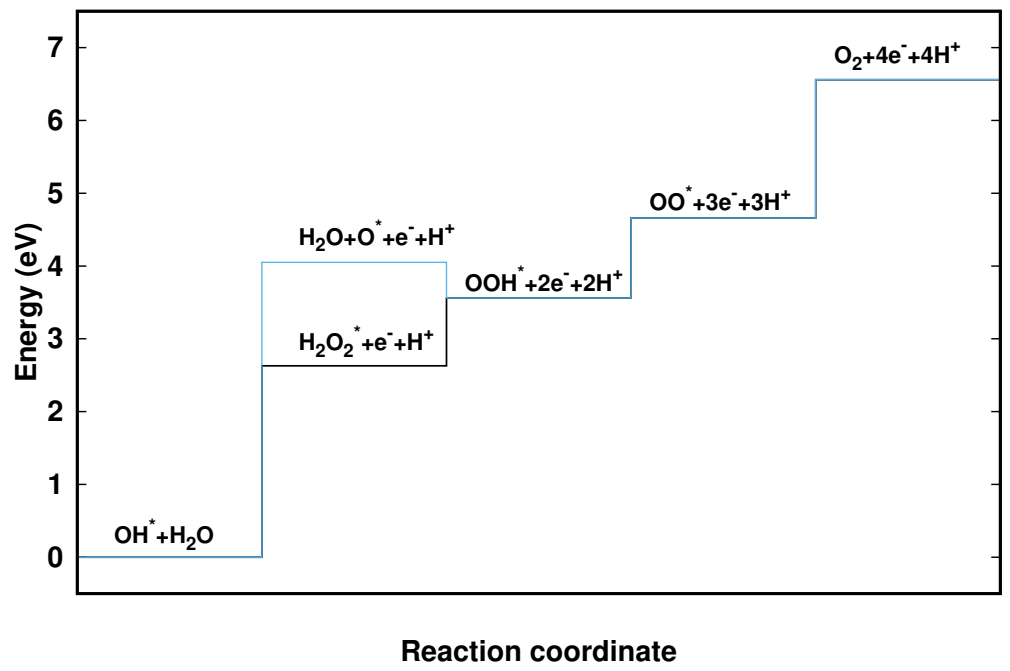

Fig. 4.26: The energies of the intermediates on water covered $\mathrm{CaMnO}_{3}$ surface. The reaction steps are uphill in energy. The black line shows the hemibonding structure while the blue line represents the bare oxygen on the surface. 
The largest energy difference is found for the first step, which is splitting the water molecule. This means that the first step requires more energy. The higher energy of bare oxygen shows that hydrogen bonds between the water molecules tend to stabilize ${ }^{*} \mathrm{OH}$ and ${ }^{*} \mathrm{OOH}$ slightly compared to the situation with ${ }^{*} \mathrm{O}$.

Note that this approach only accounts the elementary reaction steps and no barriers are included. This means that the method presented here is necessary but not sufficient.

\subsection{Summary}

Overall, the elementary processes of photolytic water splitting are investigated. On the one hand, the analysis of the water dimer provided an understanding of the molecular orbitals of a hydrogen bond, and, on the other hand, the ionization rotation could be applied in the formation of stable $\sigma$ bonds between the oxygen atoms.

Our results show that the hydroxide groups and water molecules are involved in the OER on $\mathrm{CaMnO}_{3}$ (001). The hole is localized between the hydroxide and water molecule and broken the hydrogen bond to form a hemibonding structure. Water molecule releases a proton on the outer layers of water. While this species is qualitatively similar to the surface peroxide intermediate, it needs one more hole to make peroxide species. ${ }^{*} \mathrm{OOH}$ can readily form from peroxide via the electron proton transfer. Once ${ }^{*} \mathrm{OOH}$ is formed, generation of *OO via nucleophilic attack of a water molecule is favorable. Our results thus suggest that the $\mathrm{O}$ source for the $\mathrm{O}_{2}$ molecule is determined by the ${ }^{*} \mathrm{OH}$ species. The OER pathway on the $\mathrm{CaMnO}_{3}$ surface is more favorable through the hemibonding structure compared to the bare oxygen which is proposed in the earlier studies.

Simultaneously, we have been explored the surface oxygen participation in the OER pathway. Hole localization between the ${ }^{*} \mathrm{OH}$ and $\mathrm{O}_{\text {surf }}$ is not favorable. Corrosion, which is chemically similar to the water splitting reaction, is not likely for $\mathrm{CaMnO}_{3}$ (001) surface. 


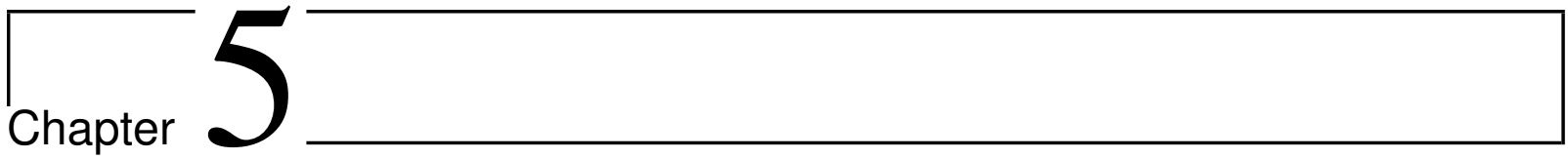

\section{Role of defects in titanium dioxide}

This chapter is devoted to the theoretical description of the quasiparticle defect states in rutile titanium dioxide $\left(\mathrm{TiO}_{2}\right)$. Rutile is considerably simpler than the manganites due to the empty $d$-orbitals. In order to gain insight into the corrosion reactions, defects related to oxygen vacancies and hydrogen have been investigated in rutile for different Fermilevels. The defect concentration in rutile next to a buried interface with $\mathrm{Pd}$ interface can be controlled by loading it with hydrogen. This has been done experimentally using energy-loss near edge structure (ELNES). Within $2 \mathrm{~nm}$ from the interface hydrogen-induced changes of the spectra when the hydrogen partial pressure reaches $10 \mathrm{~Pa}$. In order to rationalize the observations, we compared defects related calculations with experimental measured ELNES. The nature of the electronic structure of the defects have been explored in detail. The calculated defect concentrations and the defect electronic structure explain experimental EEL spectra measured under hydrogen loading. We find that oxygen vacancy defect considered have charging levels in a narrow energy window, which is the expected Fermi-level pinning range. Negative defects are not produced for both PBE and PBE0r density functionals. In the following, the historical perspective and experimental data will be reviewed at the beginning of this chapter. Next, we try to analysis the electronic and structural properties of defect related systems. We provide a mechanistic picture of the underlying chemical processes.

\subsection{Importance of $\mathrm{TiO}_{2}$}

In the recent years, $\mathrm{TiO}_{2}$ has been attracted lots of interest due to the numerous applications in the science and technology [150, 151]. These applications are in pigment, gas sensors, catalysts, and electrolytes which are mainly connected with its optical and photocatalytic properties $[152,153,154,155,156,157,158,159,160,161,162]$. Among all these items, $\mathrm{TiO}_{2}$ has been studied extensively from 1970s because of the discovery of photocatalytic activity [163]. This material also can be used as a support for metal catalysts and acts as an electrode coating in photoelectrolysis cells [164] to enhance the efficiency of 
electrolytic splitting of water. This material is chemically stable, nontoxic and compatible with the environment. The band gap of $\mathrm{TiO}_{2}$ is in the ultraviolet region of solar spectrum of $3.1 \mathrm{eV}$ in rutile and $3.2 \mathrm{eV}$ in anatase. The main component of the conduction band is the Ti-3d orbital and the electronic structure has the character of a strongly correlated system.

Titanium dioxide $\left(\mathrm{TiO}_{2}\right)$, has been popular and promising materials in the photocatalytic applications for decades due to its strong oxidizing power, non-toxicity, and high reactivity $[163,165,166,157]$. However, $\mathrm{TiO}_{2}$ only absorbs the ultraviolet portion of sunlight because of the large band gap (more than $3.0 \mathrm{eV}$ ) which is almost $5 \%$ of the solar radiation. In order to produce more efficient absorption, different methods for reducing the band gap of $\mathrm{TiO}_{2}$ have been proposed $[167,168]$. These strategies include introducing metallic/non-metallic ion impurities [169, 170, 171] into the system, self-doping [172], and introducing disorders [173] with simultaneous dopant incorporation. In a recent approach, Chen et al. [168] have realized the effect of hydrogenation of $\mathrm{TiO}_{2}$ nano-crystals. They have engineered the disorder of $\mathrm{TiO}_{2}$ nano-crystals by exposing them to hydrogen $(\mathrm{H})$ in a wide range of pressures and they reported the improvement of photocatalytic activity. Recent studies $[174,175,176,177]$ have discussed defects in hydrogenated $\mathrm{TiO}_{2}$ and showed that the favourable absorption sites for $\mathrm{H}$ atoms are oxygen $(\mathrm{O})$ vacancies and, in absence of vacancies, $\mathrm{H}$ interstitially makes a weak $\mathrm{O}-\mathrm{H}$ bond, and becomes positive.

Although great efforts have been made to understand the hydrogen treatment, but atomic and electronic structure in hydrogenated $\mathrm{TiO}_{2}$ has remained elusive. Many of local density approximation (LDA) and generalized gradient approximation (GGA) calculations are available for the defects states of $\mathrm{TiO}_{2}$ [178, 179, 180, 181]. However, because of the lack of self-interaction cancellation, LDA and GGA are not able to estimate band gaps and localization of defect states $[182,183]$. To correct these problems several methods including $\mathrm{LDA}+\mathrm{U}[184,185]$, B3LYP [186], HSE [187], and sX [188] functionals have been considered. $\mathrm{LDA}+U$ method works better for open shell systems and B3LYP overestimates the band gap. HSE and sX functionals used to study the influence of O-vacancy and dopants but they did not treat the interstitial $\mathrm{H}$ atoms. It should be noted that hybrid density functionals are a better method for this case, because they combine a much improved description of the exchange energy and, as generalized Kohn-Sham functionals, they can be used for total energy minimization and structure relaxation. This is because of this fact: the defect localization in $\mathrm{TiO}_{2}$ comes not only from the electronic state, but also the interaction of electronic states with lattice distortions (a polaronic effect) are involved.

Here we investigate the H-doped, O-vacancy, and hydrogen complex with the oxygen vacancy in bulk rutile $\mathrm{TiO}_{2}$ using the $\mathrm{PBE}$ and the local hybrid (PBE0r) functional, which is derived from the PBE0 [64] functional by mapping the Kohn-Sham wave functions onto localized tight-binding orbitals. In this case, only the onsite exchange terms of the exchange correction are included. This method improves the band gap of $\mathrm{TiO}_{2}$ by $0.3 \mathrm{eV}$ and also stabilizes the defect states in the band gap. We studied the different defects to explore the competition between the various ordering phenomena. Interstitial $\mathrm{H}$ and $\mathrm{H}$ with $\mathrm{O}-$ vacancy have shown more stability comparison to other defects in $\mathrm{TiO}_{2}$ and interstitial $\mathrm{H}$ 
is of interest in the absence of vacancies. By combination of the experiment and Theory for bulk $\mathrm{TiO}_{2}$, experimental EELS data are interpreted. We have extended our calculations to clarify the physics behind the H-doped system and therefore it can open up new chance in clean energy techniques. The comparison of the PBE and PBE0r functional is included.

\subsection{Rutile titanium dioxide}

Titanium dioxide has three natural polymorphs as the well-known minerals rutile, anatase and brookite which is shown in Fig. 5.1. Rutile is the most abundant and contains around 98\% titanium dioxide. The metastable anatase and brookite phases convert irreversibly to the equilibrium rutile phase upon heating above temperatures in the range $600-800{ }^{\circ} \mathrm{C}$.

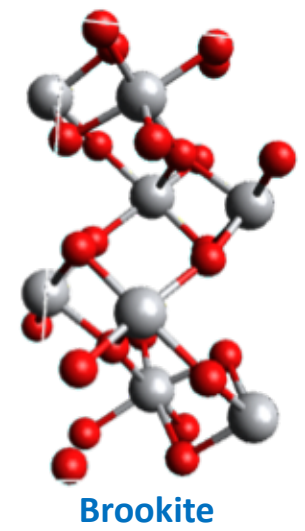

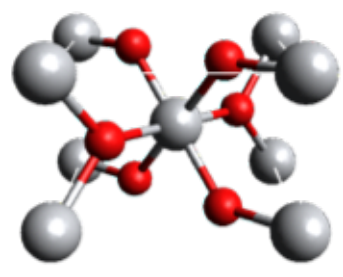

Rutile

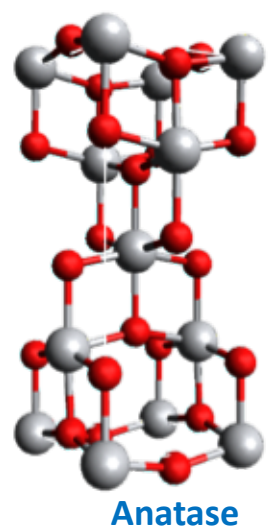

Anatase

Fig. 5.1: Three natural polymorphs of $\mathrm{TiO}_{2}$. The red spheres represent the formal $\mathrm{O}^{2-}$ and the gray spheres shows the formal $\mathrm{Ti}^{4+}$ ions.

$\mathrm{TiO}_{2}$ in rutile phase has a tetragonal unit cell which is represented by the $P 4_{2} / \mathrm{mnm}$ space group as shown in Fig. 5.1. Each Ti ion surrounded by six oxygens in the octahedral environment. The $\mathrm{TiO}_{6}$ octahedron possesses a formal valence state of $4+$ for $\mathrm{Ti}$ ion. The lattice parameters of the unit cell are $a=b=4.5937 \AA$ and $c=2.9581 \AA$ [189]. In the ideal octahedron Ti-O distances are equal, while in rutile structure one of the O-Ti-O axis is elongated. Orthogonal to this elongated axis the Ti-O distances are the same but four $\mathrm{O}$ ions in this $\mathrm{TiO}_{4}$-plane have two different distances which make a rectangular shape instead of a squared one. Thus, the octahedron has an orthorhombic distortion [190] with the corresponding point group of $\mathrm{D}_{2 \mathrm{~h}}[190,191]$.

\subsubsection{Shape of the Ti $L$ edge}

The Ti $L$ edge spectroscopy is an experimental technique to study the electronic structure of $\mathrm{Ti}$ atoms. This method measures x-ray absorption originated from an excitation of Ti- $2 p$ state electrons into the unoccupied $d$-states in the conduction band which creates a 
peak called the $L$ edge. According to the selection rules, this transition is electric-dipole allowed. Since the Ti- $2 p$ states are split by spin orbit coupling ( $2 p_{3 / 2}$ and $2 p_{1 / 2}$ state) the two related $L_{3}$ and $L_{2}$ edges exist [191] as indicated in Fig. 5.2. The Ti ion has an octahedral environment resulting in transitions to the $t_{2 g}$ and $e_{g}$ sets. Therefore, the energy splitting in $t_{2 g}$ and $e_{g}$ is present in the Ti $L$ ELNES. The $L_{3}$ edge shows the $a\left(t_{2 g}\right)$ and $b / b^{\prime}$ $\left(e_{g}\right)$ peaks while the $L_{2}$ edge shows the $c\left(t_{2 g}\right)$ and $d\left(e_{g}\right)$ peaks. The splitting of the $b / b^{\prime}$ in $b$ and $b^{\prime}$ is typically explained by the picture of the orthorhombic distortion $\mathrm{D}_{2 \mathrm{~h}}$ of the octahedron in rutile $\mathrm{TiO}_{2}$ [190]. However, in the present study it is sufficient to distinguish the features in the Ti ELNES and DoS in $\mathrm{O}_{\mathrm{h}}$ symmetry relative to the $t_{2 g}$ and $e_{g}$ states.

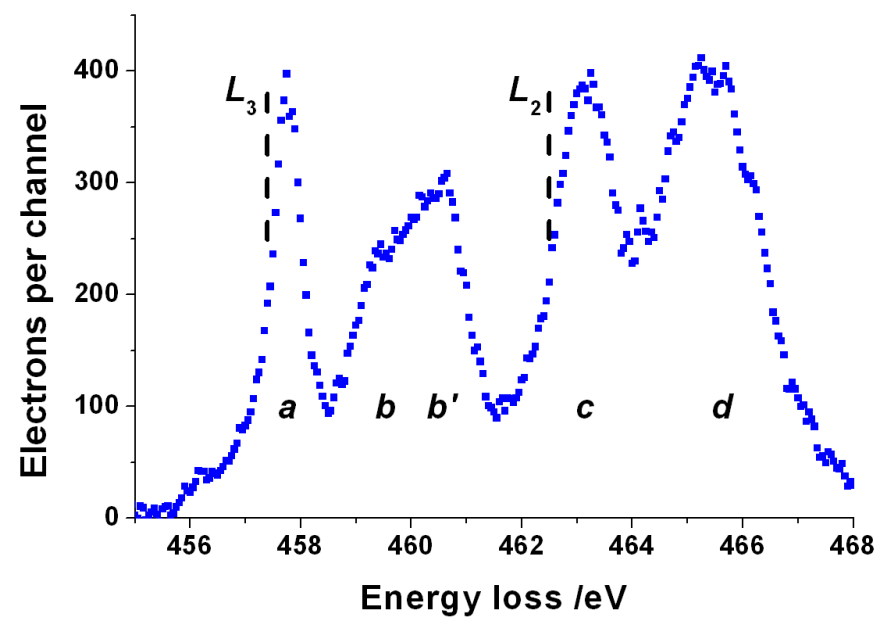

Fig. 5.2: $\mathrm{Ti} L_{3,2}$ ELNES of rutile $\mathrm{TiO}_{2}$. Indicated are the four white lines $\left(a, b / b^{\prime}, c, d\right)$ as well as the fine-structure splitting $\mathrm{d} E\left(b^{\prime}-a\right)$ as an example for an evaluation parameter. With kind approval of M. Bongers and A. Pundt [192].

A change in the shape of the Ti $L$ ELNES represents changes in the electronic structure of the conduction band [191]. This is reported by Stoyanov et al. [191] for different stoichiometric phases of Titanium oxides. They have shown that the $L_{2} / L_{3}$ ratio depends on the oxidation state and it decreases with decreasing the oxidation state. Moballegh and Dickey [193] have presented a decrease of the Ti $L$ edge onset (chemical shift) with decreasing oxygen content.

The symmetry labels associated with the Ti $L$ peaks will be discussed next.

\subsubsection{Octahedral symmetry}

In the ideal octahedral symmetry $\left(\mathrm{O}_{\mathrm{h}}\right)$, the $d$-orbitals split into two sets namely the triply degenerate $t_{2 g}$ and the doubly degenerate $e_{g}$ states. This is so-called crystal-field splitting [190]. Due to the orthorhombic distorted octahedron in $\mathrm{TiO}_{2}$, the symmetry lowered and it makes a further energy splitting that is discussed next.

The orthorhombic distorted octahedron with the corresponding $\mathrm{D}_{2 \mathrm{~h}}$ point group yields to an additional energy splitting as summarized in Tab. 5.1. The degenerated $t_{2 g}$-states 
of the octahedral point group further into a $b_{2 g}$, an $a_{g}$ and a $b_{3 g}$ state in the setting of $\mathrm{D}_{2 \mathrm{~h}}$ [190]. Also the $e_{g}$ states of the octahedral point group are split into two, namely the $a_{g}$ and $b_{1 g}$ states [190].

Table 5.1: $d$-orbitals and their symmetry label in the setting of the ideal octahedral $\left(\mathrm{O}_{\mathrm{h}}\right)$ and the orthorhombic distorted octahedral $\left(\mathrm{D}_{2 \mathrm{~h}}\right)$ symmetry group of rutile. The orbitals refer to the local coordinate system with the $z$-axis aligned with the elongated O-Ti-O axis of the octahedron and the $x$ - and $y$-axis are aligned with the two shorter octahedral axes, respectively. The white lines in the $\mathrm{Ti} L_{3,2}$ edge are given that are associated with the energy states resulting from the two octahedral symmetries.

\begin{tabular}{lllll}
\hline \hline$d$-orbital & $\begin{array}{l}\text { symmetry } \\
\text { label in } \mathrm{O}_{\mathrm{h}}\end{array}$ & symmetry & $L_{3}$ white & $L_{2}$ white \\
& $t_{2 g}$ & $b_{2 g}$ & $a$ & line \\
\hline$d_{x z}$ & $t_{2 g}$ & $a_{g}$ & $a$ & $c$ \\
$d_{y z}$ & $t_{2 g}$ & $b_{3 g}$ & $a$ & $c$ \\
$d_{x y}$ & $t_{2 g}$ & $a_{g}$ & $b$ & $d$ \\
$d_{3 z^{2}-r^{2}}$ & $e_{g}$ & $b_{1 g}$ & $b^{\prime}$ & $d$ \\
$d_{x^{2}-y^{2}}$ & $e_{g}$ &
\end{tabular}

\subsubsection{Electronic structure of rutile}

The density of states (DoS) for rutile $\mathrm{TiO}_{2}$ calculated from the PBE functionals is shown in Fig. 5.3. The $t_{2 g}$-like and $e_{g}$-like states (referring to the octahedral symmetry as discussed in Tab. 5.1 ) are clearly separated. The $e_{g}$ states are the $d$-orbitals with lobes pointing towards the oxygen neighbors. While forming Ti-O antibonds, the $e_{g}$-like states are lifted up by approximately $3 \mathrm{eV}$ relative to the $t_{2 g}$-like states. This is the crystal-field splitting. The corresponding Ti-O bonding states are located in the lower part of the oxygen valence band. The $t_{2 g}$-like orbitals form much weaker $\pi$-bonds and antibonds and their crystal-field splitting is therefore smaller.

As seen in Tab. 5.2, the calculated band gap is with $2.203 \mathrm{eV}$ smaller than the experimental value of $3.05 \mathrm{eV}[194]$. Conventional functionals such as PBE underestimate the band gap by approximately $1 \mathrm{eV}$. The PBE0r functional with the correct parameters open up this band-gap by $0.3 \mathrm{eV}$. For these calculations, the width of the bandgap is crucial for stabilizing the electron-rich charge state of defects. In standard DFT calculations only the most positive charge state is stable, while the charging levels shift from the conduction band into the band gap when hybrid functionals are used. This effect is essential for the description of the band bending on hydrogen loading. If the band gap is too small, the corresponding electron levels become resonances in the conduction band and thus donate their electron.

The calculated structure parameters and heats of formation given in Tab. 5.2. The PBE and PBE0r functional with our choice of parameters are considered. 


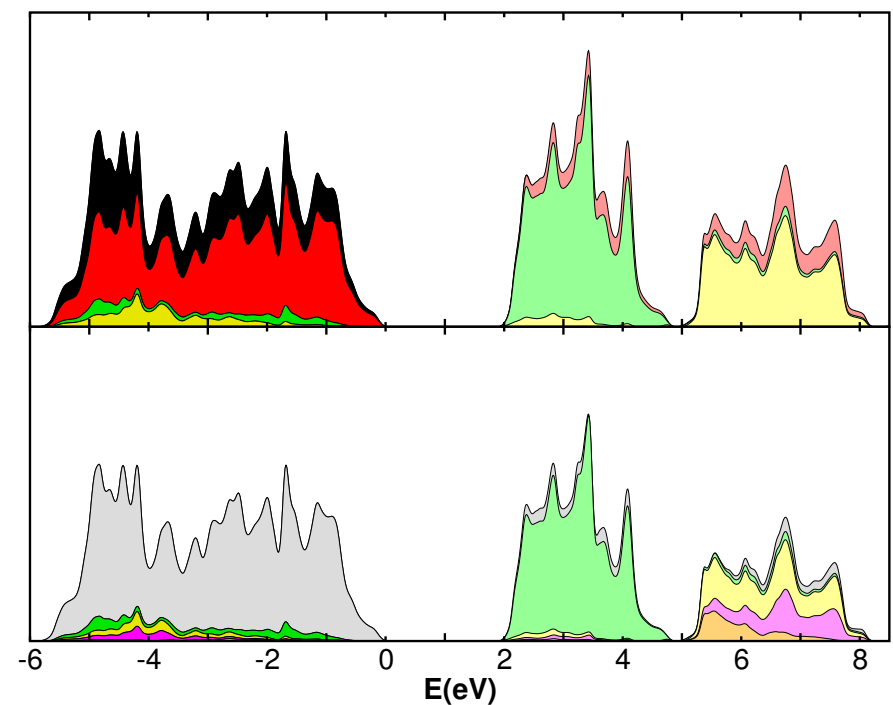

Fig. 5.3: Density of states (DoS) for rutile $\mathrm{TiO}_{2}$. The total DoS is given in black and gray, respectively. The projected DoS are shown in red for oxygen, in green for $t_{2 g}$-like and yellow for $e_{g}$-like Ti- $d$ orbitals. Unoccupied states are drawn in a lighter color than the filled states. The energy zero is set to the top of the valence band. The bottom graph treats the perfect oxygen site as a defect with symmetry adapted orbitals. Hence, the $A_{1}^{\prime}$ (orange), $E^{\prime}$ (magenta), $\delta$ - $e_{g}$ (yellow) and $t_{2 g}$-delta (green) orbitals are shown as the sum of the corresponding three Ti neighbors of the oxygen site. These symmetry adapted orbitals are defined in Fig. 5.5.

Table 5.2: Lattice parameters $a$ and $c$, internal parameter $u$ [195] and band gaps of rutile from the PBE method, the PBE0r method and experiments [195, 196, 189].

\begin{tabular}{lccc}
\hline \hline Quantity & PBE & PBE0r & Experiment \\
\hline$a(\AA)$ & 4.562 & 4.504 & 4.593 \\
$c / a$ & 0.644 & 0.644 & 0.644 \\
$u / a$ & 0.302 & 0.303 & 0.305 \\
band gap $(\mathrm{eV})$ & 1.905 & 2.203 & 3.05 \\
heat of formation $(\mathrm{eV})$ & -8.58 & -8.90 & -9.74 \\
\hline \hline
\end{tabular}

\subsection{EELS experiments on $\mathrm{Pd} / \mathrm{TiO}_{2}$ upon H-exposure}

Palladium $(\mathrm{Pd})$ is often used as metal partners at the metal $/ \mathrm{TiO}_{2}$ interface, because they are highly active in hydrogenation reactions [197], and they allow for dissociative chemisorption of $\mathrm{H}_{2}$ molecules at their surfaces. Pd is a well-studied catalyst but also a system for hydrogen ad- and absorption [198, 199, 200]. Hydrogen solves on interstitial sites in the $\mathrm{Pd}$ lattice in the $\mathrm{Pd}-\mathrm{H} \alpha$-phase and in the $\mathrm{Pd}-\mathrm{H}$ hydride phase [201, 202]. The solubility of $\mathrm{H}$ depends on the hydrogen chemical potential [203]. It is, for thin films, also affected 
by the film's microstructure [204, 205, 206, 207] and the mechanical stress state [208]. For low chemical potentials, the hydrogen related mechanical stress is small [209]. For high chemical potentials of $\mathrm{H}$, the mechanical stress can be in the GPa-range[205].

The room-temperature kinetics of $\mathrm{H}$ in $\mathrm{Pd}$ is fast [210]. This allows for fast equilibration and, further, to fast transport of atomic hydrogen to the $\mathrm{Pd} / \mathrm{TiO}_{2}$ interface. $\mathrm{H}$ affects the electrical behavior of the Schottky contact $[211,164,212]$. The established picture is that the atomic hydrogen diffuses through the $\mathrm{Pd}$ to the interface where it spills over into the $\mathrm{TiO}_{2}$ [164]. In the $\mathrm{TiO}_{2}$ it acts like a dopant and thus changes the Schottky barrier thickness and/or height. Irreversible changes of the conductivity of hydrogen loaded $\mathrm{Pd} / \mathrm{TiO}_{2}$ hint on the presence of stable hydrogen-oxygen vacancy complexes [212].

Here experimentally, we investigated hydrogen related changes in the electronical density of states (DoS) of $\mathrm{TiO}_{2}$ in the nanometer vicinity to the $\mathrm{Pd} / \mathrm{TiO}_{2}$ interface [192]. To experimentally investigate these changes in the DoS of $\mathrm{TiO}_{2}$, in-situ scanning transmission electron microscopy (STEM) in combination with electron energy loss spectroscopy (EELS) is applied in an environmental TEM (ETEM). Changes of the Ti $L$ edge spectrum are observed at a hydrogen gas pressure of $10 \mathrm{~Pa}$, in the vicinity of the interface. The energy splitting is shown in Fig. 5.4. This effect sets in below $2 \mathrm{~nm}$ from the interface. The presence of this effect was confirmed on different positions close to the $\mathrm{Pd} / \mathrm{TiO}_{2}$ interface.

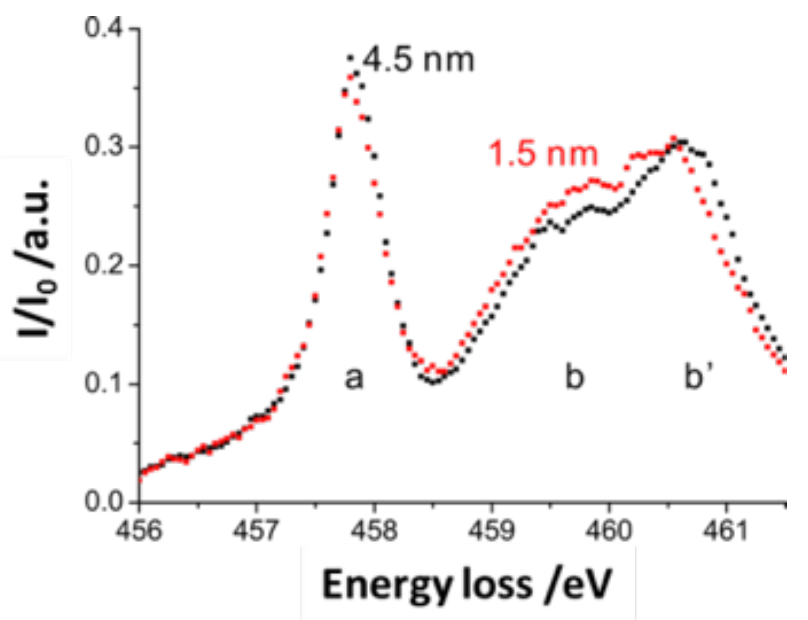

Fig. 5.4: $\mathrm{Ti} L_{3}$ ELNES shown for different distances to the interface, at a hydrogen partial pressures of $10 \mathrm{~Pa}$. The $1.5 \mathrm{~nm}$ spectrum is shown with red color and the $4.5 \mathrm{~nm}$ (bulk-like) spectrum is plotted in black. With kind approval of M. Bongers and A. Pundt [192].

We conduct local hybrid first-principles calculations based on density functional theory (DFT) on $\mathrm{TiO}_{2}$ containing differently charged defects to examine the changes in the corresponding DoS. These simulations offer detailed information about the nature of each defect. The combination of experiment and theory allows determining the relevant charged defects in the presence of hydrogen and the interface. Further, it provides the concentrations depth profile of the dominant charged defect. 


\subsection{Simulations}

In order to shed light on the experimental findings we performed calculations on hydrogen and oxygen-vacancy related defects. The nature of the defects is investigated using the projected density of states, wave function plots and the formation energies for different charge states.

\subsubsection{Triangle symmetry}

An incorporation of a defect into the $\mathrm{TiO}_{2}$ lattice results in local changes of the lattice structure, and in the local DoS. This will change the local symmetry of the lattice.

After inspecting the defect states, we find that some states are best characterized in terms of the point group of the defect center. Other states are best characterized according to the octahedral point group $\left(\mathrm{O}_{\mathrm{h}}\right)$ of the Ti-atoms neighboring the related defect center.

The electronic structure of the defects, in other words the partial DoS, can be understood by investigating the atoms involved and their three nearest Ti-neighbors. The Ti-neighbors form approximately an equilateral triangle, cf. Fig. 5.5. The $\mathrm{D}_{3 \mathrm{~h}}$ symmetry of this triangle will allow us to classify and rationalize the defect states. Specified $d$-orbitals of $\mathrm{Ti}$, namely the $d_{3 z^{2}-r^{2}}$ orbitals pointing into the center of the triangle, will be distinguished as $A_{1}^{\prime}$ and $E^{\prime}$, because this character dominates the corresponding wave functions. These Ti $d$-states states are the indicated orbitals in Fig. 5.5. For the other Ti $d$-states, we still refer to the octahedral point group and denote them as $t_{2 g}$ character and as $e_{g}$ orbital for the $d$-orbitals having $\delta$-bond character towards the triangle center. These states are denoted as $\delta-e_{g}$ for the $e_{g}$ orbital with $\delta$-type rotational symmetry about the axis towards the defect center, and as $t_{2 g}$ orbitals.
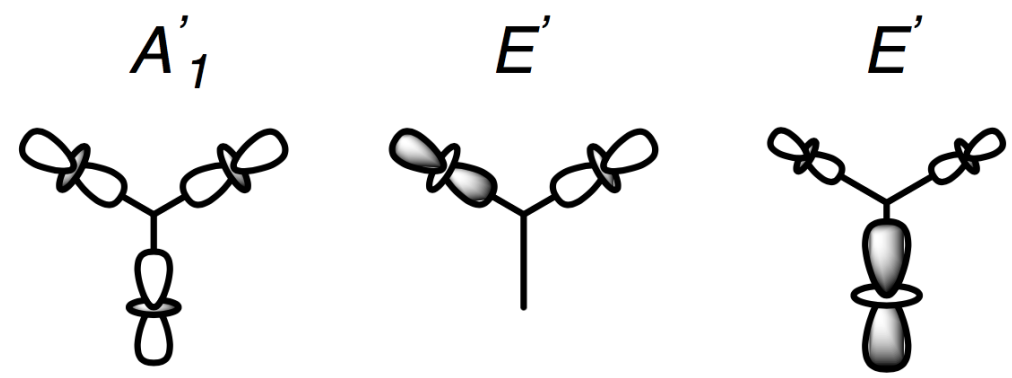

Fig. 5.5: Corresponding Ti- $d$ orbitals representing the local symmetry at an O-site (defect center), in the (110) plane. The $\mathrm{Ti} d$ states pointing towards the defect center are dominated by the approximate $\mathrm{D}_{3 \mathrm{~h}}$ symmetry of the defect. They are classified as $A_{1}^{\prime}$ and $E^{\prime}$. $E^{\prime}$ appears in two degenerated modifications. For the other $d$-orbitals on the Ti-neighbors of the defect, we refer to the approximate octahedral symmetry.

In order to quantify the defect structure we will refer to the triangle center. Because the actual symmetry of the defect is lower than $D_{3 \mathrm{~h}}$ we need an unambiguous definition 
of the defect center. We define the defect center as the point in the plane that has equal distance from all three adjacent Ti ions.

\subsubsection{Method}

The augmentation has been constructed as using the systematic projector construction [67]. For the augmentation, a $s^{2} p^{2} d^{1}$ set of projector functions was used for the Ti atom, and a $s^{2} p^{1} d^{1}$ set for $\mathrm{O}$ atom The superscripts denote the number of projector functions angular momentum channel. For the Ti atom, the $3 p$ core electrons were treated like the valence electrons, while the $3 s$ electrons were treated as core states.

A plane wave cutoff of 40 Ry has been chosen for the wave functions and 80 Ry for the charge density. The Brillouin-zone integration has been performed with the linear tetrahedron method [213, 214] and the so-called Blöchl corrections [215]. A $5 \times 5 \times 8 \mathrm{k}$-point mesh has been used for the unit cell containing two formula units. Defect calculations were simulated by $2 \times 2 \times 3$ supercells with 72 atoms plus the defect. All atomic positions are optimized without symmetry constraints. The theoretical lattice constant of the bulk has been obtained with the experimental[216] $c / a$ ratio of 0.644 . This lattice constant has been used for the supercells. Collinear spin polarization was allowed in all calculations.

Three different defect types are calculated: the oxygen vacancy $\mathrm{V}_{\mathrm{O}}$, the interstital hydrogen $\mathrm{H}_{\mathrm{i}}$ and the oxygen vacancy filled with hydrogen $\mathrm{H}_{\mathrm{O}}$. The interstital titanium $\mathrm{Ti}_{\mathrm{i}}$ was not considered as the concentration was found experimentally not to depend on the hydrogen partial pressure (Appendix. C). For every defect, the relevant charge states are considered, namely $\mathrm{V}_{\mathrm{O}}^{2+}, \mathrm{V}_{\mathrm{O}}^{+}, \mathrm{V}_{\mathrm{O}}^{0}, \mathrm{H}_{\mathrm{i}}^{+}, \mathrm{H}_{\mathrm{i}}^{0}, \mathrm{H}_{\mathrm{i}}^{-}, \mathrm{H}_{\mathrm{O}}^{+}, \mathrm{H}_{\mathrm{O}}^{0}$, and $\mathrm{H}_{\mathrm{O}}^{-}$.

The defect formation energy

$$
\Delta E_{f}[X]=E\left[\mathrm{TiO}_{2}: X\right]-E\left[\mathrm{TiO}_{2}\right]-\sum_{y \in\{e, \mathrm{H}, \mathrm{O}\}} \mu_{y} \eta_{y, X}
$$

allows to compare the thermodynamic stability of defects. For a defect $X$, the defect formation energy is calculated as difference of the supercell energy $E\left[\mathrm{TiO}_{2}: X\right]$ containing the defect $X$ and the supercell energy $E\left[\mathrm{TiO}_{2}\right]$ of the perfect crystal. Each energy is calculated with a relaxed atomic structure. The number of particles added during the formation of defect $X$ is denoted as $\eta_{e, X}$ for electrons, $\eta_{\mathrm{O}, X}$ for oxygen and $\eta_{\mathrm{H}, X}$ for hydrogen. These particles are taken from the respective particle reservoirs characterized by the corresponding chemical potentials, $\mu_{e}$ for electrons, $\mu_{\mathrm{O}}$ for oxygen atoms and $\mu_{\mathrm{H}}$ for hydrogen atoms.

We use the term chemical potential in the thermodynamic sense as the conjugate variable of the particle number respectively its density. The chemical potential of a particle type is therefore spatially constant for a system in thermal equilibrium. The chemical potential is often referred to as the electrochemical potential.

The thermodynamic charge state level

$$
\epsilon\left(q_{1} / q_{2}\right)=\frac{E\left[X^{q_{2}}\right]-E\left[X^{q_{1}}\right]}{q_{2}-q_{1}}
$$


is the position of the electron chemical potential for which two charge states $\left(q_{1}\right.$ and $\left.q_{2}\right)$ coexist. For lower Fermi levels, the more positive charge state is thermodynamically stable and the more negative charge state for higher Fermi levels.

\subsubsection{Interstitial hydrogen}

The positive interstitial hydrogen binds to an oxygen site as shown in Fig. 5.6a. The structural parameters are summarized in Tab. 5.3. The formation of an $\mathrm{OH}$ bond for the hydrogen interstitial has been reported also for anatase [174, 175, 177]. The $\mathrm{OH}$ bond is approximately perpendicular to the plane of the three $\mathrm{O}-\mathrm{Ti}$ bonds. The $\mathrm{OH}$ bond length is $1.003 \AA$, about $0.1 \%$ smaller than an $\mathrm{OH}$ bond in water [217]. The three O-Ti bond length increase by about $7.2 \%$ to $7.7 \%$, resulting in a trigonal pyramidal distortion of the oxygen coordination sphere shown in Fig. 5.6a. The OH group forms a hydrogen bond with one of the oxygen ions on the opposite side of the cage. Compared to water, the hydrogen bond of the hydrogen interstitial is about $1.3 \%$ larger [217]. The off-axis orientation of the hydrogen bond tilts the $\mathrm{OH}$ bond by $9^{\circ}$ away from the plane normal of the $\mathrm{O}$-Ti bonds.

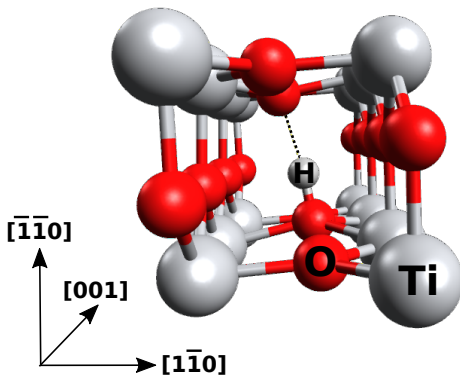

(a)

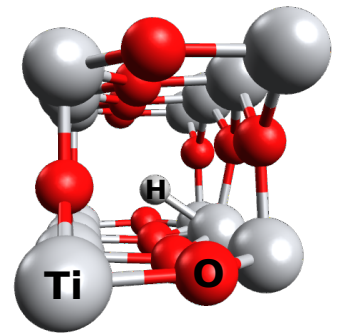

(b)

Fig. 5.6: (Color online) Structure of the (a) positive $\mathrm{H}_{\mathrm{i}}^{+}$and (b) negative $\mathrm{H}_{\mathrm{i}}^{-}$interstitial hydrogen in rutile $\mathrm{TiO}_{2}$. The neutral interstitial hydrogen is qualitatively similar to the positive one.

The neutral hydrogen interstitial assumes an atomic structure very similar to that of the positive hydrogen interstitial. Bond lengths change by less than $1.5 \%$. A small symmetry-lowering distortion can be traced to the electronic structure as discussed below.

Going to from the neutral to the negative charge state radically changes the bondnetwork. As shown in Fig. 5.6b, the negative hydrogen interstitial, binds to a cation, namely a Ti neighbor. The bond length is $1.84 \AA$. The Ti-H bond points towards one of the octahedral faces. As a result the adjacent $\mathrm{TiO}$ bonds bend away from the $\mathrm{Ti}-\mathrm{H}$ bond. The resulting $\mathrm{H}-\mathrm{Ti}-\mathrm{O}$ bond angle is $67^{\circ}$.

In the positive charge state of the interstitial hydrogen, we find an outward breathing of the three Ti-atoms by about $5.8 \%$ compared to the bulk, which shrinks with increasing 
Table 5.3: Structure parameters and energy level position of the defects. With $q$ we denote the net charge. The bond distances from the defect atoms $\mathrm{O}$ and $\mathrm{H}$ and the defect center $\mathrm{C}_{\mathrm{D}}$ to the neighboring Ti ions are given in $\AA$. The labelling of the Ti ions refers to that in fig. 5.9. The breathing distortion is expressed as distance of the neighboring Ti-ions from the defect center $\mathrm{C}_{\mathrm{D}}$ defined in computational details. The position of the defect levels in the optical band gap are expressed as their energetic separation $\Delta E$ in $\mathrm{eV}$ from the conduction-band edge.

\begin{tabular}{|c|c|c|c|c|c|c|c|c|c|c|c|}
\hline Compound & $\begin{array}{c}\text { Kröger- } \\
\text { Vink } \\
\text { symbol }\end{array}$ & $q_{\mathrm{j}}(\mathrm{e})$ & $\mathrm{O}-\mathrm{H}$ & $\begin{array}{c}\mathrm{Ti}_{1^{-}} \\
\mathrm{O}\end{array}$ & $\begin{array}{c}\mathrm{Ti}_{2^{-}} \\
\mathrm{O}\end{array}$ & $\begin{array}{c}\mathrm{Ti}_{3^{-}} \\
\mathrm{O}\end{array}$ & $\begin{array}{c}\mathrm{Ti}_{1^{-}} \\
\mathrm{H}\end{array}$ & $\begin{array}{c}\mathrm{Ti}_{2^{-}} \\
\mathrm{H}\end{array}$ & $\mathrm{Ti}_{3}-\mathrm{H}$ & $\mathrm{Ti}-\mathrm{C}_{\mathrm{D}}$ & $\begin{array}{c}\Delta E \\
(\mathrm{eV})\end{array}$ \\
\hline Undoped bulk & - & - & 0 & 1.947 & 1.947 & 1.948 & - & - & - & 1.961 & - \\
\hline \multirow[t]{3}{*}{ Interstitial $\mathrm{H}$} & $\mathrm{H}_{\mathrm{i}}^{+}$ & +1 & 1.003 & 2.098 & 2.109 & 2.110 & - & - & - & 2.074 & - \\
\hline & $\mathrm{H}_{\mathrm{i}}^{0}$ & 0 & 1.004 & 2.099 & 2.110 & 2.110 & - & - & - & 2.073 & - \\
\hline & $\mathrm{H}_{\mathrm{i}}^{-}$ & -1 & - & 1.935 & 1.977 & 1.977 & - & 1.842 & - & 1.957 & 1.62 \\
\hline \multirow[t]{3}{*}{ O vacancy } & $\mathrm{V}_{\mathrm{O}}^{2+}$ & +2 & - & - & - & - & - & - & - & 2.206 & - \\
\hline & $\mathrm{V}_{\mathrm{O}}^{+}$ & +1 & - & - & - & - & - & - & - & 2.210 & - \\
\hline & $\mathrm{V}_{\mathrm{O}}^{0}$ & 0 & - & - & - & - & - & - & - & 1.923 & 0.05 \\
\hline \multirow[t]{3}{*}{$\mathrm{O}$ vacancy containing $\mathrm{H}$} & $\mathrm{H}_{\mathrm{O}}^{+}$ & +1 & - & - & - & - & 1.998 & 1.999 & 2.034 & 2.012 & - \\
\hline & $\mathrm{H}_{\mathrm{O}}^{0}$ & 0 & - & - & - & - & 1.984 & 1.984 & 2.028 & 2.001 & - \\
\hline & $\mathrm{H}_{\mathrm{O}}^{-}$ & -1 & - & - & - & - & 1.976 & 1.976 & 2.021 & 1.994 & - \\
\hline
\end{tabular}

negative charge to about $5.7 \%$ for the neutral charge state to about $-0.2 \%$ inward breathing in the negative charge state. As shown in Tab. 5.3, the trend that the breathing increases when the defect becomes more positively charged, is common to all defect types considered. We define the breathing as the percentage increase of the distances of the Ti-neighbors from the triangle center defined earlier relative to the bulk.

The density of states for the hydrogen interstitial in the three charge states $\mathrm{H}_{i}^{+}, \mathrm{H}_{i}^{0}$ and $\mathrm{H}_{i}^{-}$is shown in Fig. 5.7. For each charge state, the total density of states of the supercell is shown in gray. The projected density of states onto the relevant orbitals are shown in the proximity of the defect. In particular, we distinguish the $d$-orbitals pointing towards the defect in $A_{1}^{\prime}$ and $E^{\prime}$ type as shown in Fig. 5.5.

For the positive and the neutral hydrogen interstitial the $\mathrm{OH} \sigma$-bond orbital splits off below the oxygen $2 p$ valence band. An iso-surface plot of the corresponding state is depicted in Fig. 5.7a and Fig. 5.7b. Clearly visible is also that the state extends along the hydrogen bond to the opposing $\mathrm{O}$ ion. There is no analogous state in the negatively charged hydrogen interstitial, because the $\mathrm{OH}$-bond has been replaced by a $\mathrm{Ti}-\mathrm{H}$ bond.

There are no gap states for the positive and neutral hydrogen interstitial. For the neutral hydrogen interstitial the Fermi level lays at the bottom part of the conduction band. As shown in the inset of Fig. $5.7 \mathrm{~b}$, the state is delocalized in the $t_{2 g}$-state on the Ti atom adjacent to the $\mathrm{OH}$ group as shown in the orbital representation. The defect state has $\pi^{*}$ (antibonding) character with respect to the TiO bonds. The antibonding character 


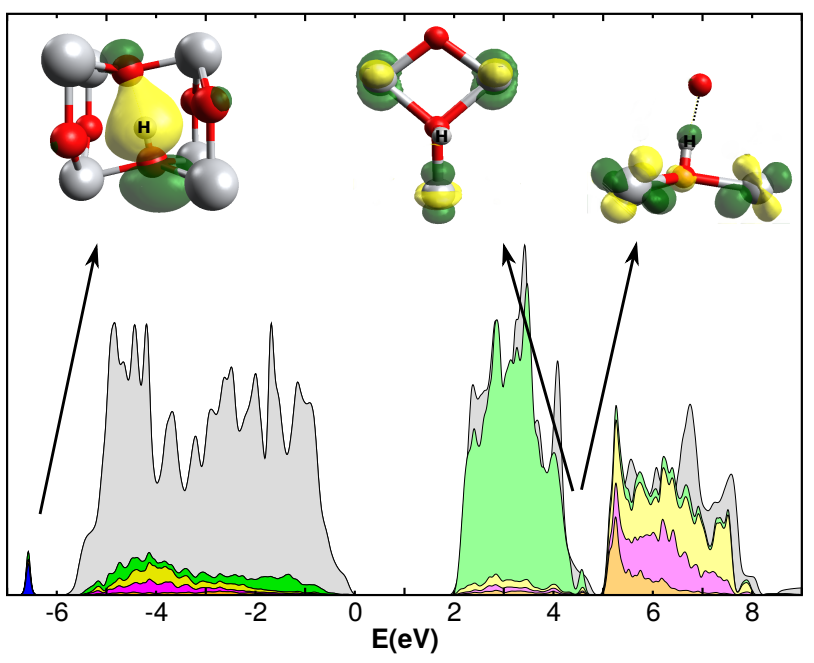

(a)

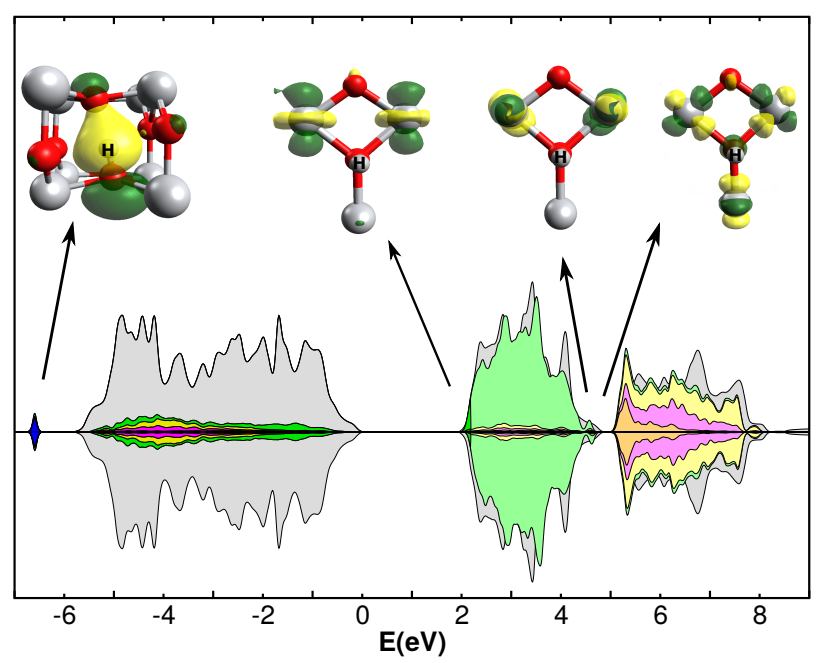

(b)

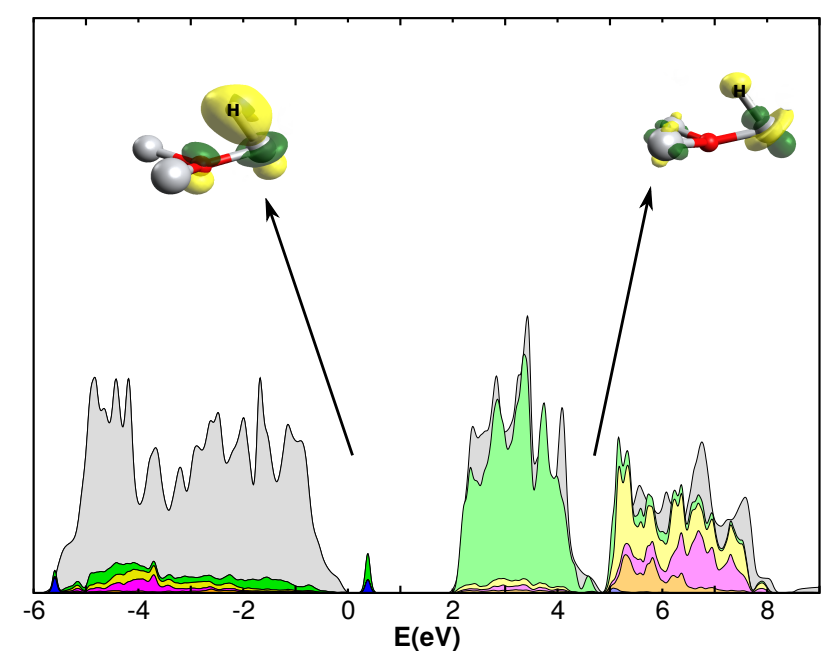

(c)

Fig. 5.7: DoS for the hydrogen interstitial in rutile $\mathrm{TiO}_{2}$. (a) $\mathrm{H}_{\mathrm{i}}^{+}$, (b) $\mathrm{H}_{\mathrm{i}}^{0}$ and (c) $\mathrm{H}_{\mathrm{i}}^{-}$for the positively, neutrally and negatively charged states, respectively, from top to bottom. Total density of state of bulk is represented with grey and hydrogen distribution with blue. The orbital representation of selected states are included, considering the respective sign of the wave function as indicated with the green $(+)$ and yellow $(-)$ color. The graphs for (a), (b), and (c) show the DoS for the $A_{1}^{\prime}$ (orange), $E^{\prime}$ (magenta), $\delta-e_{g}$ (yellow) and $t_{2 g}$-delta (green) orbitals on the three Ti neighbors of the defect. 
explains the expansion of the corresponding TiO-bonds by about 3.4-8.9\% (see. Tab. 5.3).

The neutral hydrogen interstitial may be considered as a pair of a positive hydrogen interstitial with an electron polaron on a Ti-site. The electron polaron requires the stabilization of the nearby positive defect: Without this stabilization the local ionic relaxation vanishes and defect level merges with the conduction band.

The negative hydrogen interstitial introduces a spin degenerate deep donor level in an approximate midgap position, that is $1.62 \mathrm{eV}$ below the conduction band edge. This state is a bond orbital between the hydrogen s-orbital and the $d$-orbital oriented along the Ti-H bond axis. There is an antibonding interaction with the adjacent oxygen ions consistent with their outward relaxation.

Of particular interest in relation to the measured EEL spectra are the defect induced changes of the Ti- $d$ states forming the conduction band.

For the positive hydrogen interstitial we observe that the $d$-density of states accumulates at the lower edge of the $t_{2 g}$ and the $e_{g}$ bands respectively. We attribute this to the electrostatic attraction of the electrons in the Ti-levels and the nearby positive defect. The opposite effect is seen in the density of states for the negative hydrogen interstitial where the $t_{2 g}$ states accumulate on the upper band edge and even form a split of state overlapping the continuum of $e_{g}$ states.

The Ti-H antibond appears in the quasi-gap between the $t_{2 g}$ and $e_{g}$ states at about $6 \mathrm{eV}$ as seen from the inset of fig 5.7.c. Also this antibond lifts some $t_{2 g}$ character above the $t_{2 g}$ continuum.

One common mechanism for the accumulation spectral weight between the $t_{2 g}$ and $e_{g}$ states is the Coulomb shift of the Ti- $d$ energy levels due to a nearby charged defect. If, on the one hand, the electrons on a $\mathrm{Ti}$ site experiences repulsive electric potential from a negative defect, the $t_{2 g}$ states of that site will accumulate at the upper edge of the $t_{2 g}$ band. If, on the other hand, the electrons on a $\mathrm{Ti}$ site experience an attractive electric potential from a positive defect, the $e_{g}$ states of that site will accumulate at the lower edge of the $e_{g}$ band. In both cases, additional spectral weight appears in the quasi gap between $t_{2 g}$ and $e_{g}$ states.

Interstitial hydrogen is thermodynamically stable only in the positive charge state as shown in Fig. 5.8. The neutral and negative charge states are unstable. The thermodynamic charge state level $\epsilon(+/ 0)$ lies $1.19 \mathrm{eV}$ above the conduction band minimum $\left(\epsilon_{C}\right)$ : for whole range of Fermi level below the hydrogen interstitial is positive. The charge state levels are summarized for the different defect species in Tab. 5.4.

In summary, the hydrogen in $\mathrm{H}_{\mathrm{i}}^{+}$and $\mathrm{H}_{\mathrm{i}}^{0}$, forms a bond with the oxygen atom, while $\mathrm{H}_{\mathrm{i}}^{-}$forms a bond with the $\mathrm{Ti}$ ion. No gap states are formed for the positive and neutral interstitial, while deep donor level in an approximate mid-gap position is present for the negative charge state. In the case of $\mathrm{H}_{\mathrm{i}}^{-}$, two electrons shared between a Ti-d orbital and hydrogen. In the context of the EELS, we observed two mechanism for accumulating spectral weight in between the $t_{2 g}$ and $e_{g}$ states. One is electrostatic in nature and the other one is related to a Ti-H antibond. Thermodynamically $\mathrm{H}_{\mathrm{i}}^{+}$is the stable defect species. 

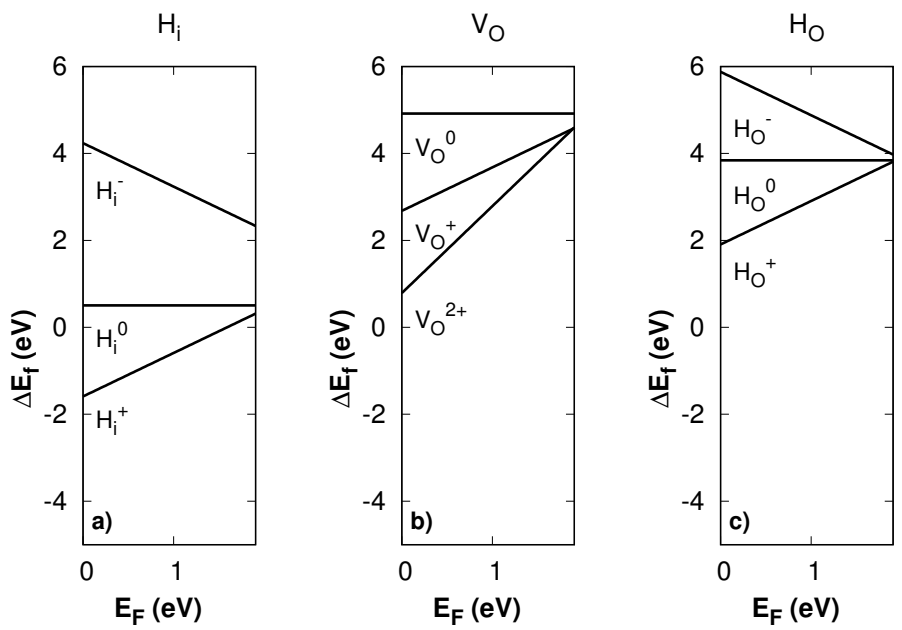

Fig. 5.8: Defect formation energies $\Delta E_{\mathrm{f}}$ as function of the Fermi level $E_{\mathrm{F}}$, for the three different defects a) $\mathrm{H}_{\mathrm{i}}$, b) $\mathrm{V}_{\mathrm{O}}$ and c) $\mathrm{H}_{\mathrm{O}}$ with respect to the different charge states (as marked in the graphs). All energies are given with respect to the valence band top $\left(\epsilon_{\mathrm{V}}\right)$ which is set to $0 \mathrm{eV}$.

Table 5.4: Charge state level $\epsilon$ for different defect species. $\epsilon$ is given relative to the conduction band minimum $\left(\epsilon_{\mathrm{C}}\right)$.

\begin{tabular}{ccc}
\hline \hline Defect & Charge states & $\epsilon(\mathrm{eV})$ \\
\hline $\mathrm{H}_{\mathrm{i}}$ & $(+/ 0)$ & $\epsilon_{\mathrm{C}}+0.191 \mathrm{eV}$ \\
$\mathrm{H}_{\mathrm{i}}$ & $(0 /-)$ & $\epsilon_{\mathrm{C}}+1.827 \mathrm{eV}$ \\
$\mathrm{H}_{\mathrm{i}}$ & $(+/-)$ & $\epsilon_{\mathrm{C}}+1.009 \mathrm{eV}$ \\
\hline $\mathrm{V}_{\mathrm{O}}$ & $(2+/+)$ & $\epsilon_{\mathrm{C}}-0.013 \mathrm{eV}$ \\
$\mathrm{V}_{\mathrm{O}}$ & $(+/ 0)$ & $\epsilon_{\mathrm{C}}+0.334 \mathrm{eV}$ \\
$\mathrm{V}_{\mathrm{O}}$ & $(2+/ 0)$ & $\epsilon_{\mathrm{C}}+0.160 \mathrm{eV}$ \\
\hline $\mathrm{H}_{\mathrm{O}}$ & $(+/ 0)$ & $\epsilon_{\mathrm{C}}+0.032 \mathrm{eV}$ \\
$\mathrm{H}_{\mathrm{O}}$ & $(0 /-)$ & $\epsilon_{\mathrm{C}}+0.131 \mathrm{eV}$ \\
$\mathrm{H}_{\mathrm{O}}$ & $(+/-)$ & $\epsilon_{\mathrm{C}}+0.081 \mathrm{eV}$ \\
\hline \hline
\end{tabular}

\subsubsection{Oxygen vacancy}

In $\mathrm{TiO}_{2}$, each $\mathrm{O}$ atom has an approximate trigonal coordination of three $\mathrm{Ti}$ atoms in a $\{110\}$ plane. The formation of an oxygen vacancy leaves three Ti ions undercoordinated as shown in Fig. 5.9. The relevant structural parameters are given in Tab. 5.3.

For the double positively charged oxygen vacancy $\mathrm{V}_{\mathrm{O}}^{2+}$, the adjacent $\mathrm{Ti}$ atoms relax outward by about $12.5 \%$ relative to the center as compared with the bulk. The outward relaxation shrinks to about $-1.9 \%$ for the singly positive oxygen vacancy. The neutral 


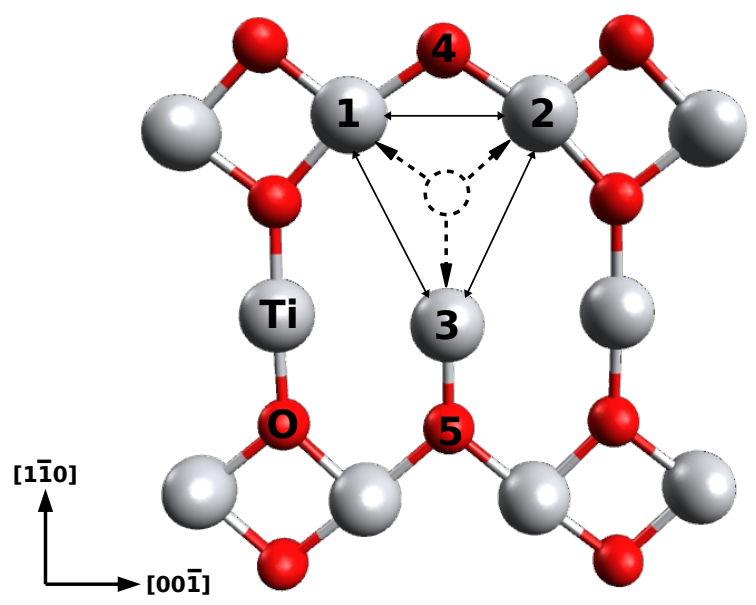

Fig. 5.9: (Color online) Structure of the oxygen-vacancy $\left(\mathrm{V}_{\mathrm{O}}\right)$ in rutile $\mathrm{TiO}_{2}$.

oxygen vacancy exhibits a inward breathing of $-3 \%$. The breathing seems to follow a simple electrostatic argument: The positive Ti-ions repel, and this repulsion is compensated by adding electrons to the defect. As electrons are added this outward relaxation is reduced and turned into a inward relaxation.

The DoS for the supercell with the oxygen vacancy in the three charge states $\mathrm{V}_{\mathrm{O}}^{2+}, \mathrm{V}_{\mathrm{O}}^{+}$ and $\mathrm{V}_{\mathrm{O}}^{0}$ is shown in Fig. 5.10.

As shown in Fig. 5.10a, the double positive oxygen vacancy $\mathrm{V}_{\mathrm{O}}^{2+}$ produces no deep levels in the band gap.

The Ti- $d$ states pointing into the vacancy with $A_{1}^{\prime}$ character are located at the bottom of or slightly below the conduction band. The corresponding two states with $E^{\prime}$ character are nearly degenerate and form two sharp peaks in the quasi gap between the Ti $t_{2 g}$ and $e_{g}$ states.

The Ti- $d$ states pointing into the vacancy, the $A_{1}^{\prime}$ and $E^{\prime}$ states, loose an antibond with the former oxygen neighbor. This antibond was responsible for the crystal field splitting that shifted these orbitals up, above the $t_{2 g}$ orbitals. In the absence of the oxygen ion, the $A_{1}^{\prime}$ and $E^{\prime}$ states are shifted down in energy, so that their energy average lies in the range of $t_{2 g}$ bands.

As we form the singly positive oxygen vacancy $\left(\mathrm{V}_{\mathrm{O}}^{+}\right)$, the additional electron enters the $t_{2 g}$. As shown in Fig. 5.10b this state lays in the conduction band edge in the the bottom part. The $A_{1}^{\prime}$ and $E^{\prime}$ states experiences a spin splitting.

In the neutral oxygen vacancy $\left(\mathrm{V}_{\mathrm{O}}^{0}\right)$ the defect level is filled with two electrons. As shown in Fig. 5.10c, it is located about $0.05 \mathrm{eV}$ below the conduction band edge.

The $d$-states with $E^{\prime}$ symmetry remain in the quasi gap between $e_{g}$ and $t_{2 g}$ states for all charge states and form sharp peaks in the DoS. 


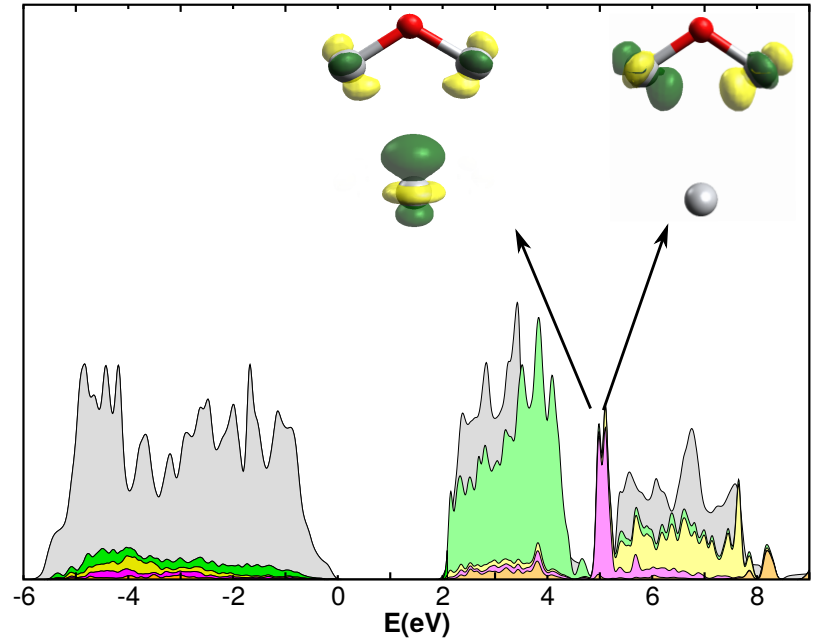

(a)

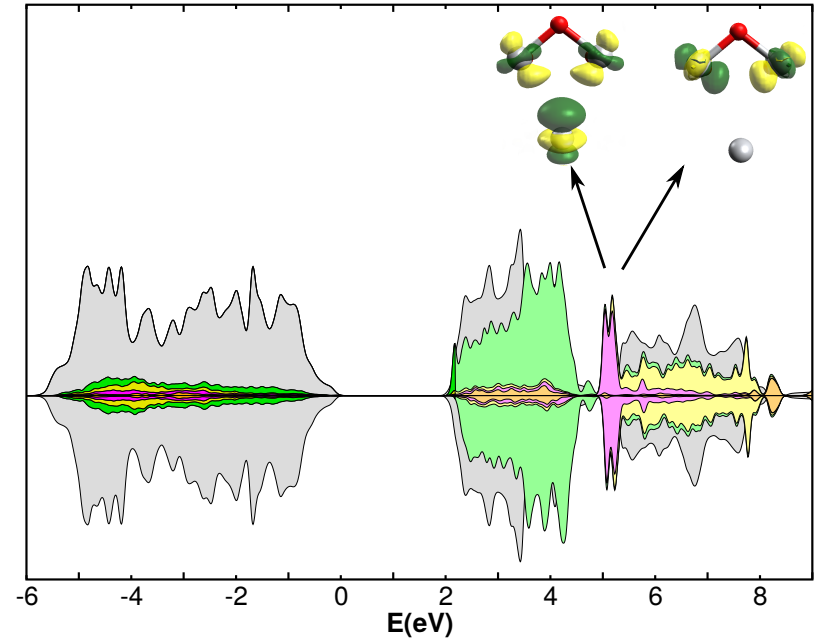

(b)

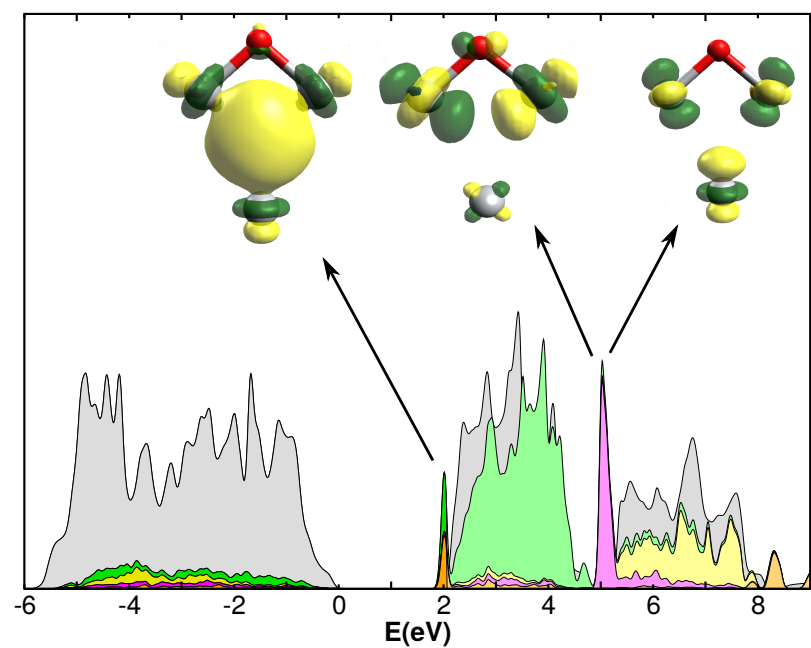

(c)

Fig. 5.10: DoS for an O-vacancy containing rutile, for the (a) $\mathrm{V}_{\mathrm{O}}^{2+}$, (b) $\mathrm{V}_{\mathrm{O}}^{+}$, and (c) $\mathrm{V}_{\mathrm{O}}^{0}$ denoting the doubly, the singly, and the neutrally charged states, respectively. Colors are represented based on Fig. 5.3 and 5.7. 
For the oxygen vacancy the doubly positive and the neutral charge state are thermodynamically stable as shown in Fig. 5.8. But considerable amount of the singly positive charge state are present as well. The corresponding charge state level $\epsilon(2+/ 0)$ lies $0.013 \mathrm{eV}$ below the conduction band minimum (see Tab. 5.4). Thus, if the oxygen vacancy is the dominant defect, the Fermi level will be pinned few tenths below the conduction band edge. This is sufficient to ionize about one in thousand of the oxygen vacancies at room temperature, which explains the color transition to deep blue of oxygen deficient rutile [218].

In summary, the oxygen vacancy exhibits sharp peaks in the DoS in the quasi gap between the $e_{g}$ and $t_{2 g}$ bands of rutile. They originate from $d$-states of the three Ti neighbors pointing into the oxygen vacancy with $E^{\prime}$ character with respect to the approximate $D_{3 \mathrm{~h}}$ symmetry of the oxygen vacancy. The corresponding orbital with $A_{1}^{\prime}$ character is located at or below the conduction band edge and contribute a deep donor levels about $0.05 \mathrm{eV}$ below the conduction band edge for the neutral charge state. The charging level is located $0.013 \mathrm{eV}$ above the conduction band minimum. The oxygen vacancy exhibits an outward breathing relaxation which shrinks and even turns into an inward breathing with increasing number of electrons.

\subsubsection{Hydrogen oxygen-vacancy complex}

After describing the isolated defects, the interstitial hydrogen $\mathrm{H}_{\mathrm{i}}$ and the oxygen vacancy $\mathrm{V}_{\mathrm{O}}$, we investigate here the pair of these two defects, namely the substitutional hydrogen on an oxygen site, $\mathrm{H}_{\mathrm{O}}$. As shown in Fig. 5.11, the hydrogen atom of $\mathrm{H}_{\mathrm{O}}$ is located almost at the defect center, respectively the position of the missing oxygen ion. The relevant distances for the charge states $\mathrm{H}_{\mathrm{O}}^{+}, \mathrm{H}_{\mathrm{O}}^{0}$ and $\mathrm{H}_{\mathrm{O}}^{-}$are summarized in Tab. 5.3.

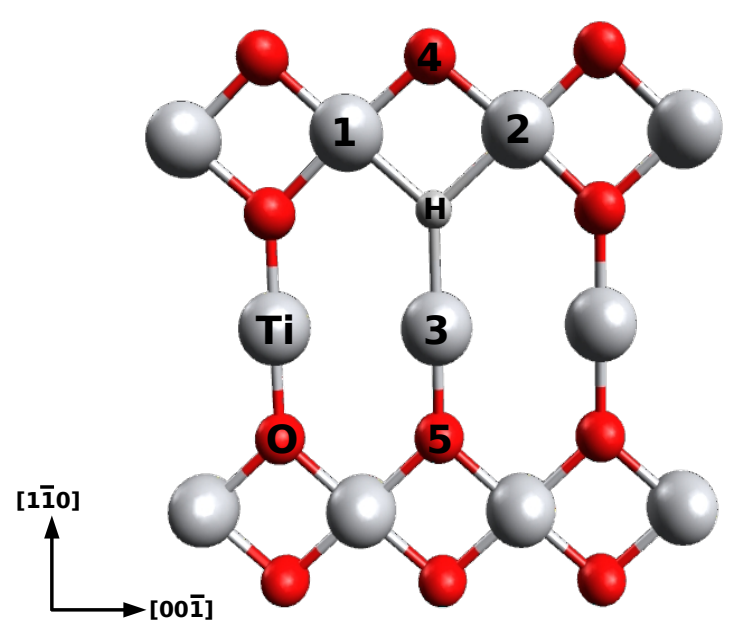

Fig. 5.11: (Color online) Structure of the hydrogen-complex $\mathrm{H}_{O}$ with an oxygen vacancy.

Like the other defects the outward breathing shrinks as electrons are added to the defect 
leading to a small inward relaxation for the most negative defect. The distances of the $\mathrm{Ti}$ ions from the defect center are $+2.6 \%$ for the positive, $+2.1 \%$ for the neutral and $+1.7 \%$ for the negative charge state compared to the bulk.

The neutral defect does not break the mirror symmetry, which relates two adjacent Ti ions (1 and 2 in Fig.5.11) to each other. Below we will attribute this effect to a delocalization of an electron. The symmetry is also again stored in the negative defect.

The corresponding DoS for the different charge states is shown in Fig. 5.12.

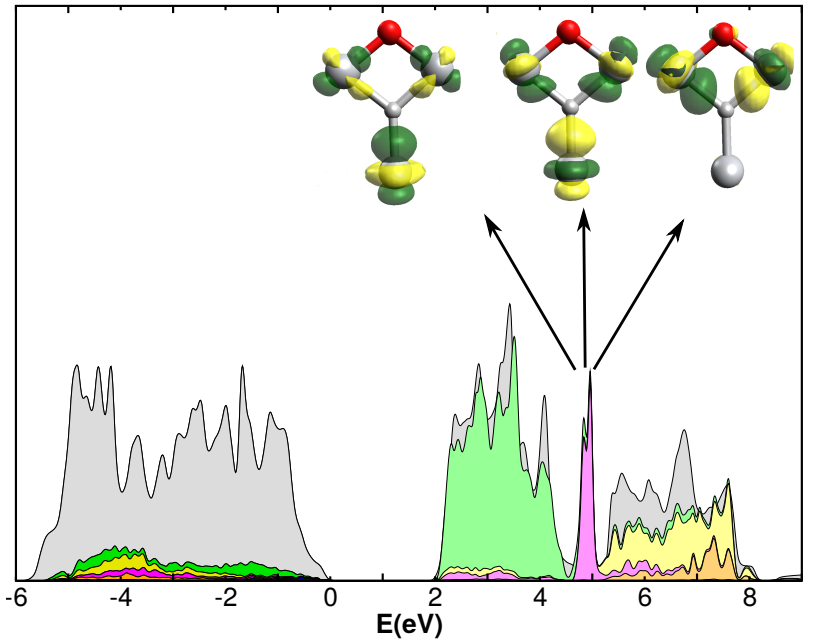

(a)

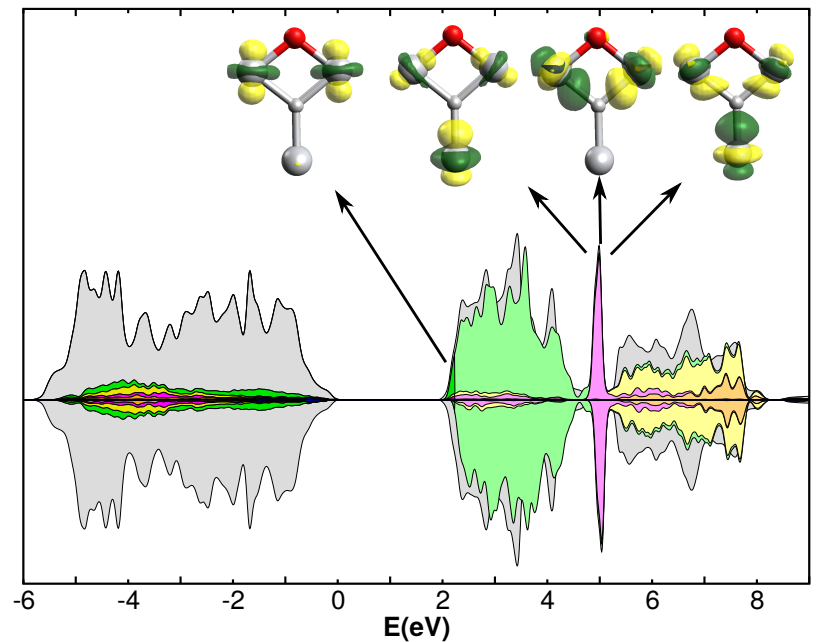

(b)

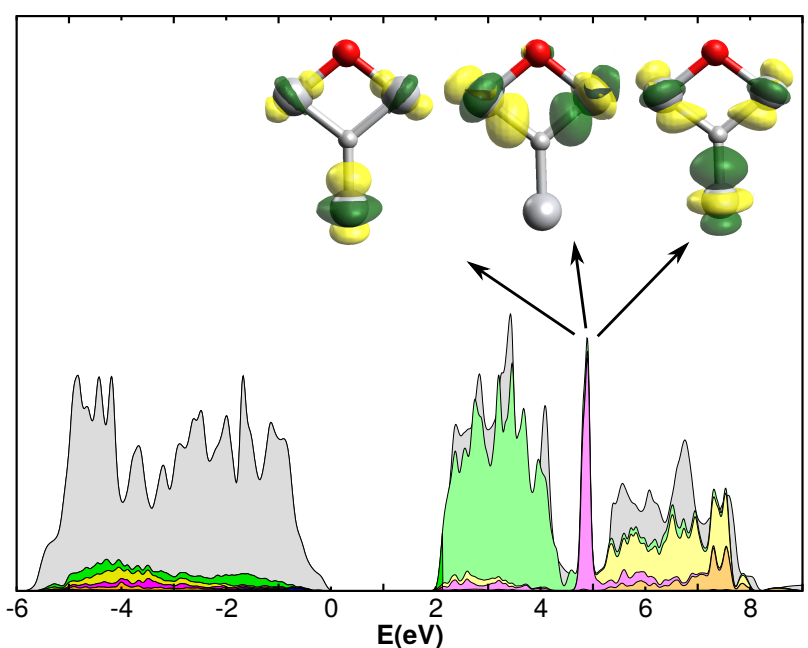

(c)

Fig. 5.12: DoS for the O-vacancy and hydrogen containing $\mathrm{TiO}_{2}$, (a) $\mathrm{H}_{\mathrm{O}}^{+}$, (b) $\mathrm{H}_{\mathrm{O}}^{0}$, and (c) $\mathrm{H}_{\mathrm{O}}^{-}$for the singly, neutrally and negatively charged states, respectively. Colors are represented based on Fig. 5.3 and 5.7.

The positive substitutional hydrogen $\mathrm{H}_{\mathrm{O}}^{+}$is obtained by inserting a proton into the 
neutral oxygen vacancy $\mathrm{V}_{\mathrm{O}}^{0}$. In comparison to the neutral vacancy, the dominant effect of the proton is to shift the defect level of $A_{1}^{\prime}$ character of the oxygen vacancy from the band gap downward into the continuum of the valence band. Therefore, the positive substitutional $\mathrm{H}_{\mathrm{O}}^{+}$does not have states in the band gap.

The Ti- $d$-states with $E^{\prime}$ symmetry with respect to the $\mathrm{D}_{3 \mathrm{~h}}$ symmetry group remain in the same position as in the oxygen vacancy, that is in the quasi gap between $t_{2 g}$ and $e_{g}$ states.

Addition of an electron to $\mathrm{H}_{\mathrm{O}}^{+}$leads to the neutral substitutional hydrogen $\mathrm{H}_{\mathrm{O}}^{0}$. The additional electron produces no deep level below the conduction band edge and delocalized in the conduction band. The occupied state has a $t_{2 g}$ nature and involves two of the three Ti neighbors of the substitutional hydrogen. Thus the symmetry is not broken, which favors two Ti ions. We attribute this to the electron delocalization due to the exchange term. The orbital is of $\pi$ character along the $\mathrm{Ti}-\mathrm{H}$ bond and has therefore no contribution from the central hydrogen atom. The deep level of the neutral substitutional hydrogen is at a similar energetic position as the one in the positive oxygen vacancy. However, it is of completely different nature. Rather it has similarity to the neutral interstitial hydrogen, where the deep level is due to a $t_{2 g}$ orbital. The deep level of the neutral interstitial hydrogen is due to an electron in a $t_{2 g}$ orbital localized in one of the three Ti neighbors of the central $\mathrm{OH}^{-}$group. The electron is electrostatically stabilized by the more positive charge of the $\mathrm{OH}^{-}$ion as compared to the $\mathrm{O}^{2-}$ ion in the bulk oxide. In $\mathrm{H}_{\mathrm{O}}^{0}$, the $\mathrm{OH}^{-}$ion is replaced by a $\mathrm{H}^{-}$ion. The additional electron enters into a $t_{2 g}$ orbital of the conduction band and experiences a similar electrostatic stabilization as the interstitial hydrogen.

Adding a further electron to form the negative charge state of the substitutional hydrogen $\mathrm{H}_{\mathrm{O}}^{-}$creates no deep level in the band gap of rutile. The electron is, however, spin paired, but the two electrons are delocalized on different Ti ions. This restores the original mirror symmetry of the defect. While our calculation allows for spin polarization, the total spin of this defect is set to zero. We anticipate that the triplet state is nearly degenerate to the singlet state.

Hydrogen in an oxygen vacancy $\mathrm{H}_{\mathrm{O}}$ is thermodynamically stable in positive charge state as shown in Fig. 5.8. Other charge-state levels are above the conduction band minimum.

While the oxygen vacancy tends to shift the Fermi level close to the conduction band, hydrogen tends to remove thin pinning of the Fermi level. Thus, hydrogen loading is expected to reduce the conductivity of oxygen deficient rutile.

In summary, the nature of the substitutional hydrogen can be described as a $\mathrm{H}^{-}$ion which replaces the $\mathrm{O}^{2-}$ ion of bulk rutile or the $\mathrm{OH}^{-}$ion of interstitial hydrogen. The electric and thermodynamic properties of substitutional and interstitial hydrogen are surprisingly similar. The electron state responsible for the charging process is in both cases a $d$-state on two Ti ions. Unlike interstitial hydrogen, the substitutional hydrogen does not change its site. It stays at the oxygen site for all three charge states. 


\subsection{Corrosion}

So far the calculations provide an explanation for the interfacial EELS profile. It is due to the band bending, which increases the concentration of hydrogenated oxygen vacancies at the interface. The hydrogenated oxygen vacancy, in turn, shifts two $e_{g}$ orbitals from the $e_{g}$ band into the quasi-gap between $t_{2 g}$ and $e_{g}$ orbitals, which appears like a downward shift.

However, so far there is no explanation for the sensitivity of the EELS on the hydrogen partial pressure. Here, we consider an explanation due to a hydrogen-induced corrosion of the oxide at the interface. The corrosion reaction envisaged is the formation of water and the simultaneous creation of (hydrogenated) oxygen vacancies due to the reaction with hydrogen.

$$
\frac{3}{2} \mathrm{H}_{2} \leftrightarrow \mathrm{H}_{\mathrm{O}}^{+}+\mathrm{H}_{2} \mathrm{O}+e^{-}
$$

Let me estimate the reaction energy (see Appendix. D), using $\mu_{e}=\epsilon_{c}+v_{e}\left(\vec{r}_{I}\right)-\Phi_{B n}$, where $\Phi_{B n}$ is Schottky barrier, $v_{e}\left(\vec{r}_{I}\right)$ shows the potential at the interface and $\epsilon_{c}$ denotes conduction-band minimum.

$$
\begin{aligned}
& \Delta G=G\left[\mathrm{H}_{2} \mathrm{O}\right]-\frac{3}{2} G\left[\mathrm{H}_{2}\right]+E\left[\mathrm{H}_{\mathrm{O}}^{+}\right]-v_{e}\left(\vec{r}_{I}\right)+\mu_{e} \\
& =G\left[\mathrm{H}_{2} \mathrm{O}\right]-\frac{3}{2} G\left[\mathrm{H}_{2}\right]+E\left[\mathrm{H}_{\mathrm{O}}^{+}\right]+\epsilon_{c}-\Phi_{B n} \\
& =\left(E\left[\mathrm{H}_{2} \mathrm{O}\right]+k_{\mathrm{B}} T \ln \left(\frac{\lambda_{T}^{3} p\left[\mathrm{H}_{2} \mathrm{O}\right]}{k_{\mathrm{B}} T}\right)+\ldots\right) \\
& -\frac{3}{2}\left(E\left[\mathrm{H}_{2}\right]+k_{\mathrm{B}} T \ln \left(\frac{\lambda_{T}^{3} p\left[\mathrm{H}_{2}\right]}{k_{\mathrm{B}} T}\right)+\ldots\right) \\
& +E\left[\mathrm{H}_{\mathrm{O}}^{+}\right]+\epsilon_{c}-\Phi_{B n} \\
& =\underbrace{E\left[\mathrm{H}_{2} \mathrm{O}\right]-E\left[\mathrm{H}_{2}\right]-\frac{1}{2} E\left[\mathrm{O}_{2}\right]}_{-2.55 \mathrm{eV}}-\frac{3}{2}(\underbrace{E\left[\mathrm{H}_{2}\right]-E\left[\mathrm{H}_{2}\right]}_{0}) \\
& +\underbrace{\underbrace{E\left[\mathrm{H}_{\mathrm{O}}^{+}\right]+\frac{1}{2} E\left[\mathrm{O}_{2}\right]-\frac{1}{2} E\left[\mathrm{H}_{2}\right]+\epsilon_{v}}_{1.906 \mathrm{eV}}+\underbrace{\epsilon_{c}-\epsilon_{v}}_{1.905 \mathrm{eV}}-\underbrace{\Phi_{B n}}_{0.788 \mathrm{eV}}}_{3.023 \mathrm{eV}} \\
& +\underbrace{\left(k_{\mathrm{B}} T \ln \left(\frac{\lambda_{T}^{3}\left[\mathrm{H}_{2} \mathrm{O}\right] \mathrm{Pa}}{k_{\mathrm{B}} T}\right)-\frac{3}{2} k_{\mathrm{B}} T \ln \left(\frac{\lambda_{T}^{3}\left[\mathrm{H}_{2}\right] \mathrm{Pa}}{k_{\mathrm{B}} T}\right)\right.}_{+0.212 \mathrm{eV}} \\
& +k_{\mathrm{B}} T \ln \left(\frac{p\left[\mathrm{H}_{2} \mathrm{O}\right] / \mathrm{Pa}}{\left(p\left[\mathrm{H}_{2}\right] / \mathrm{Pa}\right)^{3 / 2}}\right) \\
& +\Delta E_{v i b}+E_{\text {rot }}
\end{aligned}
$$

Based on this equation and using a hydrogen partial pressure of $10 \mathrm{~Pa}$, the reaction energy becomes negative, when the water partial pressure is below $\sim 7 \cdot 10^{-8} \mathrm{~Pa}$. Knowing 
that with our setup for the PBE functional reaction energies can be underestimated up to $\sim 0.3 \mathrm{eV}$ (see Tab. D.2), this pressure decreases to $\sim 6 \cdot 10^{-13} \mathrm{~Pa}$.

In the used ETEM, the background pressure is typically $\sim 10^{-4} \mathrm{~Pa}$. About $90 \%$ of that is water vapor. Using a cool finger can decrease the water partial pressure by roughly one order of magnitude. Hence, even with $\sim 10^{-5} \mathrm{~Pa}$ of water partial pressure the calculated lies a long way away to drive the corrosion reaction.

Hence, based on our calculated reaction energies and the needed corresponding water partial pressure, the corrosion reaction seems unlikely in our experiment conditions.

\subsection{Summary}

The $\mathrm{Ti} L_{3,2}$ EEL spectra in the vicinity of the $\mathrm{Pd} / \mathrm{r}-\mathrm{TiO}_{2}$ interface show changes in the $e_{g}$ states $\left(b / b^{\prime}\right.$ white line) in the hydrogen exposure of $10 \mathrm{~Pa}$. These observations are due to the point defects occurrence and their correlations. The calculated DoS show that all hydrogen induced defects $\left(\mathrm{H}_{\mathrm{i}}, \mathrm{V}_{\mathrm{O}}\right.$ and $\left.\mathrm{H}_{\mathrm{O}}\right)$ in all their charge states reveal a shift in the $e_{g}$ states. The calculated DoS of the defects can qualitatively explain the defect induced changes in rutile in the $2 \mathrm{~nm}$ vicinity of the $\mathrm{Pd} / \mathrm{r}-\mathrm{TiO}_{2}$ interface.

The thermodynamics calculations of the different defects show that only positive charge states $\left(\left(\mathrm{H}_{\mathrm{i}}^{+}, \mathrm{H}_{\mathrm{O}}^{+}, \mathrm{V}_{\mathrm{O}}^{2+}, \mathrm{V}_{\mathrm{O}}^{+}\right)\right)$are stable in the bulk rutile.

The multistate model [192] and the chosen experimental conditions of the system, suggests $\mathrm{H}_{\mathrm{O}}^{+}$and $\mathrm{H}_{\mathrm{i}}^{+}$to be the dominant defects in the vicinity of interface. Increasing the hydrogen partial pressure to $10 \mathrm{~Pa}$, only increases the interstitial hydrogen defect since no oxygen deficiency can occur. This is suggested by the multistate model. To create additional hydrogenated oxygen vacancies, a corrosion process is needed to form water. However, with the partial water pressure within the experimental condition, this process seems unlikely. Thus, the increasing of the interstitial hydrogen is more favorable and it is responsible for the state shifts observed in the EEL spectra. 


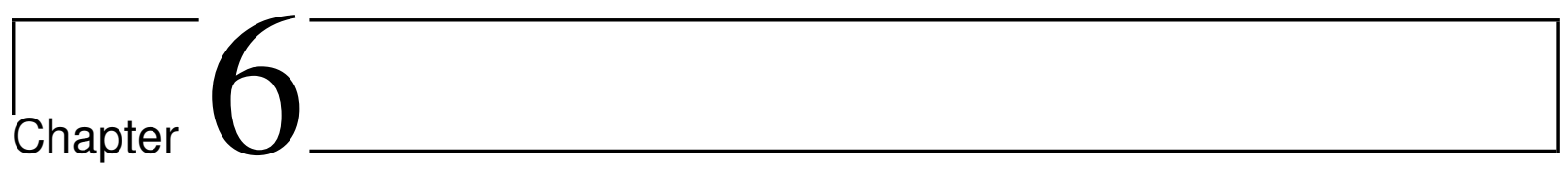

\section{Discussion}

Properties of manganites and water splitting on manganite surface have been studied. Our attention has focused mainly on the proper electronic description of the bulk, interface and oxygen evolution reactions. The partially filled $d$-orbital underlies the behavior of manganites. The octahedral environment of oxygen ions splits the $d$-orbitals of the $\mathrm{Mn}$ ions into a lower $t_{2 g}$ shell and a upper $e_{g}$ shell, the classic three-below-two crystal field splitting. Mn $d$-orbitals that point more toward the oxygen neighbors are higher in energy. In other words, the $e_{g}$ orbitals point directly towards the oxygen neighbors, while the $t_{2 g}$ orbitals point towards the octahedron faces. The $e_{g}$ orbitals form $\sigma$ antibonds with the oxygen neighbors, which lifts them energetically up relative to the $t_{2 g}$ orbitals which contribute only to weaker $\pi$ bonding. The corresponding bonding orbitals are located in the lower part of the oxygen valence band and are formally treated as oxygen orbitals.

At $T=0$ all states are filled up to the Fermi energy $\mathrm{E}_{F}$. The filled states are known as "electron states" and the empty states are known as "hole states". As a consequence of Hund's rule, the electron states split into spin-states with orientation parallel (spin-up) and antiparallel (spin-down). The spin states with the larger (smaller) electron population are called the majority (minority) states.

In $\mathrm{Pr}_{1-x} \mathrm{Ca}_{x} \mathrm{MnO}_{3}$, the three $t_{2 g}$ orbitals with majority state character are always occupied and they are energetically located deep in the oxygen valence band. The $e_{g}$ states with majority states character are located above the oxygen valence band. They are either empty, namely when $\mathrm{Mn}$ is in a $4+$ oxidation state such as $\mathrm{CaMnO}_{3}$, or filled with one electron in the $3+$ oxidation state such as $\mathrm{PrMnO}_{3}$.

The filling of the empty orbitals with electrons affect the local structure around the Mn ion. This is known as a Jahn-Teller effect. A Jahn-Teller ion is $\mathrm{Mn}^{3+}$. In the undoped system, such as $\mathrm{PrMnO}_{3}$, the $\mathrm{Mn}$ ion contains a single electron in the upper $e_{g}$ state. The $e_{g}$-orbitals are split into a lower filled and an upper unoccupied Jahn-Teller state. This splitting is caused by the octahedral distortion. In the case of a bond lengthening along the certain axis the antibonds of the orbital pointing to those oxygen neighbors weaken and thus drop in energy below the empty orbital. In contrast, for $\mathrm{Mn}^{4+}$ such as $\mathrm{CaMnO}_{3}$, 
the $e_{g}$ state with majority character is empty and no Jahn-Teller distortion occurs.

Hole doping of $\mathrm{PrMnO}_{3}$ introduces Zener polarons into the polaron crystal of cooperatively ordered Jahn-Teller polarons. A Jahn-Teller polaron is a $\mathrm{Mn}^{3+}$-site with one $e_{g}$ electron. Structurally, a Jahn-Teller polaron is characterized by a large Jahn-Teller distortion with $\sqrt{Q_{2}^{2}+Q_{3}^{2}}$. The Jahn-Teller splitting causes a band gap of $1.05 \mathrm{eV}$. Doping $\mathrm{PrMnO}_{3}$ with $\mathrm{Ca}$ introduces holes into the valence band of $\mathrm{PrMnO}_{3}$. In a rigid band structure picture, these holes result in a metallic behavior. However, such a state is energetically unfavorable. Instead, the system forms Zener polarons, where an electron is shared between two ferromagnetically aligned Mn-sites.

Characteristic for a prototypical Zener polaron is a Jahn-Teller distortion of two neighboring octahedra with an expansion towards the common bridging oxygen ion. The orbital picture is that of a lower, filled state formed by a symmetric combination of the lower JahnTeller orbital, i.e. a $d_{3 z^{2}-r^{2}}$ orbital pointing to the bridging oxygen. Due to symmetry, this orbital does not contain a contribution from the common bridging oxygen ion. Besides this "non-bonding" orbital, there is a empty antibonding orbital with opposite amplitudes for the two participating $d_{3 z^{2}-r^{2}}$ orbital. The bridging oxygen orbital mixes into this orbital and its antibonds are responsible for lifting this state above the "non-bonding" orbital. The admixture of an oxygen $p$-contribution to this unoccupied state is the reason attributing this state to an oxygen hole. The charge deficiency on the bridging oxygen $p$-orbital favors a larger bond angle of the oxygen bridge.

The Zener polaron opens up a band gap between filled and empty states of a half occupied pair of Mn sites. Our calculations indicate that the antibondig orbital is lifted above the energy of the Jahn-Teller partners, that is the corresponding $d_{x^{2}-y^{2}}$ orbitals.

This has important consequences on the optical absorption and the subsequent relaxation process. An optical absorption from one $e_{g}$ orbital to another on the same Mn site is strongly suppressed due to the dipole selection rule. The transition from the symmetric to the antisymmetric combination of the $d_{3 z^{2}-r^{2}}$ orbitals, however, is optically active. The oscillating dipole corresponds to the transfer of an electron from one Mn-site to the other. Below the excited state, however, there are unoccupied states with $d_{x^{2}-y^{2}}$ orbitals. The excited electron will relax into those orbitals. A recombination of the resulting electronhole pair via optical emission from these energy levels is strongly suppressed due to the different symmetry of the electron and hole states: In terms of an idealized straight oxygen bridge, the hole is in an axially symmetric-state, while the electron occupies the state with two mirror axes through the bond axis.

Thus, while the excitation of an electron-hole pair on a Zener polaron is highly efficient, the recombination process is strongly suppressed. This is one possible explanation of the long lifetimes of the excited electrons in manganites near the half doped regime.

In the CE structure, we find a magnetically collinear structure with ferromagnetically aligned zig-zag chains. The resulting electronic structure can be described as a ferromagnetic chain of Zener polarons. In our calculations, the dimerization into individual Zener polarons is lifted in favor of a symmetric state in between two alternative, energetically degenerate dimerization patterns. Despite of this, we will name this structure a ferromag- 
netic chain of Zener polatons, because the form the conceptual basis also for the symmetric structure.

The ferromagnetic chain of Zener polarons has two distinct Mn sites, one in the center and one at the corner of a zig-zag chain. This is noteworthy, because the atoms at the corner exhibit only a very weak Jahn-teller distortion, an observation that has been attributed to a charge order along the chain. The Jahn-Teller distortion disappears, however, not due to charge order, but because both $e_{g}$ states of the corner atoms are equally occupied.

Passing from $\mathrm{Pr}_{1-x} \mathrm{Ca}_{x} \mathrm{MnO}_{3}$ to $\mathrm{La}_{1-x} \mathrm{Sr}_{x} \mathrm{MnO}_{3}$, in the half doped system the orthorhombic structure is replaced by cubic structure. Instead of the Zener polarons and insulating behavior, the half metallic behavior is obtained. This is because of the larger hopping amplitude from $\mathrm{Mn}$-site to the nearest $\mathrm{Mn}$ ions. In the orthorhombic structure $\mathrm{Mn}-\mathrm{O}-\mathrm{Mn}$ angle is less than $180^{\circ}$ which causes less amount of hopping, while in the cubic structure this angle is close to $180^{\circ}$.

The gross feature of the electronic structure of manganites have been discussed earlier. Now, we will focus on oxygen evolution reactions on the manganite surfaces.

We have demonstrated that hemibonding interactions are a crucial parameter in the electro- or photo-catalysis of oxygen on manganite surfaces. The energy diagram rationalizes the presence of $\mathrm{OH}$ radical, $\mathrm{H}_{2} \mathrm{O}_{2}$, and $\mathrm{O}_{2}$ evolution. It shows that manganites are known to produce first $\mathrm{H}_{2} \mathrm{O}_{2}$, and then form $\mathrm{O}_{2}$ molecule. This proposed mechanism offers insight into the OER catalysts, and allows for the rationalization of the Fermi energy through transition metal $3 d$ and oxygen $2 p$ partial density of states at the surface. The role of oxygen vacancy defects cannot be ignored. It should be a critical component in the benchmarking of metal oxide oxygen electro- or photo-catalysts.

The acid-base mechanism was considered that shows the O-O bond is formed via a nucleophilic attack on the hydroxide species. By contrast, the direct-coupling mechanism which shows two metal oxo species coming close together, is not likely. The first hole localizes between hydroxide group and water molecule. It causes a rotation of water molecule to make hemibonding interaction. Then a proton releases from water and makes a hydronium on the top layer of water molecules. The second hole makes the O-O bond stronger and forms a peroxide species on the manganite surface. By releasing the next proton, peroxide species turn to the hydroperoxide species. Ultimately, the third and fourth holes are responsible for the $\mathrm{O}-\mathrm{O}$ bond formation and the displacement of $\mathrm{O}_{2}$ from the catalyst.

Our results predict that lattice oxygen atoms are not likely to involve in the OER on $\mathrm{CaMnO}_{3}$ (001). The precursor of molecular $\mathrm{O}_{2}$ is a surface hydroxide and the water molecule. Our result further suggest that $\mathrm{O}_{2}$ evolution pathway differs from the sequential pathway including metal oxo species. To understand the reason for this difference, we notice that on the $\mathrm{CaMnO}_{3}$ (001) surface ${ }^{*} \mathrm{O}$ adatoms at cations $\mathrm{Mn}$ sites are found to have instability and ${ }^{*} \mathrm{OH}$ can readily form via the nucleophilic attack of a water molecule. Our results thus suggest that the $\mathrm{O}$ source for the $\mathrm{O}_{2}$ molecule is largely determined by the geometry of * $\mathrm{OH}$ species, highlighting the important dependence of the OER pathway on the surface structure.

To gain more insight into the oxygen vacancy and presence of hydrogen inside the 
oxygen vacancy, hydrogen related defects in the rutile $\mathrm{TiO}_{2}$ was studied.

Our simulations give insights in the interaction of hydrogen with rutile in the vicinity of a palladium interface. With EELS we revealed a shift of Ti $d$ states in a $2 \mathrm{~nm}$ vicinity of the interface of about $1 \mathrm{eV}$ to $2 \mathrm{eV}$. Our simulations reveal that hydrogen related defects show shifts of states in a similar energy range. Therefore we explain the EELS findings with the presence of hydrogen related defects in the vicinity of the interface. Simulation of the defects thermodynamics reveal the presence of doubly charged and single positively charged oxygen vacancies, positively interstitial hydrogen and positively charge hydrogen in oxygen vacancies.

The Pd-related MIGS in the local vicinity of about $3 \AA$ to the interface are likely to result in high local oxygen vacancies densities. These vacancies easily trap hydrogen and relate to a Fermi level pinning at $0.01 \mathrm{eV}$ below the conduction band. Further hydrogen is solved in interstitial sites, giving rise to a Fermi level position being below the conduction band. This Fermi level only accounts for the bulk. At the interface, the Fermi level is pinned differently, maybe affected by the $\mathrm{H}_{\mathrm{O}}$ defects. The related band bending shifts the $\mathrm{H}_{i}$ defects state above the Fermi level thereby creating a positively charge region. The extension of this region can be in the order of $3 \mathrm{~nm}$, especially when the mobility of hydrogen is considered. The positive charge state of the interstitial hydrogen possesses different DoS compared to vacancy DoS. The observed shift of about $100 \mathrm{meV}$ can be explained, for high defect densities in the depletion layer. 


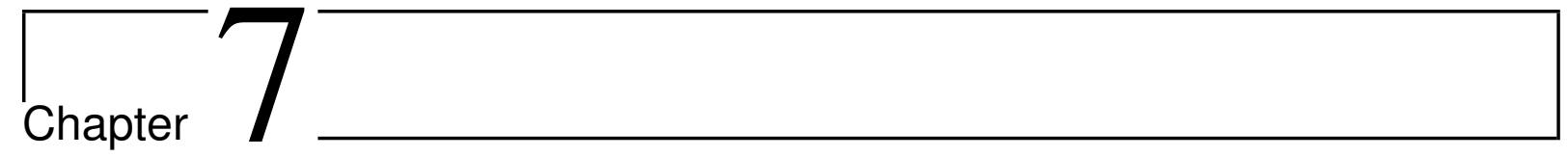

\section{Summary}

The electronic, magnetic and atomic structure of $\operatorname{Pr}_{1-x} \mathrm{Ca}_{x} \mathrm{MnO}_{3}$ have been investigated using combination of first-principles calculations, x-ray photoelectron spectroscopy (XPS), x-ray absorption spectroscopy (XAS), and electron-energy loss spectroscopy (EELS). With the compositions $x=0,1 / 2$, and $x=1$ and variety magnetic orders, we cover the entire doping range. Jahn-Teller as well as Zener polaron orders are considered. The comparison of experimental data with calculated spectra opened up new insight far beyond of the individual techniques.

We use local hybrid functionals in our calculations to account for the Coulomb interaction on the Mn-sites and to obtain the physically correct description of the electronic structure. With the local hybrid functional we avoid the well known deficiencies of densityfunctional calculations, which do not account explicitly for the strong Coulomb interaction in the Mn- $d$ shell. The free parameters of the local hybrid functionals have been determined by comparison with measured XPS spectra. The obtained admixture of the Fock-term is substantially smaller than the value suggested on the basis of perturbation theory [61]. For $\mathrm{Pr}_{1 / 2} \mathrm{Ca}_{1 / 2} \mathrm{MnO}_{3}$ we have explored two distinct magnetic orders, CE and E-type, with low energy, which exhibit a similar band gap. Together with a phase suggested by Giovanetti et al. [44], they indicate a pathway for the thermalization of the magnetic order.

With obtained hybrid factors, we investigated the electronic structure of $\mathrm{La}_{1-x} \mathrm{Sr}_{x} \mathrm{MnO}_{3}$ with first-principles calculations. With the compositions $x=0,1 / 3$, and $x=1$ the entire doping range have been covered. Jahn-Teller polaron as well as half-metallic system are considered. The comparison of $\mathrm{Pr}_{1-x} \mathrm{Ca}_{x} \mathrm{MnO}_{3}$ and $\mathrm{La}_{1-x} \mathrm{Sr}_{x} \mathrm{MnO}_{3}$ provides a detailed and deep understanding of manganites.

Passing from bulk to surface, a number of competing mechanisms for water splitting reactions are discussed. The role of correlated processes and fluctuations of the catalyst surface in contact with water are explored. Based on the dimeric water, we suggested a mechanism for oxygen evolution reaction on $\mathrm{CaMnO}_{3}$ (001) surface. The calculated DoS and orbital representations shed lights on the reactions steps. The energetic shows more stability for hemibonding interaction compared to the proton transfer. We demonstrated 
that lattice oxygen participation is unlikely for $\mathrm{CaMnO}_{3}(001)$.

To shed light on defects that play roles in the physical and chemical properties of metal oxides, a common native point defect, oxygen vacancy, and its combination with hydrogen in the well-known metal oxide $\mathrm{TiO}_{2}$ are studied. We demonstrate that in contact with palladium $(\mathrm{Pd})$ under exposure of hydrogen, the interstitial hydrogen and the hydrogenoxygen vacancy complex are actually positively charged and more stable. With these simulations, changes in EEL spectra collected experimentally in the vicinity of $\mathrm{Pd} / \mathrm{TiO}_{2}$ interface are explained. 
$\prod_{\text {Appendix }}$

\section{DFT}

\section{A.1 Derivation and formalism}

In this section I am following the lecture notes of P. Blöchl [219].

In order to obtain the ground state energy, $E, 2^{N}$ integrations in $3 N$ dimensions must be done, i.e.

$$
E=\langle\phi|H| \phi\rangle=\int d^{4} \mathbf{x}_{1} \cdots \int d^{4} \mathbf{x}_{N} \phi^{\star}\left(\mathbf{x}_{1}, \ldots, \mathbf{x}_{N}\right) H \phi\left(\mathbf{x}_{1}, \ldots, \mathbf{x}_{N}\right)
$$

All $N$-electron wave functions $\phi\left(\mathbf{x}_{\mathbf{1}}, \ldots, \mathbf{x}_{\mathbf{N}}\right)$ obey the Pauli principle, that means when two particles are exchanged, the sign of the wave function will change. In this notation, $\mathbf{x} \equiv(\mathbf{r}, s)$ which is combination of the electron position and the spin index. Similarly, the notation of a four-dimensional integral $\int d^{4} \mathbf{x}:=\sum_{s} \int d^{3} \mathbf{r}$ is used for the sum over spin indices and the integral over the position as well as, $\delta\left(\mathbf{x}-\mathbf{x}^{\prime}\right):=\delta_{s, s^{\prime}} \delta\left(\mathbf{r}-\mathbf{r}^{\prime}\right)$, which denotes the product of Kronecker delta of the spin coordinates and Dirac's delta function for the positions.

However, only two types of integrals appear in the expression of ground-state energy. To get more insight, number of definitions related to density matrices [220] are given here.

\section{N-particle density matrix}

Any density corresponding to an interacting $N$-electron density can be written as the matrix elements of the density operator. Thus

$$
\hat{\rho}^{(N)}=|\phi\rangle\langle\phi|
$$


which is the same density operator that has been used in statistical mechanics. The density matrix element can be described as

$$
\rho^{(N)}\left(\mathbf{x}_{1}, \ldots, \mathbf{x}_{N} ; \mathbf{x}_{1}^{\prime}, \ldots, \mathbf{x}_{N}^{\prime}\right)=\left\langle\mathbf{x}_{1}, \ldots, \mathbf{x}_{N}\left|\hat{\rho}^{(N)}\right| \mathbf{x}_{1}^{\prime}, \ldots, \mathbf{x}_{N}^{\prime}\right\rangle
$$

The $N$-particle density matrix contains all the information of the wavefunction because the expectation value of any operator can be determined from that. This can be obtained by writing,

$$
\begin{aligned}
\langle\phi|\hat{A}| \phi\rangle= & \int d^{4} \mathbf{x}_{1} \cdots \int d^{4} \mathbf{x}_{N} \int d^{4} \mathbf{x}_{1}^{\prime} \ldots \int d^{4} \mathbf{x}_{N}^{\prime} \\
& \rho^{(N)}\left(\mathbf{x}_{1}, \ldots, \mathbf{x}_{N} ; \mathbf{x}_{1}^{\prime}, \ldots, \mathbf{x}_{N}^{\prime}\right)\left\langle\mathbf{x}_{1}^{\prime}, \ldots, \mathbf{x}_{N}^{\prime}|\hat{A}| \mathbf{x}_{1}, \ldots, \mathbf{x}_{N}\right\rangle
\end{aligned}
$$

A stationary electronic state is then described by the expectation value of the Hamiltonian operator which can be calculated from the $N$-particle density matrix. However, special form of the Hamiltonian allows to use the simplest forms of density matrix that is called reduced density matrices. We define those in the following.

\section{One-particle and two-particle reduced density matrix}

A Hamiltonian of $N$-electron system can be described by three terms, as shown in Eq. 2.10. With those quantities the total energy is

$$
\begin{aligned}
E= & \langle\phi|H| \phi\rangle=\int d^{4} \mathbf{x}^{\prime} \int d^{4} \mathbf{x} \delta\left(\mathbf{x}^{\prime}-\mathbf{x}\right)\left(-\frac{\hbar^{2}}{2 m} \nabla^{2}+v_{e x t}(\mathbf{r})\right) \rho^{(1)}\left(\mathbf{x}, \mathbf{x}^{\prime}\right) \\
& +\frac{1}{2} \int d^{3} \mathbf{r} \int d^{3} \mathbf{r}^{\prime} \frac{e^{2} n^{(2)}\left(\mathbf{r}, \mathbf{r}^{\prime}\right)}{4 \pi \epsilon_{0}\left|\mathbf{r}-\mathbf{r}^{\prime}\right|}
\end{aligned}
$$

where $m$ denotes the electron mass, $\epsilon_{0}$ shows the vacuum permittivity, $e$ is the elementary charge and $\hbar$ is the Planck constant divided by $2 \pi$. The Coulomb potentials of the nuclei have been combined into an external potential $v_{e x t}(\mathbf{r}) . \rho^{(1)}\left(\mathbf{x}, \mathbf{x}^{\prime}\right)$ and $n^{(2)}\left(\mathbf{r}, \mathbf{r}^{\prime}\right)$ are the one-particle and two-particle reduced density matrix respectively. They can be calculated as

$$
\rho^{(1)}\left(\mathbf{x}, \mathbf{x}^{\prime}\right):=N \int d^{4} \mathbf{x}_{2} \cdots \int d^{4} \mathbf{x}_{N} \phi\left(\mathbf{x}, \mathbf{x}_{2}, \ldots, \mathbf{x}_{N}\right) \phi^{*}\left(\mathbf{x}^{\prime}, \mathbf{x}_{2}, \ldots, \mathbf{x}_{N}\right)
$$

and 


$$
n^{(2)}\left(\mathbf{r}, \mathbf{r}^{\prime}\right):=N(N-1) \sum_{s, s^{\prime}} \int d^{4} \mathbf{x}_{3} \cdots \int d^{4} \mathbf{x}_{N}\left|\phi\left(\mathbf{x}, \mathbf{x}^{\prime}, \mathbf{x}_{3}, \ldots, \mathbf{x}_{N}\right)\right|^{2}
$$

It should be noted that evaluations of all expectation values of one-particle operators such as the kinetic energy and the external potential energy can be calculated by oneparticle reduced density matrix, while the interaction between electrons can be determined by two-particle reduced density matrix.

\section{Electron density and exchange-correlation hole}

The electron density operator at a position $\mathbf{r}$ is

$$
\hat{n}(\mathbf{r})=\sum_{s}|\mathbf{r}, s\rangle\langle\mathbf{r}, s|
$$

thus, the electron density can be expressed by one particle density matrix as

$$
n\left(\mathbf{r}_{0}\right)=\sum_{s} \hat{\rho}^{(1)}\left(\mathbf{r}_{0}, s ; \mathbf{r}_{0}, s\right)=\sum_{s} \rho^{(1)}(\mathbf{x}, \mathbf{x})=\sum_{s} \sum_{n} \bar{f}_{n} \varphi_{n}^{*}(\mathbf{x}) \varphi_{n}(\mathbf{x})
$$

The eigenstates $\varphi_{n}(\mathbf{x})$ are called natural orbitals and the eigenvalues $\bar{f}_{n}$ are their occupations. The index $n$ labeling the natural orbitals may stand for a set of quantum numbers. With the natural orbitals the total energy obtains the form

$$
\begin{aligned}
E=\sum_{n} \bar{f}_{n} \int d^{4} \mathbf{x} \varphi_{n}^{*}(\mathbf{x}) \frac{-\hbar^{2}}{2 m} \nabla^{2} \varphi_{n}(\mathbf{x})+ & \int d^{3} \mathbf{r} v_{\text {ext }}(\mathbf{r}) n^{(1)}(\mathbf{r}) \\
& +\frac{1}{2} \int d^{3} \mathbf{r} \int d^{3} \mathbf{r}^{\prime} \frac{e^{2} n^{(2)}\left(\mathbf{r}, \mathbf{r}^{\prime}\right)}{4 \pi \epsilon_{0}\left|\mathbf{r}-\mathbf{r}^{\prime}\right|}
\end{aligned}
$$

In a classical description, which has uncorrelated electrons, the probability of finding the pair of electrons at the positions $\mathbf{r}$ and $\mathbf{r}^{\prime}$ is simply given by $n^{(2)}\left(\mathbf{r}, \mathbf{r}^{\prime}\right)=n^{(1)}(\mathbf{r}) n^{(1)}\left(\mathbf{r}^{\prime}\right)$. It shows the classical Coulomb repulsion, or Hartree energy. But in reality, electrons are correlated and they obey Fermi statistics. Moreover, electrons kept apart by the Pauli-exclusion principle. Therefore, these exchange and correlation interactions affects the electron density. The electron density at position $\mathbf{r}$ reduces compare to the classical value, because of the instantaneous position of the second electron located at $\mathbf{r}^{\prime}$. Hence, each electron creates a depletion, or let say hole, of electron density around itself. This is 
a direct consequence of exchange-correlation effects. The two-particle density can now be written by the density and the exchange-correlation hole function as,

$$
n^{(2)}\left(\mathbf{r}, \mathbf{r}^{\prime}\right)=n^{(1)}(\mathbf{r}) n^{(1)}\left(\mathbf{r}^{\prime}\right)+n^{(1)}(\mathbf{r}) h\left(\mathbf{r}, \mathbf{r}^{\prime}\right)
$$

where the quantum effects are in the exchange-correlation hole density, $h\left(\mathbf{r}, \mathbf{r}^{\prime}\right)$. It also affects the Coulomb repulsion between the electrons, by the attraction of the electron to its exchange-correlation hole. Coulomb repulsion is reduced relative to the Hartree energy by this effect. The exchange-correlation hole satisfies an important normalization condition known as a sum rule,

$$
\int d \mathbf{r}^{\prime} h\left(\mathbf{r}, \mathbf{r}^{\prime}\right)=-1
$$

This implies that an electron in an $N$-electron system, always sees $N-1$ other electrons. In other word, the exchange-correlation hole has a deficit of exactly one electron.

\section{Exact definition of exchange and correlation}

Now, the interaction energy can be described by the two-particle density. According to Eq. 2.30, the Coulomb interaction between electrons can be written by two terms. The first part is the so-called Hartree energy

$$
E_{H}=\frac{1}{2} \int d^{3} \mathbf{r} \int d^{3} \mathbf{r}^{\prime} \frac{e^{2} n^{(1)}(\mathbf{r}) n^{(1)}\left(\mathbf{r}^{\prime}\right)}{4 \pi \epsilon_{0}\left|\mathbf{r}-\mathbf{r}^{\prime}\right|}
$$

and the second part is the potential energy of exchange and correlation

$$
U_{x c}=\int d^{3} \mathbf{r} n^{(1)}(\mathbf{r}) \frac{1}{2} \int d^{3} \mathbf{r}^{\prime} \frac{e^{2} h\left(\mathbf{r}, \mathbf{r}^{\prime}\right)}{4 \pi \epsilon_{0}\left|\mathbf{r}-\mathbf{r}^{\prime}\right|}
$$

Note that $U_{x c}$ is not the exchange-correlation energy $\left(E_{x c}\right)$. The difference between these two quantities is a kinetic energy correction. In order to facilitate the calculation of the kinetic energy, the interacting electron system maps onto a non-interacting electrons with the same density as a true system $\left(T_{s}\left(n^{(1)}\right)\right)$. The difference relative to the real system is added to the potential energy of exchange and correlation. Thus, the exchange-correlation energy can be expressed as 


$$
E_{x c}=U_{x c}+\sum_{n} \bar{f}_{n} \int d^{4} \mathbf{x} \varphi_{n}^{*}(\mathbf{x}) \frac{-\hbar^{2}}{2 m} \nabla^{2} \varphi_{n}(\mathbf{x})-T_{s}\left(n^{(1)}\right)
$$

In fact, this is shown that the exchange-correlation functional can be written as a functional of the electron density which is available to the seminal paper by Levy [52]. Note that the $\varphi_{n}(\mathbf{x})$ and the $\bar{f}_{n}$ are natural orbitals and occupation of the interacting electron gas, respectively. They are completely different from the Kohn-Sham orbitals $\phi_{n}(\mathbf{x})$ and occupations $f_{n}$.

\section{Total energy and self-consistent equations}

Finally by putting all together, the ground state total energy has the form

$$
\begin{aligned}
E= & \min _{n^{(1)}} \min _{\varphi_{n}, \bar{f}_{n}} \operatorname{stat}\left\{\sum_{v_{e f f}, \Lambda} \bar{f}_{n} \int d^{4} \mathbf{x} \varphi_{n}^{*}(\mathbf{x}) \frac{-\hbar^{2}}{2 m} \nabla^{2} \varphi_{n}(\mathbf{x})\right. \\
& +\int d^{3} \mathbf{r} v_{e f f}(\mathbf{r})\left(\left[\sum_{n} \bar{f}_{n} \sum_{s} \varphi_{n}^{*}(\mathbf{x}) \varphi_{n}(\mathbf{x})\right]-n^{(1)}(\mathbf{r})\right)+\int d^{3} \mathbf{r} v_{e x t}(\mathbf{r}) n^{(1)}(\mathbf{r}) \\
& \left.+\frac{1}{2} \int d^{3} \mathbf{r} \int d^{3} \mathbf{r}^{\prime} \frac{e^{2} n^{(1)}(\mathbf{r}) n^{(1)}\left(\mathbf{r}^{\prime}\right)}{4 \pi \epsilon_{0}\left|\mathbf{r}-\mathbf{r}^{\prime}\right|}+E_{x c}\left[n^{(1)}\right]-\sum_{n, m} \Lambda_{n, m}\left(\left\langle\varphi_{n} \mid \varphi_{m}\right\rangle-\delta_{n, m}\right)\right\}
\end{aligned}
$$

where, the Lagrange multiplier, $\Lambda_{n, m}$, is added for the orthonormality. Diagonalization of this parameter yields a diagonal matrix with the one-particle energies on the diagonal. The effective potential, $v_{e f f}$, is used as a Lagrange multiplier for the density constraint. The minimization of total energy equation leads to Eq. 2.26 which is so-called Kohn-Sham equation. Now, form a set of coupled equations, one can determine the electron density (Eq. A.8), effective potential (Eq. 2.27), and finally total energy (Eq. A.15). These equations can be solved in the so-called self-consistency cycles. Once the set of self-consistent equations has been solved, the electron density and consequently the total energy can be calculated. This strategy has been shown below: 


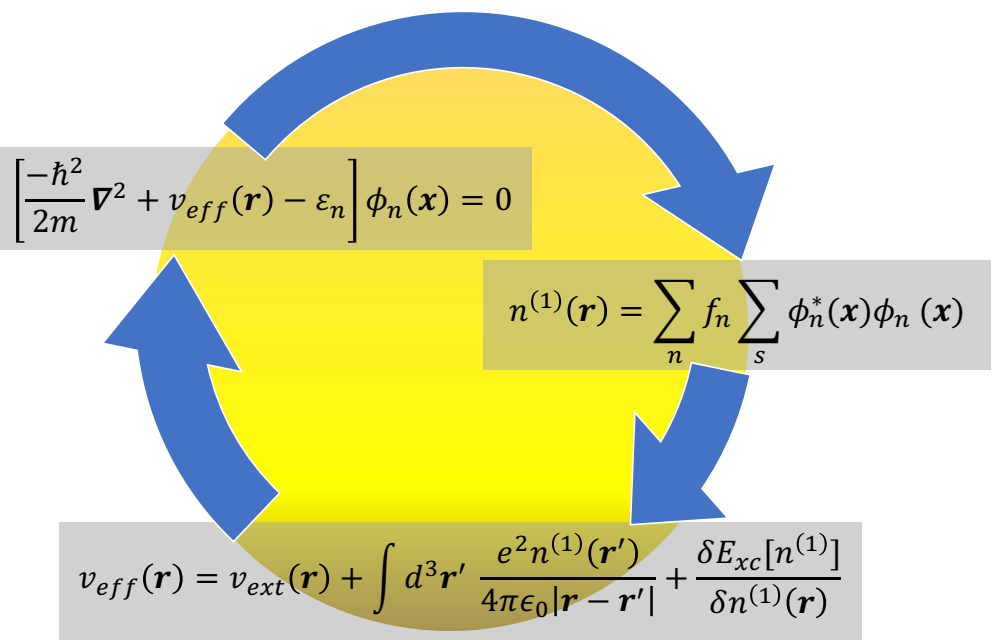

Fig. A.1: Self-consistency cycle. 


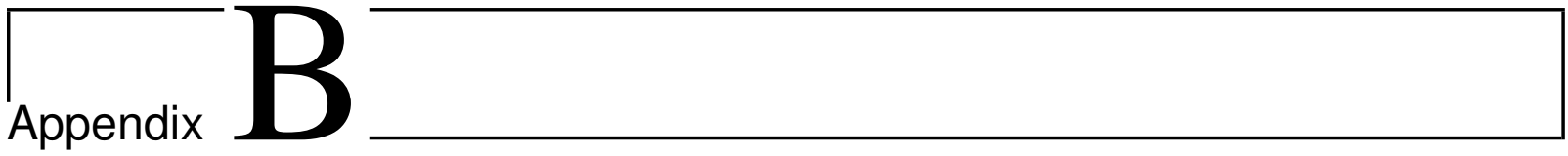

\section{The optimized xyz coordinates}

The optimized $x y z$ coordinates of the calculated adsorbates on $\mathrm{CaMnO}_{3}(001)$ are listed below. The length unit of our system namely 'LUNIT [AA]' is specified to the $3.72633 \AA$. The variable ' $T$ ' in the block 'LATTICE' stands for the lattice vectors $\overrightarrow{t_{1}}, \overrightarrow{t_{2}}, \overrightarrow{t_{3}}$ in the format of $\left(t_{1, x}, t_{1, y}, t_{1, z}, t_{2, x}, t_{2, y}, t_{2, z}, t_{3, x}, t_{3, y}, t_{3, z}\right)$. First line is corresponding to the first lattice vector, second line to the second lattice vector, and third line to the third lattice vector. It should be noted that each branch ends with '!END'. Then, the coordinates are given in units of lattice constants $\AA$. For each atom, there is a branch '! ATOM' which contains '!NAME=' with the first two letters of the element symbol, namely atom types. Next to it, ' $\mathrm{R}=$ ' indicates the atomic positions in Cartesian coordinates in the units specified by 'LUNIT'.

\section{B.1 Water-covered $\mathrm{CaMnO}_{3}(001)$ surface}

In the following the optimized atomic positions which are used in Fig. 4.14 are provided in cartesian coordinates.

\begin{tabular}{|c|c|c|c|c|}
\hline ! LATTICE & $\mathrm{T}=$ & $\begin{array}{l}1.00000 \\
0.00000 \\
1.00000\end{array}$ & $\begin{array}{r}-1.00000 \\
0.00000 \\
1.00000\end{array}$ & $\begin{array}{r}0.00000 \\
10.00000 \\
0.00000\end{array}$ \\
\hline ! ATOM NAME $=$ 'H_011' & $R=$ & 0.04166 & 1.21566 & -1.66600 \\
\hline ! ATOM NAME $=$ 'H_012' & $R=$ & -0.06126 & 0.80686 & -1.70035 \\
\hline ! ATOM NAME $={ }^{\prime}$ O_01' & $R=$ & -0.11907 & 1.02976 & -1.54238 \\
\hline ! ATOM NAME $=$ 'H_021' & $R=$ & 0.02202 & 0.28819 & -1.82384 \\
\hline ! ATOM NAME $=$ 'H_022' & $R=$ & 0.18770 & -0.30548 & -1.82007 \\
\hline ! ATOM NAME $=$ 'O_02' & $R=$ & -0.01631 & -0.02104 & -1.54284 \\
\hline ! ATOM NAME $=$ 'H_031' & $R=$ & 0.58645 & 1.47133 & -1.07574 \\
\hline ! ATOM NAME $=$ 'H_032' & $R=$ & 0.11058 & 0.48790 & -2.22080 \\
\hline
\end{tabular}




\begin{tabular}{|c|c|c|c|c|c|}
\hline ! ATOM NAME $=$ 'O_03' & $R=$ & -0.03299 & 0.50494 & -1.98741 & !END \\
\hline ! ATOM NAME $=$ 'H_041' & $R=$ & -0.26769 & -0.10699 & -1.58345 & !END \\
\hline ! ATOM NAME $=$ 'H_042' & $R=$ & -0.47773 & 0.48498 & -2.00088 & !END \\
\hline ! ATOM NAME = 'O_04' & $R=$ & -0.75234 & 0.48114 & -1.98467 & !EN \\
\hline ! ATOM NAME $=$ 'H_051' & $R=$ & 0.06173 & 1.19324 & -2.96522 & $! \mathrm{El}$ \\
\hline ! ATOM NAME $=$ 'H_052' & $R=$ & 0.12945 & 0.78535 & -2.95850 & !EN \\
\hline ! ATOM NAME $=$ 'O_05' & $R=$ & 0.04579 & 0.97821 & -3.13234 & $! 1$ \\
\hline ! ATOM NAME $={ }^{\prime} \mathrm{H}_{-} 061 '$ & $R=$ & 0.14308 & -0.28822 & -2.80941 & \\
\hline ! ATOM NAME $=$ 'H_062' & $R=$ & 0.11382 & -0.47285 & -2.40981 & !EN \\
\hline ! ATOM NAME $={ }^{\prime}$ O_06' & $R=$ & 0.00261 & -0.46789 & -2.66146 & $! \mathrm{El}$ \\
\hline ! ATOM NAME $={ }^{\prime} \mathrm{H}_{-} 071^{\prime}$ & $R=$ & 0.55973 & 0.55311 & -2.62163 & !EN \\
\hline ! ATOM NAME $={ }^{\prime} \mathrm{H}_{-} 072^{\prime}$ & $R=$ & 0.27815 & 0.29071 & -2.79075 & $! E$ \\
\hline ! ATOM NAME $=$ 'O_07' & $R=$ & 0.28866 & 0.50503 & -2.62298 & !El \\
\hline ! ATOM NAME $=$ 'H_081' & $R=$ & -0.36961 & 0.95886 & -3.15024 & \\
\hline ! ATOM NAME $=$ 'H_082' & $R=$ & -0.75350 & 0.99877 & -3.36406 & !EI \\
\hline ! ATOM NAME $=$ 'O_08' & $R=$ & -0.65124 & 0.97122 & -3.12036 & !E! \\
\hline ! ATOM NAME $={ }^{\prime} \mathrm{CA} 1^{\prime}$ & $R=$ & -0.47364 & 0.41146 & 0.52686 & !EN \\
\hline ! ATOM NAME $={ }^{\prime} \mathrm{CA} 2{ }^{\prime}$ & $R=$ & 0.42147 & 0.52364 & -0.50227 & !EI \\
\hline ! ATOM NAME $={ }^{\prime} \mathrm{CA} 3^{\prime}$ & $R=$ & -0.57444 & 0.55073 & -0.46836 & !EN \\
\hline ! ATOM NAME $=$ 'CA4' & $R=$ & 0.53625 & 0.44052 & 0.55054 & !El \\
\hline ! ATOM NAME $=$ 'MN1' & $R=$ & -0.02066 & 0.98213 & 0.02323 & !EN \\
\hline ! ATOM NAME $=$ 'MN2' & $R=$ & -0.02137 & -0.01676 & 0.02551 & !EN \\
\hline ! ATOM NAME $={ }^{\prime}$ MN3' & $R=$ & -0.02870 & 0.99515 & 1.05100 & !EN \\
\hline ! ATOM NAME $=$ 'MN4' & $R=$ & -0.00744 & -0.03216 & 1.05822 & !EN \\
\hline ! ATOM NAME $=$ 'MN5' & $R=$ & -0.01680 & 1.00917 & -1.01337 & !EN \\
\hline ! ATOM NAME $=$ 'MN6' & $R=$ & -0.03449 & -0.04563 & -1.01967 & !EN \\
\hline ! ATOM NAME $={ }^{\prime} 0_{-}(\mathrm{A}) 1^{\prime}$ & $R=$ & 0.04254 & 0.09179 & 0.54018 & !E! \\
\hline ! ATOM NAME $={ }^{\prime} O_{-}(\mathrm{A}) 2^{\prime}$ & $R=$ & 0.08975 & 1.03549 & -0.49419 & !EN \\
\hline ! ATOM NAME $={ }^{\prime} O_{-}(\mathrm{A}) 3^{\prime}$ & $R=$ & -1.08742 & 0.88091 & -0.48864 & !EN \\
\hline ! ATOM NAME $={ }^{\prime} O_{-}(\mathrm{A}) 4^{\prime}$ & $R=$ & 0.86741 & -0.08477 & 0.54154 & !EN \\
\hline ! ATOM NAME $={ }^{\prime} O_{-}$(B) $1^{\prime}$ & $R=$ & -0.52137 & -0.10810 & 0.13042 & !EN \\
\hline ! ATOM NAME $={ }^{\prime} O_{-}(\mathrm{B}) 2^{\prime}$ & $R=$ & -0.10729 & 0.48229 & -0.08479 & !EN \\
\hline ! ATOM NAME $=10_{-}(\mathrm{B}) 3^{\prime}$ & $R=$ & -0.52956 & 1.11761 & 1.13052 & !EN \\
\hline ! ATOM NAME $=10_{-}(\mathrm{B}) 4$ ' & $R=$ & 1.07065 & 0.48673 & 0.92947 & !EN \\
\hline ! ATOM NAME $=10_{-}$(B) 5 ' & $R=$ & -0.51826 & 1.07847 & -0.07406 & !EN \\
\hline ! ATOM NAME $={ }^{\prime} O_{-}(\mathrm{B}) 6^{\prime}$ & $R=$ & 1.07531 & 0.48234 & 0.11968 & !EN \\
\hline ! ATOM NAME $={ }^{\prime} O_{-}(\mathrm{B}) 7^{\prime}$ & $R=$ & -0.53028 & -0.09404 & 0.95478 & !EN \\
\hline ! ATOM NAME $={ }^{\prime} O_{-}(\mathrm{B}) 8^{\prime}$ & $R=$ & -0.15903 & 0.49303 & 1.16013 & !EN \\
\hline ! ATOM NAME $=10_{-}$(B) 9 ' & $R=$ & -0.51764 & 1.05626 & -0.90087 & !EN \\
\hline ! ATOM NAME $={ }^{\prime} O_{-}$(B) $10^{\prime}$ & $R=$ & 1.16043 & 0.47184 & -1.06467 & !EN \\
\hline ! ATOM NAME $={ }^{\prime} O_{-}$(B) $11^{\prime}$ & $R=$ & -0.52470 & -0.18738 & -1.07842 & !EN \\
\hline ! ATOM NAME $={ }^{\prime} O_{-}$(B) $12^{\prime}$ & $R=$ & -0.15467 & 0.46225 & -0.97375 & !EI \\
\hline ! ATOM NAME $=$ 'H_611' & $R=$ & 0.19554 & 1.21490 & 1.71435 & !E \\
\hline
\end{tabular}




\begin{tabular}{|c|c|c|c|c|c|}
\hline ! ATOM NAME $=$ 'H_612' & $R=$ & 0.06871 & 0.80188 & 1.70646 & !END \\
\hline ! ATOM NAME= '0_61' & $R=$ & 0.01887 & 1.04712 & 1.58713 & !END \\
\hline ! ATOM NAME $=$ 'H_621' & $R=$ & 0.04969 & 0.21475 & 1.81706 & !END \\
\hline ! ATOM NAME $=$ 'H_622' & $R=$ & 0.25739 & -0.31471 & 1.85258 & !END \\
\hline ! ATOM NAME $={ }^{\prime}$ O_62' & $R=$ & -0.05279 & -0.13600 & 1.57107 & ! ENI \\
\hline ! ATOM NAME $={ }^{\prime} \mathrm{H}_{-} 631$ ' & $R=$ & 0.58393 & 1.49864 & 1.23437 & !E \\
\hline ! ATOM NAME $=$ 'H_632' & $R=$ & 0.19073 & 0.42464 & 2.19332 & \\
\hline ! ATOM NAME $={ }^{\prime} 0 \_63^{\prime}$ & $R=$ & 0.08783 & 0.45688 & 1.94141 & $! E$ \\
\hline ! ATOM NAME $=$ 'H_641' & $R=$ & -0.27485 & -0.28325 & 1.59885 & $! E$ \\
\hline ! ATOM NAME $=$ 'H_642' & $R=$ & -0.35879 & 0.51759 & 1.99854 & !EN \\
\hline ! ATOM NAME = 'O_64' & $R=$ & -0.63160 & 0.49457 & 2.01636 & \\
\hline ! ATOM NAME $=$ 'H_651' & $R=$ & 0.09373 & 1.18097 & 2.96316 & !EN \\
\hline ! ATOM NAME $=$ 'H_652' & $R=$ & 0.15747 & 0.77257 & 2.94974 & !EI \\
\hline ! ATOM NAME $=$ '0_65' & $R=$ & 0.07436 & 0.96297 & 3.12630 & !EN \\
\hline ! ATOM NAME $=$ 'H_661' & $R=$ & 0.17130 & -0.29602 & 2.82578 & !EN \\
\hline ! ATOM NAME $=$ 'H_662' & $R=$ & 0.18721 & -0.47977 & 2.43014 & !EN \\
\hline ! ATOM NAME $={ }^{\prime} 0 \_66^{\prime}$ & $R=$ & 0.04418 & -0.47446 & 2.66411 & !EN \\
\hline ! ATOM NAME $=$ 'H_671' & $R=$ & 0.59534 & 0.54078 & 2.61463 & !EN \\
\hline ! ATOM NAME $=$ 'H_672' & $R=$ & 0.31580 & 0.28206 & 2.79179 & !EN \\
\hline ! ATOM NAME = 'O_67' & $R=$ & 0.32517 & 0.49064 & 2.61671 & !EN \\
\hline ! ATOM NAME $=$ 'H_681' & $R=$ & -0.34250 & 0.95134 & 3.15061 & !EN \\
\hline ! ATOM NAME $=$ 'H_682' & $R=$ & -0.72076 & 1.00935 & 3.37041 & !EN \\
\hline ! ATOM NAME $=10 \_68$ ' & $R=$ & -0.62424 & 0.97031 & 3.12587 & ס \\
\hline
\end{tabular}

\section{B.2 First Proton-Electron Transfer}

In the following the optimized atomic positions which are used in Fig. 4.18b are provided in cartesian coordinates.

\begin{tabular}{|c|c|c|c|c|c|}
\hline ! LATTICE & $\mathrm{T}=$ & 1.00000 & -1.00000 & 0.00000 & \\
\hline & & 0.00000 & 0.00000 & 10.00000 & \\
\hline & & 1.00000 & 1.00000 & 0.00000 & !END \\
\hline ! ATOM NAME $=$ 'H_011' & $R=$ & 0.04493 & 1.17834 & -1.69026 & !END \\
\hline ! ATOM NAME $=$ 'H_012' & $R=$ & -0.04236 & 0.75363 & -1.66450 & !END \\
\hline ! ATOM NAME $=$ 'O_01' & $R=$ & -0.10041 & 0.99690 & -1.53663 & !END \\
\hline ! ATOM NAME $=$ 'H_021' & $R=$ & 0.08559 & 0.36215 & -2.14478 & !END \\
\hline ! ATOM NAME $=$ 'H_022' & $R=$ & 0.19134 & -0.33170 & -1.84592 & !END \\
\hline ! ATOM NAME $=$ 'O_02' & $R=$ & 0.01834 & -0.01222 & -1.55612 & !END \\
\hline ! ATOM NAME $=$ 'H_031' & $R=$ & 0.61146 & 1.48849 & -1.07737 & !END \\
\hline ! ATOM NAME = 'O_03' & $R=$ & -0.06357 & 0.43022 & -1.92622 & !END \\
\hline ! ATOM NAME $=$ 'H_041' & $R=$ & -0.23977 & -0.07113 & -1.60345 & !END \\
\hline
\end{tabular}




\begin{tabular}{|c|c|c|c|c|c|}
\hline ! ATOM NAME $=$ 'H_042' & $R=$ & -0.49003 & 0.41641 & -1.98646 & !END \\
\hline ! ATOM NAME $=$ 'O_04' & $R=$ & -0.76724 & 0.43851 & -1.98894 & !END \\
\hline ! ATOM NAME $=$ 'H_051' & $R=$ & -0.01712 & 1.08674 & -2.95877 & !END \\
\hline ! ATOM NAME $=$ 'H_052' & $R=$ & 0.04706 & 0.67773 & -2.94215 & !ENI \\
\hline ! ATOM NAME $=$ '0_05' & $R=$ & -0.03305 & 0.86847 & -3.12052 & $! \mathrm{El}$ \\
\hline ! ATOM NAME $={ }^{\prime} \mathrm{H}_{-} 061^{\prime}$ & $R=$ & 0.05551 & -0.38744 & -2.82365 & !EN \\
\hline ! ATOM NAME $={ }^{\prime} \mathrm{H}_{-} 062$ ' & $R=$ & 0.05672 & -0.54864 & -2.41839 & $! 1$ \\
\hline ! ATOM NAME $={ }^{\prime}$ O_06' & $R=$ & -0.07742 & -0.55649 & -2.65628 & \\
\hline ! ATOM NAME $=$ 'H_071' & $R=$ & 0.47680 & 0.44797 & -2.61557 & !EN \\
\hline ! ATOM NAME $=$ 'H_072' & $R=$ & 0.19688 & 0.17998 & -2.77584 & $! \mathrm{El}$ \\
\hline ! ATOM NAME $=$ 'O_07' & $R=$ & 0.20728 & 0.39441 & -2.60961 & !EN \\
\hline ! ATOM NAME $={ }^{\prime} \mathrm{H}_{-} 081^{\prime}$ & $R=$ & -0.45097 & 0.84822 & -3.14993 & $! E$ \\
\hline ! ATOM NAME $=$ 'H_082' & $R=$ & -0.82711 & 0.90418 & -3.37227 & !El \\
\hline ! ATOM NAME= '0_08' & $R=$ & -0.73266 & 0.85799 & -3.12827 & $! E !$ \\
\hline ! $\mathrm{ATOM}$ NAME $=$ 'CA1' & $R=$ & -0.43783 & 0.43234 & 0.52470 & !EI \\
\hline ! ATOM NAME $={ }^{\prime}$ CA2 ${ }^{\prime}$ & $R=$ & 0.44855 & 0.54234 & -0.50436 & !E! \\
\hline ! ATOM NAME $={ }^{\prime} \mathrm{CA} 3^{\prime}$ & $R=$ & -0.54768 & 0.56801 & -0.47120 & !EN \\
\hline ! ATOM NAME $={ }^{\prime} \mathrm{CA} 4{ }^{\prime}$ & $R=$ & 0.57529 & 0.46111 & 0.55170 & !EI \\
\hline ! ATOM NAME $={ }^{\prime} M N 1{ }^{\prime}$ & $R=$ & 0.01010 & 1.00096 & 0.02294 & !EN \\
\hline ! ATOM NAME $=$ 'MN2' & $R=$ & 0.01006 & 0.00142 & 0.02355 & ! El \\
\hline ! ATOM NAME = 'MN3' & $R=$ & 0.01398 & 1.01423 & 1.05649 & !EN \\
\hline ! ATOM NAME = 'MN4' & $R=$ & 0.03141 & 0.00001 & 1.06441 & !EN \\
\hline ! ATOM NAME $=$ 'MN5' & $R=$ & 0.00789 & 1.02408 & -1.01674 & !EN \\
\hline ! ATOM NAME = 'MN6' & $R=$ & -0.00792 & -0.02543 & -1.02665 & !EN \\
\hline ! ATOM NAME $={ }^{\prime} O_{-}(\mathrm{A}) 1^{\prime}$ & $R=$ & 0.08295 & 0.10879 & 0.53919 & !EN \\
\hline ! ATOM NAME $=10_{-}(\mathrm{A}) 2^{\prime}$ & $R=$ & 0.11562 & 1.05418 & -0.49487 & !EN \\
\hline ! ATOM NAME $=10_{-}(\mathrm{A}) 3^{\prime}$ & $R=$ & -1.05978 & 0.89730 & -0.49380 & !EI \\
\hline ! ATOM NAME $={ }^{\prime} O_{-}(\mathrm{A}) 4$ ' & $R=$ & 0.89917 & -0.05719 & 0.54583 & !EN \\
\hline ! ATOM NAME $={ }^{\prime} O_{-}(\mathrm{B}) 1^{\prime}$ & $R=$ & -0.49051 & -0.09407 & 0.12419 & !EN \\
\hline ! ATOM NAME $={ }^{\prime} O_{-}(\mathrm{B}) 2^{\prime}$ & $R=$ & -0.08222 & 0.49942 & -0.08219 & !EN \\
\hline ! ATOM NAME $={ }^{\prime} O_{-}$(B) 3 ' & $R=$ & -0.48727 & 1.14729 & 1.14291 & !EN \\
\hline ! ATOM NAME $=1 O_{-}(\mathrm{B}) 4^{\prime}$ & $R=$ & 1.12284 & 0.51296 & 0.96283 & !EN \\
\hline ! ATOM NAME $=10_{-}(\mathrm{B}) 5^{\prime}$ & $R=$ & -0.48811 & 1.09556 & -0.07637 & !EN \\
\hline ! ATOM NAME $={ }^{\prime} 0_{-}(\mathrm{B}) 6^{\prime}$ & $R=$ & 1.10733 & 0.50067 & 0.12106 & !EN \\
\hline ! ATOM NAME $=10_{-}(\mathrm{B}) 7^{\prime}$ & $R=$ & -0.48839 & -0.06913 & 0.93530 & !EN \\
\hline ! ATOM NAME $={ }^{\prime} O_{-}(\mathrm{B}) 8$ ' & $R=$ & -0.13323 & 0.51680 & 1.13416 & !EN \\
\hline ! ATOM NAME $={ }^{\prime} O_{-}(\mathrm{B}) 9^{\prime}$ & $R=$ & -0.49282 & 1.07233 & -0.90105 & !EN \\
\hline ! ATOM NAME $={ }^{\prime} O_{-}$(B) $10^{\prime}$ & $R=$ & 1.18293 & 0.48786 & -1.07443 & !EN \\
\hline ! ATOM NAME $={ }^{\prime} O_{-}$(B) $11^{\prime}$ & $R=$ & -0.49684 & -0.16691 & -1.08069 & !EN \\
\hline ! ATOM NAME $={ }^{\prime} O_{-}$(B) $12^{\prime}$ & $R=$ & -0.13035 & 0.47941 & -0.97198 & !EN \\
\hline ! ATOM NAME $=$ 'H_611' & $R=$ & 0.13509 & 1.18999 & 1.76732 & !EN \\
\hline ! ATOM NAME $=$ 'H_612' & $R=$ & 0.06737 & 0.74685 & 1.70381 & !EN \\
\hline ! ATOM NAME $=$ 'O_61' & $R=$ & 0.16884 & 0.98281 & 1.58152 & !E \\
\hline
\end{tabular}




\begin{tabular}{|c|c|c|c|c|c|}
\hline ! ATOM NAME $=$ 'H_621' & $R=$ & 0.04424 & 0.34153 & 2.13053 & !END \\
\hline ! ATOM NAME $=$ 'H_622' & $R=$ & 0.11701 & -0.29909 & 1.90100 & !END \\
\hline ! ATOM NAME $={ }^{\prime} 0 \_62 '$ & $R=$ & -0.07259 & -0.05402 & 1.58157 & !EN \\
\hline ! ATOM NAME $=$ 'H_631' & $R=$ & 0.60940 & 1.52223 & 1.21129 & !END \\
\hline ! ATOM NAME $=$ '0_63' & $R=$ & -0.09714 & 0.42451 & 1.91299 & ! \\
\hline ! ATOM NAME $=$ 'H_641 ' & $R=$ & -0.34467 & -0.06456 & 1.59451 & !E \\
\hline ! ATOM NAME $=$ 'H_642' & $R=$ & -0.52058 & 0.48769 & 2.02618 & $! \mathrm{E}$ \\
\hline ! ATOM NAME $=$ 'O_64' & $R=$ & -0.79561 & 0.49529 & 2.06034 & \\
\hline ! ATOM NAME $={ }^{\prime} H_{-} 651$ ' & $R=$ & -0.01743 & 1.07799 & 2.98962 & !E \\
\hline ! ATOM NAME $=$ 'H_652' & $R=$ & 0.01661 & 0.66693 & 2.92871 & ! El \\
\hline ! ATOM NAME $=$ '0_65' & $R=$ & -0.04657 & 0.84453 & 3.12602 & !E \\
\hline ! ATOM NAME $={ }^{\prime} H_{-} 661$ ' & $R=$ & 0.01265 & -0.35382 & 2.89764 & !El \\
\hline ! ATOM NAME $=$ 'H_662' & $R=$ & 0.04608 & -0.50748 & 2.49315 & $! E$ \\
\hline ! ATOM NAME $=$ '0_66' & $R=$ & -0.09350 & -0.53547 & 2.72608 & !El \\
\hline ! ATOM NAME $={ }^{\prime} H_{-} 671$ ' & $R=$ & 0.46020 & 0.45182 & 2.62546 & !E \\
\hline ! ATOM NAME $=$ 'H_672' & $R=$ & 0.19237 & 0.17567 & 2.78176 & !EN \\
\hline ! ATOM NAME = 'O_67' & $R=$ & 0.19688 & 0.38024 & 2.60278 & $!$ El \\
\hline ! ATOM NAME $=$ 'H_681' & $R=$ & -0.47196 & 0.87763 & 3.18120 & !El \\
\hline ! ATOM NAME $=$ 'H_682' & $R=$ & -0.80407 & 1.08023 & 3.38098 & !EN \\
\hline ! ATOM NAME $={ }^{\prime} 0 \_68$ ' & $R=$ & -0.75004 & 0.91459 & 3.17994 & !El \\
\hline
\end{tabular}

\section{B.3 Second Proton-Electron Transfer}

In the following the optimized atomic positions which are used in Fig. 4.19b are provided in cartesian coordinates.

\begin{tabular}{|c|c|c|c|c|}
\hline ! LATTICE & $\mathrm{T}=$ & $\begin{array}{l}1.00000 \\
0.00000 \\
1.00000\end{array}$ & $\begin{array}{r}-1.00000 \\
0.00000 \\
1.00000\end{array}$ & $\begin{array}{r}0.00000 \\
10.00000 \\
0.00000\end{array}$ \\
\hline ! ATOM NAME $=$ 'H_011' & $R=$ & 0.04678 & 1.16834 & -1.68747 \\
\hline ! ATOM NAME $=$ 'H_012' & $R=$ & -0.02431 & 0.74689 & -1.59606 \\
\hline ! ATOM NAME $=$ 'O_01' & $R=$ & -0.11373 & 0.99880 & -1.53623 \\
\hline ! ATOM NAME $=$ 'H_021' & $R=$ & 0.11486 & 0.29968 & -1.99008 \\
\hline ! ATOM NAME $=$ 'H_022' & $R=$ & 0.20773 & -0.33190 & -1.80867 \\
\hline ! ATOM NAME= 'O_02' & $R=$ & 0.03697 & -0.00254 & -1.56246 \\
\hline ! ATOM NAME $=$ 'H_031' & $R=$ & 0.59218 & 1.52023 & -1.08094 \\
\hline ! ATOM NAME = 'O_03' & $R=$ & -0.03593 & 0.32471 & -1.76024 \\
\hline ! ATOM NAME $=$ 'H_042' & $R=$ & -0.47142 & 0.38768 & -1.89180 \\
\hline ! ATOM NAME = 'O_04' & $R=$ & -0.73352 & 0.43895 & -1.95045 \\
\hline ! ATOM NAME $=$ 'H_051' & $R=$ & 0.01974 & 1.04014 & -2.86958 \\
\hline ! ATOM NAME = 'H_052' & $R=$ & 0.05034 & 0.63444 & -2.79221 \\
\hline
\end{tabular}




\begin{tabular}{|c|c|c|c|c|c|}
\hline ! ATOM NAME $=$ 'O_05' & $R=$ & -0.01025 & 0.80160 & -2.99788 & !END \\
\hline ! ATOM NAME $=$ 'H_061' & $R=$ & 0.04252 & -0.38845 & -2.76358 & !END \\
\hline ! ATOM NAME $=$ 'H_062' & $R=$ & 0.09550 & -0.56229 & -2.37074 & $! E$ \\
\hline ! ATOM NAME $=$ '0_06' & $R=$ & -0.05853 & -0.57766 & -2.59700 & !EN \\
\hline ! ATOM NAME $=$ 'H_071' & $R=$ & 0.50239 & 0.42762 & -2.46591 & $! \mathrm{El}$ \\
\hline ! ATOM NAME $={ }^{\prime} \mathrm{H}_{-} 072^{\prime}$ & $R=$ & 0.22653 & 0.15124 & -2.60407 & . \\
\hline ! ATOM NAME $=$ 'O_07' & $R=$ & 0.23765 & 0.36247 & -2.43367 & \\
\hline ! ATOM NAME $=$ 'H_081' & $R=$ & -0.44245 & 0.84856 & -3.03372 & \\
\hline ! ATOM NAME $=$ 'H_082' & $R=$ & -0.77119 & 1.06932 & -3.21541 & $! E$ \\
\hline ! ATOM NAME $=$ 'O_08' & $R=$ & -0.71712 & 0.89750 & -3.01946 & \\
\hline ! ATOM NAME $={ }^{\prime} \mathrm{CA} 1^{\prime}$ & $R=$ & -0.45577 & 0.46206 & 0.52285 & !EN \\
\hline ! ATOM NAME $={ }^{\prime} \mathrm{CA} 2{ }^{\prime}$ & $R=$ & 0.43549 & 0.57440 & -0.50411 & $! E$ \\
\hline ! ATOM NAME $=$ 'CA3' & $R=$ & -0.56273 & 0.60165 & -0.47411 & $! E$ \\
\hline ! ATOM NAME $=$ 'CA4' & $R=$ & 0.55367 & 0.49367 & 0.55285 & \\
\hline ! ATOM NAME $=$ 'MN1' & $R=$ & -0.00690 & 1.03313 & 0.02167 & !El \\
\hline ! ATOM NAME $=$ 'MN2' & $R=$ & -0.00691 & 0.03320 & 0.02139 & !El \\
\hline ! ATOM NAME = 'MN3' & $R=$ & -0.00649 & 1.04375 & 1.05460 & !E! \\
\hline ! ATOM NAME $=$ 'MN4' & $R=$ & 0.01803 & 0.03117 & 1.07133 & $! \mathrm{f}$ \\
\hline ! ATOM NAME $=$ 'MN5' & $R=$ & -0.00674 & 1.05525 & -1.01218 & !ENI \\
\hline ! ATOM NAME $=$ 'MN6' & $R=$ & -0.02374 & 0.00457 & -1.03502 & !El \\
\hline ! ATOM NAME $={ }^{\prime} 0_{-}(\mathrm{A}) 1^{\prime}$ & $R=$ & 0.05937 & 0.13793 & 0.53833 & $! \mathrm{El}$ \\
\hline ! ATOM NAME $={ }^{\prime} 0_{-}(\mathrm{A}) 2^{\prime}$ & $R=$ & 0.10160 & 1.08577 & -0.49560 & !EN \\
\hline ! ATOM NAME $={ }^{\prime} O_{-}(\mathrm{A}) 3^{\prime}$ & $R=$ & -1.07464 & 0.92968 & -0.49707 & !E! \\
\hline ! ATOM NAME $={ }^{\prime} O_{-}(\mathrm{A}) 4^{\prime}$ & $R=$ & 0.88268 & -0.02272 & 0.54400 & !EN \\
\hline ! ATOM NAME $={ }^{\prime} O_{-}(\mathrm{B}) 1^{\prime}$ & $R=$ & -0.50813 & -0.06082 & 0.12442 & !EN \\
\hline ! ATOM NAME $={ }^{\prime} O_{-}$(B) $2^{\prime}$ & $R=$ & -0.09703 & 0.53158 & -0.08619 & !EN \\
\hline ! ATOM NAME $={ }^{\prime} O_{-}$(B) 3 ' & $R=$ & -0.50400 & 1.18580 & 1.13085 & !E! \\
\hline ! ATOM NAME $={ }^{\prime} O_{-}(\mathrm{B}) 4$ ' & $R=$ & 1.11606 & 0.54433 & 0.97494 & !EN \\
\hline ! ATOM NAME $={ }^{\prime} O_{-}(\mathrm{B}) 5^{\prime}$ & $R=$ & -0.50448 & 1.12673 & -0.07667 & !EN \\
\hline ! ATOM NAME $={ }^{\prime} O_{-}(\mathrm{B}) 6^{\prime}$ & $R=$ & 1.08974 & 0.53271 & 0.11876 & !EI \\
\hline ! ATOM NAME $={ }^{\prime} O_{-}(\mathrm{B}) 7^{\prime}$ & $R=$ & -0.50988 & -0.04602 & 0.95396 & !EN \\
\hline ! ATOM NAME $={ }^{\prime} O_{-}(\mathrm{B}) 8^{\prime}$ & $R=$ & -0.15531 & 0.54419 & 1.13592 & !EN \\
\hline ! ATOM NAME $={ }^{\prime} O_{-}$(B) $9 '$ & $R=$ & -0.50740 & 1.10539 & -0.89714 & !EN \\
\hline ! ATOM NAME $={ }^{\prime} O_{-}$(B) $10^{\prime}$ & $R=$ & 1.16194 & 0.52042 & -1.08636 & !EN \\
\hline ! ATOM NAME $={ }^{\prime} O_{-}$(B) $11^{\prime}$ & $R=$ & -0.51620 & -0.13119 & -1.08073 & !EN \\
\hline ! ATOM NAME $={ }^{\prime} O_{-}$(B) $12^{\prime}$ & $R=$ & -0.14797 & 0.51044 & -0.98069 & !EN \\
\hline ! ATOM NAME $=$ 'H_611' & $R=$ & 0.20413 & 1.20579 & 1.73938 & !EN \\
\hline ! ATOM NAME $=$ 'H_612' & $R=$ & 0.18659 & 0.77943 & 1.63372 & !EN \\
\hline ! ATOM NAME $=$ '0_61' & $R=$ & 0.06066 & 1.01531 & 1.59608 & !EN \\
\hline ! ATOM NAME $=$ 'H_621' & $R=$ & 0.15165 & 0.22241 & 2.03154 & !EN \\
\hline ! ATOM NAME $=$ 'H_622' & $R=$ & 0.25210 & -0.28847 & 1.81667 & !EN \\
\hline ! ATOM NAME $={ }^{\prime}$ O_62' & $R=$ & -0.03874 & -0.03073 & 1.59961 & !E! \\
\hline ! ATOM NAME $=$ 'H_631' & $R=$ & 0.57941 & 1.54651 & 1.18687 & !E] \\
\hline
\end{tabular}




\begin{tabular}{|c|c|c|c|c|c|}
\hline ! ATOM NAME= '0_63' & $R=$ & 0.03476 & 0.29934 & 1.79539 & ! END \\
\hline ! ATOM NAME $=$ 'H_642' & $R=$ & -0.37548 & 0.49490 & 1.92298 & !E! \\
\hline ! ATOM NAME $=$ '0_64' & $R=$ & -0.63974 & 0.52113 & 1.98505 & \\
\hline ! ATOM NAME $=$ 'H_651' & $R=$ & 0.04194 & 1.04364 & 2.87427 & \\
\hline ! ATOM NAME $=$ 'H_652' & $R=$ & 0.07280 & 0.63361 & 2.81004 & \\
\hline ! ATOM NAME $=$ 'O_65' & $R=$ & 0.00617 & 0.80916 & 3.00784 & \\
\hline ! ATOM NAME $=$ 'H_661' & $R=$ & 0.07343 & -0.38278 & 2.79530 & \\
\hline ! ATOM NAME $=$ 'H_662' & $R=$ & 0.14903 & -0.52519 & 2.39705 & \\
\hline ! ATOM NAME= 'O_66' & $R=$ & -0.01535 & -0.56152 & 2.61147 & !E \\
\hline ! ATOM NAME $=$ 'H_671' & $R=$ & 0.53201 & 0.41864 & 2.50251 & !E \\
\hline ! ATOM NAME $=$ 'H_672' & $R=$ & 0.26622 & 0.15061 & 2.67875 & $! E$ \\
\hline ! ATOM NAME= 'O_67' & $R=$ & 0.26878 & 0.34561 & 2.48855 & !E! \\
\hline ! ATOM NAME $=$ 'H_681' & $R=$ & -0.41624 & 0.84888 & 3.06997 & \\
\hline ! ATOM NAME $=$ 'H_682' & $R=$ & -0.74065 & 1.05642 & 3.27761 & 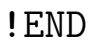 \\
\hline OM & $R=$ & -0.6 & 8 & 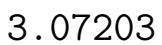 & \\
\hline
\end{tabular}

\section{B.4 Third Proton-Electron Transfer}

In the following the optimized atomic positions which are used in Fig. 4.23 are provided in cartesian coordinates.

\begin{tabular}{|c|c|c|c|c|c|}
\hline ! LATTICE & $\mathrm{T}=$ & $\begin{array}{l}1.00000 \\
0.00000 \\
1.00000\end{array}$ & $\begin{array}{r}-1.00000 \\
0.00000 \\
1.00000\end{array}$ & $\begin{array}{r}0.00000 \\
10.00000 \\
0.00000\end{array}$ & !END \\
\hline ! ATOM NAME $=$ 'H_011' & $R=$ & 0.05511 & 1.14542 & -1.69502 & !END \\
\hline ! ATOM NAME $=$ 'H_012' & $R=$ & -0.00273 & 0.72638 & -1.58969 & !END \\
\hline ! ATOM NAME $=$ 'O_01' & $R=$ & -0.10080 & 0.97912 & -1.53745 & !END \\
\hline ! ATOM NAME $=$ 'H_022' & $R=$ & 0.22771 & -0.34208 & -1.85578 & !END \\
\hline ! ATOM NAME= 'O_02' & $R=$ & 0.05157 & 0.01553 & -1.56864 & !END \\
\hline ! ATOM NAME $=$ 'H_031' & $R=$ & 0.60360 & 1.51172 & -1.07753 & !END \\
\hline ! ATOM NAME $={ }^{\prime}$ O_03' & $R=$ & 0.09838 & 0.31808 & -1.74509 & !END \\
\hline ! ATOM NAME $=$ 'H_042' & $R=$ & -0.49033 & 0.39742 & -2.04046 & !END \\
\hline ! ATOM NAME= 'O_04' & $R=$ & -0.75239 & 0.42318 & -1.98948 & !END \\
\hline ! ATOM NAME $=$ 'H_051' & $R=$ & -0.09431 & 1.13826 & -2.95835 & !END \\
\hline ! ATOM NAME $=$ 'H_052' & $R=$ & -0.02857 & 0.72827 & -2.94400 & !END \\
\hline ! ATOM NAME= 'O_05' & $R=$ & -0.10374 & 0.92254 & -3.12312 & !END \\
\hline ! ATOM NAME $=$ 'H_061' & $R=$ & -0.02174 & -0.34315 & -2.80725 & !END \\
\hline ! ATOM NAME $=$ 'H_062' & $R=$ & -0.00319 & -0.52518 & -2.41984 & !END \\
\hline ! ATOM NAME $={ }^{\prime}$ O_06' & $R=$ & -0.15886 & -0.50897 & -2.63918 & !END \\
\hline ! ATOM NAME $=$ 'H_071' & $R=$ & 0.36438 & 0.48786 & -2.58945 & !END \\
\hline ! ATOM NAME $=$ 'H_072' & $R=$ & 0.10744 & 0.23032 & -2.79155 & ! ENI \\
\hline
\end{tabular}




\begin{tabular}{|c|c|c|c|c|c|}
\hline ! ATOM NAME $=$ 'O_07' & $R=$ & 0.09964 & 0.43345 & -2.61274 & !END \\
\hline ! ATOM NAME $=$ 'H_081' & $R=$ & -0.52387 & 0.89126 & -3.14950 & !END \\
\hline ! ATOM NAME $=$ 'H_082' & $R=$ & -0.90202 & 0.89350 & -3.37419 & !END \\
\hline ! ATOM NAME = 'O_08' & $R=$ & -0.80544 & 0.89558 & -3.12671 & ! ENI \\
\hline ! ATOM NAME $={ }^{\prime} \mathrm{CA} 1{ }^{\prime}$ & $R=$ & -0.45125 & 0.45831 & 0.53579 & $! E$ \\
\hline ! ATOM NAME $=$ 'CA2' & $R=$ & 0.44393 & 0.56544 & -0.50297 & \\
\hline ! ATOM NAME $=$ 'CA3' & $R=$ & -0.55074 & 0.59533 & -0.46867 & !E \\
\hline ! ATOM NAME $=$ 'CA4' & $R=$ & 0.56178 & 0.48848 & 0.55479 & \\
\hline ! ATOM NAME = 'MN1' & $R=$ & 0.00366 & 1.02791 & 0.02403 & $! \mathrm{El}$ \\
\hline ! ATOM NAME $=$ 'MN2' & $R=$ & 0.00256 & 0.02877 & 0.02749 & \\
\hline ! ATOM NAME $={ }^{\prime}$ MN3' & $R=$ & 0.00187 & 1.04086 & 1.05485 & $! \mathrm{El}$ \\
\hline ! ATOM NAME $=$ 'MN4' & $R=$ & 0.02122 & 0.01769 & 1.06365 & $! E$ \\
\hline ! ATOM NAME $=$ 'MN5' & $R=$ & 0.00487 & 1.04933 & -1.01315 & !E \\
\hline ! ATOM NAME = 'MN6' & $R=$ & -0.01432 & -0.00363 & -1.02032 & \\
\hline ! ATOM NAME $=' 0_{-}(\mathrm{A}) 1^{\prime}$ & $R=$ & 0.06792 & 0.14110 & 0.53708 & $! \mathrm{El}$ \\
\hline ! ATOM NAME $={ }^{\prime} 0_{-}(\mathrm{A}) 2^{\prime}$ & $R=$ & 0.11508 & 1.08224 & -0.49485 & !El \\
\hline ! ATOM NAME $={ }^{\prime} O_{-}(\mathrm{A}) 3^{\prime}$ & $R=$ & -1.06658 & 0.91736 & -0.48668 & !E! \\
\hline ! ATOM NAME $={ }^{\prime} O_{-}(\mathrm{A}) 4^{\prime}$ & $R=$ & 0.89065 & -0.03654 & 0.54946 & !EI \\
\hline ! ATOM NAME $={ }^{\prime} O_{-}(\mathrm{B}) 1^{\prime}$ & $R=$ & -0.49878 & -0.05613 & 0.13585 & !EI \\
\hline ! ATOM NAME $={ }^{\prime} O_{-}$(B) $2^{\prime}$ & $R=$ & -0.07814 & 0.52786 & -0.09215 & !EI \\
\hline ! ATOM NAME $=10_{-}$(B) 3 ' & $R=$ & -0.50101 & 1.15705 & 1.13835 & $! E !$ \\
\hline ! ATOM NAME $=10_{-}(\mathrm{B}) 4$ ' & $R=$ & 1.09283 & 0.53363 & 0.94453 & !EN \\
\hline ! ATOM NAME $={ }^{\prime} O_{-}$(B) 5 ' & $R=$ & -0.49327 & 1.11742 & -0.07538 & !E! \\
\hline ! ATOM NAME $={ }^{\prime} O_{-}(\mathrm{B}) 6^{\prime}$ & $R=$ & 1.09261 & 0.52860 & 0.12945 & !EN \\
\hline ! ATOM NAME $={ }^{\prime} O_{-}(\mathrm{B}) 7^{\prime}$ & $R=$ & -0.50016 & -0.04628 & 0.95391 & !EN \\
\hline ! ATOM NAME $=10_{-}(\mathrm{B}) 8^{\prime}$ & $R=$ & -0.12750 & 0.53595 & 1.15738 & !EN \\
\hline ! ATOM NAME $=10_{-}$(B) $9 '$ & $R=$ & -0.49648 & 1.09681 & -0.89887 & !E! \\
\hline ! ATOM NAME $={ }^{\prime} O_{-}$(B) $10^{\prime}$ & $R=$ & 1.17017 & 0.51272 & -1.09069 & !E! \\
\hline ! ATOM NAME $={ }^{\prime} O_{-}$(B) $11^{\prime}$ & $R=$ & -0.50235 & -0.14093 & -1.08326 & !EN \\
\hline ! ATOM NAME $={ }^{\prime} O_{-}$(B) $12^{\prime}$ & $R=$ & -0.13868 & 0.50223 & -0.97230 & $! \mathrm{El}$ \\
\hline ! ATOM NAME $=$ 'H_611' & $R=$ & 0.24574 & 1.22445 & 1.75230 & !EN \\
\hline ! ATOM NAME $=$ 'H_612' & $R=$ & 0.25438 & 0.80921 & 1.63743 & !EN \\
\hline ! ATOM NAME $=$ '0_61' & $R=$ & 0.11065 & 1.03432 & 1.60593 & !EN \\
\hline ! ATOM NAME = 'H_622' & $R=$ & 0.37256 & -0.25049 & 2.03618 & !EN \\
\hline ! ATOM NAME= '0_62' & $R=$ & -0.02051 & -0.08071 & 1.57534 & !EN \\
\hline ! ATOM NAME $=$ 'H_631' & $R=$ & 0.62921 & 1.54709 & 1.26737 & !EN \\
\hline ! ATOM NAME = '0_63' & $R=$ & 0.15560 & 0.12945 & 1.79876 & !EN \\
\hline ! ATOM NAME $=$ 'H_642' & $R=$ & -0.36075 & 0.46511 & 2.21999 & !EN \\
\hline ! ATOM NAME $=$ 'O_64' & $R=$ & -0.60488 & 0.48830 & 2.10312 & !EN \\
\hline ! ATOM NAME $=$ 'H_651' & $R=$ & -0.16750 & 1.14663 & 2.90472 & !EN \\
\hline ! ATOM NAME $=$ 'H_652' & $R=$ & -0.09823 & 0.73997 & 2.89675 & !EN \\
\hline ! ATOM NAME = '0_65' & $R=$ & -0.18408 & 0.93157 & 3.07097 & !EN \\
\hline ! ATOM NAME $=$ 'H_661' & $R=$ & -0.10531 & -0.34219 & 2.75501 & !EI \\
\hline
\end{tabular}




\begin{tabular}{|c|c|c|c|c|}
\hline ! ATOM NAME $=$ 'H_662' & $R=$ & -0.00356 & -0.53544 & 2.39501 \\
\hline ! ATOM NAME= '0_66' & $R=$ & -0.20724 & -0.51816 & 2.57342 \\
\hline ! ATOM NAME= 'H_671' & $R=$ & 0.29997 & 0.49799 & 2.50306 \\
\hline ! ATOM NAME $=$ 'H_672' & $R=$ & 0.04680 & 0.24662 & 2.72336 \\
\hline ! ATOM NAME = '0_67' & $R=$ & 0.03605 & 0.45586 & 2.54947 \\
\hline ! ATOM NAME $=$ 'H_681' & $R=$ & -0.60880 & 0.90986 & 3.08653 \\
\hline ! ATOM NAME $=$ 'H_682' & $R=$ & -0.98414 & 0.96859 & 3.30420 \\
\hline ATOM NAME $=10 \_68$ ' & $R=$ & 0.88949 & 0.92298 & 3.05986 \\
\hline
\end{tabular}

\section{B.5 Fourth Proton-Electron Transfer}

In the following the optimized atomic positions which are used in Fig. 4.25b are provided in cartesian coordinates.

\begin{tabular}{|c|c|c|c|c|c|}
\hline ! LATTICE & $\mathrm{T}=$ & $\begin{array}{l}1.00000 \\
0.00000 \\
1.00000\end{array}$ & $\begin{array}{r}-1.00000 \\
0.00000 \\
1.00000\end{array}$ & $\begin{array}{r}0.00000 \\
10.00000 \\
0.00000\end{array}$ & !END \\
\hline ! ATOM NAME $=$ 'H_011' & $R=$ & 0.12597 & 1.13791 & -1.68710 & !END \\
\hline ! ATOM NAME= 'O_01' & $R=$ & -0.06628 & 1.02834 & -1.53259 & !END \\
\hline ! ATOM NAME $=$ 'H_021' & $R=$ & -0.02520 & 0.10877 & -2.73071 & !END \\
\hline ! ATOM NAME $=$ 'H_022' & $R=$ & 0.39215 & -0.37223 & -1.98772 & !END \\
\hline ! ATOM NAME $=$ '0_02' & $R=$ & 0.08965 & 0.09979 & -1.64902 & !END \\
\hline ! ATOM NAME $=$ 'H_031' & $R=$ & 0.66725 & 1.55377 & -1.11013 & !END \\
\hline ! ATOM NAME= 'O_03' & $\mathrm{R}=$ & 0.19944 & 0.40009 & -1.75532 & !END \\
\hline ! ATOM NAME $=$ 'H_042' & $R=$ & -0.43498 & 0.34032 & -2.24090 & !END \\
\hline ! ATOM NAME= 'O_04' & $R=$ & -0.66533 & 0.39603 & -2.10841 & !END \\
\hline ! ATOM NAME $=$ 'H_051' & $R=$ & -0.26343 & 1.02089 & -2.84869 & !END \\
\hline ! ATOM NAME $=$ 'H_052' & $R=$ & -0.19877 & 0.60854 & -2.84417 & !END \\
\hline ! ATOM NAME $=$ '0_05' & $R=$ & -0.28742 & 0.80453 & -3.01262 & !END \\
\hline ! ATOM NAME $=$ 'H_061' & $R=$ & -0.19990 & -0.44986 & -2.75014 & !END \\
\hline ! ATOM NAME $=$ 'H_062' & $R=$ & -0.08175 & -0.60451 & -2.37499 & !END \\
\hline ! ATOM NAME $=$ 'O_06' & $R=$ & -0.28821 & -0.61314 & -2.55177 & !END \\
\hline ! ATOM NAME $=$ 'H_071' & $R=$ & 0.22362 & 0.36048 & -2.49572 & !END \\
\hline ! ATOM NAME $=$ 'H_072' & $R=$ & -0.05733 & -0.12330 & -3.31541 & !END \\
\hline ! ATOM NAME $=$ '0_07' & $R=$ & -0.03792 & 0.30305 & -2.53997 & !END \\
\hline ! ATOM NAME $=$ 'H_081' & $R=$ & -0.70219 & 0.78607 & -3.07169 & !END \\
\hline ! ATOM NAME= 'O_08' & $R=$ & -0.98454 & 0.80091 & -3.07134 & !END \\
\hline ! ATOM NAME $={ }^{\prime} \mathrm{CA} 1^{\prime}$ & $R=$ & -0.42466 & 0.46652 & 0.50219 & !END \\
\hline ! ATOM NAME $={ }^{\prime} \mathrm{CA} 2^{\prime}$ & $R=$ & 0.42762 & 0.56953 & -0.53196 & !END \\
\hline ! ATOM NAME $={ }^{\prime} \mathrm{CA} 3^{\prime}$ & $R=$ & -0.50844 & 0.63792 & -0.50109 & !END \\
\hline ! ATOM NAME $=$ 'CA4 ${ }^{\prime}$ & $R=$ & 0.62357 & 0.53314 & 0.56455 & !ENI \\
\hline
\end{tabular}




\begin{tabular}{|c|c|c|c|c|c|}
\hline ! ATOM NAME $=$ 'MN1' & $R=$ & 0.03631 & 1.05896 & 0.01423 & !END \\
\hline ! ATOM NAME $=$ 'MN2' & $R=$ & 0.03648 & 0.06003 & 0.00472 & !END \\
\hline ! ATOM NAME $=$ 'MN3' & $R=$ & 0.01989 & 1.05884 & 1.06050 & !EN \\
\hline ! ATOM NAME $=$ 'MN4' & $R=$ & 0.06534 & 0.05204 & 1.04117 & !EN \\
\hline ! ATOM NAME= 'MN5' & $R=$ & 0.04988 & 1.11129 & -1.03997 & !E \\
\hline ! ATOM NAME= 'MN6' & $R=$ & -0.03316 & -0.01013 & -1.02886 & \\
\hline ! ATOM NAME $=10_{-}(\mathrm{A}) 1^{\prime}$ & $R=$ & 0.11054 & 0.17916 & 0.52307 & !E \\
\hline ! ATOM NAME $={ }^{\prime} 0_{-}(\mathrm{A}) 2^{\prime}$ & $R=$ & 0.16001 & 1.11455 & -0.50472 & \\
\hline ! ATOM NAME $={ }^{\prime} 0_{-}(\mathrm{A}) 3^{\prime}$ & $R=$ & -1.05366 & 0.91070 & -0.50982 & !El \\
\hline ! ATOM NAME $=10_{-}(\mathrm{A}) 4$ ' & $R=$ & 0.90785 & -0.01206 & 0.52403 & \\
\hline ! ATOM NAME $={ }^{\prime} O_{-}(\mathrm{B}) 1^{\prime}$ & $R=$ & -0.48128 & -0.05525 & 0.12815 & !E \\
\hline ! ATOM NAME $={ }^{\prime} O_{-}(\mathrm{B}) 2^{\prime}$ & $R=$ & -0.06960 & 0.54064 & -0.11936 & \\
\hline ! ATOM NAME $={ }^{\prime} O_{-}(\mathrm{B}) 3^{\prime}$ & $R=$ & -0.47368 & 1.21101 & 1.12904 & !E \\
\hline ! ATOM NAME $=10_{-}$(B) 4 ' & $R=$ & 1.14362 & 0.56997 & 0.94044 & \\
\hline ! ATOM NAME $={ }^{\prime} 0_{-}$(B) 5 ' & $R=$ & -0.46904 & 1.13187 & -0.12705 & !El \\
\hline ! ATOM NAME $={ }^{\prime} 0_{-}(\mathrm{B}) 6^{\prime}$ & $R=$ & 1.11372 & 0.55192 & 0.12782 & !E \\
\hline ! ATOM NAME $={ }^{\prime} O_{-}(\mathrm{B}) 7^{\prime}$ & $R=$ & -0.47075 & -0.01360 & 0.96075 & !E \\
\hline ! ATOM NAME $={ }^{\prime} O_{-}(\mathrm{B}) 8^{\prime}$ & $R=$ & -0.11753 & 0.56146 & 1.14613 & !El \\
\hline ! ATOM NAME $=10_{-}$(B) 9 ' & $R=$ & -0.45300 & 1.12266 & -0.93213 & $! E !$ \\
\hline ! ATOM NAME $=' 0_{-}$(B) $10^{\prime}$ & $R=$ & 1.22422 & 0.56133 & -1.12889 & $! E !$ \\
\hline ! ATOM NAME $=' 0_{-}$(B) $11^{\prime}$ & $R=$ & -0.48924 & -0.13105 & -1.13449 & \\
\hline ! ATOM NAME $={ }^{\prime} 0_{-}$(B) 12 ' & $R=$ & -0.10105 & 0.49939 & -0.97057 & !E! \\
\hline ! ATOM NAME $=$ 'H_611' & $R=$ & 0.33390 & 1.08126 & 1.62398 & $! \mathrm{El}$ \\
\hline ! ATOM NAME $={ }^{\prime} 0 \_61 '$ & $R=$ & 0.07861 & 1.13306 & 1.56200 & !E! \\
\hline ! ATOM NAME $=$ 'H_621' & $R=$ & 0.17891 & 0.17349 & 2.79298 & !E! \\
\hline ! ATOM NAME $=$ 'H_622' & $R=$ & 0.55420 & -0.31838 & 2.10514 & !EI \\
\hline ! ATOM NAME $=$ '0_62' & $R=$ & 0.02263 & -0.06177 & 1.61351 & !EI \\
\hline ! ATOM NAME $=$ 'H_631' & $R=$ & 0.61922 & 1.55532 & 1.20070 & !E! \\
\hline ! ATOM NAME $=$ 'O_63' & $R=$ & 0.17393 & 0.15798 & 1.82035 & !E! \\
\hline ! ATOM NAME $=$ 'H_642' & $R=$ & -0.24021 & 0.41643 & 2.35431 & !EN \\
\hline ! ATOM NAME = '0_64' & $R=$ & -0.47741 & 0.44515 & 2.22524 & !EN \\
\hline ! ATOM NAME $=$ 'H_651' & $R=$ & -0.05593 & 1.06614 & 2.97050 & !EN \\
\hline ! ATOM NAME $=$ 'H_652' & $R=$ & 0.00610 & 0.65800 & 2.94961 & !E! \\
\hline ! ATOM NAME $=$ '0_65' & $R=$ & -0.08206 & 0.84456 & 3.12776 & !EN \\
\hline ! ATOM NAME $=$ 'H_661' & $R=$ & -0.00293 & -0.40467 & 2.84709 & !E! \\
\hline ! ATOM NAME $=$ 'H_662' & $R=$ & 0.12343 & -0.57921 & 2.48437 & !E! \\
\hline ! ATOM NAME $=$ 'O_66' & $R=$ & -0.08517 & -0.58488 & 2.66095 & !EN \\
\hline ! ATOM NAME $=$ 'H_671' & $R=$ & 0.43338 & 0.42877 & 2.57409 & !EN \\
\hline ! ATOM NAME $=$ 'H_672' & $R=$ & 0.13354 & -0.03431 & 3.38018 & !EN \\
\hline ! ATOM NAME= '0_67' & $R=$ & 0.16913 & 0.37619 & 2.61091 & !EN \\
\hline ! ATOM NAME $=$ 'H_681' & $R=$ & -0.50635 & 0.84469 & 3.16062 & !EN \\
\hline ! ATOM NAME = '0_68' & $R=$ & -0.78634 & 0.87348 & 3.14403 & !EI \\
\hline
\end{tabular}




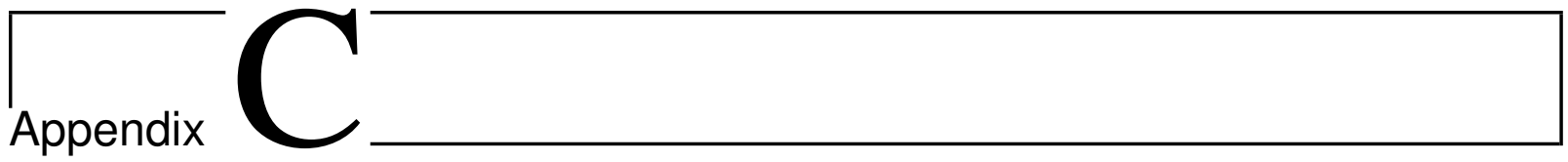

\section{Variable energy positron annihilation spectroscopy (VEPAS)}

For characterization of defect depth profile in $\mathrm{Pd} /$ rutile- $\mathrm{TiO}_{2}$ samples variable energy positron annihilation spectroscopy (VEPAS) [221] was applied. The VEPAS measurements are performed based on a magnetically guided slow positron beam [222]. The energy of incident positrons is set in the range of $80 \mathrm{eV}$ to $35 \mathrm{keV}$. Doppler broadening of the annihilation photopeak is characterized with the sharpness parameter $S$ [223] and measured by a HPGe detector in the energy resolution of $1.09 \mathrm{keV}$ to $511 \mathrm{keV}$. At low incident energies $(E<1 \mathrm{keV})$ positrons annihilate on the sample surface. In the higher energy, positrons penetrate into the $\mathrm{Pd}$ over-layer. In this case, the positrons diffusion back to the surface reduces. At high energy of the incident positrons $(E>13 \mathrm{keV})$ penetration of positrons into the $\mathrm{r}-\mathrm{TiO}_{2}$ observed. For $E>30 \mathrm{keV}$ all positrons are annihilated in rutile- $\mathrm{TiO}_{2}$ and $S$ reaches to the bulk value. No changes were observed for $S$ value at the $\mathrm{Pd} / \mathrm{TiO}_{2}$ interface in a positron energy of $E \approx 13 \mathrm{keV}$. This means hydrogen loading did not introduce any defects of positron trapping, neither into the $\mathrm{r}-\mathrm{TiO}_{2}$ bulk nor into the $\mathrm{Pd} / \mathrm{r}-\mathrm{TiO}_{2}$ interface. It should be noted that the neutral or negative charge defects can trap positron while, the positive charge defects repel positron and VEPAS is not able to observe it.

The VEPAS results are shown in Fig. C.1. we can conclude that hydrogen loading can not introduce $\mathrm{Ti}$ vacancies, while oxygen vacancies can be made. The $S$ parameter have been changed in the $\mathrm{Pd}$ over-layer in the energy range of $\mathrm{E}<13 \mathrm{keV}$. 


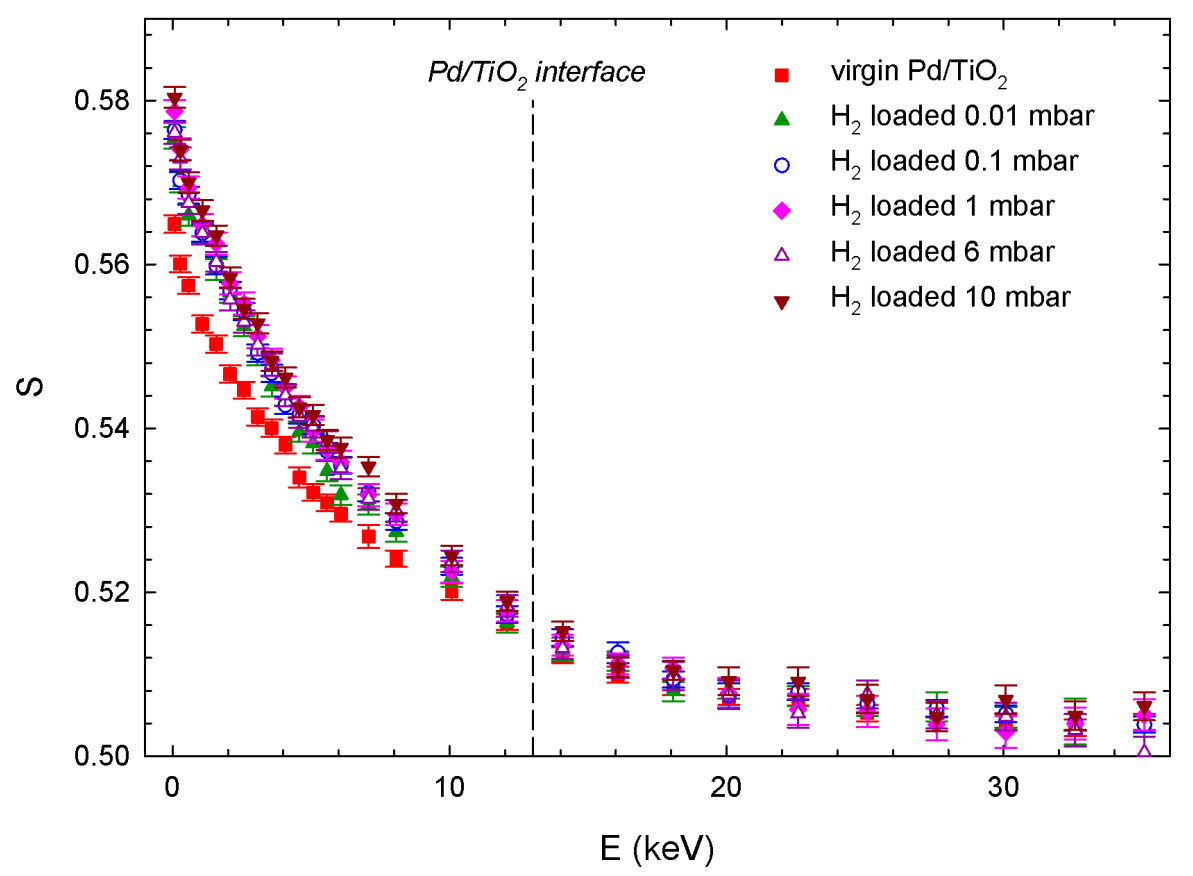

Fig. C.1: VEPAS on the $\mathrm{Pd} /$ rutile- $\mathrm{TiO}_{2}$ system. The $S$ parameter shows sharpness and the $E$ is the positions incident energy. At the interface, there is no changes of the $S$ parameter. With kind approval of J. Cížek. 


\section{Water formation by a hydrogenated oxygen vacancy}

\section{D.0.1 Introduction}

Here we try to derive equations and discuss them in more details.

$$
\frac{3}{2} \mathrm{H}_{2} \leftrightarrow \mathrm{H}_{\mathrm{O}}^{+}+\mathrm{H}_{2} \mathrm{O}+e^{-}
$$

Let us go through the Gibbs free energy:

$$
\begin{aligned}
& \Delta G=G\left[\mathrm{H}_{2} \mathrm{O}\right]-\frac{3}{2} G\left[\mathrm{H}_{2}\right]+E\left[\mathrm{H}_{\mathrm{O}}^{+}\right]-v_{e}\left(\vec{r}_{l}\right)+\mu_{e} \\
& =\quad G\left[\mathrm{H}_{2} \mathrm{O}\right]-\frac{3}{2} G\left[\mathrm{H}_{2}\right]+E\left[\mathrm{H}_{\mathrm{O}}^{+}\right]-v_{e}\left(\vec{r}_{l}\right)+\epsilon_{c}+v_{e}\left(\vec{r}_{l}\right)-\Phi_{B n} \\
& =G\left[\mathrm{H}_{2} \mathrm{O}\right]-\frac{3}{2} G\left[\mathrm{H}_{2}\right]+E\left[\mathrm{H}_{\mathrm{O}}^{+}\right]+\epsilon_{c}-\Phi_{B n} \\
& \text { Equ. D.3, D.4 } E\left[\mathrm{H}_{2} \mathrm{O}\right]-\frac{3}{2} E\left[\mathrm{H}_{2}\right]+E\left[\mathrm{H}_{\mathrm{O}}^{+}\right]+\epsilon_{c}-\Phi_{B n} \\
& +k_{B} T \ln \left(\frac{\left(\lambda_{T}\left[\mathrm{H}_{2} \mathrm{O}\right]\right)^{3} p\left[\mathrm{H}_{2} \mathrm{O}\right]}{k_{B} T}\right)-\frac{3}{2} k_{B} T \ln \left(\frac{\left(\lambda_{T}\left[\mathrm{H}_{2}\right]\right)^{3} p\left[\mathrm{H}_{2}\right]}{k_{B} T}\right) \\
& -k_{B} T \ln \left(g\left[\mathrm{H}_{2} \mathrm{O}\right]\right)+\frac{3}{2} k_{B} T \ln \left(g\left[\mathrm{H}_{2}\right]\right) \\
& -E_{\mathrm{vib}}\left[\mathrm{H}_{2} \mathrm{O}\right]+\frac{3}{2} E_{\mathrm{vib}}\left[\mathrm{H}_{2}\right]-E_{\mathrm{rot}}\left[\mathrm{H}_{2} \mathrm{O}\right]+\frac{3}{2} E_{\mathrm{rot}}\left[\mathrm{H}_{2}\right]
\end{aligned}
$$




$$
\begin{aligned}
& \Delta G=E\left[\mathrm{H}_{2} \mathrm{O}\right]-\frac{1}{2} E\left[\mathrm{H}_{2}\right]-E\left[\mathrm{H}_{2}\right]+E\left[\mathrm{H}_{\mathrm{O}}^{+}\right]+\epsilon_{v}-\epsilon_{v}+\epsilon_{c}-\Phi_{B n} \\
& +\frac{1}{2} E\left[\mathrm{O}_{2}\right]-\frac{1}{2} E\left[\mathrm{O}_{2}\right] \\
& +k_{B} T \ln \left(\frac{\left(\lambda_{T}\left[\mathrm{H}_{2} \mathrm{O}\right]\right)^{3} p\left[\mathrm{H}_{2} \mathrm{O}\right]}{k_{B} T}\right)-\frac{3}{2} k_{B} T \ln \left(\frac{\left(\lambda_{T}\left[\mathrm{H}_{2}\right]\right)^{3} p\left[\mathrm{H}_{2}\right]}{k_{B} T}\right) \\
& +\Delta E_{\mathrm{vib}}+\Delta E_{\mathrm{rot}} \\
& =\underbrace{E\left[\mathrm{H}_{2} \mathrm{O}\right]-E\left[\mathrm{H}_{2}\right]-\frac{1}{2} E\left[\mathrm{O}_{2}\right]}_{-2.554 \mathrm{eV}}+\underbrace{E\left[\mathrm{H}_{\mathrm{O}}^{+}\right]+\epsilon_{v}+\frac{1}{2} E\left[\mathrm{O}_{2}\right]-\frac{1}{2} E\left[\mathrm{H}_{2}\right]}_{1.906 \mathrm{eV}} \\
& +\underbrace{\epsilon_{c}-\epsilon_{v}}_{1.905 \mathrm{eV}}-\underbrace{\Phi_{B n}}_{0.79 \mathrm{eV}}+\underbrace{\Delta E_{\mathrm{vib}}}_{-0.170 \mathrm{eV}}+\underbrace{\Delta E_{\mathrm{rot}}}_{\approx 0 \mathrm{eV}} \\
& +k_{B} T \ln \left(\frac{\left(\lambda_{T}\left[\mathrm{H}_{2} \mathrm{O}\right]\right)^{3} p\left[\mathrm{H}_{2} \mathrm{O}\right]}{k_{B} T}\right) \underbrace{-\frac{3}{2} k_{B} T \ln \left(\frac{\left(\lambda_{T}\left[\mathrm{H}_{2}\right]\right)^{3} p\left[\mathrm{H}_{2}\right]}{k_{B} T}\right)}_{0.804 \mathrm{eV} \mathrm{@} 10 \mathrm{~Pa} \text { of } \mathrm{H}_{2}} \\
& =1.101 \mathrm{eV}+k_{B} T \ln \left(\frac{\left(\lambda_{T}\left[\mathrm{H}_{2} \mathrm{O}\right]\right)^{3} p\left[\mathrm{H}_{2} \mathrm{O}\right]}{k_{B} T}\right)
\end{aligned}
$$

For $\Delta G=0$ (and $p\left[\mathrm{H}_{2}\right]=10 \mathrm{~Pa}$ ) the resulting partial water pressure is about $7.342 \cdot 10^{-8} \mathrm{~Pa}$. We know that with PBE we underestimate reaction energies by about $0.3 \mathrm{eV}$. Adding this to the $1.101 \mathrm{eV}$ results in $1.401 \mathrm{eV}$, and, hence, with $\Delta G=0$, results in a water partial pressure of about $6.23 \cdot 10^{-13} \mathrm{~Pa}$.

\section{D.0.2 Limit of the formation enthalpy for water from rutile tita- nium dioxide}

To estimate limit for the energy of the corrosion reaction we consider the next lower stoichiometric titanium oxide phase which has available data for its formation enthalpies $\Delta H_{f}$. Based on the NIST database this is $\mathrm{Ti}_{3} \mathrm{O}_{5}$ and $\mathrm{Ti}_{2} \mathrm{O}_{3}$. Its and other formation enthalpies are given in tab. D.1. In addition, the formation energy of the molecule $X$ is approximated by the substration of the translational contribution or the $p V$ term, respectively:

$$
\Delta E_{f}[X] \approx \Delta H_{f}[X]+k_{\mathrm{B}} T \ln \left(\frac{\left(\lambda_{T}[X]\right)^{3} \cdot p[X]}{k_{\mathrm{B}} T}\right)
$$

We test the energy of the oxygen molecule by calculating the energy difference for the different titanium oxides: 
Table D.1: Formation enthalpies $\Delta H_{f}$ from experiments are shown. From the enthalpies the corresponding $p V$ term (translational contribution), see Chap. D.0.3, was added to obtain a corresponding estimate of the formation energy $\Delta E_{f}$. Calculated formation energies $\Delta E_{f}^{\mathrm{PBE}}$ obtained with the PBE functional are given for comparison. Experimental enthalpies were taken from [224].

\begin{tabular}{|c|c|c|c|c|c|c|c|c|c|}
\hline Species & $\begin{array}{c}\Delta H_{f} \\
/ \mathrm{kJ} \mathrm{mol}^{-1}\end{array}$ & $\begin{array}{l}\Delta H_{f} \\
/ \mathrm{eV}\end{array}$ & $\begin{array}{c}E_{\text {trans }} \\
/ \mathrm{eV}\end{array}$ & $\begin{array}{l}E_{\text {vib }} \\
/ \mathrm{eV}\end{array}$ & $\begin{array}{l}\Delta E_{f} \\
/ \mathrm{eV}\end{array}$ & $\begin{array}{c}\Delta E_{f}^{\mathrm{PBE}} \\
/ \mathrm{eV}\end{array}$ & $\begin{array}{c}\Delta E_{f}^{\mathrm{PBE}}+E_{\mathrm{vib}} \\
/ \mathrm{eV}\end{array}$ & $\begin{array}{c}\Delta E_{f}^{\mathrm{PBEOr}} \\
/ \mathrm{eV}\end{array}$ & $\begin{array}{c}\Delta E_{f}^{\text {PBE0r }}+E_{\text {vib }} \\
/ \mathrm{eV}\end{array}$ \\
\hline $\mathrm{H}_{2} \mathrm{O}(\mathrm{g})$ & -241.826 & -2.506 & 0.384 & 0.557 & -2.890 & -2.554 & -1.997 & -2.029 & -1.472 \\
\hline $\mathrm{H}_{2}(\mathrm{ref})$ & 0 & 0 & 0.299 & 0.258 & -0.299 & 0 & 0.258 & 0 & 0.258 \\
\hline $\mathrm{O}_{2}(\mathrm{ref})$ & 0 & 0 & 0.406 & 0.098 & -0.406 & 0 & 0.098 & 0 & 0.098 \\
\hline $\mathrm{Ti}(\mathrm{ref})$ & 0 & 0 & 0 & - & 0 & 0 & 0 & 0 & 0 \\
\hline $\mathrm{r}-\mathrm{TiO}_{2}(\mathrm{cr})$ & -944.747 & -9.792 & 0 & - & -9.792 & -8.556 & -8.556 & -8.646 & -8.646 \\
\hline $\mathrm{Ti}_{3} \mathrm{O}_{5}(\mathrm{cr})$ & -2459.146 & -25.487 & 0 & - & -25.487 & -22.709 & -22.709 & & \\
\hline $\mathrm{Ti}_{2} \mathrm{O}_{3}(\mathrm{cr})$ & -1520.844 & -15.762 & 0 & - & -15.762 & -13.922 & -13.922 & & \\
\hline
\end{tabular}

Table D.2: Comparison of the reaction energies between experiment and PBE calculations.

\begin{tabular}{c|c|c|c|c|c}
\hline \hline Reaction & $\begin{array}{c}\Delta E^{\text {exp }} \\
/ \mathrm{eV}\end{array}$ & $\begin{array}{c}\Delta E^{\text {PBE }} \\
/ \mathrm{eV}\end{array}$ & $\begin{array}{c}\text { Difference } \\
/ \mathrm{eV}\end{array}$ & $\begin{array}{c}\Delta E^{\text {PBE0r }} \\
/ \mathrm{eV}\end{array}$ & $\begin{array}{c}\text { Difference } \\
/ \mathrm{eV}\end{array}$ \\
\hline $2 / 3 \mathrm{Ti}_{2} \mathrm{O}_{3} \leftrightarrow 4 / 3 \mathrm{Ti}+\mathrm{O}_{2}$ & 10.103 & 9.379 & 0.723 & 9.471 & 0.631 \\
\hline $4 \mathrm{TiO}_{2} \leftrightarrow 2 \mathrm{Ti}_{2} \mathrm{O}_{3}+\mathrm{O}_{2}$ & 7.236 & 6.476 & 0.760 & 6.564 & 0.673 \\
\hline $6 \mathrm{TiO}_{2} \leftrightarrow 2 \mathrm{Ti}_{3} \mathrm{O}_{5}+\mathrm{O}_{2}$ & 7.369 & 6.013 & 1.356 & 6.350 & 1.020 \\
\hline $4 \mathrm{Ti}_{3} \mathrm{O}_{5} \leftrightarrow 6 \mathrm{Ti}_{2} \mathrm{O}_{3}+\mathrm{O}_{2}$ & 6.969 & 7.401 & -0.432 & 7.857 & -0.021 \\
\hline $2 \mathrm{H}_{2} \mathrm{O} \leftrightarrow 2 \mathrm{H}_{2}+\mathrm{O}_{2}$ & 4.776 & 4.608 & 0.168 & 3.559 & 1.217 \\
\hline \hline $1 / 2 \mathrm{TiO}_{2}+\mathrm{H}_{2} \leftrightarrow 1 / 2 \mathrm{Ti}+\mathrm{H}_{2} \mathrm{O}$ & 2.305 & 2.023 & 0.282 & 2.593 & -0.288 \\
\hline $2 \mathrm{TiO}_{2}+\mathrm{H}_{2} \leftrightarrow \mathrm{Ti}_{2} \mathrm{O}_{3}+\mathrm{H}_{2} \mathrm{O}$ & 1.230 & 0.934 & 0.296 & 1.502 & -0.272 \\
\hline $3 \mathrm{TiO}_{2}+\mathrm{H}_{2} \leftrightarrow \mathrm{Ti}_{3} \mathrm{O}_{5}+\mathrm{H}_{2} \mathrm{O}$ & 1.297 & 0.703 & 0.594 & 1.395 & -0.099 \\
\hline \hline
\end{tabular}




\section{D.0.3 Auxiliary calculations}

\section{Derivation of the molecular Gibbs energies}

$$
\begin{aligned}
& G\left[\mathrm{H}_{2} \mathrm{O}\right]=E\left[\mathrm{H}_{2} \mathrm{O}\right]-k_{B} T \ln \left(g\left[\mathrm{H}_{2} \mathrm{O}\right]\right) \\
& +\quad k_{B} T \ln \left(\frac{\left(\lambda_{T}\left[\mathrm{H}_{2} \mathrm{O}\right]\right)^{3} p\left[\mathrm{H}_{2} \mathrm{O}\right]}{k_{B} T}\right) \\
& -\quad k_{B} T \ln \left(\sum_{n=0}^{\infty} \mathrm{e}^{-\frac{\hbar \omega\left[\mathrm{H}_{2} \mathrm{O}\right]}{k_{B} T}\left(n+\frac{1}{2}\right)}\right) \\
& -\quad k_{B} T \ln \left(\sum_{\ell, m} \mathrm{e}^{-\frac{\hbar^{2} \ell(\ell+1)}{\left[\left[\mathrm{H}_{2} \mathrm{O}\right] k_{B} T\right.}}\right) \\
& =: E\left[\mathrm{H}_{2} \mathrm{O}\right]-k_{B} T \ln \left(g\left[\mathrm{H}_{2} \mathrm{O}\right]\right) \\
& +\quad k_{B} T \ln \left(\frac{\left(\lambda_{T}\left[\mathrm{H}_{2} \mathrm{O}\right]\right)^{3} p\left[\mathrm{H}_{2} \mathrm{O}\right]}{k_{B} T}\right) \\
& \text { - } E_{\text {vib }}\left[\mathrm{H}_{2} \mathrm{O}\right]-E_{\text {rot }}\left[\mathrm{H}_{2} \mathrm{O}\right] \\
& G\left[\mathrm{H}_{2}\right]=E\left[\mathrm{H}_{2}\right]-k_{B} T \ln \left(g\left[\mathrm{H}_{2}\right]\right) \\
& +\quad k_{B} T \ln \left(\frac{\left(\lambda_{T}\left[\mathrm{H}_{2}\right]\right)^{3} p\left[\mathrm{H}_{2}\right]}{k_{B} T}\right) \\
& -\quad k_{B} T \ln \left(\sum_{n=0}^{\infty} \mathrm{e}^{-\frac{\hbar \omega\left[\mathrm{H}_{2}\right]}{k_{B} T}\left(n+\frac{1}{2}\right)}\right) \\
& -\quad k_{B} T \ln \left(\sum_{\ell, m} \mathrm{e}^{-\frac{\hbar^{2} \ell(\ell+1)}{I\left[\mathrm{H}_{2}\right] k_{B} T}}\right) \\
& =: E\left[\mathrm{H}_{2}\right]-k_{B} T \ln \left(g\left[\mathrm{H}_{2}\right]\right) \\
& +k_{B} T \ln \left(\frac{\left(\lambda_{T}\left[\mathrm{H}_{2}\right]\right)^{3} p\left[\mathrm{H}_{2}\right]}{k_{B} T}\right) \\
& \text { - } E_{\mathrm{vib}}\left[\mathrm{H}_{2}\right]-E_{\mathrm{rot}}\left[\mathrm{H}_{2}\right]
\end{aligned}
$$

\section{Ttranslational energy ( $p V$ terms)}

The translational energy or $p V$-term is calculated for the water, hydrogen and oxygen in the following. It does not exist for solids and therefore for $\mathrm{Ti}$ and its oxides. 
The translational contribution for the water molecule at $p=0.1 \mathrm{MPa}$ is:

$$
\begin{aligned}
E_{\text {trans }}\left[\mathrm{H}_{2} \mathrm{O}\right](T=298.15 \mathrm{~K}, p=0.1 \mathrm{MPa}) & =-k_{\mathrm{B}} T \ln \left(\frac{\lambda_{T}^{3} \cdot p}{k_{\mathrm{B}} T}\right) \\
& =-k_{\mathrm{B}} T \ln \left(\frac{\left(2.382 \cdot 10^{-11} / \mathrm{m}\right)^{3} \cdot p}{k_{\mathrm{B}} T}\right) \\
& =0.383567351116381 \mathrm{eV}
\end{aligned}
$$

The translational contribution for the hydrogen molecule at $p=0.1 \mathrm{MPa}$ is:

$$
\begin{aligned}
E_{\text {trans }}\left[\mathrm{H}_{2}\right](T=298.15 \mathrm{~K}, p=0.1 \mathrm{MPa}) & =-k_{\mathrm{B}} T \ln \left(\frac{\lambda_{T}^{3} \cdot p}{k_{\mathrm{B}} T}\right) \\
& =-k_{\mathrm{B}} T \ln \left(\frac{\left(7.121 \cdot 10^{-11} / \mathrm{m}\right)^{3} \cdot p}{k_{\mathrm{B}} T}\right) \\
& =0.29916087638704 \mathrm{eV}
\end{aligned}
$$

The translational contribution for the oxygen molecule at $p=0.1 \mathrm{MPa}$ is:

$$
\begin{aligned}
E_{\text {trans }}\left[\mathrm{O}_{2}\right](T=298.15 \mathrm{~K}, p=0.1 \mathrm{MPa}) & =-k_{\mathrm{B}} T \ln \left(\frac{\lambda_{T}^{3} \cdot p}{k_{\mathrm{B}} T}\right) \\
& =-k_{\mathrm{B}} T \ln \left(\frac{\left(1.787 \cdot 10^{-11} / \mathrm{m}\right)^{3} \cdot p}{k_{\mathrm{B}} T}\right) \\
& =0.405707037467427 \mathrm{eV}
\end{aligned}
$$

\section{Vibrational energy}

Here we calculate the vibrational energy terms for the $\mathrm{H}_{2} \mathrm{O}, \mathrm{H}_{2}$ and $\mathrm{O}_{2}$ molecule. We consider standard conditions which defines the temperature $T=298.15 \mathrm{~K}$.

Using the bending frequencies of water with $f_{1}=c \cdot \tilde{\nu}=c \cdot \frac{1588}{\mathrm{~cm}}=\frac{47607042330400}{\mathrm{~s}}$, $f_{2}=c \cdot \frac{3650}{\mathrm{~cm}}=\frac{109424247170000}{\mathrm{~s}}$ and $f_{3}=c \cdot \frac{3742}{\mathrm{~cm}}=\frac{112182337783600}{\mathrm{~s}}$ [225] the vibrational energy is calculated as

$$
\begin{aligned}
E_{\mathrm{vib}}\left[\mathrm{H}_{2} \mathrm{O}\right]= & -k_{B} T \ln \left(\sum_{n=0}^{\infty} \mathrm{e}^{-\frac{h f_{1}\left[\mathrm{H}_{2} \mathrm{O}\right]}{k_{B} T}\left(n+\frac{1}{2}\right)}\right)-k_{B} T \ln \left(\sum_{n=0}^{\infty} \mathrm{e}^{-\frac{h f_{2}\left[\mathrm{H}_{2} \mathrm{O}\right]}{k_{B} T}\left(n+\frac{1}{2}\right)}\right) \\
& -k_{B} T \ln \left(\sum_{n=0}^{\infty} \mathrm{e}^{-\frac{h f_{3}\left[\mathrm{H}_{2} \mathrm{O}\right]}{k_{B} T}\left(n+\frac{1}{2}\right)}\right) \\
\approx & 0.557 \mathrm{eV}
\end{aligned}
$$

Using the bending frequency of hydrogen with $f=c \cdot \tilde{\nu}=c \cdot \frac{4163}{\mathrm{~cm}}=\frac{124803600265400}{\mathrm{~s}}$ [225] the vibrational energy is calculated as

$$
\begin{aligned}
E_{\mathrm{vib}}\left[\mathrm{H}_{2}\right] & =-k_{B} T \ln \left(\sum_{n=0}^{\infty} \mathrm{e}^{-\frac{h f\left[\mathrm{H}_{2}\right]}{k_{B} T}\left(n+\frac{1}{2}\right)}\right) \\
& \approx 0.258 \mathrm{eV}
\end{aligned}
$$


Using the bending frequency of oxygen with $f=c \cdot \tilde{\nu}=c \cdot \frac{1580}{\mathrm{~cm}}=\frac{47367208364000}{\mathrm{~s}}[225]$ the vibrational energy is calculated as

$$
\begin{aligned}
E_{\mathrm{vib}}\left[\mathrm{O}_{2}\right] & =-k_{B} T \ln \left(\sum_{n=0}^{\infty} \mathrm{e}^{-\frac{h f\left[\mathrm{O}_{2}\right]}{k_{B} T}\left(n+\frac{1}{2}\right)}\right) \\
& \approx 0.098 \mathrm{eV}
\end{aligned}
$$

For the change in the Gibbs energy of the water formation the following energy is required

$$
\Delta E_{\mathrm{vib}}=-E_{\mathrm{vib}}\left[\mathrm{H}_{2} \mathrm{O}\right]+\frac{3}{2} E_{\mathrm{vib}}\left[\mathrm{H}_{2}\right]=-0.169567311667034 \mathrm{eV}
$$

\section{Rotational energy}

Here we calculate the rotational energy terms for the $\mathrm{H}_{2} \mathrm{O}, \mathrm{H}_{2}$ and $\mathrm{O}_{2}$ molecule. We therefore follow equation F2 of the main manuscript. We consider standard conditions which defines the temperature $T=298.15 \mathrm{~K}$.

The moment of inertia is a tensor for the water molecule. Carl W. David [226] diagonalized this tensor and calculated its corresponding values:

$$
I=\left(\begin{array}{ccc}
I_{11} & 0 & 0 \\
0 & I_{22} & 0 \\
0 & 0 & I_{33}
\end{array}\right)=\left(\begin{array}{ccc}
1.09 \cdot 10-40 & 0 & 0 \\
0 & 1.91 \cdot 10-40 & 0 \\
0 & 0 & 3.00 \cdot 10-40
\end{array}\right) \mathrm{g} \mathrm{cm}^{2}
$$

To estimate the influence of the rotational energy of the water molecule, $E_{\mathrm{rot}}\left[\mathrm{H}_{2} \mathrm{O}\right]$ is calculated from the $I_{i i}$ term which yields the largest contribution, which is the $I_{33}$ term:

$$
\begin{aligned}
E_{\mathrm{rot}}\left[\mathrm{H}_{2} \mathrm{O}\right] & =k_{B} T \ln \left(\sum_{l, m}^{\infty} \mathrm{e}^{-\frac{\hbar^{2} l(l+1)}{I_{33}\left[\mathrm{H}_{2} \mathrm{O}\right] k_{B} T}}\right) \\
& \approx 0.06262 \mathrm{eV}
\end{aligned}
$$

Using the moment of inertia for hydrogen with $I\left[\mathrm{H}_{2}\right]=4.607 \cdot 10^{-48} \mathrm{~kg} \mathrm{~m}^{2}$.

$$
\begin{aligned}
E_{\mathrm{rot}}\left[\mathrm{H}_{2}\right] & =k_{B} T \ln \left(\sum_{l, m}^{\infty} \mathrm{e}^{-\frac{\hbar^{2} l(l+1)}{I\left[\mathrm{H}_{2}\right] k_{B} T}}\right) \\
& \approx 0.019 \mathrm{eV}
\end{aligned}
$$

Using the moment of inertia for oxygen with $I\left[\mathrm{O}_{2}\right]=1.945 \cdot 10^{-46} \mathrm{~kg} \mathrm{~m} \mathrm{~m}^{2}$.

$$
\begin{aligned}
E_{\mathrm{rot}}\left[\mathrm{O}_{2}\right] & =k_{B} T \ln \left(\sum_{l, m}^{\infty} \mathrm{e}^{-\frac{\hbar^{2} l(l+1)}{\left[\left[\mathrm{O}_{2}\right] k_{B} T\right.}}\right) \\
& \approx 0.105 \mathrm{eV}
\end{aligned}
$$


For the change in the Gibbs energy of the water formation the following energy is required

$$
\Delta E_{\mathrm{rot}}=-E_{\mathrm{rot}}\left[\mathrm{H}_{2} \mathrm{O}\right]+\frac{3}{2} E_{\mathrm{rot}}\left[\mathrm{H}_{2}\right]=-0.034 \mathrm{eV}
$$


156 D WATER FORMATION BY A HYDROGENATED OXYGEN VACANCY 


\section{Bibliography}

[1] Myron B. Salamon and Marcelo Jaime. The physics of manganites: Structure and transport. Rev. Mod. Phys., 73:583-628, Aug 2001. doi: 10.1103/RevModPhys.73. 583. URL https://link.aps.org/doi/10.1103/RevModPhys.73.583.

[2] Elbio Dagotto, Takashi Hotta, and Adriana Moreo. Colossal magnetoresistant materials: the key role of phase separation. Physics Reports, 344(1):1 - 153, 2001. ISSN 0370-1573. doi: https://doi.org/10.1016/S0370-1573(00)00121-6. URL http://www.sciencedirect.com/science/article/pii/S0370157300001216.

[3] Elbio Dagotto. Nanoscale Phase Separation and Colossal Magneto-resistance. Springer, 2002.

[4] Z. Jirak, S. Krupicka, Z. simsa, and M Dlouha nd S. Vratislav. Neutron diffraction study of $\mathrm{pr}_{1-x} \mathrm{ca}_{x} \mathrm{mno}_{3}$ perovskites. Journal of Magnetism and Magnetic Materials, 53:153, 1985. ISSN 0304-8853. doi: https://doi.org/10.1016/ 0304-8853(85)90144-1. URL http://www.sciencedirect.com/science/article/ pii/0304885385901441.

[5] Ch. Jooss, L. Wu, T. Beetz, R. F. Klie, M. Beleggia, M. A. Schofield, S. Schramm, J. Hoffmann, and Y. Zhu. Polaron melting and ordering as key mechanisms for colossal resistance effects in manganites. Proceedings of the National Academy of Sciences, 104(34):13597-13602, 2007. ISSN 0027-8424. doi: 10.1073/pnas.0702748104. URL http: //www . pnas . org/content/104/34/13597.

[6] Y. Tomioka, A. Asamitsu, H. Kuwahara, Y. Moritomo, and Y. Tokura. Magneticfield-induced metal-insulator phenomena in $\mathrm{pr}_{1-x} \mathrm{Ca}_{x} \mathrm{Mno}_{3}$ with controlled chargeordering instability. Phys. Rev. B, 53:R1689-R1692, Jan 1996. doi: 10.1103/ PhysRevB.53.R1689. URL https://link.aps.org/doi/10.1103/PhysRevB.53. R1689.

[7] Y. Tokura. Critical features of colossal magnetoresistive manganites. Rep. Prog. Phys., 69(3):797, 2006. doi: 10.1088/0034-4885/69/3/R06. URL http://stacks . iop.org/0034-4885/69/i=3/a=R06. 
[8] Masatoshi Imada, Atsushi Fujimori, and Yoshinori Tokura. Metal-insulator transitions. Rev. Mod. Phys., 70:1039-1263, Oct 1998. doi: 10.1103/RevModPhys.70.1039. URL https://link.aps.org/doi/10.1103/RevModPhys.70.1039.

[9] M. J. Rozenberg, I. H. Inoue, and M. J. Sánchez. Strong electron correlation effects in nonvolatile electronic memory devices. Applied Physics Letters, 88(3):033510, 2006. doi: 10.1063/1.2164917. URL https://doi.org/10.1063/1.2164917.

[10] Marco Molinari, David A. Tompsett, Stephen C. Parker, Feridoon Azough, and Robert Freer. Structural, electronic and thermoelectric behaviour camno $_{3}$ and camno $_{3-\delta}$. J. Mater. Chem. A, 2:14109, 2014. doi: 10.1039/C4TA01514B. URL http://dx.doi.org/10.1039/C4TA01514B.

[11] Joonghoe Dho. Electrode size dependent i-v characteristics and photovoltaic effect in the oxide p-n junctions $\mathrm{pr}_{0.7} \mathrm{Ca}_{0.3} \mathrm{mno}_{3} / \mathrm{nb}: \mathrm{Srtio}_{3}$ and $\mathrm{la}_{0.7} \mathrm{Ca}_{0.3} \mathrm{mno}_{3} / \mathrm{nb}$ : $\mathrm{Srtio}_{3}$. Solid State Communications, 150(45):2243 - 2247, 2010. ISSN 0038-1098. doi: https://doi.org/10.1016/j.ssc.2010.09.033. URL http://www.sciencedirect. com/science/article/pii/S0038109810005612.

[12] Gesine Saucke, Jonas Norpoth, Christian Jooss, Dong Su, and Yimei Zhu. Polaron absorption for photovoltaic energy conversion in a manganite-titanate $p n$ heterojunction. Phys. Rev. B, 85:165315, Apr 2012. doi: 10.1103/PhysRevB.85.165315. URL https://link.aps.org/doi/10.1103/PhysRevB.85.165315.

[13] Stephanie Raabe, Daniel Mierwaldt, Jim Ciston, Matthé Uijttewaal, Helge Stein, Jörg Hoffmann, Yimei Zhu, Peter Blöchl, and Christian Jooss. In situ electrochemical electron microscopy study of oxygen evolution activity of doped manganite perovskites. Advanced Functional Materials, 22(16):3378-3388. doi: 10.1002/adfm. 201103173. URL https://onlinelibrary.wiley.com/doi/abs/10.1002/adfm. 201103173.

[14] Kristina N. Ferreira, Tina M. Iverson, Karim Maghlaoui, James Barber, and So Iwata. Architecture of the photosynthetic oxygen-evolving center. Science, 303(5665):1831-1838, 2004. ISSN 0036-8075. doi: 10.1126/science.1093087. URL http://science. sciencemag. org/content/303/5665/1831.

[15] J. Rossmeisl, Z.-W. Qu, H. Zhu, G.-J. Kroes, and J.K. Nørskov. Electrolysis of water on oxide surfaces. Journal of Electroanalytical Chemistry, 607(1):83 - 89, 2007. ISSN 1572-6657. doi: https://doi.org/10.1016/j.jelechem.2006.11.008. URL http://www.sciencedirect.com/science/article/pii/S0022072806006371.

[16] Isabela C. Man, Hai-Yan Su, Federico Calle-Vallejo, Heine A. Hansen, José I. Martínez, Nilay G. Inoglu, John Kitchin, Thomas F. Jaramillo, Jens K. Nørskov, and Jan Rossmeisl. Universality in oxygen evolution electrocatalysis on oxide surfaces. ChemCatChem, 3(7):1159-1165. doi: 10.1002/cctc.201000397. URL https://onlinelibrary.wiley.com/doi/abs/10.1002/cctc. 201000397. 
[17] Jin Suntivich, Kevin J. May, Hubert A. Gasteiger, John B. Goodenough, and Yang Shao-Horn. A perovskite oxide optimized for oxygen evolution catalysis from molecular orbital principles. Science, 334(6061):1383-1385, 2011. ISSN 0036-8075. doi: 10.1126/science.1212858. URL http://science.sciencemag.org/content/334/ $6061 / 1383$.

[18] Wesley T. Hong, Marcel Risch, Kelsey A. Stoerzinger, Alexis Grimaud, Jin Suntivich, and Yang Shao-Horn. Toward the rational design of non-precious transition metal oxides for oxygen electrocatalysis. Energy Environ. Sci., 8:1404-1427, 2015. doi: 10.1039/C4EE03869J. URL http://dx.doi.org/10.1039/C4EE03869J.

[19] S. Satpathy, Zoran S. Popović, and Filip R. Vukajlović. Electronic structure of the perovskite oxides: la ${ }_{1-x} \mathrm{Ca}_{x} \mathrm{mno}_{3}$. Phys. Rev. Lett., 76:960-963, Feb 1996. doi: 10.1103/PhysRevLett.76.960. URL https://link.aps.org/doi/10.1103/ PhysRevLett.76.960.

[20] Vladimir I. Anisimov, Jan Zaanen, and Ole K. Andersen. Band theory and mott insulators: Hubbard $U$ instead of stoner $I$. Phys. Rev. B, 44:943-954, Jul 1991. doi: 10.1103/PhysRevB.44.943. URL http://Iink.aps.org/doi/10.1103/PhysRevB. 44.943.

[21] Axel D. Becke. A new mixing of hartree-fock and local density-functional theories. The Journal of Chemical Physics, 98(2):1372-1377, 1993. doi: 10.1063/1.464304. URL https://doi.org/10.1063/1.464304.

[22] J. Heyd, G.E. Scuseria, and M. Ernzerhof. Hybrid functionals based on a screened coulomb potential. J. Chem. Phys., 118(18):8207, 2003. doi: doi:10.1063/1. 1564060. URL http://scitation.aip.org/content/aip/journal/jcp/118/18/ 10.1063/1.1564060.

[23] Lars Hedin. New method for calculating the one-particle green's function with application to the electron-gas problem. Phys. Rev., 139:A796-A823, Aug 1965. doi: 10.1103/PhysRev.139.A796. URL http://link.aps.org/doi/10.1103/PhysRev. 139. A796.

[24] C. Franchini, R. Kovacik, M Marsman, S. Sathyanarayana Murthy, J. He, C. Ederer, and G. Kresse. Maximally localized wannier functions in lamno $_{3}$ within pbe $+\mathrm{u}$, hybrid functionals and partially self-consistent gw: an efficient route to construct ab initio tight-binding parameters for $\mathrm{e}_{g}$ perovskites. Journal of Physics: Condensed Matter, 24(23):235602, 2012. URL http://stacks.iop.org/0953-8984/24/i=23/ $\mathrm{a}=235602$.

[25] Roman Kováčik, Sowmya Sathyanarayana Murthy, Carmen E. Quiroga, Claude Ederer, and Cesare Franchini. Combined first-principles and model hamiltonian study of the perovskite series $r \mathrm{MnO}_{3}(r=\mathrm{La}, \mathrm{Pr}, \mathrm{Nd}, \mathrm{Sm}, \mathrm{Eu}$, and Gd). Phys. 
Rev. B, 93:075139, Feb 2016. doi: 10.1103/PhysRevB.93.075139. URL https: //link.aps.org/doi/10.1103/PhysRevB.93.075139.

[26] Cesare Franchini. Hybrid functionals applied to perovskites. Journal of Physics: Condensed Matter, 26(25):253202, 2014. URL http://stacks.iop.org/ $0953-8984 / 26 / i=25 / a=253202$.

[27] Claude Ederer, Chungwei Lin, and Andrew J. Millis. Structural distortions and model hamiltonian parameters: From lsda to a tight-binding description of $\mathrm{LaMno}_{3}$. Phys. Rev. B, 76:155105, Oct 2007. doi: 10.1103/PhysRevB.76.155105. URL http: //link.aps.org/doi/10.1103/PhysRevB.76.155105.

[28] Roman Kováčik and Claude Ederer. Calculation of model hamiltonian parameters for lamno $_{3}$ using maximally localized wannier functions. Phys. Rev. B, 81:245108, Jun 2010. doi: 10.1103/PhysRevB.81.245108. URL http://link.aps.org/doi/10. 1103/PhysRevB.81.245108.

[29] Roman Kováčik and Claude Ederer. Effect of hubbard $u$ on the construction of lowenergy hamiltonians for lamno $_{3}$ via maximally localized wannier functions. Phys. Rev. B, 84:075118, Aug 2011. doi: 10.1103/PhysRevB.84.075118. URL http:// link.aps.org/doi/10.1103/PhysRevB.84.075118.

[30] Jiangang He, Ming-Xing Chen, Xing-Qiu Chen, and Cesare Franchini. Structural transitions and transport-half-metallic ferromagnetism in $\mathrm{lamno}_{3}$ at elevated pressure. Phys. Rev. B, 85:195135, May 2012. doi: 10.1103/PhysRevB.85.195135. URL http://link.aps.org/doi/10.1103/PhysRevB.85.195135.

[31] Jiangang He and Cesare Franchini. Screened hybrid functional applied to $3 d^{0} \rightarrow 3 d^{8}$ transition-metal perovskites $\mathrm{lamo}_{3}(\mathrm{~m}=\mathrm{sc}-\mathrm{cu})$ : Influence of the exchange mixing parameter on the structural, electronic, and magnetic properties. Phys. Rev. B, 86: 235117, Dec 2012. doi: 10.1103/PhysRevB.86.235117. URL http://link.aps.org/ doi/10.1103/PhysRevB.86.235117.

[32] A P Ramirez. Colossal magnetoresistance. Journal of Physics: Condensed Matter, 9 (39):8171, 1997. URL http://stacks.iop.org/0953-8984/9/i=39/a=005.

[33] Kliment I Kugel' and D I Khomskii. The jahn-teller effect and magnetism: transition metal compounds. Soviet Physics Uspekhi, 25(4):231, 1982. URL http://stacks. iop.org/0038-5670/25/i=4/a=R03.

[34] Eva Pavarini and Erik Koch. Origin of jahn-teller distortion and orbital order in lamno ${ }_{3}$. Phys. Rev. Lett., 104:086402, Feb 2010. doi: 10.1103/PhysRevLett.104. 086402. URL https://link.aps.org/doi/10.1103/PhysRevLett.104.086402.

[35] Andreas Flesch, Guoren Zhang, Erik Koch, and Eva Pavarini. Orbital-order melting in rare-earth manganites: Role of superexchange. Phys. Rev. B, 85:035124, Jan 
2012. doi: 10.1103/PhysRevB.85.035124. URL https://link.aps.org/doi/10. 1103/PhysRevB.85.035124.

[36] Jolanta Stankiewicz, Javier Sesé, Joaquín García, Javier Blasco, and Conrado Rillo. Magnetic behavior of $\mathrm{pr}_{1-x} \mathrm{Ca}_{x} \mathrm{mno}_{3}$ in the electric-field-driven insulator-metal transition. Phys. Rev. B, 61:11236-11239, May 2000. doi: 10.1103/PhysRevB.61.11236. URL https://link.aps.org/doi/10.1103/PhysRevB.61.11236.

[37] V. I. Anisimov, I. S. Elfimov, M. A. Korotin, and K. Terakura. Orbital and charge ordering in $\mathrm{pr}_{1-\mathrm{x}} \mathrm{Ca}_{\mathrm{x}} \mathrm{mno}_{3}(\mathrm{x}=0$ and 0.5$)$ from the ab initio calculations. Phys. Rev. B, 55:15494-15499, Jun 1997. doi: 10.1103/PhysRevB.55.15494. URL https:// link.aps.org/doi/10.1103/PhysRevB.55.15494.

[38] Jeroen van den Brink, Giniyat Khaliullin, and Daniel Khomskii. Charge and orbital order in half-doped manganites. Phys. Rev. Lett., 83:5118-5121, Dec 1999. doi: 10.1103/PhysRevLett.83.5118. URL http://link.aps.org/doi/10.1103/ PhysRevLett. 83.5118.

[39] S. Grenier, J. P. Hill, Doon Gibbs, K. J. Thomas, M. v. Zimmermann, C. S. Nelson, V. Kiryukhin, Y. Tokura, Y. Tomioka, D. Casa, T. Gog, and C. Venkataraman. Resonant x-ray diffraction of the magnetoresistant perovskite $\mathrm{pr}_{0.6} \mathrm{Ca}_{0.4} \mathrm{mno}_{3}$. Phys. Rev. B, 69:134419, Apr 2004. doi: 10.1103/PhysRevB.69.134419. URL http:// link.aps.org/doi/10.1103/PhysRevB.69.134419.

[40] A. Daoud-Aladine, J. Rodríguez-Carvajal, L. Pinsard-Gaudart, M. T. FernándezDíaz, and A. Revcolevschi. Zener polaron ordering in half-doped manganites. Phys. Rev. Lett., 89:097205, Aug 2002. doi: 10.1103/PhysRevLett.89.097205. URL https: //link.aps.org/doi/10.1103/PhysRevLett.89.097205.

[41] L. Wu, R. F. Klie, Y. Zhu, and Ch. Jooss. Experimental confirmation of zenerpolaron-type charge and orbital ordering in $\mathrm{pr}_{1-x} \mathrm{ca}_{x} \mathrm{mno}_{3}$. Phys. Rev. B, 76:174210, Nov 2007. doi: 10.1103/PhysRevB.76.174210. URL https://link.aps.org/doi/ 10.1103/PhysRevB.76.174210.

[42] Dmitry V. Efremov, Jeroen van den Brink, and Daniel I. Khomskii. Bond- versus site-centred ordering and possible ferroelectricity in manganites. Nature Materials, 3:853, 2004. URL https://doi .org/10.1038/nmat1236.

[43] Giuseppe Colizzi, Alessio Filippetti, and Vincenzo Fiorentini. Multiferroicity and orbital ordering in $\mathrm{pr}_{0.5} \mathrm{Ca}_{0.5} \mathrm{mno}_{3}$ from first principles. Phys. Rev. B, 82:140101, Oct 2010. doi: 10.1103/PhysRevB.82.140101. URL https://link.aps.org/doi/10. 1103/PhysRevB.82.140101.

[44] Gianluca Giovannetti, Sanjeev Kumar, Jeroen van den Brink, and Silvia Picozzi. Magnetically induced electronic ferroelectricity in half-doped manganites. Phys. Rev. 
Lett., 103:037601, Jul 2009. doi: 10.1103/PhysRevLett.103.037601. URL http: //link.aps.org/doi/10.1103/PhysRevLett.103.037601.

[45] Daniel Mierwaldt, Stephanie Mildner, Rosa Arrigo, Axel Knop-Gericke, Emanuel Franke, Andreas Blumenstein, Jörg Hoffmann, and Christian Jooss. In situ xanes/xps investigation of doped manganese perovskite catalysts. Catalysts, 4(2):129-145, 2014. ISSN 2073-4344. URL http://www. mdpi. com/2073-4344/4/2/129.

[46] S. Mildner, J. Hoffmann, P. E. Blöchl, S. Techert, and C. Jooss. Temperature- and doping-dependent optical absorption in the small-polaron system $\operatorname{Pr}_{1-x} \mathrm{Ca}_{x} \mathrm{Mno}_{3}$. Phys. Rev. B, 92:035145, Jul 2015. doi: 10.1103/PhysRevB.92.035145. URL http: //link.aps.org/doi/10.1103/PhysRevB.92.035145.

[47] M. Born and R. Oppenheimer. Zur quantentheorie der molekeln. Annalen der Physik, 389(20):457-484. doi: 10.1002/andp.19273892002. URL https://onlinelibrary. wiley.com/doi/abs/10.1002/andp.19273892002.

[48] M. Born and K. Huang. Dynamical Theory of Crystal Lattices. Oxford University Press, Oxford, 1954.

[49] Attila Szabo and Neil S. Ostlund. Modern Quantum Chemistry. McGraw-Hill, New York, 1989. ISBN 9780070627390. URL http://store.doverpublications.com/ $0486691861 . \mathrm{html}$.

[50] Pierre Hohenberg and Walter Kohn. Inhomogeneous electron gas. Phys. Rev., 136: B864, 1964. doi: 10.1103/PhysRev.136.B864. URL http://link.aps.org/doi/10. 1103/PhysRev.136.B864.

[51] Walter Kohn and Lu J. Sham. Self-consistent equations including exchange and correlation effects. Phys. Rev., 140:A1133, 1965. doi: 10.1103/PhysRev.140.A1133. URL http://link.aps.org/doi/10.1103/PhysRev.140.A1133.

[52] Mel Levy. Universal variational functionals of electron densities, first order density matrixes and natural spin-orbitals and solution of the v-representability problem. Proc. Nat'l Acad. Sci. USA, 76:6062, 1979. URL http://www. pnas.org/content/ 76/12/6062. abstract.

[53] J Harris and R O Jones. The surface energy of a bounded electron gas. Journal of Physics F: Metal Physics, 4(8):1170, 1974. URL http://stacks.iop.org/ $0305-4608 / 4 / i=8 / a=013$.

[54] D.C. Langreth and J.P. Perdew. The exchange-correlation energy of a metallic surface. Solid State Communications, 17(11):1425 - 1429, 1975. ISSN 0038-1098. doi: https://doi.org/10.1016/0038-1098(75)90618-3. URL http://www . sciencedirect. $\mathrm{com} / \mathrm{science/article/pii/0038109875906183.}$ 
[55] O. Gunnarsson and B. I. Lundqvist. Exchange and correlation in atoms, molecules, and solids by the spin-density-functional formalism. Phys. Rev. B, 13:4274-4298, May 1976. doi: 10.1103/PhysRevB.13.4274. URL https://link.aps.org/doi/10. 1103/PhysRevB.13.4274.

[56] J. P. Perdew and Alex Zunger. Self-interaction correction to density-functional approximations for many-electron systems. Phys. Rev. B, 23:5048-5079, May 1981. doi: 10.1103/PhysRevB.23.5048. URL https://link.aps.org/doi/10.1103/ PhysRevB. 23.5048.

[57] D. M. Ceperley and B. J. Alder. Ground state of the electron gas by a stochastic method. Phys. Rev. Lett., 45:566-569, Aug 1980. doi: 10.1103/PhysRevLett.45.566. URL https://link.aps.org/doi/10.1103/PhysRevLett.45.566.

[58] David C. Langreth and M. J. Mehl. Beyond the local-density approximation in calculations of ground-state electronic properties. Phys. Rev. B, 28:1809-1834, Aug 1983. doi: 10.1103/PhysRevB.28.1809. URL https://link.aps.org/doi/10.1103/ PhysRevB . 28.1809.

[59] John P. Perdew, Kieron Burke, and Matthias Ernzerhof. Generalized gradient approximation made simple. Phys. Rev. Lett., 77:3865-3868, Oct 1996. doi: 10.1103/ PhysRevLett.77.3865. URL https://link.aps.org/doi/10.1103/PhysRevLett. 77.3865 .

[60] Axel D. Becke. Density-functional thermochemistry. iii. the role of exact exchange. The Journal of Chemical Physics, 98(7):5648-5652, 1993. doi: 10.1063/1.464913. URL https://doi.org/10.1063/1.464913.

[61] John P. Perdew, Matthias Ernzerhof, and Kieron Burke. Rationale for mixing exact exchange with density functional approximations. The Journal of Chemical Physics, 105(22):9982-9985, 1996. doi: 10.1063/1.472933. URL https://doi.org/10.1063/ 1.472933 .

[62] P. J. Stephens, F. J. Devlin, C. F. Chabalowski, and M. J. Frisch. Ab initio calculation of vibrational absorption and circular dichroism spectra using density functional force fields. The Journal of Physical Chemistry, 98(45):11623-11627, 1994. doi: 10.1021/j100096a001. URL https://doi.org/10.1021/j100096a001.

[63] Mohsen Sotoudeh, Sangeeta Rajpurohit, Peter Blöchl, Daniel Mierwaldt, Jonas Norpoth, Vladimir Roddatis, Stephanie Mildner, Birte Kressdorf, Benedikt Ifland, and Christian Jooss. Electronic structure of $\mathrm{pr}_{1-x} \mathrm{ca}_{x} \mathrm{mno}_{3}$. Phys. Rev. B, 95:235150, Jun 2017. doi: 10.1103/PhysRevB.95.235150. URL https://link.aps.org/doi/ 10.1103/PhysRevB.95.235150.

[64] Carlo Adamo and Vincenzo Barone. Toward reliable density functional methods without adjustable parameters: The pbe0 model. The Journal of Chemical Physics, 
110(13):6158-6170, 1999. doi: 10.1063/1.478522. URL https://doi.org/10.1063/ 1.478522.

[65] P. Novák, J. Kunescaron, L. Chaput, and W. E. Pickett. Exact exchange for correlated electrons. Phys. Status Solidi B, 243:563, 2006.

[66] Fabien Tran, Peter Blaha, Karlheinz Schwarz, and Pavel Novák. Hybrid exchangecorrelation energy functionals for strongly correlated electrons: Applications to transition-metal monoxides. Phys. Rev. B, 74:155108, Oct 2006. doi: 10.1103/ PhysRevB.74.155108. URL https://link.aps.org/doi/10.1103/PhysRevB.74. 155108.

[67] P. E. Blöchl and C. Först. Node-less atomic wave functions, Pauli repulsion and systematic projector augmentation. arXiv, 1210.5937, 2012. URL http://arxiv. org/abs/1210.5937.

[68] O. K. Andersen and O. Jepsen. Explicit, first-principles tight-binding theory. Phys. Rev. Lett., 53:2571-2574, Dec 1984. doi: 10.1103/PhysRevLett.53.2571. URL https: //link.aps.org/doi/10.1103/PhysRevLett.53.2571.

[69] P. E. Blöchl. Projector augmented-wave method. Phys. Rev. B, 50:17953-17979, Dec 1994. doi: 10.1103/PhysRevB.50.17953. URL http://link.aps.org/doi/10. 1103/PhysRevB.50.17953.

[70] D. R. Hamann, M. Schlüter, and C. Chiang. Norm-conserving pseudopotentials. Phys. Rev. Lett., 43:1494-1497, Nov 1979. doi: 10.1103/PhysRevLett.43.1494. URL https://link.aps.org/doi/10.1103/PhysRevLett.43.1494.

[71] O. Krogh Andersen. Linear methods in band theory. Phys. Rev. B, 12:3060-3083, Oct 1975. doi: 10.1103/PhysRevB.12.3060. URL https://link.aps.org/doi/10. 1103/PhysRevB.12.3060.

[72] Roberto Car and Michele Parrinello. Unified approach for molecular dynamics and density-functional theory. Phys. Rev. Lett, 55:2471, 1985. doi: 10.1103/PhysRevLett. 55.2471. URL http://link.aps.org/doi/10.1103/PhysRevLett.55.2471.

[73] Frederick Wooten. Optical Properties of Solids. Academic Press, 1972.

[74] R.F. Egerton. Limits to the spatial, energy and momentum resolution of electron energy-loss spectroscopy. Ultramicroscopy, 107(8):575 - 586, 2007. ISSN 0304-3991. doi: https://doi.org/10.1016/j.ultramic.2006.11.005. URL http://www . sciencedirect.com/science/article/pii/S0304399106002245.

[75] M.P. Seah and W.A. Dench. Quantitative electron spectroscopy of surfaces: A standard data base for electron inelastic mean free paths in solids. Surf. Interface Anal., 1:2, 1979. URL http://dx.doi.org/10.1002/sia.740010103. 
[76] Robert Laskowski and Peter Blaha. Understanding the $L_{2,3}$ x-ray absorption spectra of early 3d transition elements. Phys. Rev. B, 82:205104, Nov 2010. doi: 10.1103/PhysRevB.82.205104. URL https://link.aps.org/doi/10.1103/ PhysRevB. 82.205104.

[77] I. Davoli, A. Marcelli, A. Bianconi, M. Tomellini, and M. Fanfoni. Multielectron configurations in the x-ray-absorption near-edge structure of nio at the oxygen $\mathrm{k}$ threshold. Phys. Rev. B, 33:2979-2982, Feb 1986. doi: 10.1103/PhysRevB.33.2979. URL https://link.aps.org/doi/10.1103/PhysRevB.33.2979.

[78] G.H. Jonker and J.H. Van Santen. Ferromagnetic compounds of manganese with perovskite structure. Physica, 16(3):337 - 349, 1950. ISSN 0031-8914. doi: http:// dx.doi.org/10.1016/0031-8914(50)90033-4. URL http://www.sciencedirect.com/ science/article/pii/0031891450900334.

[79] J.H. Van Santen and G.H. Jonker. Electrical conductivity of ferromagnetic compounds of manganese with perovskite structure. Physica, 16(7):599 - 600, 1950. ISSN 0031-8914. doi: http://dx.doi.org/10.1016/0031-8914(50)90104-2. URL http: //www.sciencedirect.com/science/article/pii/0031891450901042.

[80] G.H. Jonker and J.H. Van Santen. Magnetic compounds wtth perovskite structure iii. ferromagnetic compounds of cobalt. Physica, 19(1):120 - 130, 1953. ISSN 00318914. doi: http://dx.doi.org/10.1016/S0031-8914(53)80011-X. URL http://www . sciencedirect.com/science/article/pii/s003189145380011X.

[81] E. O. Wollan and W. C. Koehler. Neutron diffraction study of the magnetic properties of the series of perovskite-type compounds $[(1-x) \mathrm{La}, x \mathrm{Ca}] \mathrm{Mno}_{3}$. Phys. Rev., 100: 545-563, Oct 1955. doi: 10.1103/PhysRev.100.545. URL https://link.aps.org/ doi/10.1103/PhysRev.100.545.

[82] Ken ichi Chahara, Toshiyuki Ohno, Masahiro Kasai, and Yuzoo Kozono. Magnetoresistance in magnetic manganese oxide with intrinsic antiferromagnetic spin structure. Applied Physics Letters, 63(14):1990-1992, 1993. doi: 10.1063/1.110624. URL http://dx.doi.org/10.1063/1.110624.

[83] H. L. Ju, C. Kwon, Qi Li, R. L. Greene, and T. Venkatesan. Giant magnetoresistance in la $\mathrm{la}_{1-x} \mathrm{Sr}_{x} \mathrm{mno}_{z}$ films near room temperature. Applied Physics Letters, 65(16):21082110, 1994. doi: 10.1063/1.112808. URL http://dx.doi.org/10.1063/1.112808.

[84] S. Jin, T. H. Tiefel, M. McCormack, R. A. Fastnacht, R. Ramesh, and L. H. Chen. Thousandfold change in resistivity in magnetoresistive la-ca-mn-o films. Science, 264 (5157):413-415, 1994. ISSN 0036-8075. doi: 10.1126/science.264.5157.413. URL http://science.sciencemag. org/content/264/5157/413. 
[85] John B. Goodenough. Theory of the role of covalence in the perovskite-type manganites [La, $m(\mathrm{II}) \mathrm{Mno}_{3}$. Phys. Rev., 100:564-573, Oct 1955. doi: 10.1103/PhysRev. 100.564. URL http://link.aps.org/doi/10.1103/PhysRev.100.564.

[86] N. Mannella, W. L. Yang, X. J. Zhou, H. Zheng, J. F. Mitchell, J. Zaanen, T. P. Devereaux, N. Nagaosa, Z. Hussain, and Z.-X. Shen. Nodal quasiparticle in pseudogapped colossal magnetoresistive manganites. Nature, 438:474, 2005. URL https://doi.org/10.1038/nature04273.

[87] Dirk Raiser, Stephanie Mildner, Benedikt Ifland, Mohsen Sotoudeh, Peter Blöchl, Simone Techert, and Christian Jooss. Evolution of hot polaron states with a nanosecond lifetime in a manganite perovskite. Advanced Energy Materials, 7(12):1602174, 2017. doi: 10.1002/aenm.201602174. URL https://onlinelibrary.wiley.com/ doi/abs/10.1002/aenm. 201602174.

[88] V. M. Goldschmidt. Die gesetze der krystallochemie. Naturwissenschaften, 14:477, 1926. URL https://doi.org/10.1007/BF01507527.

[89] Zhen Li, Mengjin Yang, Ji-Sang Park, Su-Huai Wei, Joseph J. Berry, and Kai Zhu. Stabilizing perovskite structures by tuning tolerance factor: Formation of formamidinium and cesium lead iodide solid-state alloys. Chemistry of Materials, 28(1): 284-292, 2016. doi: 10.1021/acs.chemmater.5b04107. URL https://doi.org/10. 1021 /acs. chemmater. 5b04107.

[90] A. M. Glazer. The classification of tilted octahedra in perovskites. Acta Crystallographica Section B, 28(11):3384-3392, Nov 1972. doi: $10.1 \overline{107 /}$ S0567740872007976. URL https://doi .org/10.1107/S0567740872007976.

[91] Takashi Hotta. Orbital ordering phenomena in d- and f-electron systems. Reports on Progress in Physics, 69(7):2061, 2006. URL http://stacks.iop.org/0034-4885/ $69 / i=7 / a=R 02$.

[92] H. A. Jahn and E. Teller. Stability of polyatomic molecules in degenerate electronic states - i-orbital degeneracy. Proceedings of the Royal Society of London A: Mathematical, Physical and Engineering Sciences, 161(905):220-235, 1937. ISSN 0080-4630. doi: 10.1098/rspa.1937.0142. URL http://rspa. royalsocietypublishing. org/content/161/905/220.

[93] Junjiro Kanamori. Crystal distortion in magnetic compounds. Journal of Applied Physics, 31(5):S14-S23, 1960. doi: 10.1063/1.1984590. URL https://doi.org/10. 1063/1. 1984590.

[94] M. v. Zimmermann, J. P. Hill, Doon Gibbs, M. Blume, D. Casa, B. Keimer, Y. Murakami, Y. Tomioka, and Y. Tokura. Interplay between charge, orbital, and magnetic order in $\mathrm{pr}_{1-x} \mathrm{Ca}_{x} \mathrm{mno}_{3}$. Phys. Rev. Lett., 83:4872-4875, Dec 1999. 
doi: 10.1103/PhysRevLett.83.4872. URL https://link.aps.org/doi/10.1103/ PhysRevLett.83.4872.

[95] Takashi Hotta and Elbio Dagotto. Competition between ferromagnetic and chargeorbital ordered phases in $\mathrm{pr}_{1-x} \mathrm{Ca}_{x} \mathrm{mno}_{3}$ for $x=\frac{1}{4}, \frac{3}{8}$, and $\frac{1}{2}$. Phys. Rev. B, 61: R11879-R11882, May 2000. doi: 10.1103/PhysRevB.61.R11879. URL https:// link.aps.org/doi/10.1103/PhysRevB.61.R11879.

[96] H. Yoshizawa, H. Kawano, Y. Tomioka, and Y. Tokura. Neutron-diffraction study of the magnetic-field-induced metal-insulator transition in $\mathrm{pr}_{0.7} \mathrm{Ca}_{0.3} \mathrm{mno}_{3}$. Phys. Rev. B, 52:R13145-R13148, Nov 1995. doi: 10.1103/PhysRevB.52.R13145. URL https: //link.aps.org/doi/10.1103/PhysRevB.52.R13145.

[97] C. de Graaf, C. Sous, and R. Broer. Ab initio study of the charge order and zener polaron formation in half-doped manganites. Phys. Rev. B, 70:235104, 2004.

[98] G. Zheng and C. H. Patterson. Ferromagnetic polarons in $\mathrm{la}_{0.5} \mathrm{Ca}_{0.5} \mathrm{mno}_{3}$ and $\mathrm{la}_{0.33} \mathrm{Ca}_{0.67} \mathrm{mno}_{3}$. Phys. Rev. B, 67:220404, Jun 2003. doi: 10.1103/PhysRevB.67. 220404. URL https://link.aps.org/doi/10.1103/PhysRevB.67.220404.

[99] C. H. Patterson. Competing crystal structures in $\mathrm{la}_{0.5} \mathrm{ca}_{0.5} \mathrm{Mno}_{3}$ : Conventional charge order versus zener polarons. Phys. Rev. B, 72:085125, Aug 2005. doi: 10.1103/ PhysRevB.72.085125. URL https://link.aps.org/doi/10.1103/PhysRevB.72. 085125.

[100] Giuseppe Colizzi, Alessio Filippetti, and Vincenzo Fiorentini. Multiferroicity and orbital ordering in $\mathrm{pr}_{0.5} \mathrm{Ca}_{0.5} \mathrm{mno}_{3}$ from first principles. Phys. Rev. B, 82:140101, Oct 2010. doi: 10.1103/PhysRevB.82.140101. URL https://link.aps.org/doi/10. 1103/PhysRevB.82.140101.

[101] C. Zener. Interaction between the $d$ shells in the transition metals. Phys. Rev., 81: 440-444, Feb 1951. doi: 10.1103/PhysRev.81.440. URL https://link.aps.org/ doi/10.1103/PhysRev.81.440.

[102] Clarence Zener. Interaction between the $d$-shells in the transition metals. ii. ferromagnetic compounds of manganese with perovskite structure. Phys. Rev., 82:403-405, May 1951. doi: 10.1103/PhysRev.82.403. URL https://link.aps.org/doi/10. 1103/PhysRev.82.403.

[103] P. W. Anderson and H. Hasegawa. Considerations on double exchange. Phys. Rev., 100:675-681, Oct 1955. doi: 10.1103/PhysRev.100.675. URL https://link.aps. org/doi/10.1103/PhysRev.100.675.

[104] J. Rodríguez-Carvajal, M. Hennion, F. Moussa, A. H. Moudden, L. Pinsard, and A. Revcolevschi. Neutron-diffraction study of the jahn-teller transition in stoichiometric lamno . Phys. Rev. B, 57:R3189-R3192, Feb 1998. doi: 10.1103/PhysRevB. 57.R3189. URL https://link.aps.org/doi/10.1103/PhysRevB.57.R3189. 
[105] J. H. Van Vleck. The jahn-teller effect and crystalline stark splitting for clusters of the form xy6. The Journal of Chemical Physics, 7(1):72-84, 1939. doi: 10.1063/1. 1750327. URL https://doi.org/10.1063/1.1750327.

[106] Sangeeta Rajpurohit. The study of magnetic and polaronic microstructure in $\mathrm{Pr}_{1-x} \mathrm{Ca}_{x} \mathrm{MnO}_{3}$ manganite series. PhD thesis, Fakultät für Physik (inkl. GAUSS), Georg-August-Universität Göttingen, 2018. URL http://hdl.handle.net/11858/ 00-1735-0000-002E-E4AD-0.

[107] M. Nic, J. Jirat, and B. Koata, editors. IUPAC Compendium of Chemical Terminology - (the "Gold Book". International Union of Pure and Applied Chemistry, 2014. doi: doi:10.1351/goldbook. URL http://goldbook.iupac.org.

[108] K.R. Poeppelmeier, M.E. Leonowicz, J.C. Scanlon, J.M. Longo, and W.B. Yelon. Structure determination of camno $_{3}$ and camno $_{2.5}$ by x-ray and neutron methods. Journal of Solid State Chemistry, 45(1):71 - 79, 1982. ISSN 0022-4596. doi: https: //doi.org/10.1016/0022-4596(82)90292-4. URL http://www. sciencedirect.com/ science/article/pii/0022459682902924.

[109] N. N. Loshkareva, L. V. Nomerovannaya, E. V. Mostovshchikova, A. A. Makhnev, Yu. P. Sukhorukov, N. I. Solin, T. I. Arbuzova, S. V. Naumov, N. V. Kostromitina, A. M. Balbashov, and L. N. Rybina. Electronic structure and polarons in $\mathrm{CaMno}_{3-\delta}$ single crystals: Optical data. Phys. Rev. B, 70:224406, Dec 2004. doi: 10.1103/PhysRevB.70.224406. URL https://link.aps.org/doi/10.1103/ PhysRevB. 70.224406.

[110] Warren E. Pickett and David J. Singh. Electronic structure and half-metallic transport in the $\mathrm{la}_{1-x} \mathrm{Ca}_{x} \mathrm{mno}_{3}$ system. Phys. Rev. B, 53:1146-1160, Jan 1996. doi: 10. 1103/PhysRevB.53.1146. URL https://link.aps.org/doi/10.1103/PhysRevB. 53.1146.

[111] Shannon R. D. Revised effective ionic radii and systematic studies of interatomic distances in halides and chalcogenides. Acta Crystallographica Section A, 32(5):751767. doi: 10.1107/S0567739476001551. URL https://onlinelibrary.wiley.com/ doi/abs/10.1107/S0567739476001551.

[112] Ibrahim Kurash, Liu Fengqin, Qian Haijie, Guo Lin, Xian Dingchang, Xiong Guangcheng, and Wu Sicheng. Valence band photoemission behavior of pr1ấĽ́xsrxmno3. Materials Science and Engineering: B, 76(1):14 - 17, 2000. ISSN 0921-5107. doi: https://doi.org/10.1016/S0921-5107(00)00391-3. URL http: //www.sciencedirect.com/science/article/pii/S0921510700003913.

[113] J. A. Alonso, M. J. Martínez-Lope, M. T. Casais, and M. T. Fernández-Díaz. Evolution of the jahn-teller distortion of mno6 octahedra in rmno3 perovskites (r) pr, nd, dy, tb, ho, er, y): A neutron diffraction study. Inorganic Chemistry, 39(5):917-923, 
2000. doi: 10.1021/ic990921e. URL https://doi.org/10.1021/ic990921e. PMID: 12526369 .

[114] K. Ebata, M. Hashimoto, K. Tanaka, A. Fujimori, Y. Tomioka, and Y. Tokura. Temperature-dependent photoemission spectra, spectral weight transfer, and chemical potential shift in $\mathrm{pr}_{1-x} \mathrm{Ca}_{x} \mathrm{Mno}_{3}$ : Implications for charge-density modulation. Phys. Rev. B, 76:174418, Nov 2007. doi: 10.1103/PhysRevB.76.174418. URL https://link.aps.org/doi/10.1103/PhysRevB.76.174418.

[115] Y. Okimoto, Y. Tomioka, Y. Onose, Y. Otsuka, and Y. Tokura. Charge ordering and disordering transitions in $\mathrm{pr}_{1-x} \mathrm{Ca}_{x} \mathrm{mnO}_{3}(x=0.4)$ as investigated by optical spectroscopy. Phys. Rev. B, 57:R9377, Apr 1998. doi: 10.1103/PhysRevB.57.R9377. URL http://link.aps.org/doi/10.1103/PhysRevB.57.R9377.

[116] H. Wadati, A. Maniwa, I. Ohkubo, H. Kumigashira, A. Fujimori, M. Oshima, M. Lippmaa, M. Kawasaki, and H. Koinuma. In situ photoemission study of $\mathrm{pr}_{1-x} \mathrm{Ca}_{x} \mathrm{mno}_{3}$ epitaxial thin films. Journal of Magnetism and Magnetic Materials, 310(2, Part 2):963 - 965, 2007. ISSN 0304-8853. doi: https://doi.org/10.1016/ j.jmmm.2006.10.346. URL http://www.sciencedirect.com/science/article/ pii/S0304885306015484. Proceedings of the 17th International Conference on Magnetism.

[117] Ibrahim Kurash, Liu Fengqin, Qian Haijie, Guo Lin, Xian Dingchang, Xiong Guangcheng, and $\mathrm{Wu}$ Sicheng. Valence band photoemission behavior of $\mathrm{pr}_{1-x} \mathrm{Sr}_{x} \mathrm{mno}_{3}$. Materials Science and Engineering: B, 76(1):14 - 17, 2000. ISSN 0921-5107. doi: https://doi.org/10.1016/S0921-5107(00)00391-3. URL http://www . sciencedirect.com/science/article/pii/S0921510700003913.

[118] Weidong Luo, Alberto Franceschetti, Maria Varela, Jing Tao, Stephen J. Pennycook, and Sokrates T. Pantelides. Orbital-occupancy versus charge ordering and the strength of electron correlations in electron-doped camno 3 . Phys. Rev. Lett., 99:036402, Jul 2007. doi: 10.1103/PhysRevLett.99.036402. URL http: //link.aps.org/doi/10.1103/PhysRevLett.99.036402.

[119] J. Hemberger, A. Krimmel, T. Kurz, H.-A. Krug von Nidda, V. Yu. Ivanov, A. A. Mukhin, A. M. Balbashov, and A. Loidl. Structural, magnetic, and electrical properties of single-crystalline $\mathrm{la}_{1-x} \mathrm{Sr}_{x} \mathrm{mno}_{3}(0.4<x<0.85)$. Phys. Rev. B, 66:094410, Sep 2002. doi: 10.1103/PhysRevB.66.094410. URL https://link.aps.org/doi/ 10.1103/PhysRevB.66.094410.

[120] Rune Søndenå, P. Ravindran, Svein Stølen, Tor Grande, and Michael Hanfland. Electronic structure and magnetic properties of cubic and hexagonal $\mathrm{SrMno}_{3}$. Phys. Rev. B, 74:144102, Oct 2006. doi: 10.1103/PhysRevB.74.144102. URL https:// link.aps.org/doi/10.1103/PhysRevB.74.144102. 
[121] P.D. Battle, T.C. Gibb, and C.W. Jones. The structural and magnetic properties of srmno3: A reinvestigation. Journal of Solid State Chemistry, 74(1):60 - 66, 1988. ISSN 0022-4596. doi: https://doi.org/10.1016/0022-4596(88)90331-3. URL http: //www.sciencedirect.com/science/article/pii/0022459688903313.

[122] BjÄ̈̈rn C. Hauback, Helmer FjellvÃěg, and Natsuko Sakai. Effect of nonstoichiometry on properties of la $1-t \mathrm{mno}_{3+\delta}$ : Iii. magnetic order studied by powder neutron diffraction. Journal of Solid State Chemistry, 124(1):43 - 51, 1996. ISSN 0022-4596. doi: https://doi.org/10.1006/jssc.1996.0205. URL http://www.sciencedirect. com/science/article/pii/S0022459696902054.

[123] Michael GrÃd'tzel. Solar energy conversion by dye-sensitized photovoltaic cells. Inorg. Chem., 44(20):6841-6851, 2005. doi: 10.1021/ic0508371. URL https://doi.org/ 10.1021/ic0508371. PMID: 16180840.

[124] Michael G. Walter, Emily L. Warren, James R. McKone, Shannon W. Boettcher, Qixi Mi, Elizabeth A. Santori, and Nathan S. Lewis. Solar water splitting cells. Chemical Reviews, 110(11):6446-6473, 2010. doi: 10.1021/cr1002326. URL https: //doi.org/10.1021/cr1002326. PMID: 21062097.

[125] J. K. Nørskov, J. Rossmeisl, A. Logadottir, L. Lindqvist, J. R. Kitchin, T. Bligaard, and H. J $\widetilde{A} \check{C} \hat{A}$ şnsson. Origin of the overpotential for oxygen reduction at a fuel-cell cathode. The Journal of Physical Chemistry B, 108(46):17886-17892, 2004. doi: 10.1021/jp047349j. URL https://doi.org/10.1021/jp047349j.

[126] Michael G. Mavros, Takashi Tsuchimochi, Tim Kowalczyk, Alexandra McIsaac, LeePing Wang, and Troy Van Voorhis. What can density functional theory tell us about artificial catalytic water splitting? Inorganic Chemistry, 53(13):6386-6397, 2014. doi: 10.1021/ic5002557. URL https://doi.org/10.1021/ic5002557. PMID: 24694041.

[127] Eric McCalla, Artem M. Abakumov, Matthieu Saubanère, Dominique Foix, Erik J. Berg, Gwenaelle Rousse, Marie-Liesse Doublet, Danielle Gonbeau, Petr Novák, Gustaaf Van Tendeloo, Robert Dominko, and Jean-Marie Tarascon. Visualization of o-o peroxo-like dimers in high-capacity layered oxides for li-ion batteries. Science, 350(6267):1516-1521, 2015. ISSN 0036-8075. doi: 10.1126/science.aac8260. URL http://science. sciencemag. org/content/350/6267/1516.

[128] Marcel Risch, Kelsey A. Stoerzinger, Shingo Maruyama, Wesley T. Hong, Ichiro Takeuchi, and Yang Shao-Horn. La0.8sr0.2mno3âLŠ̂̀t decorated with ba0.5sr0.5co0.8fe0.2o3âĹŠ̂te: A bifunctional surface for oxygen electrocatalysis with enhanced stability and activity. Journal of the American Chemical Society, 136 (14):5229-5232, 2014. doi: 10.1021/ja5009954. URL https://doi.org/10.1021/ ja5009954. PMID: 24649849.

[129] Xi Rong, Jules Parolin, and Alexie M. Kolpak. A fundamental relationship between reaction mechanism and stability in metal oxide catalysts for oxygen evolution. ACS 
Catalysis, 6(2):1153-1158, 2016. doi: 10.1021/acscatal.5b02432. URL https://doi. org/10.1021/acscatal.5b02432.

[130] Matthew W. Kanan and Daniel G. Nocera. In situ formation of an oxygen-evolving catalyst in neutral water containing phosphate and co2+. Science, 321(5892):10721075, 2008. ISSN 0036-8075. doi: 10.1126/science.1162018. URL http://science. sciencemag.org/content/321/5892/1072.

[131] J. Tyler Mefford, Xi Rong, Artem M. Abakumov, William G. Hardin, Sheng Dai, Alexie M. Kolpak, Keith P. Johnston, and Keith J. Stevenson. Water electrolysis on $\mathrm{la}_{1-x} \mathrm{Sr}_{x} \mathrm{COO}_{3-\delta}$ perovskite electrocatalysts. Nature Communications, 7:11053 EP -, 03 2016. URL http://dx.doi.org/10.1038/ncomms11053.

[132] Alexis Grimaud, Oscar Diaz-Morales, Binghong Han, Wesley T. Hong, Yueh-Lin Lee, Livia Giordano, Kelsey A. Stoerzinger, Marc T. M. Koper, and Yang ShaoHorn. Activating lattice oxygen redox reactions in metal oxides to catalyse oxygen evolution. Nature Chemistry, 9:457 EP -, 01 2017. URL http://dx.doi.org/10. 1038/nchem. 2695.

[133] Jong Suk Yoo, Yusu Liu, Xi Rong, and Alexie M. Kolpak. Electronic origin and kinetic feasibility of the lattice oxygen participation during the oxygen evolution reaction on perovskites. The Journal of Physical Chemistry Letters, 9(7):1473-1479, 2018. doi: 10.1021/acs.jpclett.8b00154. URL https://doi.org/10.1021/acs.jpclett. 8b00154. PMID: 29510623.

[134] Min Ho Seo, Hey Woong Park, Dong Un Lee, Moon Gyu Park, and Zhongwei Chen. Design of highly active perovskite oxides for oxygen evolution reaction by combining experimental and ab initio studies. ACS Catalysis, 5(7):4337-4344, 2015. doi: 10. 1021/acscatal.5b00114. URL https://doi.org/10.1021/acscatal.5b00114.

[135] P.E. Blöchl. Electrostatic decoupling of periodic images of plane-wave-expanded densities and derived atomic point charges. The Journal of Chemical Physics, 103 (17):7422-7428, 1995. doi: 10.1063/1.470314. URL https://doi.org/10.1063/1. 470314.

[136] J. B. Hasted. Water A comprehensive treatise, volume 1, chapter Liquid water: Dielectric properties, pages 255-309. Plenum Press, New York, 1972.

[137] Biswajit Santra, Angelos Michaelides, and Matthias Scheffler. Coupled cluster benchmarks of water monomers and dimers extracted from density-functional theory liquid water: The importance of monomer deformations. The Journal of Chemical Physics, 131(12):124509, 2009. doi: 10.1063/1.3236840. URL https://doi.org/10.1063/1. 3236840 . 
[138] Kevin Leung and Susan B. Rempe. Ab initio rigid water: Effect on water structure, ion hydration, and thermodynamics. Phys. Chem. Chem. Phys., 8:2153-2162, 2006. doi: 10.1039/B515126K. URL http://dx.doi.org/10.1039/B515126K.

[139] Qianyi Cheng, Francesco A. Evangelista, Andrew C. Simmonett, Yukio Yamaguchi, and Henry F. Schaefer. Water dimer radical cation: Structures, vibrational frequencies, and energetics. The Journal of Physical Chemistry A, 113(49):13779-13789, 2009. doi: 10.1021/jp907715a. URL https://doi.org/10.1021/jp907715a. PMID: 19891464.

[140] Mathias Van Thiel, Edwin D. Becker, and George C. Pimentel. Infrared studies of hydrogen bonding of water by the matrix isolation technique. The Journal of Chemical Physics, 27(2):486-490, 1957. doi: 10.1063/1.1743753. $\overline{\text { URL https:// }}$ doi.org/10.1063/1.1743753.

[141] Anamika Mukhopadhyay, William T.S. Cole, and Richard J. Saykally. The water dimer i: Experimental characterization. Chemical Physics Letters, 633:13 - 26, 2015. ISSN 0009-2614. doi: https://doi.org/10.1016/j.cplett.2015.04.016. URL http:// wWw.sciencedirect.com/science/article/pii/S0009261415002535.

[142] J. Rossmeisl, A. Logadottir, and J.K. Nørskov. Electrolysis of water on (oxidized) metal surfaces. Chemical Physics, 319(1):178 - 184, 2005. ISSN 0301-0104. doi: https: //doi.org/10.1016/j.chemphys.2005.05.038. URL http://www . sciencedirect.com/ science/article/pii/S0301010405002053. Molecular Charge Transfer in Condensed Media - from Physics and Chemistry to Biology and Nanoengineering in honour of Alexander M. Kuznetsov on his 65th birthday.

[143] Ye-Fei Li and Annabella Selloni. Pathway of photocatalytic oxygen evolution on aqueous tio2 anatase and insights into the different activities of anatase and rutile. ACS Catalysis, 6(7):4769-4774, 2016. doi: 10.1021/acscatal.6b01138. URL https: //doi.org/10.1021/acscatal.6b01138.

[144] Stephanie Mildner, Marco Beleggia, Daniel Mierwaldt, Thomas W. Hansen, Jakob B. Wagner, Sadegh Yazdi, Takeshi Kasama, Jim Ciston, Yimei Zhu, and Christian Jooss. Environmental tem study of electron beam induced electrochemistry of pr0.64ca0.36mno3 catalysts for oxygen evolution. The Journal of Physical Chemistry C, 119(10):5301-5310, 2015. doi: 10.1021/jp511628c. URL https://doi.org/10. 1021/jp511628c.

[145] Majid Ebrahimizadeh Abrishami, Marcel Risch, Julius Scholz, Vladimir Roddatis, Norbert Osterthun, and Christian Jooss. Oxygen evolution at manganite perovskite ruddlesden-popper type particles: Trends of activity on structure, valence and covalence. Materials, 9(11), 2016. ISSN 1996-1944. doi: 10.3390/ma9110921. URL http://www.mdpi.com/1996-1944/9/11/921. 
[146] Julius Scholz, Marcel Risch, Kelsey A. Stoerzinger, Garlef Wartner, Yang Shao-Horn, and Christian Jooss. Rotating ringâĂŞdisk electrode study of oxygen evolution at a perovskite surface: Correlating activity to manganese concentration. The Journal of Physical Chemistry C, 120(49):27746-27756, 2016. doi: 10.1021/acs.jpcc.6b07654. URL https://doi.org/10.1021/acs.jpcc.6b07654.

[147] Julius Scholz, Marcel Risch, Garlef Wartner, Christoph Luderer, Vladimir Roddatis, and Christian Jooss. Tailoring the oxygen evolution activity and stability using defect chemistry. Catalysts, 7(5), 2017. ISSN 2073-4344. doi: 10.3390/catal7050139. URL http://www.mdpi.com/2073-4344/7/5/139.

[148] Daniel Mierwaldt, Vladimir Roddatis, Marcel Risch, Julius Scholz, Janis Geppert, Majid Ebrahimizadeh Abrishami, and Christian Jooss. Environmental tem investigation of electrochemical stability of perovskite and ruddlesdenâĂŞpopper type manganite oxygen evolution catalysts. Advanced Sustainable Systems, 1(12):1700109. doi: 10.1002/adsu.201700109. URL https://onlinelibrary.wiley.com/doi/abs/ 10.1002/adsu. 201700109.

[149] A. Grimaud, W. T. Hong, Y. Shao-Horn, and J.-M. Tarascon. Anionic redox processes for electrochemical devices. Nature Materials, 15:121, 2016. URL https: //doi.org/10.1038/nmat4551.

[150] Ulrike Diebold. The surface science of titanium dioxide. Surf. sci. rep., 48(5):53-229, 2003. doi: http://dx.doi.org/10.1016/S0167-5729(02)00100-0. URL http://www . sciencedirect.com/science/article/pii/S0167572902001000.

[151] Ulrike Diebold, Shao-Chun Li, and Michael Schmid. Oxide surface science. Annu. Rev. Phys. Chem., 61(1):129, 2010.

[152] S. J. Tauster, S. C. Fung, R. T. K. Baker, and J. A. Horsley. Strong interactions in supported-metal catalysts. Science, 211(4487):1121-1125, 1981. ISSN 0036-8075. doi: 10.1126/science.211.4487.1121. URL http://science.sciencemag.org/content/ $211 / 4487 / 1121$

[153] Ulf Roland, Thomas Braunschweig, and Frank Roessner. On the nature of spilt-over hydrogen. J. Mol. Catal. A: Chem., 127(1):61-84, 1997. doi: http://dx.doi.org/ 10.1016/S1381-1169(97)00110-6. URL http://www.sciencedirect.com/science/ article/pii/S1381116997001106.

[154] Jeong Young Park, L. Robert Baker, and Gabor A. Somorjai. Role of hot electrons and metalâĂSoxide interfaces in surface chemistry and catalytic reactions. Chem. Rev., 115(8):2781-2817, 2015. doi: 10.1021/cr400311p. URL http://dx.doi.org/ 10.1021/cr400311p. PMID: 25791926. 
[155] Meng Ni, Michael K.H. Leung, Dennis Y.C. Leung, and K. Sumathy. A review and recent developments in photocatalytic water-splitting using tio ${ }_{2}$ for hydrogen production. Renewable Sustainable Energy Rev., 11(3):401-425, 2007. ISSN 1364-0321. doi: http://dx.doi.org/10.1016/j.rser.2005.01.009. URL http://www . sciencedirect.com/science/article/pii/S1364032105000420.

[156] Rainer Waser, Regina Dittmann, Georgi Staikov, and Kristof Szot. Redox-based resistive switching memories âĂŞ nanoionic mechanisms, prospects, and challenges. Adv. Mater., 21(25-26):2632-2663, 2009. ISSN 1521-4095. doi: 10.1002/adma. 200900375. URL http://dx.doi.org/10.1002/adma.200900375.

[157] Yi Ma, Xiuli Wang, Yushuai Jia, Xiaobo Chen, Hongxian Han, and Can Li. Titanium dioxide-based nanomaterials for photocatalytic fuel generations. Chem. Rev., 114(19):9987-10043, 2014. doi: 10.1021/cr500008u. URL http://dx.doi.org/10. 1021/cr500008u. PMID: 25098384.

[158] Michael Bowker, Peter Stone, Roger Bennett, and Neil Perkins. \{CO $\}$ adsorption on a pd/tio2(1 1 0) model catalyst. Surf. sci., 497(1âĂŞ3):155 - 165, 2002. ISSN 0039-6028. doi: http://dx.doi.org/10.1016/S0039-6028(01)01640-5. URL http:// www.sciencedirect.com/science/article/pii/S0039602801016405.

[159] Jacinto Sá, Johannes Bernardi, and James A. Anderson. Imaging of low temperature induced smsi on $\mathrm{pd} / \mathrm{tio}_{2}$ catalysts. Catal. lett., 114(1):91-95, 2007. ISSN 1572-879X. doi: 10.1007/s10562-007-9049-1. URL http://dx.doi.org/10.1007/ s10562-007-9049-1.

[160] Jingyue Jimmy Liu. Advanced electron microscopy of metal-support interactions in supported metal catalysts. ChemCatChem, 3(6):934-948, 2011. doi: 10.1002/cctc. 201100090. URL http://dx.doi.org/10.1002/cctc. 201100090.

[161] Xiaobo Chen, Lei Liu, and Fuqiang Huang. Black titanium dioxide (tio2) nanomaterials. Chemical Society Reviews, 44(7):1861-1885, 2015. doi: 10.1039/C4CS00330F. URL http://dx.doi.org/10.1039/C4CS00330F.

[162] Oluwafunmilola Ola and M.Mercedes Maroto-Valer. Review of material design and reactor engineering on $\mathrm{tio}_{2}$ photocatalysis for $\mathrm{co}_{2}$ reduction. J. Photochem. Photobiol., C: Photochem. Rev., 24:16-42, 2015. ISSN 1389-5567. doi: http://dx.doi. org/10.1016/j.jphotochemrev.2015.06.001. URL http://www.sciencedirect.com/ science/article/pii/S1389556715000271.

[163] A. Fujishima and K. Honda. Electrochemical photolysis of water at a semiconductor eleectrode. Nature, 238:37, 1972.

[164] M. Cerchez, H. Langer, M. El Achhab, T. Heinzel, D. Ostermann, H. LÃijder, and J. Degenhardt. Dynamics of hydrogen sensing with pt/tio2 schottky diodes. Appl. 
Phys. Lett., 103(3):033522, 2013. doi: 10.1063/1.4816265. URL http://dx.doi. org/10.1063/1.4816265.

[165] Michael R. Hoffmann, Scot T. Martin, Wonyong. Choi, and Detlef W. Bahnemann. Environmental applications of semiconductor photocatalysis. Chem. Rev., 95(1):69-96, 1995. doi: 10.1021/cr00033a004. URL http://dx.doi.org/10.1021/ cr00033a004.

[166] Xiaobo Chen, Shaohua Shen, Liejin Guo, and Samuel S. Mao. Semiconductor-based photocatalytic hydrogen generation. Chem. Rev., 110(11):6503-6570, 2010. doi: 10. 1021/cr1001645. URL http://dx.doi.org/10.1021/cr1001645. PMID: 21062099.

[167] R. Asahi, T. Morikawa, T. Ohwaki, K. Aoki, and Y. Taga. Visible-light photocatalysis in nitrogen-doped titanium oxides. Science, 293(5528):269-271, 2001. doi: 10.1126/ science.1061051. URL http://science.sciencemag.org/content/293/5528/269.

[168] Xiaobo Chen, Lei Liu, Peter Y. Yu, and Samuel S. Mao. Increasing solar absorption for photocatalysis with black hydrogenated titanium dioxide nanocrystals. Science, 331(6018):746-750, 2011. ISSN 0036-8075. doi: 10.1126/science.1200448. URL http: //science.sciencemag.org/content/331/6018/746.

[169] Wonyong Choi, Andreas Termin, and Michael R Hoffmann. The role of metal ion dopants in quantum-sized tio2: Correlation between photoreactivity and charge carrier recombination dynamics. J. Phys. Chem., 98(51):13669-13679, 1994.

[170] T. Umebayashi, T. Yamaki, H. Itoh, and K. Asai. Band gap narrowing of titanium dioxide by sulfur doping. Appl. Phys. Lett., 81(3):454-456, 2002. doi: 10.1063/1. 1493647. URL http://dx.doi.org/10.1063/1.1493647.

[171] Jong Hyeok Park, Sungwook Kim, and Allen J. Bard. Novel carbon-doped tio2 nanotube arrays with high aspect ratios for efficient solar water splitting. Nano Lett., 6(1):24-28, 2006. doi: 10.1021/nl051807y. URL http://dx.doi.org/10. 1021/nl051807y. PMID: 16402781.

[172] Fan Zuo, Le Wang, Tao Wu, Zhenyu Zhang, Dan Borchardt, and Pingyun Feng. Self-doped ti3 + enhanced photocatalyst for hydrogen production under visible light. J. Am. Chem. Soc., 132(34):11856-11857, 2010. doi: 10.1021/ja103843d. URL http: //dx.doi.org/10.1021/ja103843d. PMID: 20687606.

[173] Xiaobo Chen and Clemens Burda. The electronic origin of the visible-light absorption properties of c-, n- and s-doped tio2 nanomaterials. J. Am. Chem. Soc., 130(15):50185019, 2008. doi: 10.1021/ja711023z. URL http://dx.doi.org/10.1021/ja711023z. PMID: 18361492.

[174] Ulrich Aschauer and Annabella Selloni. Hydrogen interaction with the anatase tio2(101) surface. Phys. Chem. Chem. Phys., 14:16595-16602, 2012. doi: 10.1039/ C2CP42288C. URL http://dx.doi.org/10.1039/C2CP42288C. 
[175] M. Sotoudeh, S. J. Hashemifar, M. Abbasnejad, and M. R. Mohammadizadeh. Abinitio study of hydrogen doping and oxygen vacancy at anatase tio2 surface. AIP Adv., 4(2):027129, 2014. doi: 10.1063/1.4866982. URL http://dx.doi.org/10. $\overline{1063} / 1.4866982$.

[176] M. Sotoudeh, M. Abbasnejad, and M. R. Mohammadizadeh. First principles study of hydrogen doping in anatase tio $_{2}$. Eur. Phys. J. Appl. Phys., 67(3):30401, 2014. doi: 10.1051/epjap/2014130582. URL https://doi.org/10.1051/epjap/2014130582.

[177] L. B. Mo, Y Wang, Yang Bai, Q. Y. Xiang, Qun Li, W. Q. Yao, J. O. Wang, Kurash Ibrahim, H. H. Wang, C. H. Wan, and J. L. Cao. Hydrogen impurity defects in rutile tio $_{2}$. Sci. Rep., 5:17634, 2015. doi: 10.1038/srep17634. URL http://dx.doi.org/ $10.1038 /$ srep 17634 .

[178] Madhavan Ramamoorthy, R. D. King-Smith, and David Vanderbilt. Defects on tio 2 (110) surfaces. Phys. Rev. B, 49:7709-7715, Mar 1994. doi: 10.1103/PhysRevB.49. 7709. URL http://link.aps.org/doi/10.1103/PhysRevB.49.7709.

[179] Eunae Cho, Seungwu Han, Hyo-Shin Ahn, Kwang-Ryeol Lee, Seong Keun Kim, and Cheol Seong Hwang. First-principles study of point defects in rutile $\mathrm{Tio}_{2-x}$. Phys. Rev. B, 73:193202, May 2006. doi: 10.1103/PhysRevB.73.193202. URL http: //link.aps.org/doi/10.1103/PhysRevB.73.193202.

[180] James M. Sullivan and Steven C. Erwin. Theory of dopants and defects in co-doped tio $_{2}$ anatase. Phys. Rev. B, 67:144415, Apr 2003. doi: 10.1103/PhysRevB.67.144415. URL http://link.aps.org/doi/10.1103/PhysRevB.67.144415.

[181] Sutassana Na-Phattalung, M. F. Smith, Kwiseon Kim, Mao-Hua Du, Su-Huai Wei, S. B. Zhang, and Sukit Limpijumnong. First-principles study of native defects in anatase $\mathrm{TiO}_{2}$. Phys. Rev. B, 73:125205, Mar 2006. doi: 10.1103/PhysRevB.73.125205. URL http://link.aps.org/doi/10.1103/PhysRevB.73.125205.

[182] Paula Mori-Sánchez, Aron J. Cohen, and Weitao Yang. Localization and delocalization errors in density functional theory and implications for band-gap prediction. Phys. Rev. Lett., 100:146401, Apr 2008. doi: 10.1103/PhysRevLett.100.146401. URL http://link.aps.org/doi/10.1103/PhysRevLett.100.146401.

[183] Gianfranco Pacchioni. Modeling doped and defective oxides in catalysis with density functional theory methods: Room for improvements. J. Chem. Phys., 128(18):182505, 2008. doi: 10.1063/1.2819245. URL http://dx.doi.org/10.1063/1.2819245.

[184] G. Mattioli, F. Filippone, P. Alippi, and A. Amore Bonapasta. Ab initio study of the electronic states induced by oxygen vacancies in rutile and anatase $\mathrm{TiO}_{2}$. Phys. Rev. B, 78:241201, Dec 2008. doi: 10.1103/PhysRevB.78.241201. URL http://link. aps.org/doi/10.1103/PhysRevB.78.241201. 
[185] Benjamin J. Morgan and Graeme W. Watson. Polaronic trapping of electrons and holes by native defects in anatase tio ${ }_{2}$. Phys. Rev. B, 80:233102, Dec 2009. doi: 10. 1103/PhysRevB.80.233102. URL http://link.aps.org/doi/10.1103/PhysRevB. 80.233102.

[186] Cristiana Di Valentin, Gianfranco Pacchioni, and Annabella Selloni. Reduced and n-type doped tio2: Nature of ti3 + species. J. Phys. Chem. C, 113(48):20543-20552, 2009. doi: 10.1021/jp9061797. URL http: //dx.doi.org/10.1021/jp9061797.

[187] A. Janotti, J. B. Varley, P. Rinke, N. Umezawa, G. Kresse, and C. G. Van de Walle. Hybrid functional studies of the oxygen vacancy in tio ${ }_{2}$. Phys. Rev. B, 81:085212, Feb 2010. doi: 10.1103/PhysRevB.81.085212. URL http://link.aps.org/doi/10. 1103/PhysRevB . 81.085212.

[188] Hsin-Yi Lee, Stewart J. Clark, and John Robertson. Calculation of point defects in rutile $\mathrm{tio}_{2}$ by the screened-exchange hybrid functional. Phys. Rev. B, 86:075209, Aug 2012. doi: 10.1103/PhysRevB.86.075209. URL http://link.aps.org/doi/ 10.1103/PhysRevB.86.075209.

[189] Shang-Di Mo and W. Y. Ching. Electronic and optical properties of three phases of titanium dioxide: Rutile, anatase, and brookite. Phys. Rev. B, 51:13023-13032, May 1995. doi: 10.1103/PhysRevB.51.13023. URL http://link.aps.org/doi/10. 1103/PhysRevB.51.13023.

[190] R Brydson, H Sauer, W Engel, J M Thomass, E Zeitler, N Kosugi, and H Kuroda. Electron energy loss and x-ray absorption spectroscopy of rutile and anatase: a test of structural sensitivity. Journal of Physics: Condensed Matter, 1(4):797, 1989. URL http://stacks.iop.org/0953-8984/1/i=4/a=012.

[191] E. Stoyanov, F. Langenhorst, and G. Steinle-Neumann. The effect of valence state and site geometry on ti 13,2 and o k electron energy-loss spectra of tixoy phases. American Mineralogist, 92(4):577-586, 2007. ISSN 0003-004X. doi: 10.2138/am. 2007.2344. URL http://ammin.geoscienceworld.org/content/92/4/577.

[192] Marian David Bongers. In situ studies on palladium/rutile titanium dioxide exposed to low pressure hydrogen gas environments. PhD thesis, Fakultät für Physik (inkl. GAUSS), Georg-August-Universität Göttingen, 2018.

[193] Ali Moballegh and Elizabeth C. Dickey. Electric-field-induced point defect redistribution in single-crystal $\mathrm{tio}_{2-x}$ and effects on electrical transport. Acta Materialia, 86:352-360, 2015. doi: 10.1016/j.actamat.2014.11.032. URL http: //www.sciencedirect.com/science/article/pii/S1359645414008854.

[194] J. Pascual, J. Camassel, and H. Mathieu. Fine structure in the intrinsic absorption edge of $\mathrm{tio}_{2}$. Phys. Rev. B, 18:5606-5614, Nov 1978. doi: 10.1103/PhysRevB.18.5606. URL http://link.aps.org/doi/10.1103/PhysRevB.18.5606. 
[195] Don T. Cromer and K. Herrington. The Structures of Anatase and Rutile. Journal of the American Chemical Society, 77(18):4708-4709, 1955. doi: 10.1021/ja01623a004. URL https://pubs.acs.org/doi/abs/10.1021/ja01623a004.

[196] L. D. Finkelstein, E. Z. Kurmaev, M. A. Korotin, A. Moewes, B. Schneider, S. M. Butorin, J-H. Guo, J. Nordgren, D. Hartmann, M. Neumann, and D. L. Ederer. Band approach to the excitation-energy dependence of x-ray fluorescence of $\mathrm{tio}_{2}$. Phys. Rev. B, 60:2212-2217, Jul 1999. doi: 10.1103/PhysRevB.60.2212. URL http: //link.aps.org/doi/10.1103/PhysRevB.60.2212.

[197] Peter B. Wells and Anthony G. Wilkinson. Platinum group metals as heterogeneous enantioselective catalysts. Top. Catal., 5(1-4):39-50, 1998. ISSN 1572-9028. doi: 10.1023/A:1019197802585. URL http://dx.doi.org/10.1023/A:1019197802585.

[198] Detre Teschner, János Borsodi, Attila Wootsch, Zsolt Révay, Michael Hävecker, Axel Knop-Gericke, S. David Jackson, and Robert Schlögl. The roles of subsurface carbon and hydrogen in palladium-catalyzed alkyne hydrogenation. Science, 320(5872):8689, 2008. ISSN 0036-8075. doi: 10.1126/science.1155200. URL http://science. sciencemag.org/content/320/5872/86.

[199] Björn Brandt, Wiebke Ludwig, Jan-Henrik Fischer, Jörg Libuda, Francisco Zaera, and Swetlana Schauermann. Conversion of cis- and trans-2-butene with deuterium on a pd/fe3o4 model catalyst. J. Catal., 265(2):191-198, 2009. ISSN 0021-9517. doi: http://dx.doi.org/10.1016/j.jcat.2009.05.005. URL http://www . sciencedirect.com/science/article/pii/S0021951709001511.

[200] G Sandrock, S Suda, L Schlapbach, and L Schlapbach. Hydrogen in intermetallic compounds ii. Topics in Applied Physics, 67:197, 1992.

[201] E. Wicke, H. Brodowsky, and H. Züchner. Hydrogen in palladium and palladium alloys, pages 73-155. Springer Berlin Heidelberg, Berlin, Heidelberg, 1978. ISBN 978-3-540-35801-5. doi: 10.1007/3-540-08883-0_19. URL https://doi.org/10. 1007/3-540-08883-0_19.

[202] F. D. Manchester, A. San-Martin, and J. M. Pitre. The h-pd (hydrogen-palladium) system. J. Phase Equilib., 15(1):62-83, 1994. ISSN 1054-9714. doi: 10.1007/ BF02667685. URL http://dx.doi.org/10.1007/BF02667685.

[203] Adolf Sieverts. The absorption of gases by metals. Zeitschrift für Metallkunde, 21: 37-46, 1929.

[204] T B Flanagan and W A Oates. The palladium-hydrogen system. Annual Review of Materials Science, 21(1):269-304, 1991. doi: 10.1146/annurev.ms.21.080191.001413. URL https://doi .org/10.1146/annurev.ms.21.080191.001413. 
[205] A. Pundt and R. Kirchheim. Hydrogen in metals: Microstructural aspects. Annual Review of Materials Research, 36(1):555-608, 2006. doi: 10.1146/annurev.matsci. $\overline{36.090804 .094451 . \text { URL https }}$ ///doi .org/10.1146/annurev.matsci. 36.090804. 094451.

[206] Reiner Kirchheim. Reducing grain boundary, dislocation line and vacancy formation energies by solute segregation. i. theoretical background. Acta Materialia, 55(15):5129-5138, 2007. ISSN 1359-6454. doi: http://dx.doi.org/10.1016/j. actamat.2007.05.047. URL http://www.sciencedirect.com/science/article/ $\mathrm{pii} / \mathrm{S} 1359645407003655$.

[207] Reiner Kirchheim. Reducing grain boundary, dislocation line and vacancy formation energies by solute segregation: Ii. experimental evidence and consequences. Acta materialia, 55(15):5139-5148, 2007. ISSN 1359-6454. doi: http://dx.doi.org/10.1016/ j.actamat.2007.05.033. URL http://www.sciencedirect.com/science/article/ pii/S1359645407003667.

[208] Stefan Wagner and Astrid Pundt. Quasi-thermodynamic model on hydride formation in palladium-hydrogen thin films: Impact of elastic and microstructural constraints. Int. J. Hydrog. Energy, 41(4):2727-2738, 2016. ISSN 0360-3199. doi: http:// dx.doi.org/10.1016/j.ijhydene.2015.11.063. URL http://www.sciencedirect.com/ science/article/pii/S0360319915310272.

[209] Stefan Wagner, Thilo Kramer, Helmut Uchida, Patrik Dobron, Jakub Cizek, and Astrid Pundt. Mechanical stress and stress release channels in 10âĂŞ350 nm palladium hydrogen thin films with different micro-structures. Acta Materialia, 114:116 - 125, 2016. ISSN 1359-6454. doi: http://dx.doi.org/10.1016/j. actamat.2016.05.023. URL http://www.sciencedirect.com/science/article/ $\mathrm{pii} / \mathrm{S} 1359645416303615$.

[210] Helmut Mehrer. Diffusion in solids: fundamentals, methods, materials, diffusion-controlled processes, volume 155. Springer Science \& Business Media, 2007.

[211] N. Yamamoto, S. Tonomura, T. Matsuoka, and H. Tsubomura. A study on a palladium-titanium oxide schottky diode as a detector for gaseous components. Surf. Sci., 92(2-3):400-406, 1980. ISSN 0039-6028. doi: http://dx.doi.org/10.1016/ 0039-6028(80)90212-5. URL http://www.sciencedirect.com/science/article/ pii/0039602880902125.

[212] M. Strungaru, M. Cerchez, S. Herbertz, T. Heinzel, M. El Achhab, and K. Schierbaum. Interdependence of electroforming and hydrogen incorporation in nanoporous titanium dioxide. Appl. Phys. Lett., 106(14):143109, 2015. doi: 10.1063/1.4917034. URL http://dx.doi.org/10.1063/1.4917034.

[213] O. Jepson and O.K. Anderson. The electronic structure of h.c.p. ytterbium. Solid State Communications, 9(20):1763 - 1767, 1971. ISSN 0038-1098. doi: https: 
//doi.org/10.1016/0038-1098(71)90313-9. URL http://www.sciencedirect.com/ science/article/pii/0038109871903139.

[214] G. Lehmann and M. Taut. On the numerical calculation of the density of states and related properties. physica status solidi (b), 54(2):469-477. doi: 10.1002/pssb. 2220540211. URL https://onlinelibrary.wiley.com/doi/abs/10.1002/pssb. 2220540211.

[215] Peter E. Blöchl, O. Jepsen, and O. K. Andersen. Improved tetrahedron method for brillouin-zone integrations. Phys. Rev. B, 49:16223-16233, Jun 1994. doi: 10. 1103/PhysRevB.49.16223. URL http://link.aps.org/doi/10.1103/PhysRevB. 49. 16223.

[216] Don T. Cromer and K. Herrington. The structures of anatase and rutile. J. Am. Chem. Soc., 77(18):4708-4709, 1955. doi: 10.1021/ja01623a004. URL http://dx. doi.org/10.1021/ja01623a004.

[217] W. F. Kuhs and M. S. Lehmann. The structure of the ice ih by neutron diffraction. J. Phys. Chem., 87(21):4312-4313, 1983. doi: 10.1021/j100244a063. URL http: //dx.doi.org/10.1021/j100244a063.

[218] Victor E. Henrich, G. Dresselhaus, and H. J. Zeiger. Observation of two-dimensional phases associated with defect states on the surface of tio 2 . Phys. Rev. Lett., 36: 1335-1339, May 1976. doi: 10.1103/PhysRevLett.36.1335. URL http://link.aps. org/doi/10.1103/PhysRevLett.36.1335.

[219] P. E. Blöchl. The LDA+DMFT approach to strongly correlated materials, volume 1 of Modelling and Simulation, chapter Theory and Practice of Density functional theory, pages 2.1-2.44. Forschungszentrum Jülich GmbH, Institute for Advanced Simulations, 2011.

[220] R. McWeeny. Some recent advances in density matrix theory. Rev. Mod. Phys., 32: 335-369, Apr 1960. doi: 10.1103/RevModPhys.32.335. URL https://link.aps. org/doi/10.1103/RevModPhys.32.335.

[221] Peter J. Schultz and K. G. Lynn. Interaction of positron beams with surfaces, thin films, and interfaces. Rev. Mod. Phys., 60:701-779, Jul 1988. doi: 10.1103/ RevModPhys.60.701. URL http://link.aps.org/doi/10.1103/RevModPhys.60. 701.

[222] Wolfgang Anwand, Gerhard Brauer, Maik Butterling, Hans Rainer Kissener, and Andreas Wagner. Design and construction of a slow positron beam for solid and surface investigations. Defect and Diffusion Forum, 331:25-40, 2012. doi: 10.4028/ www.scientific.net/DDF.331.25. URL https://www.scientific.net/DDF.331.25. 
[223] Reinhard Krause-Rehberg and Hartmut S. Leipner. Positron Annihilation in Semiconductors: Defect Studies. Springer-Verlag Berlin Heidelberg, 1999. ISBN 978-3-540-64371-5.

[224] M. W. Jr. CHASE. Nist-janaf thermochemical tables fourth edition. J. Phys. Chem. Ref. Data, Monograph, 9, 1998. URL https://ci.nii.ac.jp/naid/ $\overline{10026079537 /}$ en/.

[225] T. Shimanouchi. Tables of molecular vibrational frequencies. consolidated volume ii. Journal of Physical and Chemical Reference Data, 6(3):993-1102, 1977 . doi:

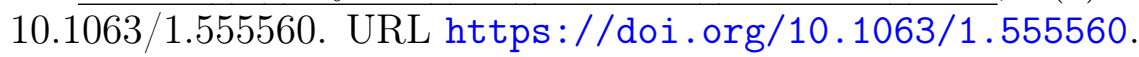

[226] Carl W. David. The tensor of the moment of inertia. Chemistry Education Materials., 21, 2006. URL http://digitalcommons.uconn.edu/chem_educ/21. 


\section{Acknowledgements}

I would like to express my deepest gratitude to many colleagues at the Institute for Theoretical Physics, Institute for Material Physics, friends, who have been supporting the completion of the present thesis.

First of all, I would like to name my thesis adviser Prof. Peter Blöchl for his scientific discussions, pronounced commitment, responsibility, guidance, compassion and support in my work. I am very thankful for the inspirational discussions, patience and reliability. I also thank Prof. Christian Jooss for his encouraging and interesting discussions from the experimental point of view.

Dr. Marian Bongers and Prof. Astrid Pundt provided substantial support and enlightened me about defects in $\mathrm{TiO}_{2}$ by comprehensible reports and discussions about physics and chemistry. I am very grateful for the accompanying and endless efforts and giving me new insights into the relation between semiconductor physics and the electronic band structure of metal-rutile junction. I greatly appreciate the conversations with PD Dr. Martin Wenderoth who enriched my understanding of the $p$-n junction in semiconductors. I would like to thank Dr. Thorsten Stolper for his fruitful discussions from the chemistry point of view. Moreover, I would like to express my thanks to the students of my working group for contributing to a comfortable working atmosphere with humor and exciting philosophical conversations. In particular, I want to mention Robert Schade, Sangeeta Rajpurohit, Debanjan Basu, Ingolf Heinrich Harden, Philipp Seichter and Michael ten Brink for numerous exciting hours at the group. I appreciate the help of Sigrid Frömmel to overcome German bureaucracy and paperwork. I would like to thank Axel Ehrich for his support to all of the technical issues.

I am so thankful to my wife Salimeh for her love and support and reminding me of the life beside the doctoral studies.

Last but not least, I am very grateful to my parents for their help and support.

Mohsen Sotoudeh 